Portland State University

PDXScholar

$1-1-1980$

\title{
Selective reflection of light at a solid-gas interface and its application
}

Fumihide Takeda

Portland State University

Follow this and additional works at: https://pdxscholar.library.pdx.edu/open_access_etds

\section{Let us know how access to this document benefits you.}

\section{Recommended Citation}

Takeda, Fumihide, "Selective reflection of light at a solid-gas interface and its application" (1980). Dissertations and Theses. Paper 838.

https://doi.org/10.15760/etd.838

This Dissertation is brought to you for free and open access. It has been accepted for inclusion in Dissertations and Theses by an authorized administrator of PDXScholar. Please contact us if we can make this document more accessible: pdxscholar@pdx.edu. 


\title{
SELECTIVE REFIECTION OF IIGHT AT A SOLID-GAS INTERFACE AND ITS APPLICATION
}

\section{by}

FUNIHIDE TAKEDA

A dissertation submitted in partial fulfillment of the requirements for the degree of

\author{
DOCTOR OF PHILOSOPHY \\ in \\ ENVIRONMENTAI SCIENCE \\ AND RESOURCES - PHYSICS
}

Portland State University

1980

(C) Fumihide Takeda 1980 
TO THE OFFICE OF GRADUATE STUDIES AND RESEARCH:

The members of the Committee approve the dissertation of Fumihide Takeda presented May 20, 1980.
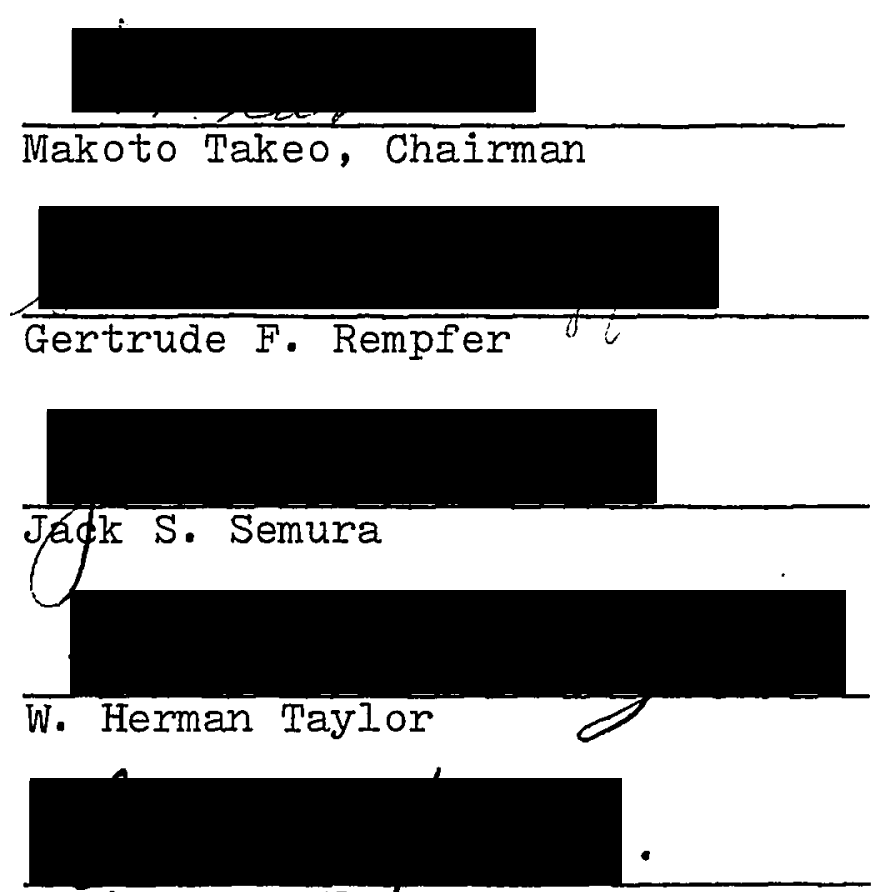

David W. McClure

\section{APPROVED:}

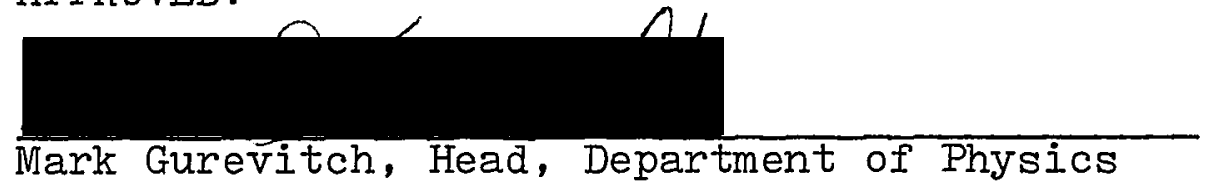

Stanley Rauch, Lean of Graduate Studies and Research 
AN ABSTRACT OF THE DISSERTATION OF Fumihide Takeda for the Doctor of Philosophy in Environmental Sciences and Resources - Physics presented April 29, 1980.

Title: Selective Reflection of light At A Solid-Gas Interface And Its Application.

APPROVED BY MEMBERS OF THE DISSERTATION CONMITTEE:

Makoto Takeo, Chairman
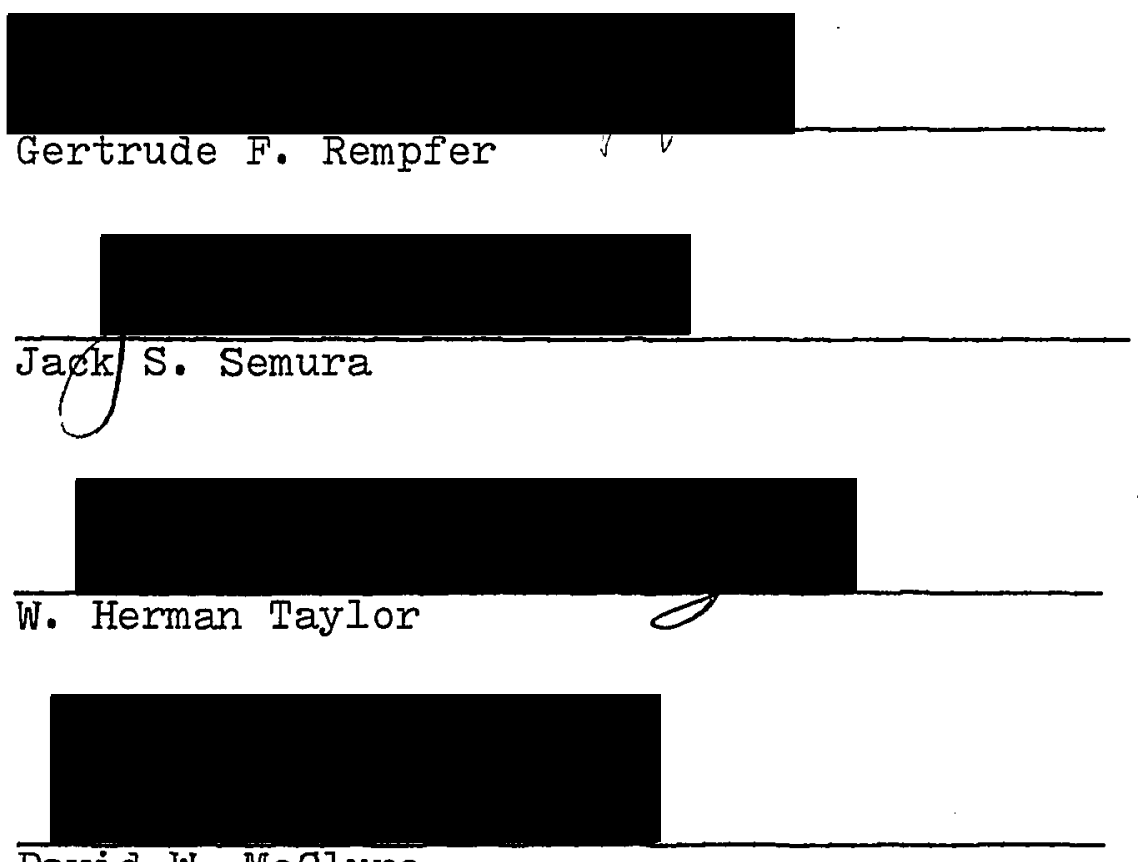

David W. MeClure 
In order to study the detailed spectral intensity distribution of light reflected from a solid-gas interface, the extinction theorem in optics is extended to include an absorbing medium and the thermal motion of the gas molecules near the interface. The theoretical spectral intensity distribution in the region of anomalous dispersion is found to be strongly modified compared to that predicted by existing theory.

An important consequence of this theory in the line shapes of the reflected light is the possibility of using recently developed saturation spectroscopic techniques to study atoms and molecules near surfaces.

In order to investigate the feasibility of these new techniques for obtaining solid-gas molecule interaction potentials, models of solid-gas interfaces were studied with and without interactions of the type $1 / z^{p}(p=2,3,4)$, where $\mathrm{z}$ is the distance between gas molecules and solid surface. A marked difference in the line shapes of the reflected light among the possible interactions suggests that the forms of interaction at the interface can be measured using known techniques.

Furthermore, the possibility of measuring the flow of gas near walls where currently available laser-Doppler anemometers can not spatially reach is investigated. It is shown that the shift and width of the numerically calculated line shape of the reflected light in our model flow is directly related to the mean and the fluctuating velocity fields respectively. 


\section{ACKNOWIEDGEMENTS}

To the memory of my late grandmother, Hamayo Kasasaki, I dedicate my dissertation.

I wish to thank my old professor, Dr. Takeshi Satoh of the Japanese National Defense Academy, for encouraging me to further my education at P. S. U..

Also I wish to express my great appreciation to Dr. Makoto Takeo for giving me this very interesting subject and for his support and advice. His advice was based upon not only his solid background in Mathematical Physics, but also his extraordinary insight in grasping physical phenomena.

I appreciate the encouragement and support given to me by Dr. Mark Gurevitch, Dr. Gertrude Rempfer, and remaining professors and staff within the Physics Department.

I also wish to thank my friends, in particular, Dorothy Sackett, Jeff Fossum, Greg Ordway and his parents for sharing enjoyable times.

Finally I wish to thank the following friends, Dave Cress, Dennis Munsterman, Dorothy Sackett, and Kostas Antonopoulos for their time and efforts in completion of this dissertation on my behalf. 
TABLE OF CONTENTS

PAGE

ACKNOWLEDGNENTS....................... i. ii

LIST OF FIGURES...................... vi

CHAPTER

I INTRODUCTION................... 1

II SOLUTIONS OF MAXWELI'S WAVE EQUATIONS

AND THE EXTINCTION THEOREM;........

8

Partially Non-Local (In Space)

Representations

1. Introduction................. 8

2. Material Bounded By A Vacuum...... 9

3. Material Bounded By Another Material

Medium..................... 20

4. A Simple Application of The Extinction Theorem And The Lorentz-Lorenz

Formula...................

5. Summary of The Generalized Extinction Theorem...................

II THE EXTINCTION THEOREM IN ANOMALOUS

DISPERSIDN WITH PIANE BOUNDARIES . . . . .

30

1. Introduction................ 30

2. Fully Non-Local Representations (In Space And Time) Of Formal Solutions For Maxwell's Wave Equations......

3. A New Derivation Df Snell's Law And Fresnel's Formula..............

4. A Derivation of Fresnel's Formula For The Case of A Sharp Boundary.. 
IV SELECTIVE REFLECTION OF IIGHT FROM A SOLID-GAS INTERFACE................ 52

1. Introduction.................... 52

2. The Macroscopic Polarization Field Within The Penetration Layer........ 54

3. The Iine Shape of The Reflected Iight. 67

V APPIICATION OF SATURATION SPECTROSCDPY TO SURFACE INVESTIGATION.............. 71

I. Introduction................. 71

2. Reflection At A Solid-Gas Interface Without Interactions............ 72

3. Reflection At A Solid-Gas Interface with Interactions............... 78

VI APPLICATION OF TUNABLE IASER TO GAS FLOW MEASUREMENTS NEAR A WAIL............. 86

I. Introduction................... 86

2. Response of The Weak Beam To Gas Flow. 87

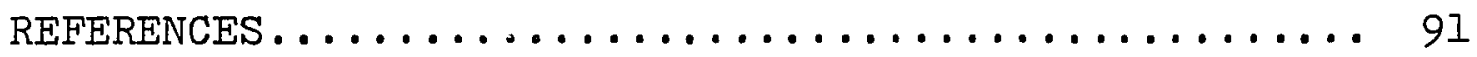
APPENDIX A

Computer Program And Results

For Chapter IV.................... 93

APPENDIX B

Computer Program And Results

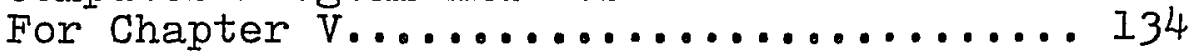

APPENDIX C

Computer Program And Results

For Chapter VI.................... 172 


\section{IIST OF FIGURES}

FIGURE

PAGE

2.1 A Material Bounded By A Medium $M_{0} \ldots . . . .10$

4.1 A Measured Reflectivity Of A Glass-

Na Vapor Interface ............. 54

5.1 A Solid-Gas Interface ............... 74

5.2 Reflectivity In Saturation Spectro-

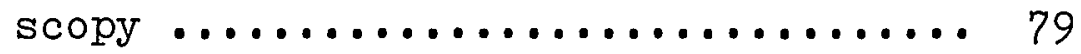

5.3 A Thin Metal Layer (Deposited On A

GIass) - Gas Interface ........... 8 I

5.4 Intensity Distributions Among The

Possible Interactions ........... 85 


\section{CHAPTER I}

\section{INTRODUCTION}

In order to understand surface phenomena in relation to lubrication, corrosion, catalysis, emissive and adsorptive properties, and adhesion, it is appropriate to first study the static characteristics in terms of the chemical identity of atoms present on the surface, their geometrical arrangement, and their electronic states. Experimental probes used to investigate surfaces are photons, electrons, ions, atoms, molecules, external fields, and their various combinations (J. R. Schrieffer, et. al., 1975). Although the methods developed for using these probes are ingenious, they have limitations in practice due to their technical and physical sensitivity to the surface involved. The response of the surface to an externally applied probe has the following general energy resolutions: They are of the

order of $1-10^{-1} \mathrm{eV}$ for LEED, $10^{-1}-10^{-2} \mathrm{eV}$ for ion beam spectroscopy (atomic or molecular beam spectroscopy), and $0.2-0.3 \mathrm{eV}$ for photoemission spectroscopy. For detailed study of surface properties, however, the above energy resolutions are probably inadequate for resolving the energy change in electronic states in an adsorbed atom. In fact, adsorption on a surface can occur with binding energy of the order of $10^{-2} \mathrm{eV}$ (physisorption). 
Now consider a solid in contact with gas. For the gas (atoms or molecules), the detailed structure of the characteristic frequency is in general strongly modified by Doppler broadening. However, J. I. Cojan (1954) and J. P. Woerdman and M. F. H. Schuurmans (1974) observed an increased resolution (due to a partial absence of Doppler broadening) in light reflected normal to the solid-gas interface when its frequency was in the anomalous dispersion region. As a consequence, the dynamic response of the atom's own internal state to the surface may be detected through the anomalous dispersion of the reflected light. This particular reflection of light has been known as "selective reflection of light" which was first investigated many years ago by $R$. W. Wood (See the text of R. W. Wood, 1934). However, a satisfactory theory of the phenomenon has not been reported to date.

In this dissertation, we report on a theoretical analysis of optical reflection and refraction related to anomalous dispersion at a solid-gas interface. An important result of inis study predicts a large increase (due to near absence of Doppler broadening) of the previously observed resolution. Therefore we place emphasis on its possible application as a new surface probe for environmentally related interface phenomena, such as, a model interface between a thin metal layer deposited on glass and $\mathrm{Na}$ vapor (or any other atom or molecule).

Another possible application will be in the field of 
flow measurements near a solid surface. In our daily environment, we encounter not only natural flows (such as, wind, river and ocean currents, etc.) but also artificially created flows (such as, flows in laboratory systems, in power plants, etc.). Both types of flows are very complex and important to our environment. In an effort to improve our environmental resources or protect them from destruction, the close interplay between the flow measurements and theory has been successfully applied to the modeling of some complex flows. (See summer lecture notes of B. E. Launder, K. Hanjalić, H. A. Dwyer and F. J. Durst, 1978 and 1979.) In flow measuring techniques, the optical method has advanced with the development of Laser Doppler Anemometry (I.D.A.) systems. (See the text of F. Durst, A. Melling and J. H. Whitelaw, 1976). Until recently, technical problems have limited their use mainly to artificially created flows. However, a recent development in the mechanical flow meter (propeller type) will make possible accurate measurements of natural flows (F. Takeda and R. Takeda, 1979).

In modeling the flow of fluids in the environment, measurements of flow within the boundary layer have an essential role in predicting any complex flow. This is due to the difficulty of modeling turbulent flows on digital computers. The lack of physical information on the rapidly changing velocity field within the boundary layer causes not only a severe numerical problem, but also a substantial increase of computer storage and computing time. Unfortun- 
ately, currently available L.D.A. techniques cannot reach the region of gas flow very close to the boundary. However, this region can be explored by using reflected light under conditions of anomalous dispersion.

In order to theoretically determine the unique properties of reflected light in the region of anomalous dispersion, we must note that the penetration depth of the incident light is very often of the order of the mean free path of the gaseous atom. In such a case, we cannot use the well known continuity conditions at the interface for the solutions of the macroscopic Maxwell's wave equations. This formal method is only applicable for the bulk gas (of known optical properties), not that within the penetration depth. Even if we use the familiar "surface impedance" method to solve this optical reflection and refraction problem, the precise optical properties at the interface are still required. Thus it would be more appropriate and may sometimes be imperative to use microscopic field descriptions at the beginning, and then to appropriately average them. These averaged quantities will be the macroscopic field quantities of interest.

This approach can in principle be used for any system. However, for simplicity, let us consider the case when light enters from a vacuum into a gaseous medium. Even though we will observe only the refracted light in the medium, we will consider that the incident light enters the gaseous medium without modification of phase, amplitude, phase velocity or 
propagation direction. However, due to scattering of the incident light by the gaseous atoms, extinction of the incident light occurs everywhere in the medium. As a result, the refracted light becomes the macroscopic description of all the microscopic fields and propagates with the phase velocity of the medium. This extinction of the incident light, which has a spatially non-local nature, is known as the "Ewald-Oseen extinction theorem" in classical molecular optics. (See, for instance, the text of M. Born and E. Wolf, 1975a.) This unique relationship between the incident light and refracted light may be regarded as a spatially non-local version of the usual boundary condition used in order to obtain the unique response of the medium to the incident light. This point of view, based on classical molecular optics, has been successfully applied by E. Lalor and $E$. Wolf (1972) to derive the well known reflection and refraction law of electromagnetic waves at a geometrically sharp interface between a vacuum and a semi-infinite, linear, isotropic, homogeneous, non-absorbing dielectric. Here the words "geometrically sharp" mean that the optical properties at the interface are the same everywhere in the medium. However, when the absorption of the medium is strong, the resulting field of the light in the medium can change drastically over the mean free path of the gaseous atoms near the interface. This requires a special averaging process of the scattered field, as described in Chapter IV. A generalization of the Ewald-Oseen extinction theor- 
em based on macroscopic Maxwell's equations was initiated by J. J. Sein (1970) and has been carried out by J. deGeode and P. Muzar (1972), D. N. Pattanayak and E. Wolf (1972), and E. Wolf (1976). They considered an interface between a vacuum and a simple generalized material as a geometrically sharp one where the electromagnetic wave's properties are the same as that of the bulk material. Their results cannot be directly applied to our case because the optical properties at the interface are different from those of the bulk medium. However, using mathematical techniques similar to those used by the above investigators, we derive a formal solution of the macroscopic Maxwell's wave equations in the form of a spatially non-local representation. As a result, our formal solutions contain interface effects due to the physical boundary. Thus in Chapter II, we first formulate various formal solutions for the response of a simple generalized material, including physical surface effects, to incident radiation whose source is located in the medium enclosing the material. The medium enclosing the material is not necessarily a vacuum and may be replaced by any other material, for example, a simple dielectric. The formal solutions contain two different spatial points. One is the observation point. The other is an induced source point in the material, from which the incident radiation is scattered.

In Chapter III, we remove this restriction and derive fully non-local (in space) representations. Then we can express the spatially non-local nature of the physical 
extinction of the incident light in mathematical form. Furthermore, by using this extinction theorem as a nonlocal boundary condition, we obtain Fresnel's formulas of anomalous dispersion from the set of formal solutions obtained in Chapter II.

In Chapter IV, using the results of Chapter III, we explain the increased resolution of the reflected light in the frequency region of anomalous dispersion which was mentioned at the beginning of this chapter. We also obtain numerically calculated theoretical line shapes of the reflected light, including the case where the incident angle is larger than the critical angle.

In Chapter $V$, we use the phenomena predicted in Chapter IV and apply recently developed laser-saturation spectroscopic techniques to a model interface of a thin metal layer deposited on glass with $\mathrm{Na}$ vapor as the gas. The last chapter explores possible gas flow measurement techniques. The computer program used for numerical calculations and the results are presented in the appendices. 


\section{CHAPTER II}

\section{SOLUTIONS OF MAXWELI'S WAVE EQUATIONS AND THE EXTINCTION THEOREM \\ PARTIALIY NON-LOCAI (IN SPACE) REPRESENTATIONS}

\section{INTRODUCTION}

In order to establish the basis for the treatment of the extinction theorem, we derive formal solutions of the macroscopic Maxwell's wave equations for various model systems. These systems are composed of two media including a transition region where the molecular environment is different from that in the bulk. The formal solutions are said to be partially non-local in space. Here the words "partially non-local in space" mean that the form of the formal solutions is expressed in terms of different spatial points. One is an observation point in either medium and the other is a point in the material medium. In the second section we will consider the system of a material bounded by a vacuum with an electromagnetic wave incident on the material from a source point in the vacuum. Our results on reflection and refraction contain physical surface effects due to the transition region in the material. In the limit of the vanishingly small thickness of the transition region, our results can be reduced to those which have appeared in the literature. The remainder of this chapter is organized 
as follows: In section 3, we replace the region of vacuum by another simple non-absorbing dielectric and place the source of the incident wave in it. At the interface between the two media, the transition region is also assumed. As a result of this replacement, we will not only be able to examine our formal solutions from various physical viewpoints, but will also be able to apply them to various important real systems, such as, a solid-gas interface. This case will be discussed in detail in later chapters (see Chapters IV, V and VI). In section 4, as a simple application of the extinction theorem, a "local effective field" is included in our formal solution in order to derive the Lorentz-Iorenz formula. The detailed analysis of the extinction theorem such as the precise phase relation between the incident and induced waves will be discussed in the third chapter.

\section{MATERIAL BOUNDED BY A VACUUM}

We choose the boundary surface $S+$ to be the vacuum boundary at which atoms (or molecules) gradually start to form a material medium. This transition region of volume $V_{ \pm}$extends to another boundary $S-$ inside the material, where the material can be regarded as linear, isotropic, and homogeneous. We denote by $\mathrm{V}$ this material region bounded by the surface $\mathrm{S}-$. We also denote by $\widetilde{V}$ the vacuum space surrounding the surface $S+$ (see Fig. 2.1).

In this system, electromagnetic radiation is incident 
MATERIAI BOUNDED BY A MEDIUM Mo

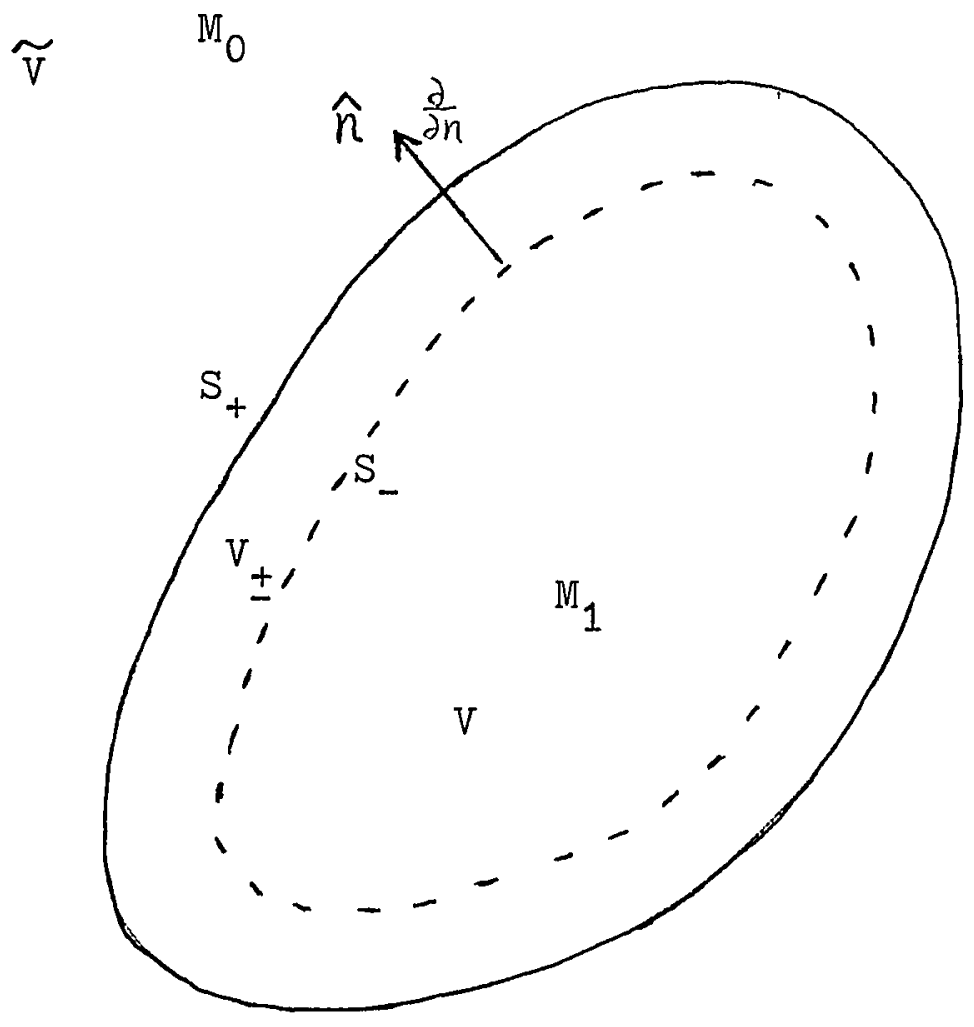

$\frac{\partial}{\partial n}$ denotes differentiation along the outward normal to $S_{-}$.

Fig. 2.1 
on the material from the vacuum. In the material it induces microscopic charges, currents, electric and magnetic dipole moments, etc. Their appropriately averaged field quantities obey the macroscopic Maxwell equations. In terms of the usual notations, they are;

$$
\begin{aligned}
& \nabla \cdot \vec{E}(\vec{r}, t)=4 \pi\langle\rho(\vec{r}, t)\rangle, \\
& \nabla \times \vec{E}(\vec{r}, t)=-\frac{1}{c} \frac{\partial}{\partial t} \vec{B}(\vec{r}, t), \\
& \nabla \cdot \vec{B}(\vec{r}, t)=0, \\
& \nabla \times \vec{B}(\vec{r}, t)=\frac{4 \pi}{c}\langle\rho(\vec{r}, t) \vec{v}(\vec{r}, t)\rangle+\frac{1}{c} \frac{\partial}{\partial t} \vec{E}(\vec{r}, t) .
\end{aligned}
$$

Here $\langle\rho\rangle$ and $\langle\hat{\vec{v}}\rangle$ are a macroscopic average of the microscopic charge density of free and bound charges and their current densities in the material respectively. In the region $V$, the macroscopic quantities are obtained by an appropriate spatial average which is described in the text of J. D. Jackson, 1975, but in the transition region $V_{ \pm}$ a different averaging process is used (see Chapter IV). In the material, we assume the following relation for $\langle\rho\rangle$,

$$
\langle\rho(\vec{r}, t)\rangle=\rho(\vec{r}, t)-\nabla \cdot \vec{p}(\vec{r}, t),
$$

where $\rho(\vec{r}, t)$ is the macroscopic charge density of free electrons and $\vec{P}(\vec{r}, t)$ is the macroscopic polarization of molecular electric dipole moments. Similarly we assume the following expression for $\langle p \overrightarrow{\vec{v}}\rangle$.

$$
\langle\rho(\vec{r}, t) \vec{v}(\vec{r}, t)\rangle=\vec{j}(\vec{r}, t)+\frac{\partial}{\partial t} \vec{P}(\vec{r}, t)+C \nabla \times \vec{M}(\vec{r}, t),
$$

where $\vec{j}(\vec{r}, t)$ is the macroscopic current density of free electrons and $\vec{M}(\vec{r}, t)$ is the macroscopic magnetization of the molecular magnetic moments (intrinsic magnetic moments 
can be added).

Noting that the source current density, $\vec{J}$, is in the vacuum, we write the Maxwell's equation for the entire region in terms of a single equation,

$$
\nabla \times \vec{H}(\vec{r}, t)=\frac{4 \pi}{c} \vec{J}(\vec{r}, t)+\frac{4 \pi}{c}\left(\frac{\partial}{\partial t} \vec{P}(\vec{r}, t)+\vec{j}(\vec{r}, t)\right)+\frac{1}{c} \frac{\partial}{\partial t} \vec{E}(\vec{r}, t) .
$$

Then the wave equations for the entire region are;

$$
\begin{gathered}
\nabla \times(\vec{N} \times \vec{E}(\vec{r}, t))+\frac{1}{c^{2}} \frac{\partial^{2}}{\partial t^{2}} \vec{E}(\vec{r}, t)=-\frac{4 \pi}{c^{2}}\left(\frac{\partial}{\partial t} \vec{j}(\vec{r}, t)+\frac{\partial}{\partial t} \vec{j}(\vec{r}, t)+\frac{\partial^{2}}{\partial t^{2}} \vec{P}(\vec{r}, t)\right)-\frac{4 \pi}{c} \nabla \times \frac{\partial}{\partial t} \vec{M}(\vec{r}, t),(2.1 \mathrm{a}) \\
\nabla \times(\nabla \times \vec{H}(\vec{r}, t))+\frac{1}{c^{2}} \frac{\partial^{2}}{\partial t^{2}} \vec{H}(\vec{r}, t)=\frac{4 \pi}{c} \nabla \times\left(\vec{j}(\vec{r}, t)+\vec{J}(\vec{r}, t)+\frac{\partial}{\partial t} \vec{P}(\vec{r}, t)\right)-\frac{4 \pi}{c^{2}} \frac{\partial^{2}}{\partial t^{2}} \vec{M}(\vec{r}, t) .(2.1 \mathrm{~b}) \\
\text { When the material is dispersive, we must transform }
\end{gathered}
$$
the field quantities from local forms to non-local forms (in time). This is done by using their Fourier transforms with respect to time. The Fourier integral for the electric field $\vec{E}(r, t)$ is expressed as

$$
\vec{E}(\vec{r}, t)=\frac{1}{2 \pi} \int_{-\infty}^{+\infty} d \omega \vec{E}(\vec{r}, \omega) e^{-i \omega t}
$$

where its Fourier vector amplitude $\vec{E}(r, \omega)$ is given as

$$
\vec{E}(\vec{r}, \omega)=\int_{-\infty}^{+\infty} d t \vec{E}(\vec{r}, t) e^{i \omega t} .
$$

Similar expressions for the other field quantities also hold. Thus, in terms of Fourier vector amplitudes of angular frequency $\omega$, the above wave equations become;

$$
\begin{aligned}
& \nabla \times(\nabla \times \vec{E}(\vec{r}, \omega))-\frac{\omega^{2}}{c^{2}} \vec{E}(\vec{r}, \omega)=4 \pi\left(i \frac{\omega}{c^{2}} \vec{J}(\vec{r}, \omega)+i \frac{\omega}{c^{2}} \vec{J}(\vec{r}, \omega)+\frac{\omega^{2}}{c^{2}} \vec{P}(\vec{r}, \omega)+i \frac{\omega}{c} \nabla \times \vec{M}(\vec{r}, \omega)\right),(2.2 a) \\
& \nabla \times(\nabla \times \vec{H}(\vec{r}, \omega))-\frac{\omega^{2}}{c^{2}} \vec{H}(\vec{r}, \omega)=4 \pi\left(\frac{1}{c} \nabla \times \vec{J}(\vec{r}, \omega)+\frac{1}{c} \nabla \times \vec{J}(\vec{r}, \omega)-i \frac{\omega}{c} \nabla \times \vec{P}(\vec{r}, \omega)+\frac{\omega^{2}}{c^{2}} \vec{M}(\vec{r}, \omega)\right) .(2.2 b) \\
& \text { We assume that the source } \vec{J}(r, \omega) \text { is far away from }
\end{aligned}
$$

the boundary of the material. Then the formal solutions of the above wave equations, Eqs. $(2.2 a)$ and $(2.2 b)$, can be obtained by using the transverse dyadic Green's function. The Green's function is defined, noting that the free space wave number is $k_{0}=\frac{\omega}{\mathrm{c}}$, as 


$$
\mathbb{G}\left(\vec{r} \vec{r}^{\prime} ; k_{0}\right)=g\left(\vec{r} ; \vec{r}^{\prime} ; k_{0}\right) \mathbb{U}-\frac{1}{k_{0}^{2}} \nabla \nabla^{\prime} \dot{y}\left(\vec{r} \mid \vec{r}^{\prime} ; k_{0}\right),
$$

where $\mathbb{U}$ is the unit dyadic and $g\left(\vec{r} \mid \vec{r}^{\prime} ; k_{0}\right)=\frac{e^{i k_{0}\left|\vec{r}-\vec{r}^{\prime}\right|}}{|\vec{r}-\vec{r}|}$ is an outgoing free space Green's function. In Eq. (2.3), the second term on the right expresses the longitudinal part of the dyadic Green's function (see the text of P. M. Morse and H. Feshbach, 1953). The two Green's functions obey the following equations respectively,

$$
\begin{aligned}
& {\left[-\nabla \times \nabla \times+k_{0}^{2}\right\} G\left(\vec{r} \mid \vec{r}^{\prime} ; k_{0}\right)=-4 \pi \delta\left(\vec{r}-\vec{r}^{\prime}\right) \mathbb{U},} \\
& {\left[\nabla^{2}+k_{0}^{2}\right] g\left(\vec{r} \mid \vec{r}^{\prime} ; k_{0}\right)=-4 \pi \delta\left(\vec{r}-\vec{r}^{\prime}\right),}
\end{aligned}
$$

where $\delta\left(\vec{r}-\vec{r}^{\prime}\right)$ is the Dirac delta function. We note that for the case of $\vec{r} \neq \vec{r}^{\prime}$, the symmetry property of Eq. (2.4a) with respect to $\vec{r}$ and $\vec{r}^{\prime}$ gives the following relation;

$$
\begin{aligned}
\mathbb{C}\left(\vec{r} ; \vec{r}^{\prime} ; k_{0}\right) & =\frac{1}{k_{0}^{2}} \vec{\nabla}^{\prime} \times \nabla^{\prime} \times\left(\tau\left(\vec{r} \mid \vec{r}^{\prime} ; k_{0}\right)\right. \\
& =\frac{1}{k_{0}^{2}} \nabla^{\prime} \times \nabla^{\prime} \times\left[\mathbb{U} g\left(\vec{r} \mid \vec{r}^{\prime} ; k_{0}\right)-\frac{1}{k_{0}^{2}} \nabla \nabla^{\prime} g\left(\vec{r} \mid \vec{r}^{\prime} ; k_{0}\right)\right] \\
& =\frac{1}{k_{0}^{2}} \nabla^{\prime} \times \nabla^{\prime} \times \mathbb{U} g\left(\vec{r} \mid \vec{r}_{r}^{\prime} k_{0}\right),\left(\vec{r} \neq \vec{r}^{\prime}\right) .
\end{aligned}
$$

The formal solution of Eq. (2.2a) can now be obtained by using the following well known method. First we take the dot product of $G\left(\vec{r} \mid \vec{r}^{\prime} ; k_{0}\right)$ with every term of Eq. (2.2a) from its left, similarly we take the dot produce of $\vec{E}(\vec{r}, \omega)$ with every term of Eq. (2.4a) from its right, then we add the resulting equations noting that by definition $\vec{J}(\vec{r}, \omega)$ vanishes in $V_{ \pm}$and that $\delta(\vec{r}-\vec{r})=0$ for $\vec{r} \neq \vec{r}^{\prime}$. Integrating (with respect to $\vec{r}$ ) the resulting equation over the volume, $\widetilde{V}+V_{ \pm}$, we find the solution to be,

$$
\begin{aligned}
& \frac{i k_{0}}{C} \int_{\vec{v}} d^{3} r\left\{\left(\vec{r}, \nabla^{\prime} ; k_{0}\right) \cdot \vec{J}(\vec{r}, \omega)+\int_{V_{ \pm}} d \vec{r} G\left(\vec{r} \vec{r}^{\prime} ; k_{0}\right) \cdot\left(i \frac{k_{0}}{i} \vec{j}(\vec{r}, \omega)+k_{0}^{2} \vec{E}(\vec{r}, \omega)+i k_{0} \nabla \times \vec{r} \vec{r}(\vec{r}, \omega)\right)+\right. \\
& \text { I }
\end{aligned}
$$

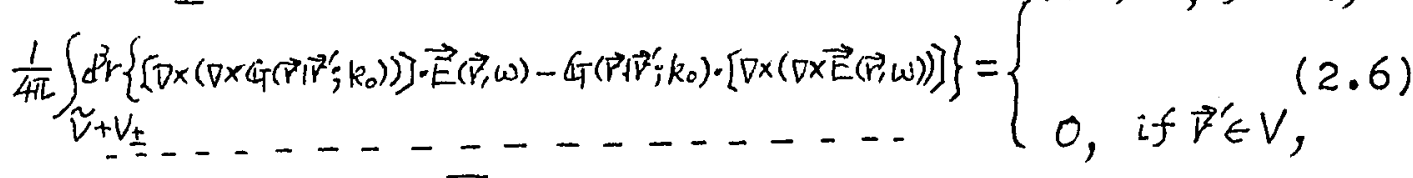


where $\vec{r}^{\prime}=\vec{r}^{\prime}$ on the lefthand side if $\vec{r}^{\prime} \in \widetilde{V}$. Also $\vec{r}^{\prime}=\vec{r}^{\prime}$ if $\vec{r}^{\prime} \in V$. On the left-hand side of $\mathrm{Eq} \cdot(2.6)$, the first, second and third terms are denoted respectively by I, II, and III for later reference. Term $I$ is related with the source. Term II contains the effect of the transition region. Term III will become physically more tractable if we transform it into a surface integral over $S_{-}$. This can be done by noting that the integrand of term III can be written,

$$
\left[\nabla \times(\nabla \times(\leftarrow)] \cdot \vec{E}-G \cdot[\nabla \times(\nabla \times \vec{E})]=\nabla \cdot\left[\left(\nabla \times(\dot{T}) \times \vec{E}+4_{T} \times(\nabla \times \vec{E})\right],\right.\right.
$$

where we have used the vector relations,

$$
\begin{aligned}
& \nabla \cdot\{(\pi \times(\nabla \times \vec{E})]=(\nabla \times(斤) \cdot(\nabla \times \vec{E})-(T \cdot[\nabla \times(\nabla \times \vec{E})], \\
& \nabla \cdot\{(\nabla \times(T) \times \vec{E}]=[\nabla \times(\nabla \times(T)] \cdot \vec{E}-(\nabla \times(T) \cdot(\nabla \times \vec{E}) .
\end{aligned}
$$

Then term III leads to

$$
\begin{aligned}
\text { III } & =\frac{1}{4 \pi} \int_{S_{-}} d S(-\hat{n}) \cdot\left[-\vec{E}(\vec{r}, \omega) \times\left(\nabla \times G\left(\vec{r} \mid \vec{r}^{\prime} ; k_{0}\right)\right)+G\left(\vec{r} \mid \vec{r}^{\prime} ; k_{0}\right) \times(\nabla \times \vec{E}(\vec{r}, \omega))\right] \\
& =\frac{1}{4 \pi} \int_{S} d S\{\hat{n} \times \vec{E}(\vec{r}, \omega)) \cdot\left(\nabla \times G\left(\vec{r} \mid \vec{r}^{\prime} ; k_{0}\right)\right)+G\left(\vec{r} \mid \vec{r}^{\prime} ; k_{0}\right) \cdot[\hat{n} \times(\nabla \times \vec{E}(\vec{r}, \omega))],
\end{aligned}
$$

where $\hat{n}$ is the outward normal unit vector to the closed surface $S_{-}$. Using the relation established in Eq. (2.5), term III can now be written as

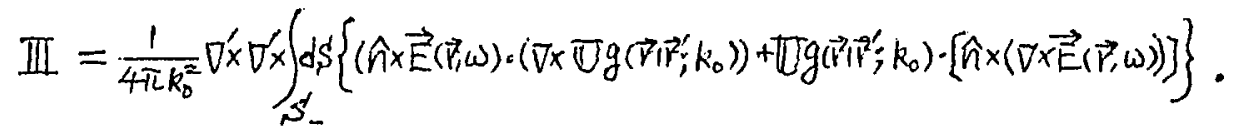

Any Cartesian component of the integrand in Eq. (2.7) can be written

$$
\{(\hat{n} \times \vec{E}) \cdot(\nabla \times \nabla g)+\nabla g \cdot[\hat{n} \times(\nabla \times \vec{E})]\}_{\alpha \alpha}=g n_{\beta} \nabla_{\alpha} E_{\beta}-g n_{\beta} \nabla_{\beta} E_{\alpha}+E_{\alpha} \eta_{\beta}\left(\nabla_{\beta} g\right)-n_{\alpha} E_{\beta}\left(\nabla_{\beta} g\right), \quad(2.8)
$$
where $\alpha$ and $\beta$ are any of the Cartesian co-ordinates $x, y$ and $\mathbf{z}$, and the Einstein summation convention is used. $\nabla_{\beta}$ denotes the partial differentiation with respect to the coordinate $\beta$. The last term in Eq. (2.8) may be written as, $n_{\alpha} E_{\beta}\left(\nabla_{\beta} g\right)=n_{\alpha}\left(\nabla_{\beta} E_{\beta} g\right)-n_{\alpha} g \nabla_{\beta} E_{\beta}$ 


$$
=n_{\alpha}\left(\nabla_{\beta} E_{\beta} g\right)-n_{\alpha} g\left\{4 \pi\left(\frac{1}{i \omega} \nabla \cdot \vec{j}-\nabla \cdot \vec{P}\right)\right\},
$$

where we have used the continuity equation for the macroscopic charge density $P(\vec{r}, \omega)$ of free electrons, the current density $\vec{j}(\vec{r}, \omega)$ and also the polarization vector field $\vec{P}(r, \omega)$ defined through the relation,

$$
\langle\rho(\vec{r}, \omega)\rangle=\rho(\vec{r}, \omega)-\nabla \cdot \vec{P}(\vec{r}, \omega) \text {. }
$$

Thus Eq. (2.7) leads to

$$
\begin{aligned}
\text { III } & =\frac{1}{4 \pi k_{0}^{2}} \nabla^{\prime} \times \nabla^{\prime} \times \int_{S} d S\left\{\vec{E}(\vec{r}, \omega) \frac{\partial}{\partial n} g\left(\vec{r} \mid \vec{r} ; k_{0}\right)-g\left(\vec{r} \mid \vec{r}^{\prime} ; k_{0}\right) \frac{\partial}{\partial n} \vec{E}(\vec{r}, \omega)\right\} \\
& +\frac{1}{4 \pi k_{0}^{2}} \nabla \times \nabla \int_{S}^{\prime} d S\left\{g\left(\vec{r} \mid \vec{r}^{\prime} ; k_{0}\right) \nabla(\hat{r} \cdot \vec{E}(\vec{r}, \omega))-\hat{n}\left(\nabla \cdot \vec{E}(\vec{r}, \omega) g\left(\vec{r} \mid \vec{r}^{\prime} ; k_{0}\right)\right)\right\} \\
& +\frac{1}{k_{0}^{2}} \nabla^{\prime} \times \nabla^{\prime} \times \int_{S_{-}} d S^{\frac{\hat{n}}{i \omega}} g\left(\vec{r} \mid \overrightarrow{r^{\prime}} ; k_{0}\right) \nabla \cdot \vec{j}(\vec{r}, \omega)-\frac{1}{k_{0}^{2}} \nabla^{\prime} \times \nabla^{\prime} \times \int_{S_{-}} d S \hat{n} g\left(\vec{r} \mid \vec{r}^{\prime} ; k_{0}\right) \nabla \cdot \vec{P}(\vec{r}, \omega) .
\end{aligned}
$$

The second term in the above equation vanishes. To see this, consider the following surface integral,

$$
\begin{aligned}
& \int_{S_{-}} d s \hat{n}\{\nabla \cdot(g \vec{E})\}=-\int_{\hat{V}+V_{ \pm}^{3}}\{\nabla[\nabla \cdot(\vec{E} g)]\} \\
& =-\int_{\hat{y}+V_{+}} d^{3} r\{(\nabla \cdot \nabla)(\vec{E} g)+\nabla \times(\nabla \times \vec{E} g)\} \\
& \left.=\int_{S_{-}} d s \hat{n} \hat{n} \cdot \nabla(\vec{E} g)+\hat{n} \times(\nabla \times \vec{E} g)\right\} \\
& =\int_{S_{-}}^{d s}\{\nabla(\hat{n} \cdot \vec{E} g)\} \\
& =\int_{S_{-}} d s\{g \nabla(\hat{n} \cdot \vec{E})+\hat{n} \cdot \vec{E} \nabla g\} .
\end{aligned}
$$

We then find the second term to be,

$$
\begin{aligned}
& \frac{1}{4 \pi k_{0}^{2}} \nabla^{\prime} \times \nabla^{\prime} \int_{S_{0}} d s\left\{g\left(\vec{r} \vec{r}_{;}^{\prime} k_{0}\right) \nabla(\hat{n} \cdot \vec{E}(\vec{r}, \omega))-g\left(\vec{r} \vec{P} ; k_{0}^{\prime}\right) \nabla\left(\hat{n} \cdot \vec{E}(\vec{r}, \omega)-\hat{n} \cdot \vec{E}(\vec{r}, \omega) \nabla g\left(\vec{r}\left(\vec{r} ; k_{0}\right)\right\}\right.\right. \\
& =\frac{1}{4 \pi k_{e}^{2}} \nabla^{\prime} \times \nabla^{\prime} \times \int_{S_{-}} d S\left\{\hat{n} \cdot \vec{E}(\vec{r}, \omega) \nabla^{\prime} g\left(\vec{p}\left(\vec{r}^{\prime} ; k_{0}\right)\right\}=0\right. \text {. }
\end{aligned}
$$

Using Eq. (2.5), we rewrite term II in Eq. (2.6), in terms of the free space Green's function $g\left(\vec{r} \mid \vec{r}^{\prime} ; k_{0}\right)$. Then Eq. (2.6) becomes,

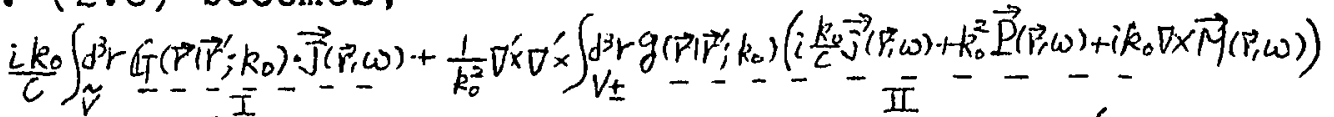

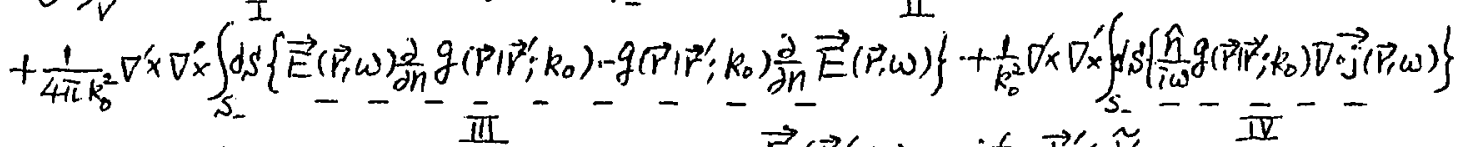

$$
\begin{aligned}
& -\frac{1}{k_{0}^{2}} \nabla^{\prime} \times \nabla^{\prime} \times \int_{S_{-}} d s\left\{\hat{n} g\left(\vec{r} \mid \vec{r}^{\prime} ; k_{0}\right) \nabla \cdot \vec{B}(\vec{r}, \omega)\right\}=\left\{\begin{array}{cc}
\vec{E}(\vec{r} ;, \omega), & \text { if } \vec{r}^{\prime} \in \tilde{V} . \\
0, & \text {, if } \vec{r}^{\prime} \in V .
\end{array}\right.
\end{aligned}
$$


The process of obtaining the formal solution of Eq. $(2.2 \mathrm{~b})$ is exactly the same as that of Eq. (2.2a). The result is,

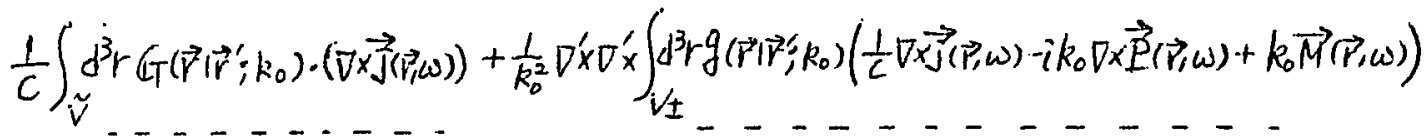

$$
\begin{aligned}
& \mathrm{I}^{\prime} \cdots \text { II }^{\prime} \cdots \cdots \\
& +\frac{1}{4 \pi k_{0}^{2}} \nabla^{\prime} \times \nabla^{\prime} \times \int_{S_{-}} d s\left\{\vec{H}(\vec{r}, \omega) \frac{\partial}{\partial n} g\left(\vec{r} \vec{r}^{\prime} ; k_{0}\right)-g\left(\vec{r} \mid \vec{r}^{\prime} ; k_{0}\right) \frac{\partial}{\partial n} \vec{H}(\vec{r}, \omega)\right\} \\
& -\frac{1}{k_{0}^{2}} \nabla^{\prime} \times \nabla^{\prime} \times \int_{S_{-}} d S\left\{g\left(\vec{r} \mid \vec{r}^{\prime} ; k_{0}\right) \hat{n} \nabla \cdot \vec{M}(\vec{r}, \omega)\right\}=\left\{\begin{array}{cc}
\vec{H}(\vec{r}, \omega), \text { if } \vec{r}^{\prime} \in \widetilde{V} . \\
0, \text { if } \vec{r}^{\prime} \in V_{v} .
\end{array}\right. \\
& \text { ……… }
\end{aligned}
$$

In the volume integral. II, of Eq. (2.9), the magnetization term can be written as

$$
\begin{aligned}
& \frac{1}{k_{0}^{2}} \nabla^{\prime} \times \nabla^{\prime} \times \int_{V_{ \pm}} d^{\beta} r\left\{i k_{0} g\left(\vec{\nabla} \vec{r}^{\prime} ; k_{0}\right) \nabla \times \vec{M}(\vec{r}, \omega)\right\} \\
& =\frac{1}{k_{0}^{2}} \nabla^{\prime} \times \nabla^{\prime} \int_{V_{ \pm}}^{\prime} d^{3} r i k_{0}\left\{\nabla \times\left(\vec{M}(\vec{r}, \omega) g\left(\vec{r} \mid \vec{r}^{\prime} ; k_{0}\right)-\left(\nabla g\left(\vec{r} \mid \vec{r}^{\prime} ; k_{0}\right)\right) \times \vec{M}(\vec{P}, \omega)\right\}\right. \\
& =\frac{1}{k_{0}^{2}} \nabla^{\prime} \times \nabla^{\prime} \times \int_{S_{-}} d s\left\{i k_{0} g\left(\vec{r} \overrightarrow{r^{\prime}} ; k_{0}\right)(-\hat{n}) \times \vec{M}(\vec{r}, \omega)\right\}-\frac{1}{k_{0}^{2}} \nabla^{\prime} \nabla^{\prime} \times \int_{V_{ \pm}} d^{3} r\left\{i k_{0}\left[\nabla g\left(\vec{P} \vec{r}^{\prime} ; k_{0}\right)\right] \times \vec{M}(\vec{P}, \omega)\right\} .
\end{aligned}
$$

Similarly the current density and the polarization vector terms in II' of Eq. (2.10) become respectively,

$$
\begin{aligned}
& \frac{1}{k_{0}^{2}} \nabla^{\prime} \times \nabla^{\prime} \times \int_{V_{ \pm}} d^{3} r\left\{\frac{1}{c} g\left(\vec{r} \mid \vec{r}^{\prime} ; k_{0}\right) \nabla \times \vec{j}(\vec{r}, \omega)\right\} \\
= & \left.\frac{1}{k_{0}^{2}} \nabla^{\prime} \times \nabla^{\prime} \times \int_{S_{-}} d S\left\{\frac{1}{c} g\left(\vec{r} \mid \vec{r}^{\prime} ; k_{0}\right)(-\hat{n}) \times \vec{j}(\vec{r}, \omega)\right\}-\frac{1}{k_{0}^{2}} \nabla^{\prime} \times \nabla^{\prime} \times \int_{V_{ \pm}} d^{3}\right\}\left\{\left[\nabla g\left(\vec{r} \mid \vec{r} ; k_{0}\right)\right] \times \frac{1}{c} \vec{j}(\vec{r}, \omega)\right\}(2.12)
\end{aligned}
$$

and

$$
\begin{aligned}
& \frac{r}{k_{0}^{2}} \nabla^{\prime} \times \nabla^{\prime} \times \int_{V_{ \pm}} d^{3} r\left\{\left(-i k_{0}\right) g\left(\vec{r} \mid \vec{r}^{\prime} ; k_{0}\right) \nabla \times \vec{P}(\vec{r}, \omega)\right\} \\
= & \frac{1}{k_{0}^{2}} \nabla^{\prime} \times \nabla^{\prime} \times \int_{S_{-}} d S\left\{i k_{0} g\left(\vec{r} \mid \vec{r}^{\prime} ; k_{0}\right) \hat{n} \times \vec{P}\left(\overrightarrow{r^{\prime}}, \omega\right)\right\}+\frac{1}{k_{0}^{2}} \nabla^{\prime} \times \nabla^{\prime} \times \int_{V_{ \pm}} d^{3} r\left\{i k_{0}\left[\nabla g\left(\vec{r} \mid \vec{r}^{\prime} ; k_{0}\right)\right] \times \vec{P}(\vec{r}, \omega)\right\}(2.13)
\end{aligned}
$$

Thus the final forms of the formal solutions, Eqs. (2.9) and $(2.10)$ are; 


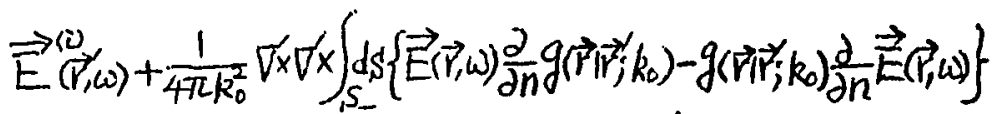

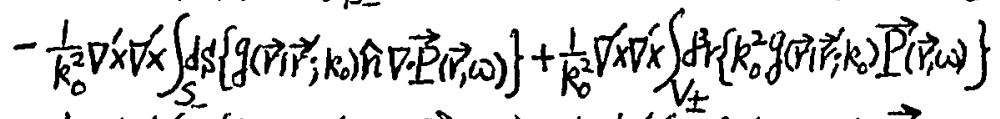

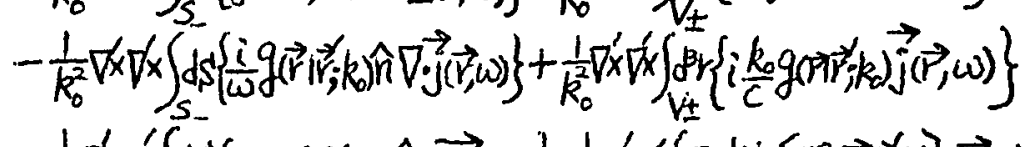

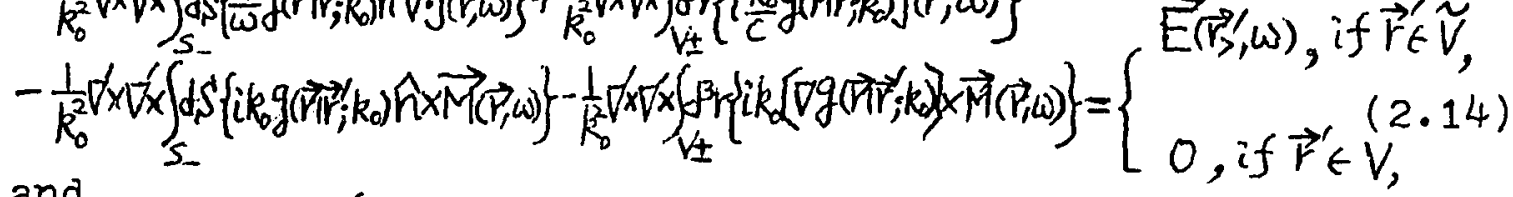

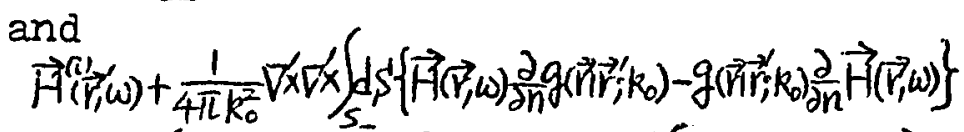

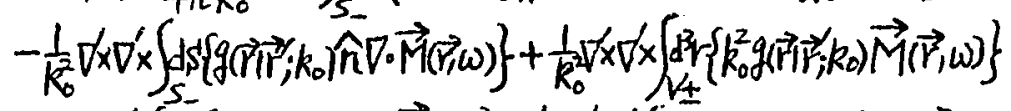

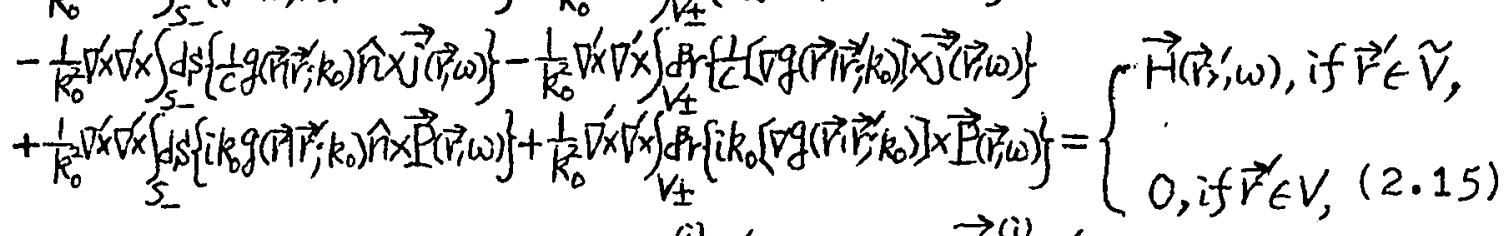

where the incident waves, $\vec{E}^{(i)}(\vec{r}, \omega)$ and $\vec{H}^{(i)}(\vec{r}, \omega)$, are defined as

and

$$
\vec{E}^{(i)}\left(\vec{r}^{\prime}, \omega\right)=\frac{i k_{0}}{C} \int_{\tilde{v}} d^{3} r \llbracket\left(\vec{r} \vec{r}^{\prime} ; k_{0}\right) \cdot \vec{J}(\vec{r}, \omega)
$$

$$
\vec{H}^{(i)}\left(\vec{r}^{\prime}(\omega)=\frac{1}{C} \int_{\tilde{v}} d^{\beta} r G\left(\vec{r} \mid \vec{r}^{\prime} ; k_{0}\right) \cdot(\nabla \times \vec{J}(\vec{r}, \omega)) .\right.
$$

In these formal solutions, Eqs. (2.14) and (2.15), the physical surface effects appear as the volume integrals in the transition region, $V_{ \pm}$. An example of the use of the volume integrals for anomalous dispersion will be fully discussed in the next chapter.

If the boundary of the material can be assumed to be a geometrically sharp boundary $S$ and if the material can be regarded as a perfect conductor, we can intuitively see that by taking the limit as the thickness of transition volume $V_{ \pm}$approaches zero, all the volume integral terms in Eqs. (2.14) and (2.15) except for those containing the current density $\vec{j}(\vec{r}, \omega)$ vanish. Furthermore all the surface integrals in Eqs. (2.14) and (2.15) must vanish. In this 
case, Eqs. (2.14) and (2.15) reduce to

$$
\begin{aligned}
& \vec{E}(\vec{r}, \omega)+\frac{1}{k_{0}^{2}} \nabla^{\prime} \times \nabla^{\prime} \int_{s} d S\left\{i \frac{k_{0}}{C} g\left(\vec{r} \mid \vec{r}^{\prime} ; k_{0}\right) \vec{j}^{(s)}(\vec{r}, \omega)\right\}=\left\{\begin{array}{l}
\vec{E}\left(\vec{r}^{\prime}, \omega\right), \text { if } \vec{r}^{\prime} \in \widetilde{V}, \\
0, \text { if } \vec{r}^{\prime} \in V,
\end{array}\right. \\
& \vec{H}^{(i)}\left(\vec{r}^{\prime}, \omega\right)+\frac{1}{k_{0}^{2}} \nabla^{\prime} \times \nabla^{\prime} \int_{S} d S\left\{i k_{0}\left[\nabla g\left(\vec{r} \mid \vec{r}^{\prime} ; k_{0}\right) \times \vec{j}^{(s)}(\vec{r}, \omega)\right\}=\left\{\begin{array}{l}
\vec{H}\left(\vec{r}^{\prime}, \omega\right), \text { if } \vec{r}^{\prime} \in \widetilde{V}, \\
0, \text { if } \vec{r}^{\prime} \in V,
\end{array}\right.\right.
\end{aligned}
$$

where the surface current density is denoted by $\vec{j}(s)(\vec{r}, \omega)$. It can be defined by integrating the volume current density of $\vec{j}(\vec{r}, \omega)$ over a vanishingly small thickness along the normal to the surface $S$. In deriving these surface current contributions, we can also use the following expression (see the text of M. Born and E. Wolf, 1975b),

$$
\vec{j}(\vec{r}, \omega)=\vec{j}(s)(\vec{r}, \omega)|\nabla \vec{F}(\vec{r})| \delta(\vec{F}),
$$

where $F(\vec{r})=0$ defines the boundary surface $S$. The condition, $F(\vec{r})>0$, refers to the material region and $\delta(F)$ is the Dirac delta function.

If the material is not a perfect conductor, all the volume integrals in Eqs. (2.14) and (2.15) vanish with the surface integrals at $S$ - remaining.

We can compare our results with those which have appeared in the literature. In the work of D. N. Pattanayak and E. Wolf (1972) and E. Wolf (1976), they did not introduce the transition region $V_{ \pm}$so that their results are for the sharp boundary. In order to get their equation similar to our Eq. (2.6), they took the volume integral over the volume $\widetilde{V}$ so that their boundary surface corresponds to our surface $S+$. The field quantities at $S+$ are then for the vacuum. In order to relate the surface integrals at $S_{+}$ 
to those at $S_{-}$just inside of the sharp boundary of the material, they used the following well known continuity conditions;

$$
\begin{aligned}
& \hat{n} \times\left[\vec{E}^{(+)}(\vec{r}, \omega)-\vec{E}^{(-)}\left(\vec{r}_{2}, \omega\right)\right]=0, \\
& \hat{n} \times\left[\vec{H}^{(+)}\left(\vec{r}_{\geqslant}, \omega\right)-\vec{H}^{(-)}\left(\vec{r}_{<}, \omega\right)\right]=\left\{\begin{array}{c}
\frac{4 \pi}{i} \vec{j}((\vec{r}), \omega), \text { if the conductor is perfect, } \\
0 \text {, otherwise, }
\end{array}\right.
\end{aligned}
$$

where the subscripts $(+)$ and $(-)$ refer to surfaces $S_{+}$and $S$ - respectively. Therefore, although the method is different, our results, Eqs. (2.14) and (2.15) in the limit of the sharp boundary agree exactly with those of the above investigators. On the other hand, J. deGeode and P. Mazur (1972) considered the transition region, $V_{ \pm}$, for conductors, although their results are for a sharp boundary. In their approach to the sharp boundary, they took a vanishingly small thickness of the transition region so that the surface current appears from the singularity of $c \nabla \times \vec{M}(\vec{r}, \omega)$ at the sharp boundary. Namely, they expressed the magnetization vector, $\vec{M}(\vec{r}, \omega)$, for the sharp boundary, as

$$
\vec{M}(\vec{r}, \omega)=U(F) \vec{M}(\vec{r}, \omega) \text {, }
$$

where $U(F)$ is the Heaviside unit step function defined as,

$$
U(F)= \begin{cases}0, & \text { if } F(\vec{r})<0 . \\ 1, & \text { if } F(\vec{r})>0 .\end{cases}
$$

Here $F(\vec{r})=0$ defines the boundary surface. Then they found the following,

$$
C \nabla \times \vec{M}(\vec{r}, \omega)=U(\vec{H}) C \nabla \times \vec{M}(\vec{r}, \omega)+C \delta(F)(\nabla \vec{H}(\vec{r})) \times \vec{M}(\vec{r}, \omega) .
$$

Using the outward normal unit vector, $\hat{n}=-\frac{\nabla F(\vec{r})}{|\nabla \vec{H}(\vec{r})|}$, this can be written as 
$C \nabla \times \vec{M}(\vec{r}, \omega)=U(\bar{H}) C \nabla \times \vec{M}(\vec{r}, \omega)-\delta(\vec{H})|\nabla \bar{F}(\vec{r})| C \hat{n} \times \vec{M}(\vec{r}, \omega)$.

The second term of this equation is related to the surface current density. It corresponds to the first term in $\mathrm{Eq}$. (2.11) of our model.

3. MATERIAL BOUNDED BY ANOTHER MATERIAL MEDIUM

In this section, let us consider the solution of Maxwell's wave equations for the case where the previous vacuum region is replaced by a material medium. This medium will be referred to as $\mathrm{M}_{0}$ and the bounded material as $M_{1}$. We assume that the medium $M_{0}$ is a linear, isotropic, homogeneous, and non-absorbing dielectric. The source, $\vec{J}(\vec{r}, \omega)$, is in the medium $\mathbb{M}_{0}$. Since $\mathbb{M}_{0}$ is no longer a vacuum, the previous Maxwell's equations are modified by adding an induced macroscopic charge density and a current density of the medium $\mathrm{M}_{0}$.

$$
\begin{aligned}
& \nabla \cdot \vec{E}(\vec{r}, \omega)=\left\{\begin{array}{l}
4 \pi\left\langle\rho_{0}(\vec{r}, \omega)\right\rangle+\frac{4 \pi}{i \omega} \nabla \cdot \vec{J}(\vec{r}, \omega), \\
4 \pi\langle\rho(\vec{r}, \omega)\rangle, \text { in } M_{0},
\end{array}\right. \\
& \nabla \times \vec{E}(\vec{r}, \omega)=i k_{0} \vec{B}(\vec{r}, \omega) \\
& \nabla \cdot \vec{B}(\vec{r}, \omega)=0 \\
& \nabla \times \vec{B}(\vec{r}, \omega)=\left\{\begin{array}{l}
\frac{4 \pi}{C}\left(\left\langle p_{0}(\vec{r}, \omega) \overrightarrow{U_{0}}(\vec{r}, \omega)\right\rangle+\vec{J}(\vec{r}, \omega)\right)-i k_{0} \vec{E}(\vec{r}, \omega), \text { in } M_{0}, \\
\frac{4 \pi}{C}(\langle\rho(\vec{r}, \omega) \vec{U}(\vec{r}, \omega)\rangle)-i k_{0} \vec{E}(\vec{r}, \omega), \text { in } M_{2},
\end{array}\right.
\end{aligned}
$$

where the subscript " 0 " refers to the medium $M_{0}$ in which the source $\vec{J}(\vec{r}, \omega)$ is located ( $k_{0}$ still stands for the free space wave number).

From the assumptions made for the media $M_{0}$ and $M_{1}$, we have their macroscopic charge densities, 


$$
\left\langle\rho_{0}(\vec{r}, \omega)\right\rangle=-\nabla \cdot \vec{P}_{c}(\vec{r}, \omega)
$$

and

$$
\langle\rho(\vec{r}, \omega)\rangle=\rho(\vec{r}, \omega)-\nabla \cdot \vec{P}(\vec{r}, \omega)
$$

for $M_{0}$ and $M_{1}$ respectively. Here we note that there are no free charges in $\mathrm{N}_{0}$ except for those contributing to the source term $\vec{J}(\vec{r}, \omega)$. Similarly, their macroscopic current densitites can be written as

$$
\left\langle\rho_{0}\left(\vec{r}_{1} \omega\right) \vec{U}_{0}\left(\vec{r}_{r} \omega\right)\right\rangle=-i \omega \vec{B}_{0}(\vec{r}, \omega)+\left(\nabla \times \vec{M}_{0}(\vec{r}, \omega)\right.
$$

for the medium $\mathrm{M}_{0}$, and

$$
\langle P(\vec{r}, \omega) \vec{U}(\vec{r}, \omega)\rangle=\vec{j}(\vec{r}, \omega)-i \omega \vec{P}(\vec{r}, \omega)+(\nabla \times \vec{M}(\vec{r}, \omega)
$$

for the medium $M_{1}$.

Since the medium $\mathbb{M}_{0}$ is linear, isotropic and homogeneous, we assume the following relations,

$$
\begin{aligned}
& \vec{D}(\vec{r}, \omega)=\vec{E}(\vec{r}, \omega)+4 \pi \vec{P}_{0}(\vec{r}, \omega)=\left(1+4 \pi \eta_{0}(\omega)\right) \vec{E}(\vec{r}, \omega)=\epsilon_{0}(\omega) \vec{E}(\vec{r}, \omega), \\
& \vec{B}(\vec{r}, \omega)=\vec{H}(\vec{r}, \omega)+4 \pi \vec{M}_{0}(\vec{r}, \omega)=\left(1+4 \pi \chi_{0}(\omega)\right) \vec{H}(\vec{r}, \omega)=\mu_{0}(\omega) \vec{H}(\vec{r}, \omega),
\end{aligned}
$$

where $\eta_{0}(\omega)$ and $\epsilon_{0}(\omega)$ are the electric susceptibility and the dielectric constant respectively and $\chi_{0}(\omega)$ and $\mu_{0}(\omega)$ are the magnetic susceptibility and the magnetic permeability respectively. Furthermore, the medium $\mathbb{M}_{0}$ is non-absorbing so that $\epsilon_{0}(\omega)$ and $\mu_{0}(\omega)$ are real.

We assume that the transition region $v_{ \pm}$starts at the boundary surface $S_{+}$where the material parameters are those for the bulk medium $\mathbb{M}_{0}$ and ends at the boundary surface $S$ in the medium $\mathrm{M}_{1}$. We may reconstruct the medium $\mathrm{M}_{1}$ by first filling the region $V_{+} V_{ \pm}$with the medium $\mathbb{M}_{0}$. Next we superpose "virtually induced field quantities" on the medium $\mathrm{M}_{0}$ in the region, $\mathrm{V}+\mathrm{V}_{ \pm}$. This construction can be done by re- 
writing the induced fields in $M_{1}, \vec{P}(\vec{r}, \omega)$ and $\vec{M}(\vec{r}, \omega)$, in terms of the material parameters of $M_{0}, \eta_{0}(\omega)$ and $\chi_{0}(\omega)$, and the adjustable virtual field quantities. These induced fields are

$$
\vec{P}(\vec{r}, \omega)=\vec{P}_{01}(\vec{r}, \omega)+\vec{P}(\vec{r}, \omega)-\vec{P}_{01}(\vec{r}, \omega)=\vec{P}_{01}(\vec{r}, \omega)+\vec{P}_{i}(\vec{r}, \omega)
$$

and

$$
\vec{M}(\vec{r}, \omega)=\vec{M}_{01}(\vec{r}, \omega)+\vec{M}(\vec{r}, \omega)-\vec{M}_{01}(\vec{r}, \omega)=\vec{M}_{01}(\vec{r}, \omega)+\vec{M}_{0}(\vec{r}, \omega),
$$

where $\vec{B}_{01}(\vec{r}, \omega)=\eta_{0}(\omega) \vec{E}(\vec{r}, \omega)$ and $\vec{M}_{01}(\vec{r}, \omega)=\chi_{0}(\omega) \vec{H}(\vec{r}, \omega)$ in terms of the mean fields $\vec{E}$ and $\vec{H}$ of $\mathbb{M}_{1}$, and $\vec{P}_{w}(\vec{r}, \omega)$ and $\vec{M}_{v}(\vec{r}, \omega)$ are the virtual polarization and the virtual magnetization fields respectively. The virtual fields in the transition region $V_{ \pm}$change continuously from zero at $S_{+}$ to their bulk quantities at $S$-.

Using the virtually induced fields, we obtain the following wave equations for the entire region, $\widetilde{V}+V_{ \pm}+V$,

$\nabla \times\left[\nabla \times \vec{E}\left(\overrightarrow{r_{1}}, \omega\right)\right]-k_{0}^{2} \epsilon_{0} \mu_{0} \vec{E}(\vec{r}, \omega)=i k_{0} \mu_{0}\left[\frac{4 \pi}{c} \vec{J}(\vec{r}, \omega)+\frac{4 \pi}{c} \vec{j}(\vec{r}, \omega)-4 \pi i k_{0} \vec{P}(\vec{r}, \omega)+4 \pi\left[\times \vec{M}_{0} \vec{V}_{1}, \omega\right)\right],(2.17 \mathrm{a})$

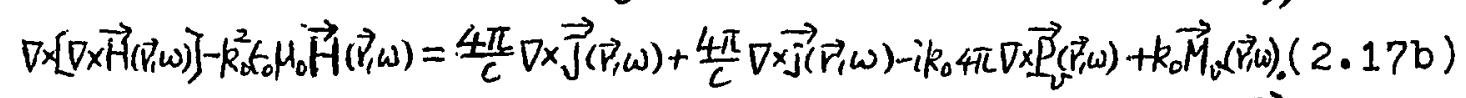

Using the transverse dyadic Green's function $\vec{G}\left(\vec{r}, \vec{r}^{\prime} ; k\right)$

in $\mathrm{M}_{0}$ and exactly the same method as in section 2, we obtain the formal solutions of the above wave equations, (2.17a) and (2.17b), in their partially non-local forms in space. The results are, noting that the wave number in the medium $M_{0}$ is $k=k_{0} \sqrt{\epsilon_{0}(\omega)}$,

$\vec{E}\left(i \vec{r}^{\prime}, \omega\right)+\frac{1}{4 \pi k^{2}} \nabla^{\prime} \nabla^{\prime} x \int_{S_{-}} \int d\left\{\vec{E}(\vec{r}, \omega) \frac{\partial}{\partial n} g\left(\vec{r} \mid \vec{r}^{\prime} ; k\right)-g\left(\vec{n} \vec{r}^{\prime} ; k\right) \frac{\partial}{\partial n} \vec{E}(\vec{r}, \omega)\right\}$

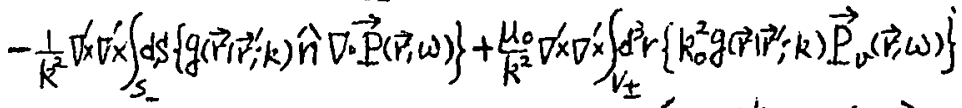

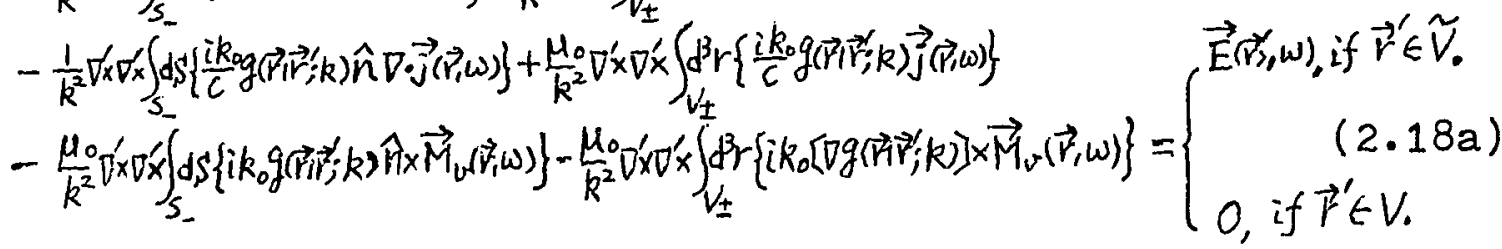


and

$$
\begin{aligned}
& \vec{H}(\vec{r}, \vec{r}, \omega)+\frac{1}{4 \pi k^{2}} \nabla^{\prime} \nabla^{\prime} \int_{S} d S\left\{\vec{H}(\vec{r}, \omega) \frac{\partial}{\partial n} g\left(\vec{r} \vec{r}^{\prime} ; k\right)-g(\vec{r} \vec{r} ; k) \frac{\partial}{\partial n} \vec{H}(\vec{r}, \omega)\right\} \\
& -\frac{1}{k^{2}} \nabla^{\prime} \times \nabla^{\prime} \int_{S_{-}} d s\left\{g\left(\vec{r} \overrightarrow{\vec{r}^{\prime}} ; k\right) \hat{n} \nabla \cdot \vec{r}(\vec{r}, \omega)\right\}+\frac{1}{k^{2}} \nabla^{\prime} \times \nabla^{\prime} \int_{V_{ \pm}} d \vec{r}\left\{k_{0}^{2} g\left(\vec{r} \overrightarrow{\vec{r}^{\prime}} ; k\right) \overrightarrow{M_{v}}(\vec{r}, \omega)\right\}
\end{aligned}
$$

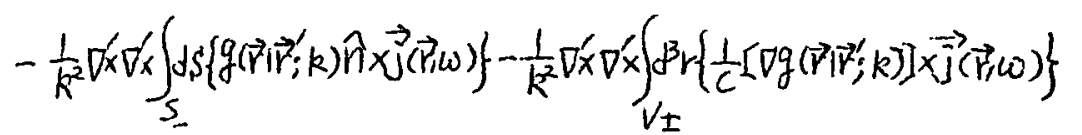

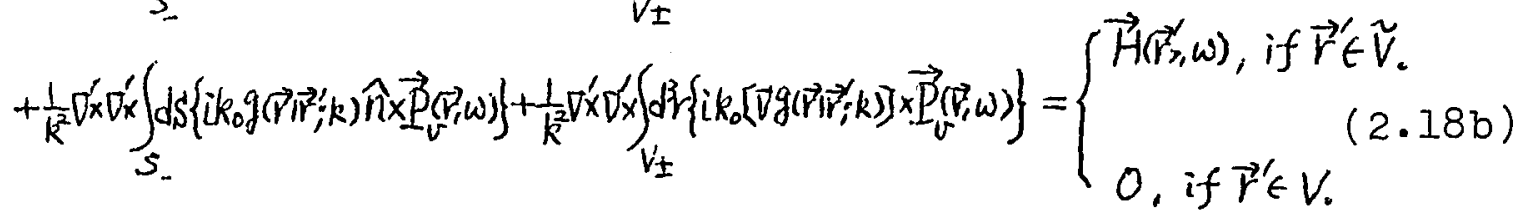

The incident waves with the wave number $k$ in the medium $M_{0}$ are,

and

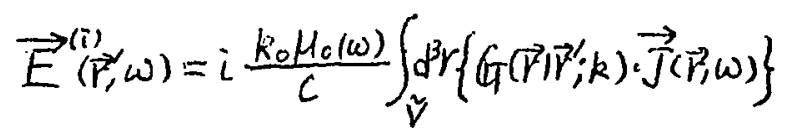

$$
\vec{H}^{(i)}\left(\vec{r}^{\prime}, \omega\right)=\int_{\vec{V}} d^{3} r\left\{G\left(\vec{r} \mid \vec{r}^{\prime} ; k\right) \cdot(\nabla \times \vec{J}(\vec{r}, \omega))\right\} .
$$

When the observation point $\vec{r}^{\prime}$ is in $V$, the formal solutions, Eqs. (2.18a) and (2.18b), express the new form of the extinction theorem. These solutions will reduce to Eqs. (2.14) and (2.15), if the medium $\mathbb{M}_{0}$ is replaced by vacuum $\left(\mu_{0}(\omega)=\epsilon_{0}(\omega)=1\right)$.

4. A SIMPIE APPLICATION OF THE EXTINCTION THEOREM AND THE IORENTZ-LORENZ FORMULA.

In this section, we first look for a formal solution of the macroscopic Maxwell's wave equations, which contain a "local effective field". We then use the extinction theorem to express the local effective field in terms of mean fields. We also use this expression to obtain the LorentzLorenz formula.

Let us assume that the medium $\mathrm{M}_{0}$ is a vacuum where the source of incident radiation is located, and that the 
radiation enters into the material $\mathbb{M}_{1}$ through the transition region $V_{ \pm}$. The medium $M_{1}$ is assumed to be a linear, isotropic, homogeneous, absorbing and non-magnetic dielectric. When an electromagnetic wave is incident on the dielectric, it induces microscopic charges, currents, etc., inside the dielectric.

By the assumption, $\vec{j}(\vec{r}, \omega)=\vec{M}(\vec{r}, \omega)=0$ in $\mathbb{I}_{1}$, the macroscopic Maxwell wave equation in the entire region $\widetilde{V}+V_{ \pm}+V$, can be written as

$$
\nabla \times[\nabla \times \vec{E}(\vec{r}, \omega)]-\frac{\omega^{2}}{c^{2}} \vec{E}(\vec{r}, \omega)=\frac{4 \pi}{c^{2}} \omega^{2} \vec{P}(\vec{r}, \omega)+4 \pi i \frac{\omega}{c^{2}} \vec{J}(\vec{r}, \omega) .
$$

Using Eq. (2.4a), we again look for a formal solution of Eq. (2.19). In following the method used to derive Eq. (2.6), we now carry the integration over the entire region, $\tilde{\mathrm{V}}+\mathrm{V}_{ \pm}+\mathrm{V}$, to obtain

$$
\begin{aligned}
\vec{E}\left(\vec{r}^{\prime}, \omega\right)= & \frac{i k_{0}}{C} \int_{\vec{V}_{\ldots} r} d \tau\left(\vec{r}\left(\vec{r}^{\prime} ; k_{0}\right) \cdot \vec{J}(\vec{r}, \omega)+\vec{R}_{0}^{2} \int_{V_{ \pm}+V} d^{3} r\left(T\left(\vec{r} \mid \vec{r}^{\prime} ; k_{0}\right) \cdot \vec{P}(\vec{r}, \omega)\right.\right. \\
& +\frac{1}{4 \pi} \int_{\vec{V}+V_{ \pm}+V} d^{3} r\{[\nabla \times(\nabla \times(T)) \cdot \vec{E}(\vec{r}, \omega)-G(T \cdot[\nabla \times(\nabla \times \vec{E})]\} .
\end{aligned}
$$

Term I represents the incident electric field $\overrightarrow{\mathrm{E}}^{(i)}\left(\vec{r}^{\prime}, \omega\right)$. Term II represents the total scattered field at $\vec{r}^{\prime}$ due to all the induced dipoles in the region $V_{+} V_{ \pm}$. Since term III can be expressed in the form of a surface intergral by Gauss' theorem, it vanishes upon integration over all space. Therefore, we find the mean electric field for $\vec{r}^{\prime} \in V$ to be

$$
\vec{E}\left(\vec{r}_{<}^{\prime}, \omega\right)=\vec{E}^{(\prime \prime}\left(\vec{V}_{i}^{\prime} \omega\right)+k_{0}^{2} \int_{V_{ \pm}+V}^{d{ }^{3} r}\left\{\left\{\left(\vec{r} \mid \vec{V}_{<}^{\prime} ; k_{0}\right) \cdot \vec{P}(\vec{P}, \omega)\right\} .\right.
$$


The local effective field, polarizing a molecule at $\vec{r}_{<}^{\prime}$, can be obtained by removing the polarized molecule at $\vec{r}_{<}^{\prime}$ from the above volume integral. The effective field is then found to be

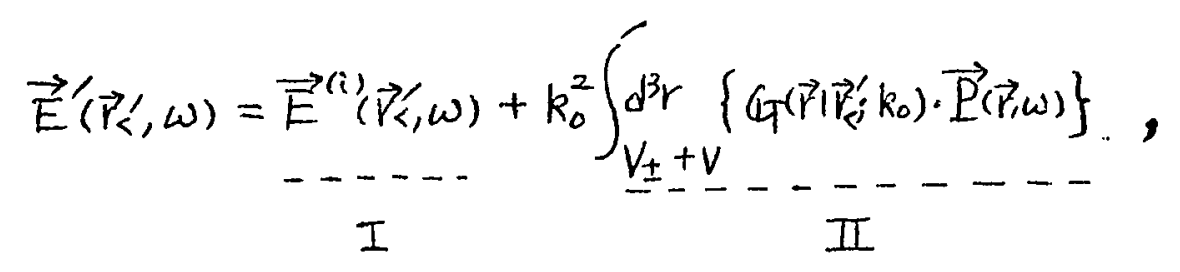

where the prime " $"$ on the integral represents the exclusion of a small volume in the neighborhood of $\vec{r}_{<}^{\prime}$. Term II in Eq. (2.22) thus expresses the total scattered field at $\vec{r}_{<}^{\prime}$ due to all the induced dipoles in $M_{1}$ except for the dipole at $\vec{r}_{<}^{\prime}$.

We now use the extinction theorem to simplify term II in Eq. (2.22). Using Eq. (2.5), we first rewrite it as

$$
\text { II }=\int_{V_{ \pm}+V}^{\prime} d^{3} r\left\{\nabla^{\prime} \times\left[\nabla^{\prime} \times g\left(\vec{r}, \vec{r}_{<}^{\prime} ; k_{0}\right) \overrightarrow{\mathrm{E}}(\vec{r}, \omega)\right]\right\} .
$$

We then use the following well-known mathematical identity,

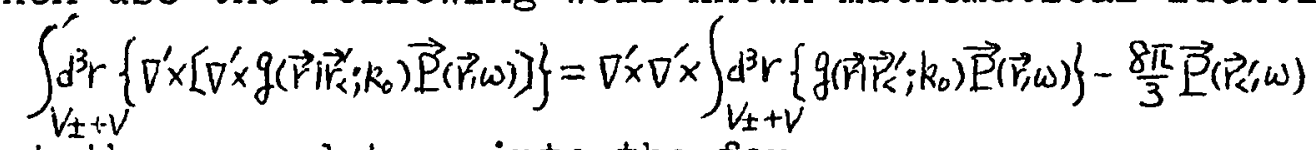

to get the second term into the form;

$$
\text { II }=\nabla^{\prime} \times \nabla^{\prime} \times \int_{V_{ \pm}} d^{3} r\left\{g\left(\vec{r} \vec{k}_{<}^{\prime} ; k_{0}\right) \vec{P}(\vec{r}, \omega)\right\}+\nabla^{\prime} \times \nabla^{\prime} \times \int_{V} d^{r}\left\{\left\{g\left(\vec{r} \mid \vec{r}_{<}^{\prime} ; k_{0}\right) \overrightarrow{\mathbb{P}}(\vec{r}, \omega)\right\}-\frac{8 \pi}{3} \vec{P}\left(\vec{r}_{<}^{\prime}, \omega\right)\right. \text {. }
$$

In order to express the second term on the right side of Eq. (2.24) in terms of a surface integral, we use the following set of wave equations for the bulk region $V$;

$$
\nabla^{2} \vec{P}(\vec{r}, \omega)+R_{0}^{2} \in(\omega) \vec{P}(\vec{r}, \omega)=0 \text {, }
$$

where $\epsilon(\omega)$ is the dielectric constant for the bulk material 
$M_{1}$, and

$$
\nabla^{2} g\left(\vec{r} \mid \vec{r}^{\prime} ; k_{0}\right)+k_{0}^{2} g\left(\vec{r} \mid \vec{r}^{\prime} ; k_{0}\right)=-4 \pi \delta\left(\vec{r}-\vec{r}^{\prime}\right) .
$$

From these two equations, we obtain for a point $\vec{r}_{<}^{\prime}$ in $V$,

$$
\int_{V} d^{3} r\left\{g\left(\vec{r} \vec{r}^{\prime} ; k_{0}\right) \vec{P}(\vec{r}, \omega)\right\}=\frac{4 \pi}{k_{0}^{2}(\epsilon(\omega)-1)} \vec{P}\left(\vec{r}_{<}^{\prime}, \omega\right)-\frac{1}{k_{0}^{2}(-(\omega)-1)} \int_{S_{-}} d s\left\{g\left(\vec{r} \mid \vec{r}^{\prime} ; k_{0}\right) \frac{\partial}{\partial n} \vec{P}(\vec{r}, \omega)-\vec{P}(\vec{r}, \omega) \frac{\partial}{\partial n} g\left(\vec{r} \vec{k}^{\prime} ; k_{0}\right)\right\} .
$$

First inserting Eq. (2.26) into Eq. (2.24) and then assuming that $\vec{P}\left(\vec{r}^{\prime}, \omega\right)$ is transverse, i.e.,

$$
\nabla^{\prime} \times\left(\nabla^{\prime} \times \vec{P}\left(\vec{r}_{<}^{\prime}, \omega\right)\right)=k_{0}^{2} \in(\omega) \vec{P}\left(\vec{r}_{<}^{\prime}, \omega\right)
$$

with the relation,

$$
\vec{P}\left(\vec{r}_{<}, \omega\right)=\frac{\epsilon(\omega)-1}{4 \pi} \vec{E}\left(\vec{r}_{<}^{\prime}, \omega\right) \text {. }
$$

Eq. (2.24) can be put in a final form,

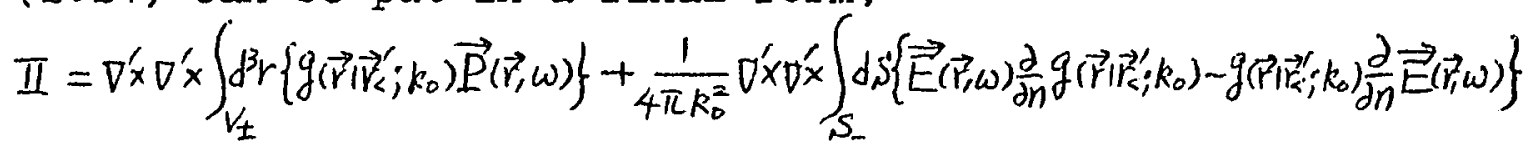

$$
\begin{aligned}
& +\vec{E}\left(\vec{r}^{\prime}, \omega\right)+\frac{4 \pi}{3} \vec{P}\left(\vec{r}^{\prime} \omega\right) \text {. }
\end{aligned}
$$

Here the extinction theorem, Eq. (2.14), requires that the first two terms must be extinguished everywhere in $V$, by the incident wave, $\vec{E}^{(i)}(\vec{r}, \omega)$. The extinction theorem also claims that the first two terms in Eq. (2.28) must represent the waves which propagate with the vacuum phase velocity. Thus, the effective field will satisfy the well known equation:

$$
\vec{E}^{\prime}\left(\vec{r}_{<}^{\prime}, \omega\right)=\vec{E}\left(\vec{r}_{<}^{\prime}, \omega\right)+\frac{4 \pi}{3} \vec{P}\left(\vec{r}_{<}^{\prime}, \omega\right) \quad\left(\vec{R}_{<}^{\prime} \in V\right) .
$$

We now derive the Lorentz-Lorenz formula. To do this we note that it is the effective field which polarizes the molecule at $\vec{r}_{<}^{\prime}$. Assuming a linear relationship, the induced 
dipole moment is given by

$$
\vec{p}\left(\vec{r}^{\prime}, \omega\right)=\alpha^{\prime}(\omega) \vec{E}^{\prime}\left(\vec{p}^{\prime} \omega\right),
$$

where $\alpha^{\prime}(\omega)$ is the molecular polarizability. Using the mean polarizability, $\alpha(\omega)$, we obtain the macroscopic polarization field,

$$
\vec{P}\left(\vec{r}^{\prime}, \omega\right)=N \alpha(\omega) \vec{E}^{\prime}\left(\vec{r}^{\prime}, \omega\right)
$$

where $N$ is the number of dipoles per unit volume. In the transition region of $\mathbb{M}_{1}, N$ may be a function of the position $\vec{r}$, but it is constant in the region V. Inserting Eq. (2.29) into $\mathrm{Eq} \cdot(2 \cdot 30)$, we obtain

$$
\vec{P}\left(\vec{r}^{\prime}, \omega\right)=N \alpha(\omega)\left[\vec{E}\left(\vec{P}^{\prime}, \omega\right)+\frac{4 \pi}{3} \vec{E}\left(\vec{r}^{\prime}<, \omega\right)\right] \quad\left(\vec{r}^{\prime}<V\right) .
$$

Using the relation of Eq. (2.27), this leads to

$$
\left[1-\frac{\epsilon(\omega)+2}{\epsilon(\omega)-1} \frac{4 \pi}{3} N \alpha(\omega)\right] \vec{P}\left(\vec{r}_{<}, \omega\right)=0
$$

so that we obtain the well known Lorentz-Lorenz formula,

$$
\frac{4 \pi}{3} N \alpha(\omega)=\frac{\epsilon(\omega)-1}{\epsilon(\omega)+2}
$$

We now note that if the absorption in the dielectric $M_{1}$ is so strong that the field quantities, $\vec{E}$ and $\vec{P}$, of Eq. (2.28) virtually vanish at the surface $S_{-}$(also in the bulk region $\mathrm{V}$ ), the formal solution of $\mathrm{Eq} .(2.22)$ leads to

$$
0=\vec{E}^{(i)}\left(\vec{r}^{\prime}, \omega\right)+\nabla^{\prime} \times \nabla^{\prime} \times \int_{V_{ \pm}} d^{3} r\left\{g\left(\vec{r} \mid \vec{r}^{\prime} ; k_{0}\right) \vec{P}(\vec{r}, \omega)\right\} \quad\left(\vec{r}^{\prime} \in V\right) .(2.33)
$$

For the case where the vacuum is replaced with the medium $M_{0}$ of dielectric constant $\epsilon_{0}(\omega)$, as in section 3 , we can follow the analysis above. To do this, we first note that the virtual polarization is related to the mean electric 
field through the following relation,

$$
\vec{P}_{v}\left(\vec{r}_{<}^{\prime}, \omega\right)=\eta_{v}(\omega) \vec{E}\left(\vec{r}_{<}^{\prime}, \omega\right)
$$

with the virtual electric susceptibility, $\eta_{v}(\omega)$, defined as

$$
\eta_{v}(\omega)=\frac{1}{4 \pi}\left(\epsilon(\omega)-\epsilon_{0}(\omega)\right)
$$

and in terms of the effective electric field,

$$
\vec{P}_{v}\left(\vec{r}_{k}^{\prime}, \omega\right)=N \alpha_{v}(\omega) \vec{E}^{\prime}\left(\vec{r}_{s}^{\prime}, \omega\right) \text {, }
$$

where $\alpha_{v}(\omega)$ is the virtual mean polarizability. We then obtain expressions similar to Eqs. (2.29), (2.32b) and $(2.33):$

$$
\begin{aligned}
& \overrightarrow{\vec{E}^{\prime}}\left(\vec{r}_{<}^{\prime}, \omega\right)=\vec{E}\left(\vec{r}_{<}^{\prime}, \omega\right)+\frac{4 \pi}{3} \frac{\vec{P}_{v}\left(\vec{r}_{<}^{\prime}, \omega\right)}{\epsilon_{0}(\omega)}, \\
& \frac{4 \pi}{3} N \frac{\alpha_{v}(\omega)}{\epsilon_{0}(\omega)}=\frac{\epsilon(\omega)-\epsilon_{0}(\omega)}{\epsilon(\omega)+2 \epsilon_{0}(\omega)},
\end{aligned}
$$

and

$$
0=\vec{E}(\stackrel{i}{(\vec{k})}, \omega)+\frac{k_{0}^{2}}{k^{2}} \nabla^{\prime} \nabla^{\prime} \times \int_{V_{ \pm}} d^{3} r\left\{g\left(\vec{r} \mid \vec{r}_{<}^{\prime} ; k\right){\overrightarrow{E_{v}}}_{v}(\vec{r}, \omega)\right\} \quad\left(k=k_{0} \sqrt{\epsilon_{0}(\omega)}\right) .
$$

5. SUMMARY OF THE GENERALIZED EXTINCTION THEOREM

Eqs. (2.18a) and (2.18b) formally express the generalized extinction theorem, if the observation point $\vec{r}^{\prime}$ belongs to the bulk material region of $\mathrm{M}_{1}$. Our results are more general than those which have appeared in the literature. The boundary used is physical, not necessarily geometrically sharp and the medium $M_{0}$ is not necessarily a vacuum. If we must consider a strong surface effect, such modifications become important. When the entire space is filled with a medium $\mathbb{M}_{0}$ 
without $\mathbb{M}_{1}$, the virtually induced fields, $\vec{P}_{v}$ and $\vec{M}_{v}$, in Eqs. $(2.18 \mathrm{a})$ and $(2.18 \mathrm{~b})$ are zero. Then the extinction theorem can be read as the mathematical statement of Huygens' principle for the propagation of the incident waves (see also J. J. Sein, 1970). On the other hand, if $M_{1}$ is present somewhere in space $\left(\vec{P}_{v} \neq 0, \vec{M}_{v} \neq 0\right)$, the reflection and refraction of the incident waves occur at the boundary of $\mathbb{M}_{1}$. Then the extinction theorem does not reflect Huygens' principle for the propagation of the refracted wave, instead it describes the propagation of the incident waves which enter the medium $\mathrm{M}_{1}$ without modification of phase, phase velocity, amplitude or propagation direction. The propagation of the incident wave in $\mathbb{M}_{1}$ is virtual, since it will be extinguished everywhere in the bulk region of $M_{1}$ by the virtual scattered wave which is a part of the scattered wave due to $\vec{P}_{v}$ and $\vec{M}_{v}$. The extinction of the incident wave can be looked upon as the destructive interference between the incident wave and a part of the scattered wave in the material $\mathbb{M}_{1}$. Then a definite phase relation between the two waves should be required. The precise analysis of the phase relation becomes possible when we remove a locality in space from our formal solutions by expressing them in terms of plane wave expansions. This will be discussed in the next chapter. 


\section{CHAPTER III}

\section{THE EXTINCTION THEOREM IN ANOMALOUS DISPERSION WITH PLANE BOUNDARIES}

\section{INTRODUCTION}

In this chapter, we will treat anomalous dispersion. For this purpose, let us closely examine the role of the generalized extinction theorem as a boundary condition in order to determine the unique response of a simple material to an incident radiation field. The extinction of the incident wave everywhere in the bulk region of the material medium can be looked upon as a result of the destructive interference with a part of the induced wave. To find the precise phase relation for the interference, we need an expression of such form that the phases can be defined at every observation point in the material medium. We thereby assume that every harmonic field quantity involved can be expressed as a linear superposition of plane waves (inhomogeneous plane waves if absorption is involved), propagating in all directions in space. This linear superposition of plane waves is known as a "plane wave mode expansion". Such a mode expansion has been successfully applied by E. Lalor and E. Wolf (1972), in deriving Fresnel's law at the plane interface between a vacuum and a linear, homogeneous, isotropic, non-magnetic and non-absorbing dielectric filling a 
semi-infinite space. However, in their analysis, anomalous dispersion was not treated.

Since the refractive index is complex in the frequency region of anomalous dispersion, the plane wave mode expansions of the field quantities in the absorbing material must be obtained. Using these expansions, we will investigate optical effects of two different surface boundaries: the physical boundary, which will be discussed further in Chapter IV, V, and VI, and the geometrically sharp boundary.

\section{FULLY NON-IOCAL REPRESENTATIONS}

(IN SPACE AND TIME) OF FORMAL SOLUTIONS FOR MAXWELI'S WAVE EQUATIONS

Let us assume that the media $\mathbb{M}_{0}$ and $\mathbb{M}_{1}$ are isotropic, homogeneous, linear and non-magnetic. $\mathbb{M}_{1}$ is absorbing and occupies the half space $Z>0$ with $M_{0}$ occupying the region $Z<0$. Therefore the surface $S+$ where the transition region $V_{ \pm}$starts is taken to be the $x-y$ plane, $z=0$. Since $\vec{j}(\vec{r}, \omega)=\vec{M}_{w}(\vec{r}, \omega)=0$ and $\mu_{0}(\omega)=1$ in the present case, Eqs. $(2.18 a)$ and $(2.18 b)$ lead to,

$$
\begin{aligned}
& \vec{E}^{(i)}\left(\vec{r}^{\prime}, \omega\right)+\frac{1}{4 \pi k^{2}} \nabla^{\prime} \nabla^{\prime} \times \int_{S_{-}} d S\left\{\vec{E}(\vec{r}, \omega) \frac{\partial}{\partial n} g\left(\vec{r} \vec{r}^{\prime} ; k\right)-g\left(\vec{r} \vec{r}^{\prime} ; k\right) \frac{\partial}{\partial n} \vec{E}(\vec{r}, \omega)\right\}-\frac{1}{k^{2}} \nabla^{\prime} \times \nabla^{\prime} \int_{S_{-}} d S\left\{g\left(\vec{r} \vec{r}^{\prime} ; k\right) \hat{n} \nabla \cdot \vec{P}(\vec{r}, \omega)\right\} \\
& +\frac{1}{k^{2}} \nabla^{\prime} \times \nabla^{\prime} \iint_{V_{ \pm}} d^{3} r\left\{k_{0}^{2} g\left(\vec{r} \mid \vec{r}^{\prime} ; k\right) \vec{P}_{v}(\vec{r}, \omega)\right\}=\left\{\begin{array}{c}
\vec{E}\left(\vec{r}^{\prime}, \omega\right), \text { if } \vec{r}^{\prime} \in \vec{V}, \\
0, \text { if } \vec{r}^{\prime} \in V,
\end{array}\right.
\end{aligned}
$$

and

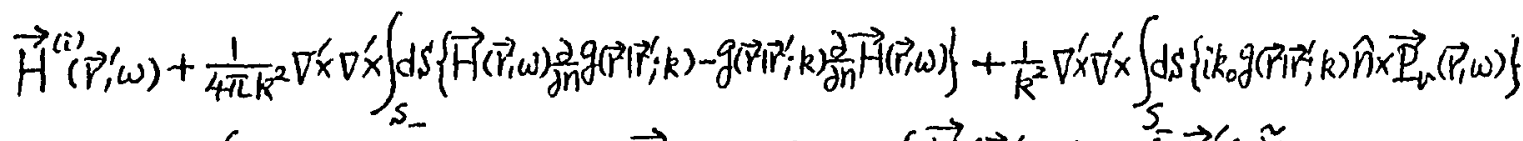

$$
\begin{aligned}
& +\frac{1}{k^{2}} \nabla^{\prime} \times \nabla^{\prime} \int_{V_{ \pm}} d^{3}\left\{\left\{i k_{0}\left[\nabla g\left(\vec{r}, \vec{r}^{\prime} ; k\right)\right] \times \overrightarrow{\mathrm{P}}_{w}(\vec{r}, \omega)\right\}=\left\{\begin{array}{c}
\vec{H}\left(\vec{r}^{\prime}, \omega\right), \text { if } \vec{r}^{\prime} \in \tilde{V}, \\
0, \text { if } \vec{r}^{\prime} \in V_{0}(3.1 b)
\end{array}\right.\right. \\
& \text { We now consider anomalous dispersion. We can place }
\end{aligned}
$$


the surface $S_{-}$, defined in section 3 of Chapter II, at the plane $z=L$ where the radiation field virtually vanishes due to the strong resonance absorption of the dielectric. Then from Eq. (3.1a), the total electric and magnetic fields outside the medium $\mathbb{M}_{1}$ can be written as

and

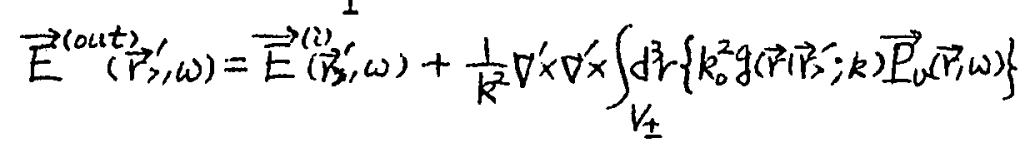

$$
\vec{H}^{(\text {out } t)}\left(\vec{r}^{\prime}, \omega\right)=\vec{H}^{(i)}\left(\vec{r}_{3}^{\prime}, \omega\right)+\frac{1}{k^{2}} \nabla^{\prime} \times \nabla^{\prime} \int_{V_{ \pm}} d \beta r\left\{i k_{0}\left[\nabla g\left(\vec{r} \mid \vec{r}^{\prime} ; k\right)\right] \times \vec{P}_{v}(\vec{r}, \omega)\right\} .
$$

Similarly the extinction theorems become, in $\mathrm{V}$,

$$
0=\vec{E}^{(i)}\left(\vec{r}_{<}^{\prime}, \omega\right)+\frac{1}{k^{2}} \nabla^{\prime} \times \nabla^{\prime} \times \int d_{V_{ \pm}} r\left\{\left\{k_{0}^{2} g\left(\vec{r} \mid \vec{r}^{\prime} ; k\right) \vec{P}_{v}(\vec{r}, \omega)\right\}\right.
$$

and

$$
0=\vec{H}^{\prime \prime \prime}\left(\vec{r}_{<}^{\prime}, \omega\right)+\frac{1}{k^{2}} \nabla^{\prime} \times \nabla^{\prime} \times \int_{V_{ \pm}} d^{3} r\left\{i k_{0}\left[\nabla g\left(\vec{r} \overrightarrow{r^{\prime}} ; k\right)\right] \times \vec{P}_{v}(\vec{r}, \omega)\right\} .
$$

We then introduce mode expansions, where each mode is a single plane wave with a specific vector amplitude (an expansion coefficient). Noting that the wave number in the medium $\mathbb{M}_{0}$ is $k=k_{0} n_{0}(\omega)$, the incident waves can be expressed in terms of their expansion coefficients, $\vec{e}^{(i)}(p, q, \omega)$ and $\vec{h}^{(i)}(p, q, \omega)$, as (see E. Lalor, 1968 and J. R. Shewell and E. Wolf, 1968),

$$
\begin{aligned}
& \vec{E}^{(i)}\left(\vec{r}^{\prime}, \omega\right)=\iint d p d q \vec{e}^{(i)}(p, q, \omega) \exp \left\{i k\left(p x^{\prime}+q y^{\prime}+m z^{\prime}\right)\right\}, \\
& \vec{H}^{(i)}\left(\vec{r}^{\prime}, \omega\right)=\iint^{\Delta} d p d q \vec{h}^{(i)}(p, q, \omega) \exp \left\{i k\left(p x^{\prime}+q y^{\prime}+m z^{\prime}\right)\right\},
\end{aligned}
$$

where the variables $p$ and $q$ are real and the integration domain, $p^{2}+q^{2} \leq 1$, is denoted by $\Delta$. In this domain, we have a real $\mathrm{m}$,

$$
m=+\sqrt{1-\left(p^{2}+q^{2}\right)}, \quad\left(p^{2}+q^{2} \leq 1\right)
$$


so that $p, q$, and $m$ represent the direction cosines of the propagation direction of the plane wave (i.e., mode). Each mode will be simply referred to by its direction cosines, $(p, q, m)$. We also express the total electric fields, $\vec{E}^{\text {(out) }}\left(\vec{r}^{\prime}, \omega\right)$, and the reflected field (the scattered field), $\vec{E}^{(r)}\left(\vec{r}_{>}^{\prime}, \omega\right)$, in terms of plane wave mode expansions,

$$
\vec{E}^{(\text {out })}(\vec{r}, \omega)=\iint d p d q \vec{e}^{(\text {out }}(p, q, \omega) \exp \left\{i k\left(p x^{\prime}+q y^{\prime}-m z^{\prime}\right)\right\}\left(z^{\prime}<0\right) \quad \text { (3.5a) }
$$

and

$$
\vec{E}^{(r)}\left(\vec{r}_{>}^{\prime}, w\right)=\iint d p d q \vec{e}^{(r)}(p, q, w) \exp \left\{i k\left(p x^{\prime}+q y^{\prime}-m z^{\prime}\right)\right\}\left(z^{\prime}<0\right)
$$

Similarly, for the magnetic fields, we have

and

$$
\vec{H}^{(\text {out })}\left(\vec{r}_{>1}^{\prime}(\omega)=\iint d p d q \vec{h}^{(\text {out })}(p, q, \omega) \exp \left\{i k\left(p x^{\prime}+q y^{\prime}-m z^{\prime}\right)\right\} \quad\left(z^{\prime}<0\right) \quad(3.6 \mathrm{a})\right.
$$

$$
\vec{H}^{(r)}\left(\vec{r}_{>}^{\prime}, \omega\right)=\iint d p d q \vec{h}^{(r)}(p, q, \omega) \exp \left\{i k\left(p x^{\prime}+q y^{\prime}-m z^{\prime}\right)\right\} \quad\left(z^{\prime}<0\right) \cdot(3.6 b)
$$

The outgoing Green's function $g\left(\vec{r} \mid \vec{r}^{\prime} ; k\right)$ can also be expressed as a bundle of plane waves propagating in all directions in space (Weyl, 1919, see also the text of J.A. Stratton, 1941),

$$
g\left(\vec{r} \mid \vec{r}^{\prime} ; k\right)=\frac{i k}{2 \pi} \iint_{-\infty}^{+\infty} d p d q \frac{1}{m} \exp \left\{i k\left(p\left(x^{\prime}-x\right)+q\left(y^{\prime}-y\right)+m\left|z-z^{\prime}\right|\right\}\right\} .
$$

We now assume that the incident wave in Eq. (3.2a) with real $\mathrm{m}$ induces fields in $\mathrm{M}_{1}$. The induced fields are in general composed of two parts: one is the wave which will be extinguished by the incident wave, whereas the other is the refracted wave. Let us first derive the mode expansions for the mean refracted electric field $\vec{E}(\vec{r}, \omega)$. For simplicity, we assume that $\vec{E}(\vec{r}, \omega)$ in the transition region, $V_{ \pm}$, obeys the wave equation:

$$
\nabla^{2} \vec{E}(\vec{r}, \omega)+k_{0}^{2} n^{2}(\omega) \vec{E}(\vec{r}, \omega)=0, \quad(z \geq 0)
$$


where $n(\omega)=\sqrt{\epsilon(\omega)}$ is the complex refractive index of $M_{1}$, $n(\omega)=n_{r}(\omega)+i n_{i}(\omega)$. We will see in Chapter $V$ that a surface effect in anomolous dispersion can be treated by such a simplified treatment. Any component of a solution of Eq. (3.8), $\vec{E}(\vec{r}, \omega)$, can be written in the form of

$$
E_{\alpha}(\vec{r}, \omega)=\lambda_{\alpha} X(x) Y(y) Z(z) \Omega(\omega), \quad(\alpha=x, y, \text { or } z),
$$

where the components satisfy

$$
\frac{d^{2} X}{d x^{2}}=-k_{0}^{2} n_{x}^{2} X, \frac{d^{2} Y}{d y^{2}}=-k_{0}^{2} n_{y}^{2} Y \text { and } \frac{d^{2} Z}{d z^{2}}=-k_{0}^{2} n_{z}^{2} Z, \quad\left(n^{2}=n_{x}^{2}+n_{y}^{2}+n_{z}^{2}\right)
$$

and $\lambda_{\alpha}$ is the $\alpha$ component of the polarization vector $\hat{\lambda}=\frac{\vec{E}(\vec{r}, \omega)}{|\vec{E}(\vec{\gamma}, \omega)|}$. The boundary is the $x-y$ plane at $z=0$ so that there is complete homogeneity in the $x-y$ plane. The plane of constant amplitude is then parallel to the $x-y$ plane, i.e., absorption occurs in the $z$ direction. Thus $n_{x}$ and $n_{y}$ are real, whereas $n_{z}$ is complex,

$$
n_{z}=n_{z}^{r}+i n_{z}^{i}
$$

Then the solution of Eq. (3.8) is given by

$$
E_{\alpha}(\vec{r}, \omega)=E_{\alpha}^{0} \exp \left\{i k_{0}\left(n_{x} \chi+n_{y} y+n_{z}^{r} z\right)-k_{0} n_{z}^{i} z\right\}, \quad(\alpha=x, y, \text { or } z) .
$$

We must note in Eq. (3.9) that the planes of constant phase and constant amplitude associated with a wave in an absorbing medium do not in general coincide. In order to avoid any possible confusion arising from this distinction, we define the propagation direction of the wave by the normal to the plane of constant phase. Then the vector $\vec{n}=\left(n_{x}, n_{y}, n_{z}^{r}\right)$ defines the propagation direction. In terms of spherical 
co-ordinates with the polar axis along the $\mathrm{z}$ direction, $n_{x}=|\vec{n}| \sin \theta^{\prime} \cos \phi^{\prime}, n_{y}=|\vec{n}| \sin \theta^{\prime} \sin \phi^{\prime}$ and $n_{x}^{r}=|\vec{n}| \cos \theta^{\prime}$, where $|\vec{n}|=\sqrt{n_{x}^{2}+n_{y}^{2}+n_{z}^{r^{2}}}$.

Since Eq. (3.8) is linear, any linear superposition of the plane waves, Eq. (3.9), of different $\vec{n}$, i.e., of different propagation directions, provided that $n(\omega)$ is constant, also satisfies Eq. (3.8). Thus, if the mean electric field $\vec{E}(\vec{r}, \omega)$ inside the absorbing medium is propagating in the positive $z$ direction, it can be expressed in terms of its expansion coefficient, $\vec{e}^{\prime}\left(\theta^{\prime}, \phi^{\prime}, \omega\right)$, as

$$
\vec{E}\left(\vec{r}_{2}, \omega\right)=\int d \Omega^{\prime} \vec{e}^{\prime}\left(\theta^{\prime}, \phi^{\prime}, \omega\right) \exp \left\{i k_{0}|\vec{n}|\left(\sin \theta^{\prime} \cos \phi^{\prime} x+\sin \theta^{\prime} \sin \phi^{\prime} y+\cos \theta^{\prime} z\right) k_{0} n_{z}^{i} z\right\}
$$

where $d \Omega^{\prime}=\sin \theta^{\prime} d \theta^{\prime} d \phi^{\prime}$.

Let us now change the variables of the integration, from the polar angles $\left(\theta^{\prime}, \phi^{\prime}\right)$ to the direction cosines $\left(p^{\prime}, q^{\prime}\right)$ defined by

$$
p^{\prime}=\sin \theta^{\prime} \cos \phi^{\prime}, q^{\prime}=\sin \theta^{\prime} \sin \phi^{\prime} \text { and } m^{\prime}=\cos \theta^{\prime} \text {. }
$$

Here $\mathrm{m}^{\prime}$ can also be written as

$$
m^{\prime}=\sqrt{1-\left(p^{\prime 2}+q^{\prime 2}\right)} \text {. }
$$

If $\mathrm{p}^{\prime}, \mathrm{q}^{\prime}$ and $\mathrm{m}^{\prime}$ are real, they represent the direction cosines of the propagating wave (the refracted mode). They are not arbitrary, for the refracted mode depends on the incident mode. We may also have a situation of total internal reflection in which $\mathrm{m}^{\prime}$ is purely imaginary. In order to extend our expression above to include such a possibility, we choose $p^{\prime}$ and $q^{\prime}$ to be real and allow them to vary from positive to 
negative infinity. Thus we can write Eq. (3.11) as

$\left.\vec{E}(\vec{k}, \omega)=\iint_{-\infty}^{\infty} d p d q^{\prime} \vec{e}\left(p^{\prime}, q^{\prime}, \omega\right) \exp \left\{i k_{0} \mid \vec{n}\right)\left(p^{\prime} x+q^{\prime} y+m^{\prime} z\right)-k_{0} n_{z}^{i} z\right\},(z \geq 0)$.

Since the virtual polarization field is linearly re-

lated to $\vec{E}\left(\vec{r}_{r}, \omega\right)$ through Eq. (2.34a), we express the virtual field in terms of its expansion coefficient, $\vec{Q}\left(p^{\prime}, q^{\prime}, \omega\right)$ as

$$
\vec{P}_{v}(\vec{r}<, \omega)=\iint_{-\infty}^{+\infty} d p^{\prime} d q^{\prime} \vec{Q}\left(p^{\prime}, q^{\prime}, \omega\right) \exp \left\{i k_{0}|\vec{n}|\left(p^{\prime} x+q^{\prime} y+m^{\prime} z\right)-k n_{z}^{i} z\right\}(z \geq 0) .
$$

The magnetic field in $M_{1}$ obeys the equation similar to Eq. (3.8) so that we can also express it in terms of the plane wave mode expansion,

$$
\vec{H}(\vec{r}, \omega)=\int_{-\infty}^{+\infty} \int d p^{\prime} d q^{\prime} \vec{h}\left(p, q^{\prime}, \omega\right) \exp \left\{i k_{0}|\vec{h}|\left(p x+q^{\prime} y+m^{\prime} z\right)-k_{0} n_{z}^{i} z\right\} \quad(z \geq 0) .
$$

In order to apply the extinction theorem, we first evaluate the volume integral of Eq. (3.3a) using Eqs. (3.7) and $(3.14)$,

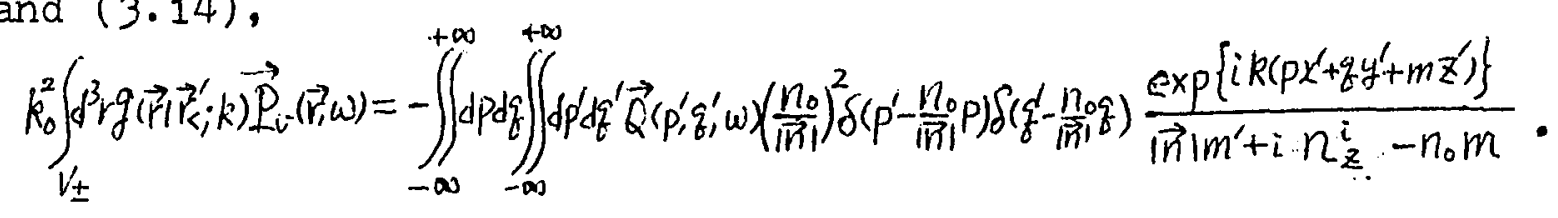

In this integral, the Dirac delta functions lead to Snell's law (see Eq. (3.18)). We also note that waves are propagating with the phase velocities of the medium $\mathbb{M}_{0}$. We can then write Eq. (3.3a) as

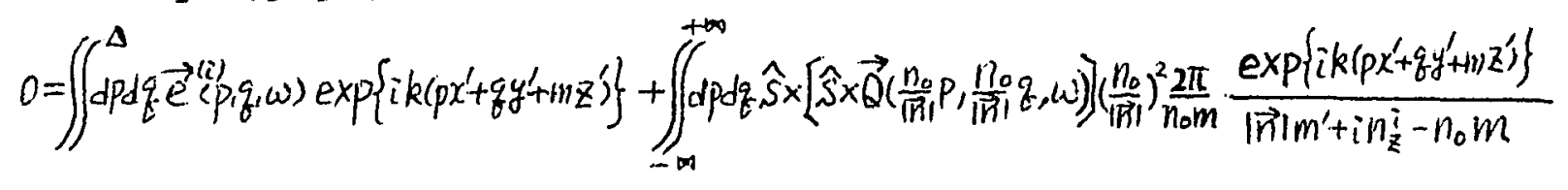

where the integration domain, $\Delta$, is referred to the region $p^{2}+q^{2} \leq 1$ and the unit vector $\hat{S}$ is $(p, q, m)$. Here, it is also required by the extinction of the incident plane waves every where in the region. $V$, that the induced plane waves must 
propagate with the same phase velocities as those of the incident plane waves. We thus obtain

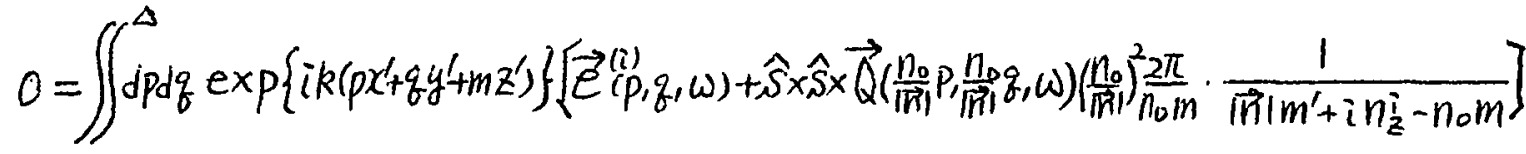

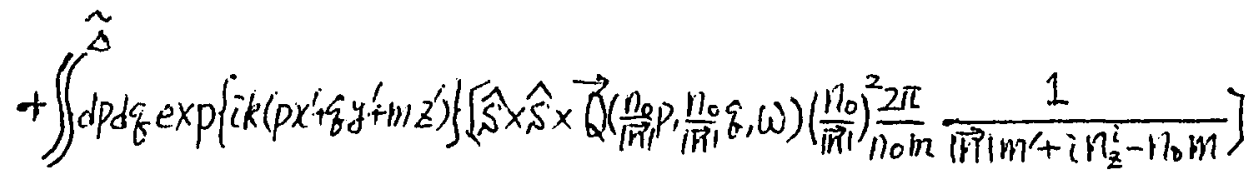

where the integration domain, $\widetilde{\Delta}$, is referred to as $\mathrm{p}^{2}+\mathrm{q}^{2}>1$. Since the expressions of $\exp \left\{i k\left(p x^{\prime}+q y^{\prime}+m z^{\prime}\right)\right\}$ for each different mode are linearly independent, the amplitudes must satisfy, for $p^{2}+q^{2} \leq 1$,

$$
0=\vec{e}^{(i)}(p, q, \omega)+\hat{S} \times\left[\hat{S} \times \vec{Q}\left(\frac{n_{0}}{\vec{n} \mid} p, \frac{n_{0}}{\overrightarrow{\vec{n}} q}, \omega\right)\left(\frac{n_{0}}{\vec{n}\left|{ }^{2}\right|}\right)^{2}\right] \frac{2 \pi}{n_{0} m} \frac{1}{|\vec{n}| m^{\prime}+i n_{z}^{i}-n_{0} m},
$$
whereas, for $p^{2}+q^{2}>1$,

$$
\vec{Q}\left(\frac{n_{0}}{\vec{n} \mid} p, \frac{n_{0}}{|\vec{n}|} q, \omega\right)=0 \text {. }
$$

Eq. (3.17a) expresses the spatially non-local form of the extinction theorem. Here we also recall that because of the delta functions, $\delta\left(p^{\prime}-\frac{n_{0}}{|\vec{n}|} p\right)$ and $\delta\left(q^{\prime}-\frac{n_{0}}{\mid \vec{w}^{\prime}} q\right)$, the incident mode $(p, q, m)$ is uniquely related to the refracted mode $\left(p^{\prime}, q^{\prime}, m^{\prime}\right)$ in $\vec{Q}\left(p^{\prime}, q^{\prime}, \omega\right)$ through the following relations;

$$
p^{\prime}=\frac{n_{0}}{|\vec{n}|} p, q^{\prime}=\frac{n_{0}}{|\vec{n}|} q \text { and } m^{\prime}=\sqrt{1-\left(\frac{n_{0}}{\vec{n} \mid}\right)^{2}\left(p^{2}+q^{2}\right)} \text {. }
$$

These are just Snell's law (see the next section). We can now express Eq. (3.2a) in terms of the mode expansions. Noting that the second term of Eq. (3.2a) is the reflected field, $\vec{E}^{(r)}\left(\vec{r}_{>}^{\prime}, w\right)$, we obtain, for the incident 
mode $(p, q, m)$ satisfying $p^{2}+q^{2} \leq 1$,

$$
\vec{e}^{(\text {out })}(p, q, \omega)=\vec{e}^{(i)}(p, q, \omega)+\vec{e}^{(r)}(p, q, \omega) \text {, }
$$

with

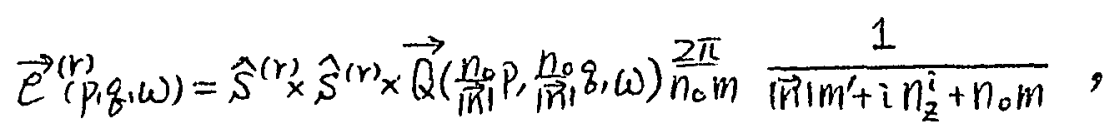

where the real unit vector $\hat{S}^{(r)}$ is the reflected mode $(p, q,-m)$. In order to find the relationship between $\vec{e}\left(p^{\prime}, q^{\prime}, \omega\right)$ of the refracted field and $\vec{Q}\left(p^{\prime}, q^{\prime}, \omega\right)$ of the virtual polarization, we use the definition of the virtual polarization, Eqs. (2.34a) and (2.34b). We then obtain the relation,

$$
\vec{Q}\left(p^{\prime}, q^{\prime}, \omega\right)=\frac{1}{4 \pi}\left(n^{2}(\omega)-n_{c}^{2}(\omega)\right) \vec{e}\left(p^{\prime}, q^{\prime}, \omega\right) \text {. }
$$

Using this relation, we can express Eqs. (3.17a) and (3.19b) in terms of the vector amplitude of the mean electric field. They are

$$
\vec{e}_{(p, q, \omega)}^{(i)}+\frac{1}{2} \hat{s} \times \hat{s} \times \vec{e}\left(\frac{n_{0}}{|\vec{m}|} p_{1}, \frac{n_{0}}{\mid \vec{n} i}, q_{,} \omega\right)\left(\frac{n_{0}}{|\vec{n}|}\right)^{2} \frac{n^{2}-n_{0}^{2}}{n_{0} m\left(|\vec{n}| m^{\prime}+i n_{z}^{i}-n_{0} m\right)}=0
$$

and

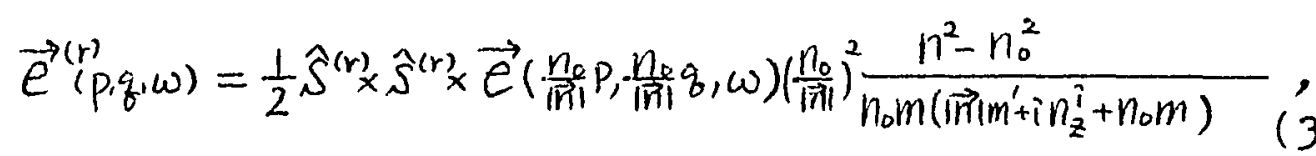

where $p^{2}+q^{2} \leq 1$

We now derive similar expressions for the magnetic

field. After calculations similar to those for the electric fields, Eqs. (3.2b) and $(3.3 b)$ can be written as, for $p^{2}+q^{2} \leq 1$,

$$
\vec{h}^{(o u(t)}(p, q, \omega)=\vec{h}^{(i)}(p, q, \omega)+\vec{h}^{(r)}(p, q, \omega)
$$

and 
where

$$
\left.0=\vec{h}_{(p, q}^{(i)}, \omega\right)+\hat{S} \times \hat{S} \times\left[\hat{S} \times \vec{Q}\left(\frac{n_{0}}{\mid \vec{n}} p, \frac{n_{0}}{|\vec{n}| q}, \omega\right)\left(\frac{n_{0}}{|\vec{n}|}\right)^{2}\right] \frac{2 \pi}{m\left(\vec{n} \mid m^{\prime}+i n_{z}^{i}-n_{0} m\right)},
$$

$$
\vec{h}^{(r)}=\hat{S}^{(r)} \times \hat{S}^{(r)} \times\left[\hat{S}^{(r)} \times \vec{Q}\left(\frac{n_{0} p}{|\vec{n}|}, \frac{n_{0}}{|\vec{n}|} q, \omega\right)\left(\frac{n_{0}}{|\vec{n}|}\right)^{2} \frac{2 \pi}{m\left(|\vec{n}| m^{\prime}+i n_{z}^{i}+n_{0} m\right)}\right. \text {. }
$$

For later convenience, we relate $[\hat{S} \times \vec{Q}]$ of Eq. (3.22b) and $\left[\hat{S}^{(r)} \times \vec{Q}\right]$ of Eq. (3.22c) to the magnetic vector amplitude, i.e., the expansion coefficient, $\vec{h}$, defined in Eq. (3.15). In order to do this, let us consider Maxwell's equation in $\mathrm{V} \pm$,

$$
\nabla \times \vec{H}(\vec{r}, \omega)=-i k_{0} n^{2}(\omega) \vec{E}(\vec{r}, \omega)
$$

Here we recall that $\mu_{0}(\omega)=\mu(\omega)=1$ and $n(\omega)$ is the complex refractive index, $\boldsymbol{n}(\omega)=\sqrt{\epsilon(\omega)}$. Then, substituting the plane wave mode expansions of Eq. (3.13) and (3.15) into Eq. (3.23), we obtain,

$$
i k_{0}\left(|\vec{n}| \hat{s}^{\prime}+i n_{z}^{i} \hat{z}\right) \times \vec{h}\left(p^{\prime} q^{\prime}, \omega\right)=-i k_{0} n^{2} \vec{e}\left(p^{\prime} q^{\prime}, \omega\right),
$$

where the unit vector $\hat{S}^{\prime}$ is $\left(p^{\prime}, q^{\prime}, m^{\prime}\right)$. Taking the vector product of the unit vector $\hat{S}=(p, q, m)$ and Eq. (3.24), then using Eq. (3.20), we obtain

$$
\left.-\left[|\vec{n}|\left(\hat{s} \cdot \hat{s}^{\prime}\right)+i n_{z}^{i}(\hat{s} \cdot \hat{z})\right] \vec{h}+\mid \vec{n}\right)(\hat{s} \cdot \vec{h}) \hat{S}^{\prime}+i n_{z}^{i}(\hat{s} \cdot \vec{h}) \hat{z}=-n^{2} \frac{4 \pi}{n^{2}-n_{0}^{2}} \hat{s} \times \vec{Q}\left(p^{\prime} \hat{q}^{\prime}, \omega\right) .
$$

We can obtain similar results for the expression, $\left[\hat{S}^{(r)} \times \vec{Q}\right]$, if we replace $\hat{S}^{\prime}$ with $\hat{S}^{(r)}$.

In the next section, we use the precise phase relations among the various vector amplitudes obtained here as basic equations to derive Fresnel's formula. We also note that these basic equations are fully non-local representations 
(in space) of our formal solutions for anomalous dispersion.

3. A NEW DERIVATION OF SNELL'S LAW AND FRESNEL'S FORMULA

The extinction of the incident wave everywhere in the bulk medium $M_{1}$ (in $V$ ), requires that the polarization field in $V_{ \pm}$must produce an induced wave (the scattered wave) in $V$, a part of which propagates with the same phase velocity and propagation direction as that of incident wave, but with the opposite phase (see Eqs. (3.16) and (3.17a)). We then obtain the relationship between the incident and the refracted mode to be (see Eq. (3.18)),

$$
p^{\prime}=\frac{n_{0}}{|\vec{n}|} p, q^{\prime}=\frac{n_{0}}{|\vec{n}|} q, m^{\prime}=\sqrt{1-\left(\frac{n_{0}}{|\vec{n}|}\right)^{2}\left(p^{2}+q^{2}\right)} \text { and } m=\sqrt{1-\left(p^{2}+q^{2}\right)} \text {, }
$$

where $m$ is real because of $p^{2}+q^{2} \leq 1$. We note that $m^{\prime}$ is real if $p$ and $q$ satisfy

$$
p^{2}+q^{2} \leq\left(\frac{|\vec{n}|}{n_{0}}\right)^{2}
$$

However, when $|\vec{n}|<n_{0}, m^{\prime}$ becomes imaginary if $p$ and $q$ satisfy

$$
\left(\frac{|\vec{n}|}{n_{0}}\right)^{2}<p^{2}+q^{2} \leq 1
$$

Here the imaginary $\mathrm{m}^{\prime}$ is denoted by

$$
m^{\prime}=i m_{i}^{\prime}=\sqrt{\left(\frac{n_{p}}{|n|}\right)^{2}\left(p^{2}+q^{2}\right)-1} \text {. }
$$

This imaginary $\mathrm{m}^{\prime}$ is related to the case of total internal reflection which will be discussed later. 
In order to derive Snell's law, as usually expressed, we consider the relationship between the three real unit vectors $, \hat{S}=(p, q, m), \hat{S}^{\prime}=\left(p^{\prime}, q^{\prime}, m^{\prime}\right)$ and $\hat{z}$. They are expressed in terms of spherical co-ordinates (see Eq. (3.12)), respectively, as

$$
\hat{S}=(p, q, m)=(\sin \theta \cos \phi, \sin \theta \sin \phi, \cos \theta)
$$

and

$$
\hat{s}^{\prime}=\left(p^{\prime}, q^{\prime}, m^{\prime}\right)=\left(\sin \theta^{\prime} \cos \phi^{\prime}, \sin \theta^{\prime} \sin \phi^{\prime}, \cos \theta^{\prime}\right)
$$

The vanishing triple scaler product, $\left[\hat{s} \cdot\left(\hat{s}^{\prime} \times \hat{z}\right)\right]=0$, shows that the unit vectors, $\hat{S}, \hat{S}^{\prime}$ and $\hat{z}$ are coplanar. We will call this plane the plane of incidence. We choose this plane such that the angles, $\phi$ and $\phi^{\prime}$, are equal to zero. Denoting by $\theta$ and $\theta^{\prime}$ the incident and the refracted angle respectively, and using one of the necessary conditions for the extinction of the incident wave, i.e., Eq. (3.26), we obtain Snell's law,

$$
|\vec{n}| \sin \theta^{\prime}=n_{0} \sin \theta
$$

where $|\vec{n}|$ is regarded as the refractive index of the absorbing medium.

The refractive index $|\vec{n}|$ can be expressed in terms of the real and imaginary part of the complex refractive index, $n_{r}$ and $n_{i}$, as well as the refractive index of the medium $M_{0}$. $n_{0}$, and the angle of incidence, $\theta$. In order to do this, we must first note the relations,

$$
n^{2}=n_{r}^{2}-n_{i}^{2}+2 i n_{r} n_{i}
$$

and

$$
\begin{aligned}
n^{2} & =n_{x}^{2}+n_{y}^{2}+n_{z}^{2} \quad\left(n_{z}=n_{z}^{r}+i n_{z}^{i}\right) \\
& =|\vec{n}|^{2}-\left(n_{z}^{i}\right)^{2}+2 i n_{z}^{i} n_{z}^{r} .
\end{aligned}
$$

Then, by comparing Eq. (3.29a) with Eq. (3.29b), we obtain 


$$
n_{r}^{2}-n_{i}^{2}=|\vec{n}|^{2}-\left(n_{i}^{i}\right)^{2}
$$

and

$$
n_{z}^{i}=\frac{n_{r} n_{i}}{n_{z}^{r}}=\frac{n_{r} n_{i}}{|\vec{n}| \cos \theta^{\prime}}
$$

Substituting Eq. (3.30b) into Eq. (3.30a), we find

$$
|\vec{n}|^{4} \cos ^{2} \theta^{\prime}-|\vec{n}|^{2} \cos ^{2} \theta^{\prime}\left(n_{r}^{2}-n_{i}^{2}\right)-n_{r}^{2} n_{i}^{2}=0
$$

in terms of the refracted angle $\theta^{\prime}$.

Using Snell's law, it can be further written in terms of the incident angle $\theta$,

$$
|\vec{n}|^{4}-|\vec{n}|^{2}\left(n_{r}^{2}-n_{i}^{2}+n_{0}^{2} \sin ^{2} \theta\right)+n_{0}^{2} \sin ^{2} \theta\left(n_{r}^{2}-n_{i}^{2}\right)-n_{r}^{2} n_{i}^{2}=0 .
$$

Solving this, we obtain

$$
|\vec{n}|=\left\{\frac{1}{2}\left(n_{r}^{2}-n_{i}^{2}+n_{0}^{2} \sin ^{2} \theta\right)+\frac{1}{2}\left[\left(n_{r}^{2}-n_{i}^{2}-n_{0}^{2} \sin ^{2} \theta\right)^{2}+4 n_{r}^{2} n_{i}^{2}\right)^{1 / 2}\right\}^{1 / 2} \text {. }
$$

We now consider the case where $\mathrm{m}$ ' becomes imaginary. In this case the propagation direction of the wave in Eq. (3.13) is along a new vector,

$$
\left(\vec{n}\left|p^{\prime},\right| \vec{n} \mid q^{\prime}, n_{z}^{i}\right) \text {, }
$$

where $n_{z}^{i}=n_{r} n_{i} /\left(|\vec{n}| m_{i}^{\prime}\right)$ (see Eq. $\left.(3.30 b)\right)$. We therefore define the new propagation direction vector for this case, as

$$
\begin{aligned}
\vec{n}^{\prime \prime} & =\left(n_{x}^{\prime \prime}, n_{y}^{\prime \prime}, n_{z}^{\prime \prime}\right) \\
& \left.=\mid \vec{n}^{\prime \prime}\right)\left(\sin \theta^{\prime \prime} \cos \phi^{\prime \prime}, \sin \theta^{\prime \prime} \sin \phi^{\prime \prime}, \cos \theta^{\prime \prime}\right) \\
& =\left|\vec{n}^{\prime \prime}\right|\left(p^{\prime \prime}, q^{\prime \prime}, m^{\prime \prime}\right),
\end{aligned}
$$

where the real unit vector is $\hat{S}^{\prime \prime}=\left(p^{\prime \prime}, q^{\prime \prime}, m^{\prime \prime}\right)$.

The relation between Eqs. (3.32) and (3.33) is

$$
p^{\prime \prime}=\frac{|\vec{n}|}{\left|\vec{n}^{\prime \prime}\right|} p^{\prime}, q^{\prime \prime}=\frac{|\vec{n}|}{\left|\vec{n}^{\prime \prime}\right|} q^{\prime} \text { and } m^{\prime \prime}=\frac{1}{\left|\vec{n}^{\prime \prime}\right|} \cdot \frac{n_{r} n_{i}}{|\vec{n}| m_{i}^{\prime}} \quad\left(m^{\prime \prime}=\sqrt{1-\left(p^{\prime 2}+q^{\prime \prime 2}\right)}\right) \text {. }
$$

The imaginary $\mathrm{m}^{\prime}$, corresponds to the case of total internal reflection in the absorbing medium. However, because of the 
imaginary part of the complex refractive index $n(\omega)$, we do not have the usual surface waves in total internal reflection. Instead, we have the wave propagating along the new direction of $\vec{n}$ " and Snell's law in terms of the refractive index $\left|\vec{n}^{\prime \prime}\right|$. Noting that the plane of incidence is specified by $\phi^{\prime \prime}=0$ and that $\theta^{\prime \prime}$ is the refracted angle, we obtain Shell's law,

$$
n_{0} \sin \theta=\left|\vec{n}^{\prime \prime}\right| \sin \theta^{\prime \prime} \text {. }
$$

In order to express the refractive index, $\left|\vec{n}^{\prime \prime}\right|$, in terms of $n_{r}, n_{i}, n_{0},|\vec{n}|$ and $\theta$, we first note the relation,

$$
m^{\prime \prime}=\sqrt{1-\left(p^{\prime \prime 2}+q^{\prime \prime 2}\right)}=\sqrt{1-\sin ^{2} \theta^{\prime \prime}} \text {. }
$$

Using Snell's law, Eq. (3.35), we then find

$$
m^{\prime \prime}=\frac{1}{\left|\vec{n}^{\prime \prime}\right|}\left(\left|\vec{n}^{\prime \prime}\right|^{2}-n_{0}^{2} \sin ^{2} \theta\right)^{1 / 2}
$$

On the other hand, using Eq. (3.34), we obtain

$$
\begin{aligned}
m^{\prime \prime} & =\frac{1}{\left|\vec{n}^{\prime \prime}\right|} \frac{n_{r} n_{i}}{m^{\prime}} \\
& =\frac{1}{\left|\vec{n}^{\prime \prime}\right|} n_{r} n_{i}\left(n_{0}^{2} \sin ^{2} \theta-|\vec{n}|^{2}\right)^{-1 / 2} .
\end{aligned}
$$

Equating Eqs. $(3.36)$ and $(3.37)$, we have

$$
\left.\vec{n}^{\prime \prime}\right|^{2}-n_{0}^{2} \sin ^{2} \theta=n_{r}^{2} n_{i}^{2} /\left(n_{0}^{2} \sin ^{2} \theta-|\vec{n}|^{2}\right) \text {, }
$$

so that $\left|\vec{n}^{\prime \prime}\right|$ can be written as

$$
\left|\vec{n}^{\prime \prime}\right|=\left\{n_{r}^{2} n_{i}^{2} /\left(n_{0}^{2} \sin ^{2} \theta-|\vec{n}|^{2}\right)+n_{0}^{2} \sin ^{2} \theta\right\}^{1 / 2}
$$

To derive Fresnel's formula, we define the polarization states for the plane waves; one is perpendicular and the other is parallel to the plane of incidence. We denote them by unit vectors $\hat{\lambda}_{\perp}$ and $\hat{\lambda}_{/ /}$respectively. Since the electromagnetic waves are inhomogeneous in the absorbing medium $\mathbb{M}_{1}$, the 
relationships between the $\hat{\lambda}_{1 \prime}$ states and the propagation directions (for instance, $\hat{S}^{\prime}=\left(p^{\prime}, q^{\prime}, m^{\prime}\right)$ ) are complicated. But the behavior of the perpendicular components of fields at refraction is simple. Namely, if the polarization state for the incident electric vector amplitudes, $\vec{e}^{(i)}(p, q, \omega)$, is in the $\hat{\lambda}_{\perp}$ state, the reflected and refracted vector amplitudes, $\vec{e}^{(r)}(\mathrm{p}, \mathrm{q}, \omega)$ and $\vec{e}\left(\mathrm{p}^{\prime}, \mathrm{q}^{\prime}, \omega\right)$, are also in the $\hat{\lambda}_{\perp}$ state. Thus,

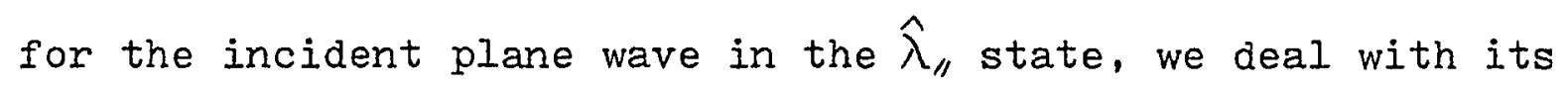
magnetic field which is in the $\hat{\lambda}_{\perp}$ polarization state. Assuming that $\vec{e}^{(i)}(p, q, w)$ is in the $\hat{\lambda}_{\perp}$, taking first the dot product of $\hat{\lambda}_{1}$ with Eqs. (3.21a) and (3.21b) from their left sides and then using the following relations,

$$
\hat{\lambda}_{\perp} \cdot \hat{S}=\hat{\lambda}_{\perp} \cdot \hat{S}^{(r)}=0
$$

and

$$
\hat{S} \cdot \vec{e}\left(p^{\prime}, q^{\prime}, \omega\right)=\hat{S}^{(r)} \cdot \vec{e}^{(r)}(p, q, \omega)=0,
$$

we obtain

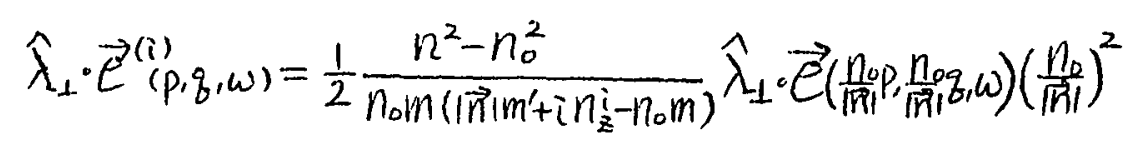

and

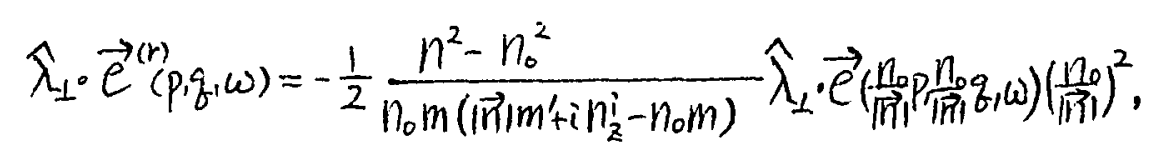

where $p^{2}+q^{2} \leq 1$ and $n_{z}^{i}=\frac{n_{r} n_{i}}{\tilde{n}_{1} \mid m^{\prime}}$. We note that $m^{\prime}$ is either real or pure imaginary depending upon the incident direction cosines (see Eqs. (3.27) and (3.28)).

Thus, for the case where the polarization state of $\vec{e}^{(i)}(p, q, \omega)$ is perpendicular to the plane of incidence, we obtain the transmission and the reflection amplitude, $b_{T_{\perp}}$ 
and $\delta_{R_{\perp}}$ respectively, as

$$
b_{T_{\perp}}=\frac{\hat{\lambda}_{\perp} \cdot \vec{e}(p, q, \omega)}{\hat{\lambda}_{\perp} \cdot \vec{e}^{(i)}(p, q, \omega)}=\frac{2 n_{0} m\left(|\vec{n}| m^{\prime}+i \frac{n_{r} n_{i}}{\left|\hat{n}^{\prime}\right| m^{\prime}}-n_{0} m\right)}{n^{2}-n_{0}^{2}}
$$

and

$$
b_{R_{+}}=\frac{\hat{\lambda}_{\perp} \cdot \vec{e}^{(r)}(p, q, \omega)}{\hat{\lambda}_{\perp} \cdot \vec{e}^{(i)}(p, q, \omega)}=\frac{|\vec{n}| m^{\prime}+i \frac{n_{r} n_{i}}{|\vec{n}| m_{i}^{\prime}}-n_{0} m}{|\vec{n}| m^{\prime}+i \frac{n_{r} n_{i}}{|\vec{n}| m^{\prime}}+n_{0} m},
$$

where the subscript, $\perp$, refers to the perpendicular polarization state of $\vec{e}^{(i)}$. These can be expressed in terms of the incident angle $\theta$, if we note the following relation,

$$
\left(\overrightarrow{m i} m^{\prime}+i \frac{n_{r} n_{i}}{\vec{m} \mid m^{\prime}}\right)^{2}=n^{2}-n_{0}^{2} \sin ^{2} \theta,
$$

where $n(\omega)$ is the complex refractive index for the medium $M_{1}$ and $n_{0}$ is the refractive index for $M_{0}$. We then have

$$
\sigma_{T_{\perp}}=\frac{2 n_{0} \cos \theta}{n_{0} \cos \theta+\sqrt{n^{2}-n_{0}^{2} \sin ^{2} \theta}}
$$

and

$$
\sigma_{R_{1}}=\frac{n_{0} \cos \theta-\sqrt{n^{2}-n_{0}^{2} \sin ^{2} \theta}}{n_{0} \cos \theta+\sqrt{n^{2}-n_{0}^{2} \sin ^{2} \theta}} \text {. }
$$

We now assume that the polarization of $\vec{e}^{(i)}(p, q, \omega)$ is parallel to the plane of incidence. The polarization state of $\vec{h}^{(i)}(p, q, \omega)$ is now perpendicular. Taking the dot product of $\hat{\lambda}_{1}$ with Eqs. (3.22b) and (3.22c) from their lefthand sides and then using the relation of Eq. (3.25), we obtain

$$
\hat{\lambda}_{\perp} \cdot \vec{h}_{h}^{(i)}\left(p_{1}, \omega\right)=\frac{1}{2} \frac{n^{2}-n_{0}^{2}}{n^{2}} \frac{|\vec{n}|\left(\hat{s} \cdot \hat{s}^{\prime}\right)+i \frac{n_{r} n_{i}}{|\vec{n}| m^{\prime}},(\hat{s}-\hat{z})}{m\left(|\vec{n}| m^{\prime}+i \frac{n_{r} n_{i}}{|\vec{n}| m^{\prime}}-n_{0}(m)\right.} \hat{\lambda}_{\perp} \cdot \vec{h}\left(\frac{n_{0}}{|\vec{n}|} \theta, \frac{n_{0}}{|\vec{n}|}, q, \omega\right)\left(\frac{n_{0}}{|\vec{n}|)^{2}}\right.
$$


and

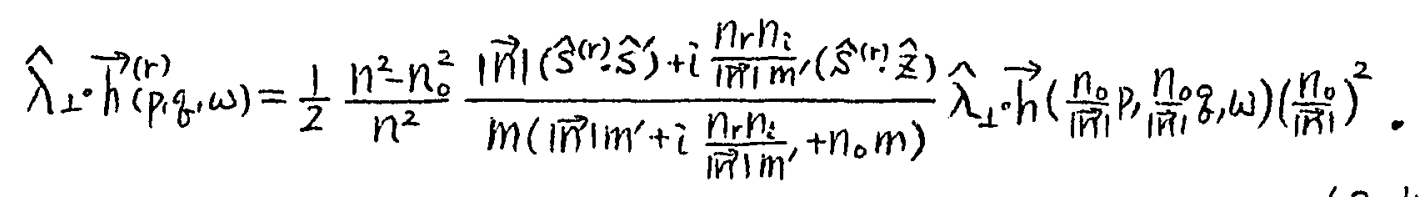

Using Eq. (3.40) and noting that $\hat{S} \cdot \hat{S}^{\prime}=\cos \left(\theta^{\prime}-\theta\right)$ and $\hat{S} \cdot \hat{Z}=\cos \theta$, the transmission and reflection amplitudes, $\pi_{T_{\text {r }}}$ and $\Pi_{R_{1 \prime}}$, are

$$
\begin{aligned}
\pi_{T^{\prime \prime}}=\frac{\hat{\lambda}_{+} \cdot \vec{h}\left(p^{\prime}, q^{\prime}, \omega\right)}{\hat{\lambda}_{+} \cdot \vec{h}^{(i)}(p, q, \omega)} & =\frac{2 n^{2} m}{n^{2}-n_{0}^{2}} \frac{\vec{n} \mid m^{\prime}+i \frac{n_{r} n_{i}}{|\vec{n}| m^{\prime}}-n_{0} m}{|\vec{n}|\left(\hat{s} \cdot \hat{\vec{s}}^{\prime}\right)+i \frac{n_{r} n_{i}}{\vec{n} \mid m^{\prime}}(\hat{\vec{s}} \cdot \hat{z})} \\
& =\frac{2 n^{2} \cos \theta}{n^{2} \cos \theta+n_{0} \sqrt{n^{2}-n_{0}^{2} \sin ^{2} \theta}}
\end{aligned}
$$

and

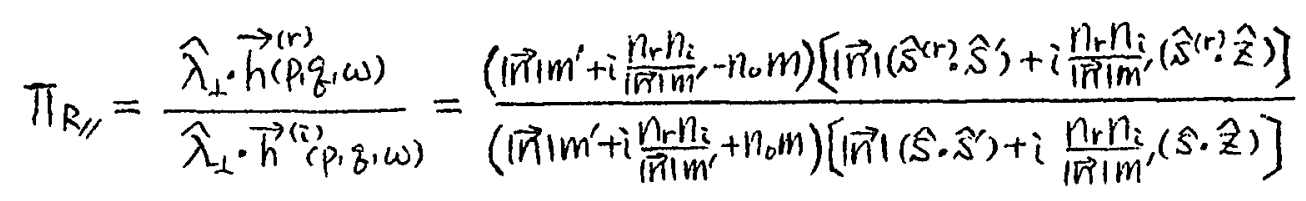

$$
\begin{aligned}
& =\frac{n^{2} \cos \theta-n_{0} \sqrt{n^{2}-n_{0}^{2} \sin ^{2} \theta}}{n^{2} \cos \theta+n_{0} \sqrt{n^{2}-n_{0}^{2} \sin ^{2} \theta}} \text {. }
\end{aligned}
$$

Here the subscript, // , refers to the parallel polarization state of the incident vector amplitude of $\vec{e}^{(i)}(p, q, \omega)$. The incident electromagnetic wave, $\vec{E}^{(i)}(\vec{r}, \omega)$ with $\vec{H}^{(i)}(\vec{r}, \omega)$, which is expressed in terms of a bundle of plane waves at various incident angles, can also be related uniquely to the corresponding reflected and refracted waves. If the polarization state of $\vec{E}^{(i)}(\vec{r}, \omega)$ is perpendicular to the 
plane of incidence, using the reflection amplitude of Eq. $(3.41 \mathrm{~b})$, the reflected field of $\mathrm{Eq} .(3.5 \mathrm{~b})$ can be written as

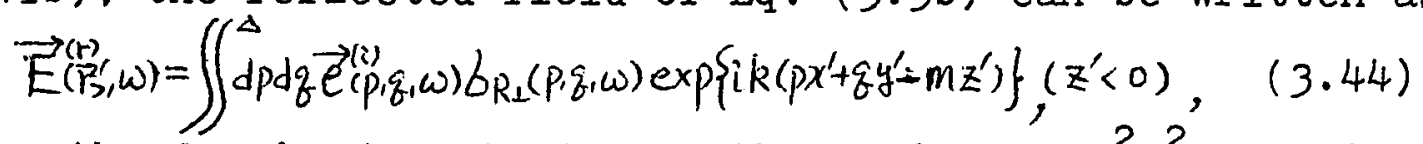
where the domain, $\Delta$, stands for the region of $p^{2}+q^{2} \leq 1$. Similarly, using the refraction amplitude of $\mathrm{Eq} .(3.41 \mathrm{a})$, the refracted field of Eq. (3.13) can be written as

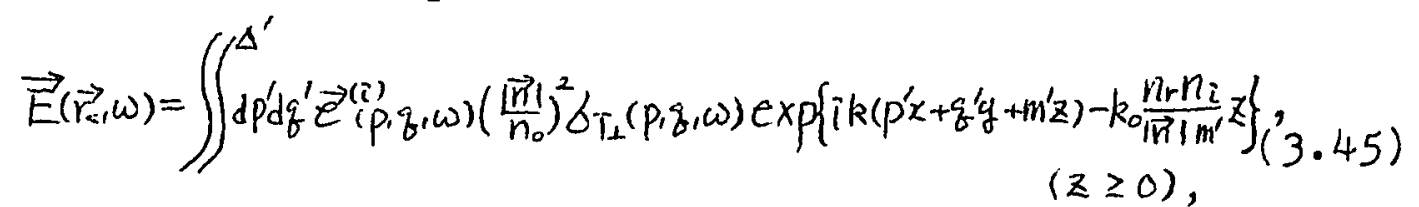

where

$$
p=\frac{|\vec{n}|}{n_{0}} p^{\prime}, q=\frac{|\vec{n}|}{n_{0}} q^{\prime}
$$

and the domain, $\Delta^{\prime}$, stands for the region of $p^{\prime 2}+q^{\prime 2}=\left(\frac{n_{0}}{\sqrt{n^{2}}}\right)^{2}\left(p^{2}+q^{2}\right) \leq\left(\frac{n_{0}}{\mid \vec{p})^{2}}\right)^{2}$. We note that the domain, $\Delta^{\prime}$, includes the case of an imaginary $m^{\prime}\left(m^{\prime}=i m_{\bar{c}}^{\prime}\right)$.

If the polarization state of $\vec{E}^{(2)}\left(\vec{r}^{\prime}, \omega\right)$ is parallel to the plane of incidence, the state of $\overrightarrow{\mathrm{H}}^{(i)}\left(\vec{r}^{\prime}, \omega\right)$ is perpendicular. We now look for the reflected and refracted waves in terms of the magnetic fields. They are, respectively

$$
\vec{H}^{(r)}\left(\vec{r}^{\prime}, \omega\right)=\iint^{\Delta} d p d q \vec{h}^{(i)}(p, q, \omega) \Pi_{R^{\prime \prime}}(p, q, \omega) \exp \left\{i k\left(p x^{\prime}+q y^{\prime}-m z^{\prime}\right)\right\},\left(z^{\prime}<0\right),(3.46 a)
$$

and

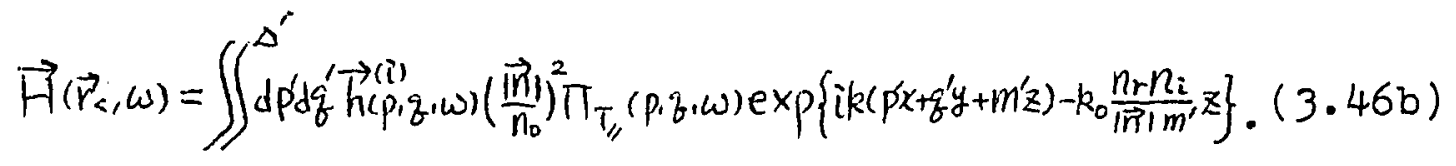

The reflected and refracted electromagnetic waves become unique when the functional form of the incident vector amplitudes, $\vec{e}^{(i)}(p, q, \omega)$ and $\vec{h}^{(i)}(p, q, \omega)$, are given. For instance, if the incident electromagnetic wave is a plane wave of a fixed mode, $\left(p_{0}, q_{0}, m_{0}\right), i . e .$, with an incident angle $\theta_{0}$, we can write the vector amplitudes, $\vec{e}^{(i)}(p, q, \omega)$ and $\vec{h}^{(i)}(p, q, \omega)$, using Dirac delta functions. They are 


$$
\vec{e}^{(i)}(p, q, \omega)=\vec{\varepsilon}^{(i)}(\omega) \delta\left(p-p_{0}\right) \delta\left(q-q_{0}\right)
$$

and

$$
\vec{h}^{(i)}(p, q, \omega)=\overrightarrow{X P}^{(i)}(\omega) \delta\left(p-p_{0}\right) \delta\left(q-q_{0}\right) .
$$

For this case, we summarize Fresnel's formulas and the reflection coefficients in the following way. If $\vec{\varepsilon}^{(i)}(\omega)$ is in the state, $\hat{\lambda}_{1}$, then we find

and

$$
\begin{aligned}
& \sigma_{T_{1}}\left(\theta_{0}, \omega\right)=\frac{2 n_{0} \cos \theta_{0}}{n_{0} \cos \theta_{0}+\sqrt{n^{2}-n_{0}^{2} \sin ^{2} \theta_{0}}}, \\
& \sigma_{R_{1}}\left(\theta_{0}, \omega\right)=\frac{n_{0} \cos \theta_{0}-\sqrt{n^{2}-n_{0}^{2} \sin ^{2} \theta_{0}}}{n_{0} \cos \theta_{0}+\sqrt{n^{2}-n_{0}^{2} \sin ^{2} \theta_{0}}}
\end{aligned}
$$

$$
R_{\perp}=\left|\sigma_{R_{1}}\right|^{2}=\left|\frac{n_{0} \cos \theta_{0}-\sqrt{n^{2}-n_{0}^{2} \sin ^{2} \theta_{0}}}{n_{0} \cos \theta_{0}+\sqrt{n^{2}-n_{0}^{2} \sin ^{2} \theta_{0}}}\right|^{2} .
$$

On the other hand, if $\vec{\varepsilon}^{(2)}(\omega)$ is in the state, $\hat{\lambda}_{11}$, then

and

$$
\begin{aligned}
& \pi_{\perp 11}\left(\theta_{0}, \omega\right)=\frac{2 n^{2} \cos \theta_{0}}{n^{2} \cos \theta_{0}+n_{0} \sqrt{n^{2}-n_{0}^{2} \sin ^{2} \theta_{0}}}, \\
& \pi_{R_{11}}\left(\theta_{0}, \omega\right)=\frac{n^{2} \cos \theta_{0}-n_{0} \sqrt{n^{2}-n_{0}^{2} \sin ^{2} \theta_{0}}}{n^{2} \cos \theta_{0}+n_{0} \sqrt{n^{2}-n_{0}^{2} \sin ^{2} \theta_{0}}}
\end{aligned}
$$

$$
R_{/ I}=\left|\Pi_{R / /}\left(\theta_{0}, \omega\right)\right|^{2}=\left|\frac{n^{2} \cos \theta_{0}-n_{0} \sqrt{n^{2}-n_{0}^{2} \sin ^{2} \theta_{0}}}{n^{2} \cos \theta_{0}+n_{0} \sqrt{n^{2}-n_{0}^{2} \sin ^{2} \theta_{0}}}\right|^{2} .
$$

In the expressions above, the complex refractive index, $n(\omega)$, is for the transition region just inside the surface of the absorbing material. The complex refractive index may be different from that of the bulk material depending upon the degree of the surface effect. The detailed analysis of this surface effect on the refractive index will be discussed 
in the following two chapters. We note that we still have the same Fresnel formulas, even when the angle of incidence satisfies the condition for total internal reflection,

$$
1 \geq \sin ^{2} \theta_{0}>\left(\frac{|\vec{n}|^{2}}{n_{0}}\right)^{2}, \quad\left(|\vec{n}|<n_{0}\right) \text {. }
$$

\section{A DERIVATION OF FRESNEL'S FORNIULA FOR THE CASE OF A SHARP BOUNDARY}

In this section, we consider the limiting case of a vanishingly small thickness of the transition region, $V_{ \pm}$. We redenote by $S$ the plane boundary, $S-,(x-y$ plane $)$ in that limit and place it at $z=0$. Then our formal solutions at $\vec{r}^{\prime}=\left(x^{\prime}, y^{\prime}, z^{\prime}\right)$, Eqs. $(3.1 a)$ and $(3.1 b)$, can be written as

$$
\vec{E}^{\prime}\left(\vec{r},(\omega)+\frac{1}{4 \pi k^{2}} \nabla^{\prime} \times \nabla^{\prime} \times \int_{S} d S\left\{\vec{E}(\vec{r}, \omega) \frac{\partial}{\partial n} g\left(\vec{r} \mid \vec{r}^{\prime} ; k\right)-g\left(\vec{r} \mid \vec{r}^{\prime} ; k\right) \frac{\partial}{\partial n} \vec{E}(\vec{r}, \omega)\right\}=\left\{\begin{array}{l}
\vec{E}\left(\vec{r}_{>}^{\prime}, \omega\right), z^{\prime}<0, \\
0, z^{\prime}>0,(3.50 a)
\end{array}\right.\right.
$$

and

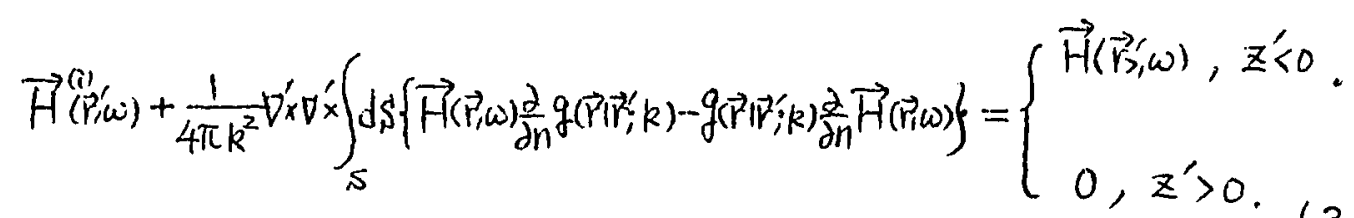

Using plane wave mode expansions and denoting $(p, q, m)$ and $(p, q,-m)$ by $\hat{s}$ and $\hat{s}^{(r)}$ respectively, we can express Eq. (3.50a) in the fully non-local form in space;

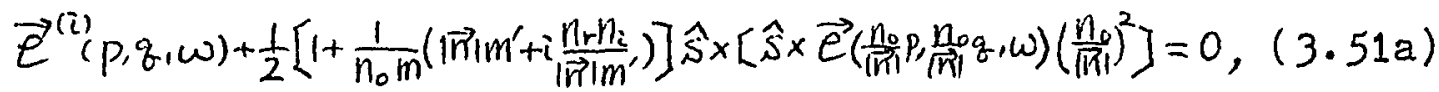

$$
\begin{aligned}
& \vec{e}^{(\text {out })}(p, q, \omega)=\vec{e}^{(i)}(p, q, \omega)+\vec{e}^{(r)}(p, q, \omega),
\end{aligned}
$$

where 


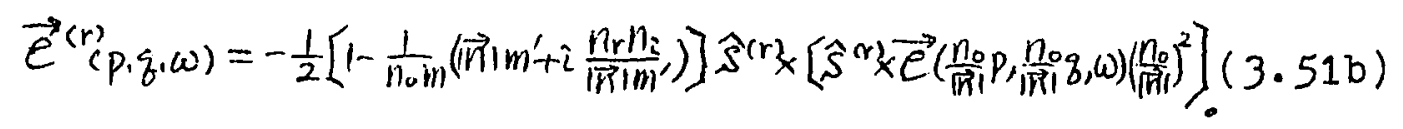

Here we note that Eqs. (3.50a) and $(3.50 \mathrm{~b})$ can be reduced to Eqs. (3.21a) and (3.21b) respectively if we use the following identity obtained from Eq. (3.40),

$$
\left(n^{2}-n_{0}^{2}\right) /\left(|\vec{n}| m^{\prime}+i \frac{n_{r} n_{i}}{|\vec{n}| m^{\prime}} \mp n_{0} m\right)=|\vec{n}| m^{\prime}+i \frac{n_{r} n_{i}}{|\vec{n}| m^{\prime}} \pm n_{0} m .
$$

Similarly, noting that $\hat{n}=-\hat{z}, E q .(3.50 b)$ can be written as

$$
\begin{aligned}
& \vec{h}(p, q, \omega)+\frac{1}{2}\left[1+\frac{1}{n_{0} m}\left(|\vec{n}| m^{\prime}+i \frac{n_{r} n_{i}}{\left|\vec{n}^{\prime}\right| m^{\prime}}\right)\right] \hat{s} \times\left[\hat{s} \times \vec{h}\left(\frac{n_{0}}{\mid \overrightarrow{\mid \overrightarrow{ }} P} p \frac{n_{0}}{|\vec{n}|} q, \omega\right)\left(\frac{n_{0}}{\mid \overrightarrow{\vec{n} \mid})^{2}}\right)^{2}\right]
\end{aligned}
$$

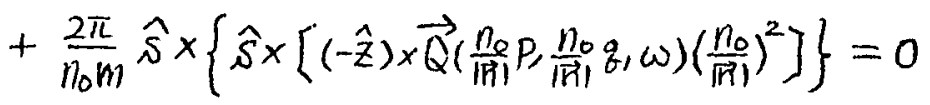

and

$$
\vec{h}^{(\text {ouct })}(p, q, \omega)=\vec{h}^{(i)}(p, q, \omega)+\vec{h}^{(r)}(p, q, \omega),
$$

where

$$
\begin{aligned}
& \vec{h}^{(r)}(p, q, \omega)=-\frac{1}{2}\left[1-\frac{1}{n_{0} m}\left(|\vec{n}| m^{\prime}+i \frac{n_{r} n_{i}}{\left|\vec{n}^{\prime}\right| m^{\prime}}\right)\right] \hat{s}^{(r)} \times\left[\hat{s}^{(r)} \times \vec{h}\left(\frac{n_{e} p}{|\vec{m}|}, \frac{n_{0}}{|\vec{n}| q, \omega)}\left(\frac{n_{0}}{|\vec{n}|}\right)^{2}\right]\right. \\
& +\frac{2 \pi}{n_{0} m} \hat{s}^{(r)} \times\left\{\hat{S}^{(r)} \times(-\hat{z}) \times \vec{Q}\left(\frac{n_{0}}{|\vec{n}|} p, \frac{n_{0}}{\mid \vec{n}}\right)^{q}, w\right)\left\langle\frac{n_{0}}{\mid \overrightarrow{|r|})^{2}}\right\} \text {. }
\end{aligned}
$$

Here we can relate the terms, $-\hat{z} \times \vec{Q}$, in Eqs. (3.53a) and (3.53b) to the magnetic vector amplitude $\vec{h}$ through a relation similar to $\mathrm{Eq} \cdot(3.25)$.

In order to obtain Fresnel's formulas, we will follow the procedure of the previous section. Then, because of the relation of Eq. (3.52), we find exactiy the same expressions for the reflection and the refraction amplitudes as before. When the incident wave is assumed to be a plane wave of a 
fixed mode, $\left(p_{0}, q_{0}, m_{0}\right)$, i.e., for the incident angle $\theta_{0}$, we obtain the same expressions for Fresnell's formulas and the reflection coefficients, (see Eqs. (3.48) and (3.49)) with the complex refractive index $n(\omega)$ for the bulk material, as before. Then, these results agree exactly with the results which are obtained by the usual treatment, for instance, in the text of I. D. Landau and E. M. Lifshitz (1960). Finally replacing the medium $M_{0}$ with vacuum and $M_{1}$ with non-absorbing dielectric, i.e., $n_{0}=1$ and $n_{i}$ (the imaginary part of $\left.n(\omega)\right)=0$, our model reduces to that studied by $E$. Lalor and $E$. Wolf (1972). 
CHAPTER IV

\section{SEIECTIVE REFLECTION OF LIGHT \\ FRON A SOLID-GAS INTERFACE}

\section{INTRODUCTION}

We now consider a system consisting of Na vapor contained in a quartz bulb a part of which consists of a slightIy prismatic glass plate (see the text of R. W. Wood, 1934). We wish to study the selective reflection of light from the vapor at the prismatic plate. This plate is made prismatic to prevent interference effects of the light reflected from the inner and the outer surface of the plate. However, in order to simplify the theoretical treatment of the system, we consider it to consist of a $\mathrm{Na}$ vapor which fills the semiinfinite half space $(z \geq 0)$ and a glass material which occupies the other semi-infinite half space $(z<0)$.

J. P. Woerdman and M. F. H. Schuurmans (1974) used a tunable laser beam to experimentally study the selective reflection by using a system very similar to the above idealized system. An incident beam with a narrow spectral width was introduced from the glass side. They obtained the line shapes of the reflected light, when the incident beam was swept across the strong resonance lines, $D_{1}\left({ }^{2} S_{1 / 2}-{ }^{2} P_{1 / 2}, 5896 A^{\circ}\right)$ and $D_{2}\left({ }^{2} S_{1 / 2}-{ }^{2} P_{3 / 2}, 5890 A^{\circ}\right)$. Various incident angles less than the critical angle were used. In their experiments, the gas 
temperature was approximately $600 \mathrm{~K}$ so that the density of atoms in the gas was about $10^{15} \mathrm{~cm}^{-3}$.

They observed the following phenomena. A hyperfine doublet splitting (1772 MHz) in each of the $\mathrm{Na} D$ lines was observed when the light was reflected normally. The resolution decreased as the incident angle increased until the splitting was completely masked by the Doppler broadening. We note that the hyperfine splitting of $1772 \mathrm{MHz}$ is approximately equal to the Doppler half-width (2000 $\mathrm{MHz}$ ) expected under their experimental conditions (see Fig. 4.1).

We will develop a theory to explain this phenomena based on the following model. Because of the strong resonance absorption of $\mathrm{Na}$ atoms, the penetration depth of the incident light is expected to be less than the kinetic mean free path of the Na atoms. Thus only the gaseous atoms near the wall affect the reflection. The motion of atoms near the wall may be isotropic. However, those atoms which are moving in a direction nearly perpendicular to the wall will spend only a short time in the penetration region. On the other hand, those atoms moving nearly parallel to the wall will remain in the region for at least the mean free time before being deflected out at their next collision. Therefore the atoms contributing significantly to the reflected light are those which are moving nearly parallel to the wall. As a result, when the angle of incidence is increased, the Doppler broadening of the reflected light becomes greater. In order to apply the extinction theorem to our model, 


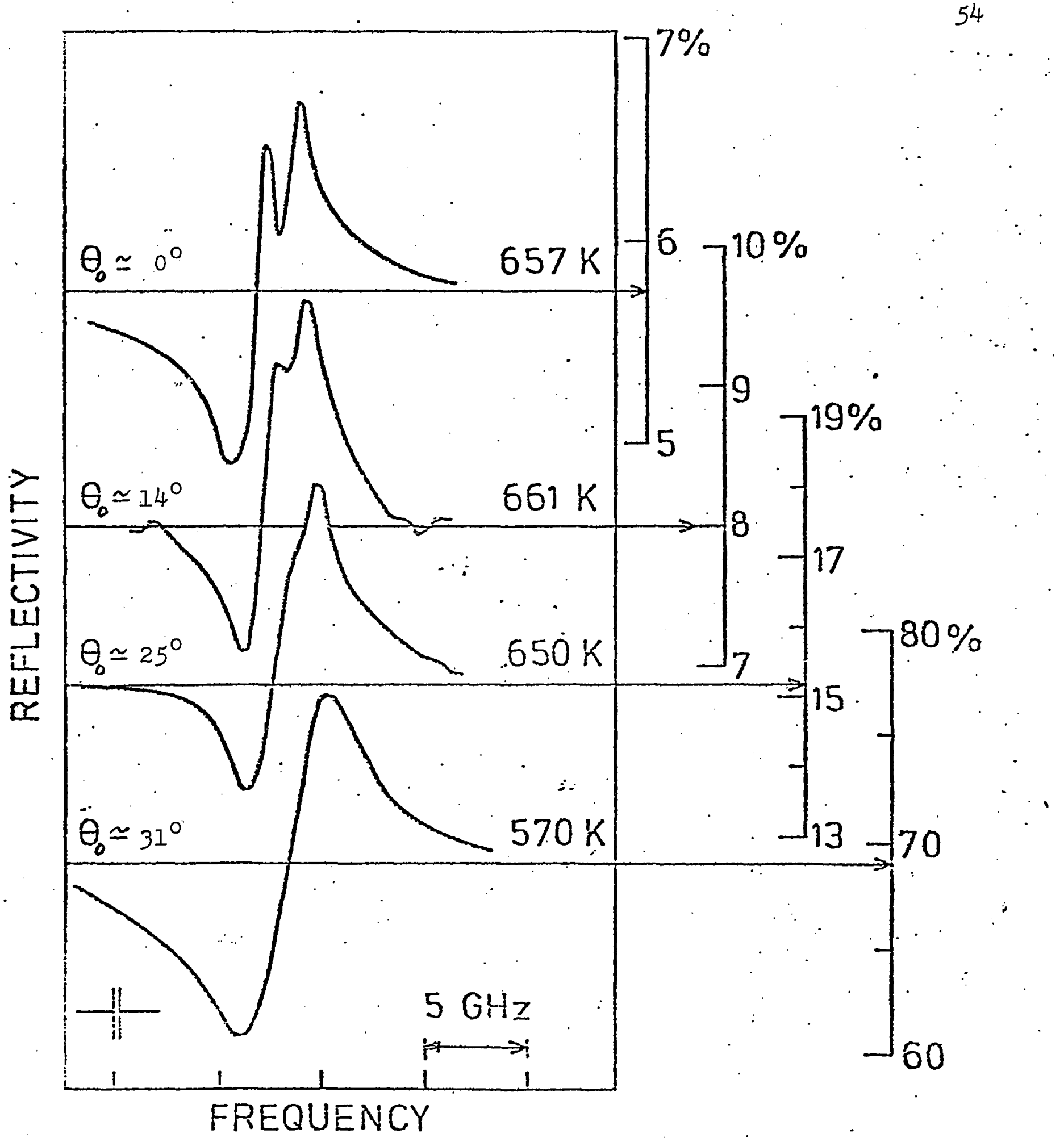

Fig. 4.1 Measured reflectivity of a.gehlenite glasssodium vapor interface near the $\mathrm{D}_{2}$ line for various incident angles $\theta_{0}$ 's less than the critical angle (J. P. Woerdman and M. F. H. Schuurmans, 1974). 
we separate our system into regions by placing the hypothetical surface $S+$ at the wall and $S$ - at a distance deep enough that all the field quantities vanish. This is because of the strong resonance absorption within the transition region $\mathrm{V}_{ \pm}$. In this region $\mathrm{V}_{ \pm}$, there are atoms which have been direc -tly reflected from the wall. Since the polarizability depends strongly on frequencies near a resonance, the induced dipole moment depends on the past history of the atoms, including those reflected from the wall. To obtain the macroscopic fields, we must average the fields over the past behavior of the atoms. This averaging will be discussed in section 2. The rest of the chapter is then organized as fol -lows. In section 3, expressions of the line shape of the reflected light used in the numerical calculations are given. The computer program used for these calculations and the results are given in Appendix A.

\section{THE MACROSCOPIC POLARIZATION FIELD WITHIN THE PENETRATION IAYER}

Let us consider the system of the glass and $\mathrm{Na}$ vapor which was introduced in section 1 . If the vapor is at room temperature without strong incident light, the majority of the $\mathrm{Na}$ atoms are in the ground state. In the presence of incident light some of the atoms will be raised to excited states by absorbing incident photons. These excited states have finite lifetimes which produce: the "natural line width" of the spectral lines emitted by the excited atoms. Also, 
since all the vapor atoms are in thermal motion, and an excited atom may pass near an unexcited atom, then there is a finite probability that the excitation energy will be transferred from the first to the second atom if their states can be radiatively connected. This results in a reduction of the duration of the coherent oscillation of light emitted by the excited atom and in a corresponding broadening of the emitted light. Line broadening of this type, as if due to "optical collisions", is known as "resonance broadening" and the line width is proportional to the density of $\mathrm{Na}$ atoms at low vapor pressure. In the response of the gas system to incident light, all of the above mechanism contribute to the frequency dependence of the induced polarization.

We can treat the polarization classically by replacing the excited $\mathrm{Na}$ atom with a damped harmonic oscillator of natural angular frequency $\omega_{c e}$. We will denote by $\gamma_{c}$ the damping constant of the oscillator which is proportional to the natural line width, and by $m_{e}$ and $e$ the mass and charge of the electron respectively. We find the equation of motion of the $x$ component of the displacement vector of the oscillating electron with oscillator strength $f_{0,}$ under the influence of an effective electric field, $e_{o x} e^{-i \omega t}$, to be

$$
\ddot{x}(t)+\gamma_{0}^{\prime} \dot{x}(t)+\omega_{02}^{2} x(t)=\frac{e}{m_{e}} f_{02} e_{0 x} e^{-i \omega t}
$$

with a solution, 


$$
x(t)=C_{1} e^{-i\left(\omega_{0 . e}-i \frac{\partial_{c}}{2}\right) t}+C_{2} e^{i\left(\omega_{0 R}+i \frac{\gamma_{c}}{2}\right) t}+\frac{e}{m_{e}} f_{0 . r} \frac{e_{0 x} e^{-i \omega t}}{\omega_{0,}^{2}-\omega^{2}-i \omega \gamma_{0}}{ }^{\prime}(4-1 b)
$$

where $C_{1}$ and $C_{2}$ are constants. The polarization is proportional to $x(t)$. In order to include effects of optical collisions, we must consider what happens immediately after the collisions (see a review article by $S$. Y. Chen and Mi. Takeo, 1957). The optical effect of collisions with a wall can be treated in the same way.

We assume that the collision duration is very short and the collision is "strong", when the energy transfer is likely to occur through resonance. After a strong collision the atom has no memory concerning its orientation or other distribution properties before the collision. Suppose that a collision takes place at time $t_{x}$. It is assumed, because of the random nature of collisions, that the averages of $x\left(t_{x}\right)$ and $\dot{x}\left(t_{x}\right)$ over the collisions are zero. This is known as the "Lorentz assumption". The solution of Eq. (4.1a), i.e., Eq. (4.1b), under this assumption becomes

$$
\begin{aligned}
x(t) & =\frac{e}{m_{e}} f_{0 e} \cdot \frac{e_{0 x}}{2 \omega_{c \ell}} \frac{e^{-i \omega t}}{\omega_{0 R}-\omega-i \cdot \frac{\gamma_{0}}{2}}\left\{1-e^{-i\left(\omega_{i 2}-\omega-i \frac{\gamma_{c}}{2}\right)\left(t-t_{x}\right)}\right\} \\
& +\frac{e}{m_{e}} f_{0 . \Omega} \cdot \frac{e_{0 x}}{2 \omega_{0 \Omega}} \frac{e^{-i \omega t}}{\omega_{0 \Omega}+\omega+i \frac{\gamma_{c}}{2}}\left\{1-e^{i\left(\omega+\omega_{0 e}+i \frac{\gamma_{0}}{2}\right)\left(t-t_{x}\right)}\right\} .
\end{aligned}
$$

For an effective electric field, $e_{o x}(t)$, this solution can be written as 
$x(t)=\frac{e}{m} f_{0 R} \int_{-\infty}^{+\infty} \frac{d \omega}{2 \pi} e^{-i \omega t} \frac{e_{0 x}(\omega)}{2 \omega_{0}}\left\{\frac{1-e^{-i\left(\omega_{0 R}-\omega-i \frac{\gamma_{c}}{2}\right)\left(t-t_{x}\right)}}{\omega_{i R}-\omega-i \gamma_{0 / 2}}-\frac{1-e^{i\left(\omega+\omega_{0 R}+i \frac{\gamma_{c}}{2}\right)\left(t-t_{x}\right)}}{\omega_{0 R}+\omega+i \gamma_{0 / 2}}\right\},(4.3)$

where $e_{o x}(t)$ is expressed in a Fourier integral form.

$$
e_{o x}(t)=\int_{-\infty}^{+\infty} \frac{d \omega}{2 \pi} e_{o x}(\omega) e^{-i \omega t}
$$

Denoting the electric dipole moment induced in the atom as $p_{x}(t)=e x(t)$ and the electric polarizability as $\alpha_{h}(t)$, then using the convolution theorem, the solution can be further rewritten as a linear response form of

where $\quad \alpha_{l}(t)=\frac{1}{2 \pi} \int_{-\infty}^{\infty} d \omega e^{-i \omega t} \alpha_{l}(\omega)$

$$
p_{x}(t)=\int_{t_{x} \text { is }}^{t} d \tau \alpha_{e}(t-\imath) e_{0 x}(\tau)
$$

with

$$
\alpha_{e}(\omega)=\frac{e^{2}}{m_{e}} \frac{f_{0 . e}}{\omega_{c 2}^{2}-\omega^{2}-i \omega \gamma_{0}} \text {. }
$$

Therefore, if the effective field $\vec{e}\left(\vec{r}_{a}, t\right)$ is nearly identical to the mean electric field $\vec{E}\left(\vec{r}_{a}, t\right)$ at $\vec{r}_{a}$ as in our model case, we obtain

$$
\vec{p}\left(\vec{r}_{a}, t\right)=\int_{t_{x}}^{t} d \hat{\imath} \alpha(t-\imath) \vec{E}\left(\vec{r}_{a}, \tau\right)
$$

where we recall that $t_{x}$ is the time of the last collision. We will write the subscript " $x$ " as " $c$ " if the last collision is with another atom and as "w" if the last collision is with the wall.

This polarized atom moves with a thermal velocity $\vec{v}_{a}=\left(v_{x}^{a}, v_{y}^{a}, v_{z}^{a}\right)$, so that the position of the atom at time $t$ can be written as

$$
\vec{r}_{a}(t)=\vec{r}_{a}\left(t_{x}\right)+\vec{u}_{a} \times\left(t-t_{x}\right),\left(\begin{array}{l}
\vec{r}_{a}\left(t_{x}\right)=\left(x_{c}, y_{c}, z_{c}\right) \\
\vec{r}_{a}(t)=\left(x_{a}, y_{a}, z_{a}\right)
\end{array}\right) .
$$


The time of the last collision, $t_{c}$, distributes randomly. Therefore we must average Eq. (4.6) over the distribution of $t_{c}$. If collisions are statistically independent, the probability of having had a collision with another atom in the time $d t_{c}$ between $t_{c}-d t_{c}$ and $t_{c}$ before the present time $t$ is

$$
\frac{1}{\tau_{c}} e^{-\left(t-t_{c}\right) / \tau_{c}} d t_{c}
$$

where $\tau_{c}$ is the mean free time for the atomic optical collisions. If $\mathrm{v}_{\mathrm{z}}^{\mathrm{a}}<0$, the atom is moving toward the wall so that the last collision could have occurred at any time in the past, i.e., $t_{c}$ could be $-\infty$. However, if $v_{z}^{a}>0$, the atom is moving away from the wall so that $t_{c}$ is limited by the distance to the wall $\mathrm{z}_{\mathrm{a}}$ and its thermal velocity $\mathrm{v}_{\mathrm{z}}^{\mathrm{a}}$; it becomes

$$
t_{c} \geqq t-\frac{z_{a}}{v_{z}^{a}} \quad, \quad\left(v_{z}^{a}>0\right) .
$$

Therefore, the probability, for an atom moving away from the wall, and having its last collision with another atom, is given by

$$
\int_{t-z_{a / v_{z}^{a}}^{a}}^{t} \frac{d \tau}{\tau_{c}} e^{-(t-\tau) / \tau_{c}}, \quad\left(v_{z}^{a}>0\right) .
$$

The complement of this is the probability of finding the atom, which was reflected from the wall, at the present position without any further collision. Such a wall collision must have occurred at

$$
t_{\omega}=t-z_{a} / v_{z}^{a}, \quad\left(v_{z}^{a}>0\right) .
$$

Therefore, the induced dipole moment averaged over 
the last collision is

$$
\begin{aligned}
\left\langle\vec{p}\left(\vec{r}_{a}(t), t\right)\right\rangle_{t_{x}} & =\left[U\left(-v_{z}^{a}\right) \int_{-\infty}^{t} \frac{d t_{c}}{\vec{\tau}_{c}} e^{-\left(t-t_{c}\right) / \tau_{c}} \cdot \delta_{t_{x}, t_{c}}+U\left(v_{z}^{a}\right)\left(e^{-z_{a} / v_{z}^{a} \tau_{c}} \cdot \delta_{t_{x}, t_{\omega}}\right.\right. \\
& \left.\left.+\int_{t-z_{a} / v_{z}^{a}}^{t} \frac{d t_{c}}{\tau_{c}} e^{-\left(t-t_{c}\right) / \tau_{c}} \delta_{t_{x}, t_{c}}\right)\right] \times \int_{t_{x}}^{t} d \tau \alpha(t-\tau) \vec{E}\left(\vec{r}_{a}(\tau), \tau\right)
\end{aligned}
$$

where $\delta t_{x}, t_{c}$ is the Kronecker delta and $U\left(v_{z}\right)$ is the Heaviside unit step function. In $\mathrm{Eq} .(4.8)$, since $\alpha(t-\tau)$ is non-local, we must consider an additional change of $\vec{E}$ at the atom due to its motion.

The field in Eq. (4.8) is the mean electric field at the moving atom. We express it in the Fourier integral form;

$$
\vec{E}\left(\vec{r}_{a}(t), t\right)=\frac{1}{2 \pi} \int_{-\infty}^{+\infty} d \omega e^{-i \omega t} \vec{E}\left(\vec{r}_{a}(t), \omega\right) .
$$

Using Eqs. (3.13) and (4.7), $\vec{E}\left(\vec{r}_{a}(t), \omega\right)$ can be written as

$$
\begin{aligned}
& \vec{E}\left(\vec{r}_{a}(t), \omega\right)=\iint d p d q\left(\frac{n_{0}}{\overrightarrow{|\vec{n}|}}\right)^{2} \vec{e}\left(\frac{n_{0}}{|\vec{n}|}, \frac{n_{0}}{|\vec{n}|} q_{1}, \omega\right) \exp \left\{i k\left(p x_{c}+q_{q} y_{c}+\frac{|\vec{n}|}{n_{0}} m^{\prime} z_{c}\right)-k \frac{n_{r} n_{i}}{|\vec{m}| n_{0}} \frac{z_{c}}{m^{\prime}}\right\} \times
\end{aligned}
$$

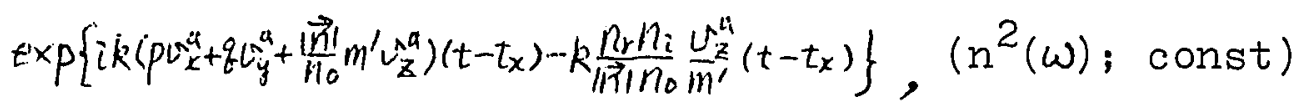

where $k$ is $k_{0} n_{0}$ with the refractive index of the glass as $n_{0} \cdot$ Thus, noting Eq. (4.5b) and assuming that the induced dipole has a single resonance at frequency $\omega_{c \ell}$, Eq. (4.8) becomes

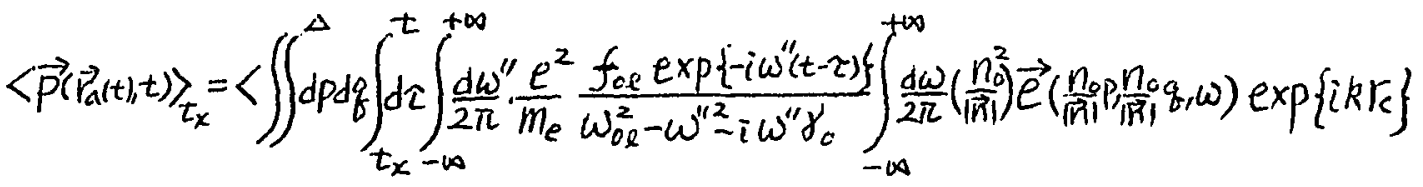

$$
\begin{aligned}
& \left.x \exp \left\{i k\{A+i B)\left(t-t_{x}\right)\right\} \exp \{i \omega \tau\}\right\rangle_{t_{x}},
\end{aligned}
$$

where $r_{c}=p x_{c}+\dot{q} y_{c}+\frac{|\vec{n}|}{n_{0}} m^{\prime} z_{c}+i \frac{n_{r} n_{i}}{|\vec{n}| n_{0}} \frac{z_{c}}{m^{\prime}}, A=p v_{x}^{a}+q v_{y}^{a}+\frac{|\vec{n}|}{n_{0}} m^{\prime} v_{z}^{a}$ and $B=\frac{n_{r} n_{i} v_{z}^{a}}{|\vec{n}| n_{0} m^{\prime}}$. 
Evaluating the integrals with respect to time $\tau$ as well as frequency $\omega^{\prime \prime}$ (with the help of contour integrals), Eq. (4.11) leads to

$$
\begin{aligned}
& \left\langle\vec{p}\left(\vec{r}_{a}(t), t\right)\right\rangle_{t_{x}}=-\frac{\hat{\epsilon}^{2}}{m_{e}} \frac{f_{o e}}{2 \omega_{i e}} \cdot \iint_{d p d q}^{\Delta} \int_{-\infty}^{+\infty} \frac{d \omega}{2 \pi}\left(\frac{n_{0}}{\mid \vec{n})}\right)^{2} \vec{e}\left(\frac{n_{0} p}{\mid \vec{n} p} p \frac{n_{c}}{|\vec{n}| q, \omega)} \exp \left\{i k r_{c}\right\} \times\right. \\
& \left\langle\exp \left\{i \omega t_{x}\right\}\left\{\frac{\exp \left\{-i \omega^{\prime}\left(t-t_{x}\right)\right\}-\exp \left\{-i \omega_{e}\left(t-t_{x}\right)\right\}}{\omega^{\prime}-\omega_{e}}-\frac{\exp \left\{-i \omega^{\prime}\left(t-t_{x}\right)\right\}-\exp \left\{i \omega_{e}\left(t-t_{x}\right)\right\}}{\omega^{\prime}+\omega_{e}}\right]\right\rangle_{\tau_{x}},
\end{aligned}
$$

where $\omega^{\prime}=\omega-K A-i k B$ and $\omega_{e}=\omega_{b e}-i \frac{\gamma_{0}}{2}$. For the optical region, we can neglect the off-resonance term (the second term of Eq. (4.12). Using Eq. (4.7), Eq. (4.12) can be written as

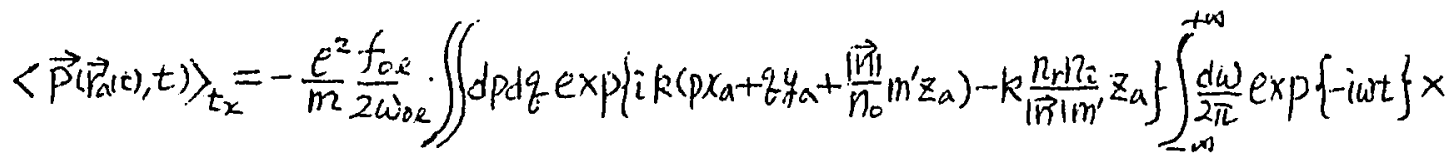

$$
\begin{aligned}
& \left(\frac{n_{0}}{|\vec{n}|}\right)^{2} \vec{e}\left(\frac{n_{0}}{|\vec{n}|}, \frac{n_{0}}{|\vec{n}|} q, \omega\right)\left\langle\frac{1-\exp \left\{i\left[\omega-\omega_{E[}-k A-i\left(k B-\frac{\gamma_{0}}{2}\right)\right]\left(t-t_{x}\right)\right.}{\omega-\omega_{0 R}-k A-i\left(k B-\gamma_{0 / 2}\right)}\right\rangle_{t_{x}} \cdot(4.13)
\end{aligned}
$$

If the incident electric field is a plane wave of a fixed mode, $\left(p_{0}, q_{0}, m_{0}\right), E q .(4.13)$ can now be written using Eq. $(3.47 a)$ as

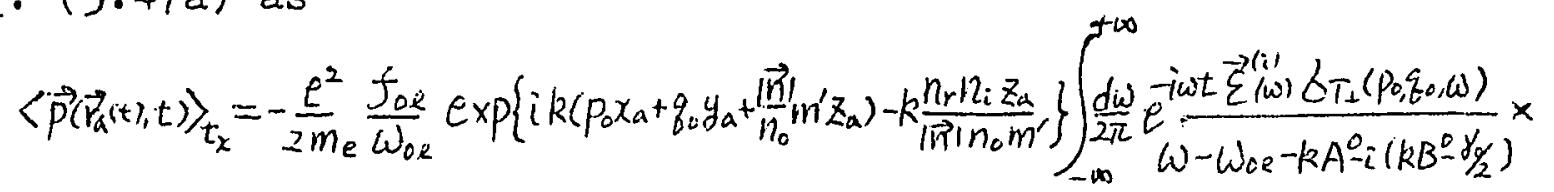

$$
\begin{aligned}
& \left\{\left[U\left(-v_{z}^{a}\right) \int_{-\infty}^{t} \frac{d t_{c}}{\tau_{i}} e^{-\left(t-t_{c}\right) / \tau_{c}} \delta_{t_{x}, t_{i}}+U\left(v_{z}^{a}\right)\left(e^{-\frac{z_{a}}{v_{z}^{a} \tau_{c}}} \delta t_{x, t_{i}}+\int_{t-\frac{z_{z}}{v_{z}^{a+1}}}^{t} \frac{d t_{c}}{\tau_{c}} e^{-\left(t-t_{i}\right) / \tau_{c}} \delta t_{x}, t_{c}\right)\right] \times\right. \\
& \left\{1-\exp \left\{\left[\left\{\left(\omega-\omega_{0 R}-k A^{2}-i\left(k B^{0}-\frac{y_{0}(a)}{2}\right)\right\}\left(t-t_{x}\right)\right\}\right],\right.\right.
\end{aligned}
$$

where the superscript " $O$ " on $A^{\circ}$ and $B^{\circ}$ refers to the fixed incident mode $\left(p_{0}, q_{0}, m_{0}\right)$. Evaluating the time integral above, we finally obtain 


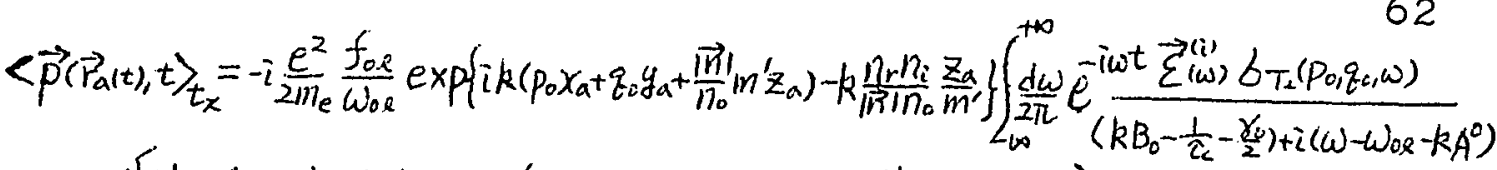

$$
\begin{aligned}
& x\left[U\left(-v_{z}^{a}\right)+U\left(v_{z}^{a}\right)\left(1-\exp \left\{i\left[\left(\omega-\omega_{c e}-k A^{c}\right)-i\left(k B^{0}-\frac{\gamma_{c}}{2}-\frac{1}{z_{c}}\right)\right] \frac{z_{a}}{v_{z}^{a}}\right\}\right] .\right.
\end{aligned}
$$

In Eq. (4.15), let us consider the expression, i.e.,

$$
U\left(-v_{z}^{a}\right)+U\left(v_{z}^{a}\right)\left(1-\exp \left\{i\left[\left(\omega \cdots \omega_{o r}-k A\right)-i\left(k B^{0}-\frac{p_{0}}{2}-\frac{1}{\tau_{c}}\right)\right] \frac{z_{a}}{v_{z}^{a}}\right\}\right) \text {. }
$$

The exponential term is due to the wall or surface effect. Only those atoms near the wall with positive $\hat{v}_{z}^{a}$ will be influenced by the presence of the wall and the wall effect will vanish for small $\dot{\mathrm{z}}_{\mathrm{z}}^{\mathrm{a}}$. For large $\dot{\mathrm{z}}_{\mathrm{z}}^{\mathrm{a}}$, namely, if

$$
\frac{Z_{a}}{v_{z}^{a}}\left(\frac{\gamma_{0}}{2}+\frac{1}{\tau_{c}}-k B^{c}\right) \ll 1,\left(\frac{\gamma_{c}}{2} \ll \frac{1}{\tau_{c}} \text { and } v_{z}^{a}>0\right)
$$

the exponential is nearly unity under a resonance condition, $\omega \simeq \omega_{i n}+k A^{0}$. As a result, there are no effective induced waves from those dipoles satisfying Eq. (4.16). We thus approximate the exponential term by a step function (for a treatment without this approximation see F. Takeda and M. Takeo, 1980);

$$
\exp \left\{i\left[\left(\omega^{\omega}-\omega_{c l}-k A^{0}\right)-i\left(k B^{0}-\frac{\gamma_{0}}{2}-\frac{1}{\tau_{c}}\right)\right] \frac{Z_{a}}{v_{z}^{a}}\right\}=U\left(v_{z}^{a}-v_{L}\right),
$$

where the upper limit (a truncation constant) may be determined from Eq. (4.16). Noting that $z_{a}$ is of the order of the penetration depth of the refracted light, $v_{\mathrm{I}}$ may be written as

$$
v_{L} \simeq\left(k_{0} \frac{n_{r} n_{i}}{|\vec{n}| \cos \theta_{0}^{\prime}}\right)^{-1} \tau_{L}^{-1}
$$

The induced electric dipole moment in $V_{ \pm}$is a microscopic field quantity. In order to derive the corresponding macroscopic polarization field, we define the probability density distribution function for the $\mathrm{Na}$ vapor atoms, consisting of $\mathrm{N}$ atoms in $\mathrm{V}_{ \pm}$, as

$$
\rho\left(\vec{x}_{1}, \cdots, \vec{x}_{a}, \cdots, \vec{x}_{N}\right)
$$


where $\vec{x}_{a}$ is $\left(\vec{r}_{a}, \vec{v}_{a}\right)$. Noting that the macroscopic polarization field is equal to the density of the dipole moments, we may write the macroscopic field for a single resonance line, $\ell$, as

$$
\vec{P}\left(\vec{r}_{,}\right)=\int\left(\sum_{a=1}^{N} \delta\left(\vec{r}-\vec{r}_{a}(t)\right)\left\langle\vec{p}\left(\vec{r}_{a}(t), t\right)\right\rangle_{t_{x}}\right) p\left(\vec{x}_{1}, \cdot, \vec{x}_{a}, \cdot \cdot \vec{x}_{N}\right) d \vec{x}_{1}, d \vec{x}_{a}, d \vec{X}_{N} .
$$

Since the atoms are identical, the probability distribution function is symmetric with respect to any permutation of the $\overrightarrow{\mathrm{x}} \mathrm{s}$, namely,

$$
\rho\left(\vec{x}_{1}, \cdots, \vec{x}_{n}, \vec{x}_{b}, \cdots, \vec{x}_{N}\right)=\rho\left(\vec{x}_{N}, \cdots, \vec{x}_{1}, \vec{x}_{a}, \cdots, \vec{x}_{b}\right) \text {. }
$$

Using this symmetry condition and carrying out the integrations over the $\vec{r}_{a}^{\prime}$ 's, we can rewrite Eq. (4.18) as

$$
\begin{aligned}
\vec{P}(\vec{r}, t) & =\int N\langle\vec{p}(\vec{r}, t)\rangle_{t_{x}} \rho\left(\vec{r}, \vec{v} ; \vec{x}_{2}, \cdots, \vec{x}_{N}\right) d \vec{v} d \vec{x}_{2}, \cdots, d \vec{x}_{N} \\
& =\int\langle\vec{p}(\vec{r}, t)\rangle_{t_{x}} f(\vec{r}, \vec{v}) d \vec{v},
\end{aligned}
$$

where $f(\vec{r}, \vec{v})$ is the "reduced one particle distribution function" (see the text of R. Balescu, 1975),

$$
f(\vec{r}, \vec{v})=N \int \rho\left(\vec{r}, \vec{v} ; \vec{x}_{2}, \cdots, \vec{x}_{N}\right) d \vec{x}_{2} \cdots d \vec{x}_{N} \text {. }
$$

We note that $f(\vec{r}, \vec{v})$ for the ideal gas is given by a Gaussian (Maxwellian) distribution,

$$
f(\vec{r}, \vec{v})=\frac{N}{V_{ \pm}}\left(\frac{2}{\sqrt{\pi}}\right)^{3} \frac{1}{\Delta v_{x}} \frac{1}{\Delta v_{y}} \cdot \frac{1}{\Delta v_{z}^{2}} \exp \left\{-\frac{4 v_{x}^{2}}{\Delta v_{x}^{2}}-\frac{4 v_{y}^{2}}{\Delta v_{y}^{2}}-\frac{4 v_{z}^{2}}{\Delta v_{z}^{2}}\right\} \text {. }
$$

Here $\mathrm{V}_{ \pm}$is the volume of the transition region and $\Delta \mathrm{v}_{\mathrm{x}}, \Delta \mathrm{v}_{\mathrm{y}}$ and $\Delta v_{z}$ are all equal to 


$$
\Delta V=2 \sqrt{2 \frac{k_{B} T}{M}}
$$

where $M$ is the mass of a $\mathrm{Na}$ atom, $k_{B}$ is the Boltzman constant and $T$ is the gas temperature.

The Fourier component of Eq. (4.19) is given by

$$
\begin{aligned}
\vec{P}(\vec{r}, \omega) & =-i \frac{e^{2}}{2 m_{e}} \frac{f_{0 l}}{\omega_{c e}}\left\{\int d^{3} v f(\vec{r}, \vec{v}) \frac{\left[U\left(-v_{z}\right)+U\left(v_{z}\right)\left(1-U\left(v_{z}-v_{L}\right)\right)\right]}{\left(k B^{0}-\frac{1}{\tau_{L}}-\frac{\gamma_{c}}{2}\right)+i\left(\omega-\omega_{c e}-k A^{0}\right)}\right. \\
& \times \vec{\varepsilon}^{(i)}(\omega) b_{T_{L}}\left(p_{0}, q_{0}, \omega\right) \exp \left\{i k\left(p_{0} x+q_{0} y+\frac{|\vec{n}|}{n_{0}} m^{\prime} z\right)-k \frac{n_{r} n_{i}}{|\vec{n}| n_{0}} \frac{z}{m^{\prime}}\right\} .
\end{aligned}
$$

Using the relation,

$$
\vec{P}(\vec{r}, \omega)=\eta_{l}\left(p_{0}, q_{0}, \omega\right) \vec{E}(\vec{r}, \omega),
$$

we find that the electric susceptibility $\eta_{l}$, for a single resonance line $\ell$, is

$$
\eta_{l}\left(p_{c}, q_{c}, \omega\right)=-i \frac{e^{2}}{2 m_{e}} \frac{f_{0, \ell}}{\omega_{0, e}} \int d v f\left(\vec{r}_{1}, \vec{v}\right) \frac{\left[U\left(v_{z}\right)+U\left(v_{z}\right)\left(1-U\left(U_{z}-v_{L}\right)\right)\right]}{\left(k B^{0}-\frac{1}{\tau_{c}}-\frac{\gamma_{i}}{2}\right)+i\left(\omega-\omega_{c z}-k A^{\circ}\right)} .
$$

In the following, we specify the plane of incidence (the $x-z$ plane) by setting $\phi_{0}=0$ (see section 3 in Chapter III). $A^{\circ}$ and $B^{\circ}$ can then be written as

$$
k A^{0}=k \sin \theta_{0} v_{x}+k_{0}|\vec{n}| \cos \theta_{0}^{\prime} v_{z}
$$

and

$$
k B^{\circ}=k_{0} \frac{n_{r} n_{i}}{|\vec{n}| \cos \theta_{0}^{\prime}} v_{z},
$$

where the angles, $\theta_{0}$ and $\theta_{0}^{\prime}$, are the incident and the refracted angle respectively. For later convenience in calculating numerically the line shape of the reflected light, we express $A^{0}$ and $B^{0}$ in terms of $\theta 0_{0}, n_{r}, n_{i}$ and $n_{0}$. To do this, we take the square root of both sides of $\mathrm{Eq} \cdot(3.40)$. We then find

$$
|\vec{n}| m^{\prime}+i \frac{n_{r} n_{i}}{|\vec{n}| m^{\prime}}=A\left(\theta_{0}\right)+i B\left(\theta_{0}\right), \quad\left(m^{\prime}=\cos \theta_{0}^{\prime}\right)
$$


where $A\left(\theta_{0}\right)$ and $B\left(\theta_{0}\right)$ are

$$
\left.\begin{array}{l}
A\left(\theta_{0}\right) \\
B\left(\theta_{0}\right)
\end{array}\right\}=\frac{1}{\sqrt{2}}\left\{ \pm\left(n_{r}^{2}-n_{i}^{2}-n_{0}^{2} \sin ^{2} \theta_{0}\right)+\left[\left(n_{r}^{2}-n_{i}^{2}-n_{0}^{2} \sin ^{2} \theta_{0}\right)^{2}+4\left(n_{r} n_{i}\right)^{2}\right]^{1 / 2}\right\}^{1 / 2} .
$$

Thus Eqs. (4.24a) and $(4.24 \mathrm{~b})$ can be written as

$$
k A^{0}=k \sin \theta_{0} v_{x}+k_{0} A\left(\theta_{0}\right) v_{z}, \quad\left(k=n_{0} k_{0}\right),
$$

and

$$
k B^{0}=k_{0} B\left(\theta_{0}\right) i_{z}, \quad\left(k_{0}=\frac{2 \pi}{\lambda}\right) .
$$

In order to compare our results with the experimentally observed line shapes, we express the electric susceptibility in terms of the frequency $\nu$ instead of the angular frequency $\omega$. The real and the imaginary part of $\eta_{q}\left(\theta_{0}, \nu\right)$ can be written as

$$
\begin{aligned}
& \eta_{l}^{r}\left(\theta_{0}, v\right)=-\frac{e^{2}}{2 m e} \frac{f_{\Delta \varepsilon}}{(2 \pi)^{2}} \cdot \frac{1}{V_{c e}} \cdot \frac{N}{V_{ \pm}} \int_{-\infty}^{+\infty} d v_{x} \frac{2}{\sqrt{\pi}} \cdot \frac{1}{\Delta v} \exp \left\{-\frac{4 v_{x}^{2}}{\Delta v^{2}}\right\} \int_{-\infty}^{v_{L}} d v_{z} \frac{2}{\sqrt{\pi}} \frac{1}{\Delta v} \exp \left\{-\frac{4 v_{z}^{2}}{\Delta v^{2}}\right\} \times \\
& \frac{\nu-\nu_{e}}{\left(\nu-\nu_{e}\right)^{2}+\left(B\left(\theta_{0}\right) V_{z} / \lambda-1 / 2 \pi \tau_{c}-\Gamma_{0} / 2\right)^{2}},\left(\Gamma_{0}=\frac{\gamma_{c}}{2 \pi}\right),(4.27 a) \\
& \eta_{l}^{i}\left(\theta_{0}, \nu\right)=-\frac{e^{2}}{2 m} \frac{f_{0 Q}}{(2 \pi)^{2}} \frac{1}{v_{c} e} \frac{V}{V_{I}} \int_{-\infty}^{+\infty} d v_{x} \frac{2}{\sqrt{\pi}} \frac{1}{\Delta v} \exp \left\{-\frac{4 v_{x}^{2}}{\Delta v^{2}}\right\} \int_{-\infty}^{v_{i}} d v_{z} \frac{2}{\sqrt{\pi}} \frac{1}{\Delta v} \exp \left\{-\frac{4 v_{z}^{2}}{\Delta v^{2}}\right\} x \\
& \frac{B\left(\theta_{0}\right) v_{3}^{\infty} / \lambda-1 / 2 \pi \tau_{c}-\Gamma_{0} / 2}{\left(\nu-\nu_{l}\right)^{2}+\left(B\left(\theta_{0}\right) U_{z} / \lambda-1 / 2 \pi \tau_{c}-\Gamma_{0} / 2\right)^{2}} \text {. }
\end{aligned}
$$

Here the upper limit for the $\dot{v}_{z}$ integration is (see Eq. $(4.17))$

$$
v_{L} \approx \frac{\lambda}{B\left(\theta_{0}\right)} \frac{1}{2 \pi \tau_{c}} \text {. }
$$

Further, $V_{l}$ is the Doppler shifted resonance frequency given by

$$
\nu_{l}=\nu_{0 l}+\nu n_{0} \frac{V_{x}}{c} \sin \theta_{0}+\nu A\left(\theta_{0}\right) \frac{V_{z}}{c} \text {. }
$$

This can be approximated for the present case, where the 
incident frequency $\mathcal{V}$ is near the natural resonance frequency $Y_{0 e}$, as

$$
\nu_{l}=\nu_{i l}\left(1+n_{0} \frac{V_{x}}{c} \sin \theta_{0}+A\left(\theta_{0}\right) \frac{V_{z}}{c}\right) \text {. }
$$

The electric susceptibility $\eta_{\ell}\left(\theta_{0}, \mathcal{V}\right)$ is related to the complex refractive index $h(\omega)$ (for the single resonance line, $l$ ) through the following relation,

$$
n\left(\theta_{0}, \nu\right)=\sqrt{1+4 \pi \eta\left(\theta_{0}, \nu\right)} \text {. }
$$

Since $n=n^{r}+i n^{i}$ and $\eta_{e}=\eta_{e}^{r}+i \eta_{e}^{i}$, from $E q$. (4.30) we obtain

$$
n_{r}^{2}-n_{i}^{2}=1+4 \pi \eta_{l}^{r}
$$

and

$$
n^{r} n^{i}=2 \pi \eta_{e}^{i}
$$

We can then express $A\left(\theta_{0}\right)$ and $B\left(\theta_{0}\right)$ in terms of $\eta_{l}^{r}\left(\theta_{0}, \nu\right)$ and $\eta_{x}^{i}\left(\theta_{0}, \mathcal{\nu}\right)$. They are

$$
\left.\begin{array}{l}
A\left(\theta_{0}\right) \\
B\left(\theta_{0}\right)
\end{array}\right\}=\frac{1}{\sqrt{2}}\left\{ \pm\left(1+4 \pi \eta_{l}^{r}-\eta_{0}^{2} \sin ^{2} \theta_{0}\right)+\left[\left(1+4 \pi \eta_{l}^{r}-n_{0}^{2} \sin ^{2} \theta_{0}\right)^{2}+4 \cdot\left(2 \pi \eta_{l}^{i}\right)^{2}\right]^{1 / 2}\right\}^{1 / 2} .
$$

Eqs. (4.27a) and $(4.27 \mathrm{~b})$ can be solved on a digital computer by using an iteration technique (see Appendix A).

In order to see more clearly the behavior of the electric susceptibility, we change the integration variables in Eqs. (4.27a) and (4.27b) using the following transformations:

$$
\begin{aligned}
& F_{e}=\nu-\nu_{0 e}, \\
& x_{e}=\nu_{c e} n_{0} \sin \theta_{0} \frac{\nu_{x}}{c}
\end{aligned}
$$

and

$$
Z_{l}=V_{0 e} A\left(\theta_{0}\right) \frac{V_{z}}{C} \text {. }
$$


Then Eq. (4.27a) can be written as

$$
\begin{aligned}
& \eta_{l}^{r}\left(\theta_{0}, F_{l}\right)=-\frac{e^{2}}{2 m_{e}} \frac{f_{e l}}{(2 \pi)^{2}} \frac{1}{\nu_{0 R}} \frac{N}{V_{ \pm}} \int_{-\infty}^{+\infty} d x_{l} \frac{2}{\sqrt{\pi}} \cdot \frac{1}{\Delta x_{l}} \exp \left\{-\frac{4 x_{l}^{2}}{\Delta x_{l}^{2}}\right\} \cdot \int_{-\infty}^{L} d Z_{l}, \frac{2}{\sqrt{\pi}} \cdot \frac{1}{\Delta Z_{l}} \exp \left\{-\frac{4 Z_{l}^{2}}{\Delta Z_{l}^{2}}\right\} x \\
& \frac{\bar{H}_{l}-X_{l}-Z_{l}}{\left(F_{l}-X_{l}-Z_{l}\right)^{2}+\left(B\left(\theta_{0}\right) \cdot Z_{l} / A\left(\theta_{0}\right)-\Gamma_{0} / 2-1 / 2 \pi \tau_{c}\right)^{2}} \text {, }
\end{aligned}
$$

where $\Delta \mathrm{x}_{\ell}=\nu_{0 \ell} \mathrm{n}_{0} \sin \theta_{0} \frac{\Delta U}{\mathrm{C}}, \Delta \mathrm{z}_{\ell}=\nu_{0 \ell} \mathrm{A}\left(\theta_{0}\right) \frac{\Delta V}{\mathrm{C}}$ and $L_{s}=\frac{A\left(\theta_{c}\right)}{B\left(\theta_{0}\right)} \cdot \frac{1}{2 \pi \tau_{c}}$. Similarly, Eq. (4.27b) becomes

$$
\begin{aligned}
\eta_{l}^{i}\left(\theta_{0}, F_{l}\right)=-\frac{e^{2}}{2 m_{e}} \cdot \frac{f_{0 l}}{(2 \pi)^{2}} \cdot \frac{1}{\nu_{0 l}} \cdot \frac{N}{V_{I}} \int_{-\infty}^{+\infty} d X_{l} \frac{2}{\sqrt{\pi}} \frac{1}{\Delta X_{l}} \exp \left\{-\frac{4 X_{l}^{2}}{\Delta X_{l}^{2}}\right\} \int_{-\infty}^{L} d Z_{l} \frac{2}{\sqrt{\pi}} \cdot \frac{1}{\Delta Z_{l}} \exp \left\{-\frac{4 Z_{l}^{2}}{\Delta Z_{l}^{2}}\right\} x \\
\frac{\left(B\left(\theta_{0}\right) Z_{l} / A\left(\theta_{0}\right)-\Gamma_{0} / 2-1 / 2 \pi \tau_{l}\right)}{\left(F_{l}-X_{l}-Z_{l}\right)^{2}+\left(B\left(\theta_{0}\right) Z_{l} / A\left(\theta_{0}\right)-\Gamma_{0} / 2-1 / 2 \pi \tau_{c}\right)^{2}} \cdot(4.34)
\end{aligned}
$$

For the case where the angle of incidence is normal $\left(\theta_{0}=0\right)$, $\mathrm{X}_{\ell}=0$ and there is no integration over $\mathrm{X}_{\ell}$. In this case, we have a partial absence of Doppler broadening in $\eta_{l}^{r}\left(\theta_{0}, F_{l}\right)$ and $\eta_{l}^{i}\left(\theta_{0}, F_{l}\right)$ due to the restricted selection of dipoles, i.e., the restricted upper limit on $Z_{l}$. When the incident angle increases, the Doppler broadening due to the integral over $\mathrm{x}_{\ell}$ becomes large and the contribution from the $\mathrm{Z}_{\ell}$ integration becomes less important. Using the expressions for the reflectivity, Eqs. (3.48c) and (3.49c), for an incident monochromatic plane wave, the numerically calculated line shapes of the reflected light are similar to those observed experimentally (see Appendix A). 
We recall that the damped harmonic oscillator in our model has two isolated resonances, $l=1$ and $l=2$. In this case the electric susceptibility $\eta\left(\theta_{0}, \nu\right)$ can be written as a sum,

$$
\eta\left(\theta_{0}, \nu\right)=\sum_{l=1}^{2} \eta_{l}\left(\theta_{0}, F_{l}\right) \text {. }
$$

In the next section, in order to compare our results with the experimentally observed line shapes, we derive expressions for the line shape of the reflected light when incident light with a relatively narrow line width sweeps across the two resonances, $l=1$ and $l=2$.

\section{THE LINE SHAPE \\ OF THE REFLECTED LIGHT}

We assume that the spectral distribution $\vec{\varepsilon}^{(i)}(\nu)$ of the incident light is Gaussian, with a maximum amplitude $\varepsilon_{0}$. Then

$$
\vec{\varepsilon}^{(i)}(\nu)=\hat{\lambda} \varepsilon_{0} \exp \left\{-\frac{2\left(\nu-\nu^{(i)}\right)^{2}}{\Delta \nu^{2}}\right\}
$$

where $\hat{\lambda}$ is the polarization unit vector and $\nu^{(i)}$ is the central frequency. For the case of perpendicular polarization $\hat{\lambda}_{1}$, (see section 3 of Chapter III), the reflectivity can be written as

$$
R_{\perp}\left(\theta_{0}, \nu^{(i)}\right)=\frac{\left|\int_{-\infty}^{+\infty} d \nu \vec{\varepsilon}^{(i)}(\nu) b_{R_{L}}\left(\theta_{0}, \nu\right)\right|^{2}}{\left|\int_{-\infty}^{+\infty} d \nu \vec{\varepsilon}^{(i)}(\nu)\right|^{2}} .
$$

Here we note from Eq. (3.45b) that the reflection amplitude, $b_{R_{\perp}}\left(\theta_{0}, \nu\right)$, is given as 


$$
b_{R_{\perp}}\left(-\theta_{0}, \nu\right)=\frac{n_{0} \cos \theta_{0}-\sqrt{n^{2}(\nu)-n_{0}^{2} \sin ^{2} \theta_{0}}}{n_{0} \cos \theta_{0}+\sqrt{n^{2}(\nu)-n_{0}^{2} \sin ^{2} \theta_{0}}} .
$$

Using Eqs. (3.40) and (4.25a), we can express Eq. (4.38) in terms of the real and imaginary parts of the susceptibility, $\eta^{r}\left(\theta_{0}, \nu\right)$ and $\eta^{(i)}\left(\theta_{0}, \nu\right)$.

$$
b_{R_{\perp}}\left(\theta_{c}, \nu\right)=\frac{n_{0} \cos \theta_{0}-A\left(\theta_{c}, \nu\right)-i B\left(\theta_{0}, \nu\right)}{n_{0} \cos \theta_{0}+A\left(\theta_{c}, \nu\right)+i B\left(\theta_{0}, \nu\right)}
$$

where

$$
\left.\left.\begin{array}{l}
A\left(\theta_{0}, \nu\right) \\
B\left(\theta_{0, \nu}\right)
\end{array}\right\}=\frac{1}{\sqrt{2}}\left\{ \pm\left(1+4 \pi \eta^{2}\left(\theta_{0}, \nu\right)-n_{0}^{2} \sin ^{2} \theta_{0}\right)+\left[\left(1+4 \pi \eta^{2}\left(\theta_{c}, \nu\right)-n_{0}^{2} \sin ^{2} \theta_{0}\right)^{2}+4\left(2 \pi \eta^{i}\left(\theta_{c} \nu\right)\right)\right)^{2}\right]^{\frac{1}{2}}\right\}^{\frac{1}{2}} .
$$

For incident light with parallel polarization, we obtain the reflectivity using Eq. (3.49b),

$$
R_{1 /}\left(\theta_{0}, \nu^{(i)}\right)=\frac{\left|\int_{-\infty}^{+\infty} d \nu \vec{x}^{(i)}(\nu) \pi_{R /}\left(\theta_{0}, \nu\right)\right|^{2}}{\left|\int_{-\infty}^{+\infty} d \nu \vec{x}^{(i)}(\nu)\right|^{2}},
$$

where the functional form of $\vec{म}^{(i)}$ is also assumed to be Gaussian,

$$
\vec{X}^{(i)}(\nu)=\hat{\lambda}_{1} X_{0} \exp \left\{-\frac{2\left(\nu-\mathcal{\nu}^{(i)}\right)^{2}}{\Delta \nu^{2}}\right\}
$$

We can also write $\pi_{R_{11}}\left(\theta_{0}, \nu\right)$ in terms of $\eta^{r}\left(\theta_{0}, \nu\right)$ and $\eta^{i}\left(\theta_{0}, \nu\right)$ as

$$
\pi_{R_{1}}=\frac{\left(1+4 \pi \eta^{r}\left(\theta_{0}, \nu\right)\right) \cos \theta_{0}+i 4 \pi \eta^{i}\left(\theta_{0}, \nu\right) \cos \theta_{0}-n_{0} A\left(\theta_{0}, \nu\right)-i \eta_{0} B\left(\theta_{0}, \nu\right)}{\left(1+4 \pi \eta^{r}\left(\theta_{0}, \nu\right)\right) \cos \theta_{0}+i 4 \pi \eta^{i}\left(\theta_{0}, \nu\right) \cos \theta_{0}+n_{0} A\left(\theta_{0}, \nu\right)+n_{0} i B\left(\theta_{0}, \nu\right)},
$$


where $A\left(\theta_{0}, \mathcal{\nu}\right)$ and $B\left(\theta_{0}, \nu\right)$ are given by Eq. (4.39b). line shapes can be numerically calculated from Eqs. (4.37) and (4.40a) with the help of a digital computer (see Appendix A). 


\section{CHAPTER $V$}

\section{APPLICATION OF SATURATION SPECTROSCOPY TO SURFACE INVESTIGATION}

\section{INTRODUCTION}

When a tunable laser with a narrow line width is used as a light source, it can selectively excite atoms whose Doppler shifted resonnace frequencies are within the narrow Iine width. The atoms with these particular thermal velocities are excited and temporarily removed from those atoms in the ground state. If the beam is sufficiently intense so that the rate of excitation is faster than that of de-excitation, these excited atoms produce a hole in the velocity distribution of the atoms in the ground state. This phenomenon has been referred to as "hole burning" (W. R. Bennett, Jr., 1962). The shape of this hole burned into the distrubution can be observed by scanning a second and weak tunable laser beam over the entire Doppler profile. This spectroscopic technique is know as "saturation spectroscopy" (see a review article of W. Lange, J. Iuther and A. Steudel, 1974, and T. W. Hänsh, A. I. Schawlow and G. W. Series, 1979).

Since the line shape of the reflected beam probing this "hole" is nearly free from Doppler broadening, we may be able to use this line shape for surface investigations. 
The line shape of interest is that of light reflected from an environmentally related model interface where interactions between a solid wall and gaseous atoms (or molecules) create optical properties different from that of the bulk gas. Interaction potentials between a solid surface and gaseous atoms (or molecules) have yet to be studied, both experimentally and theoretically (see A. Shih and V. A. Parsegian, 1975; also review articles by T. Takaishi, 1975, and by S. Nir, 1976). For this purpose, we will investigate the experimental feasibility of the tunable dye laser as a new surface probe.

\section{REFLECTION AT A SOLID-GAS INTERFACE WITHOUT INTERACTIONS}

In order to investigate the validity of saturation spectroscopy for surface investigations, we use the previously studied model interface of glass and $\mathrm{Na}$ vapor. For this purpose, we split an intense laser beam with a narrow line width into a strong and a weak beam by a beam splitter. Then let both the strong and the weak beam be incident on the solid-gas interface at the angles, $\theta_{10}$ and $\theta_{20}$, respectively. When $\theta_{10}$ and $\theta_{20}$ are larger than the critical angle of the system, the refracted angles are about 89 degrees. If the beams are incident from opposite sides of the normal, the two refracted beams are propagating nearly antiparallel to each other. On the other hand, if the beams are incident on the same side, the refracted beams result in nearly para- 
IleI propagation (see Fig. 5.I).

Suppose that there are $\mathrm{N}_{0}^{0}$ atoms in the ground state "O" per unit volume and $N_{\ell}^{\circ}$ atoms in the excited state "l" per unit volume. In the absence of incident radiation, collisions maintain their thermal equilibrium distributions. We now assume that the intense laser beam is tuned to the resonance line " $l_{0}$ ". For the moment, we assume that the weak beam is cut off. Then the radiative transition between the atomic states due to the strong incident beam with an incident frequency $\nu$ will be induced at a rate that is not negligible compared with the collision rate $\tau^{-1}$. Thus the gas is no longer in thermal equilibrium, and a new equilibri -um between the two states "o" and " $\ell$ " must be set up. Under this new equilibrium, the probability, $W_{o r}^{\prime}$, of finding a moving atom near the wall, which was initially in the "o" state, in the excited state " $l$ " in time $\tau$ may be written as (R. Karplus and J. Schwinger, 1948 and the text of C. H. Townes and A. I. Schawlow, 1955),

$$
W_{o l}^{\prime}=\frac{G^{2}}{2} \frac{\left(1 / 2 \pi \tau_{c}\right)^{2}}{\left(\nu-\nu_{i l}^{\prime}\right)^{2}+\left(1 / 2 \pi \tau_{c}\right)^{2}\left(1+G^{2}\right)},
$$

where $G^{2}=e^{2} f_{o e}|\vec{E}|^{2} \tau_{c} \tau /\left(4 m_{e} \nu_{i e} h\right)$, with the electron mass $m_{e}$, Planck's constant $h$, and $\nu_{0 r}^{\prime}=\nu_{0,}\left(I+n_{0} \frac{V_{x}}{c} \sin \theta_{10}\right)$ (see Eq. (4.29)). $\tau_{c}$ is the mean free time for the atomic optical collisions. $\vec{E}$ is the mean electric field at the atom. Recall that when the incident angle is large, a significant contribution to the reflected light comes from those atoms which are moving parallel to the wall. Hereaf- 


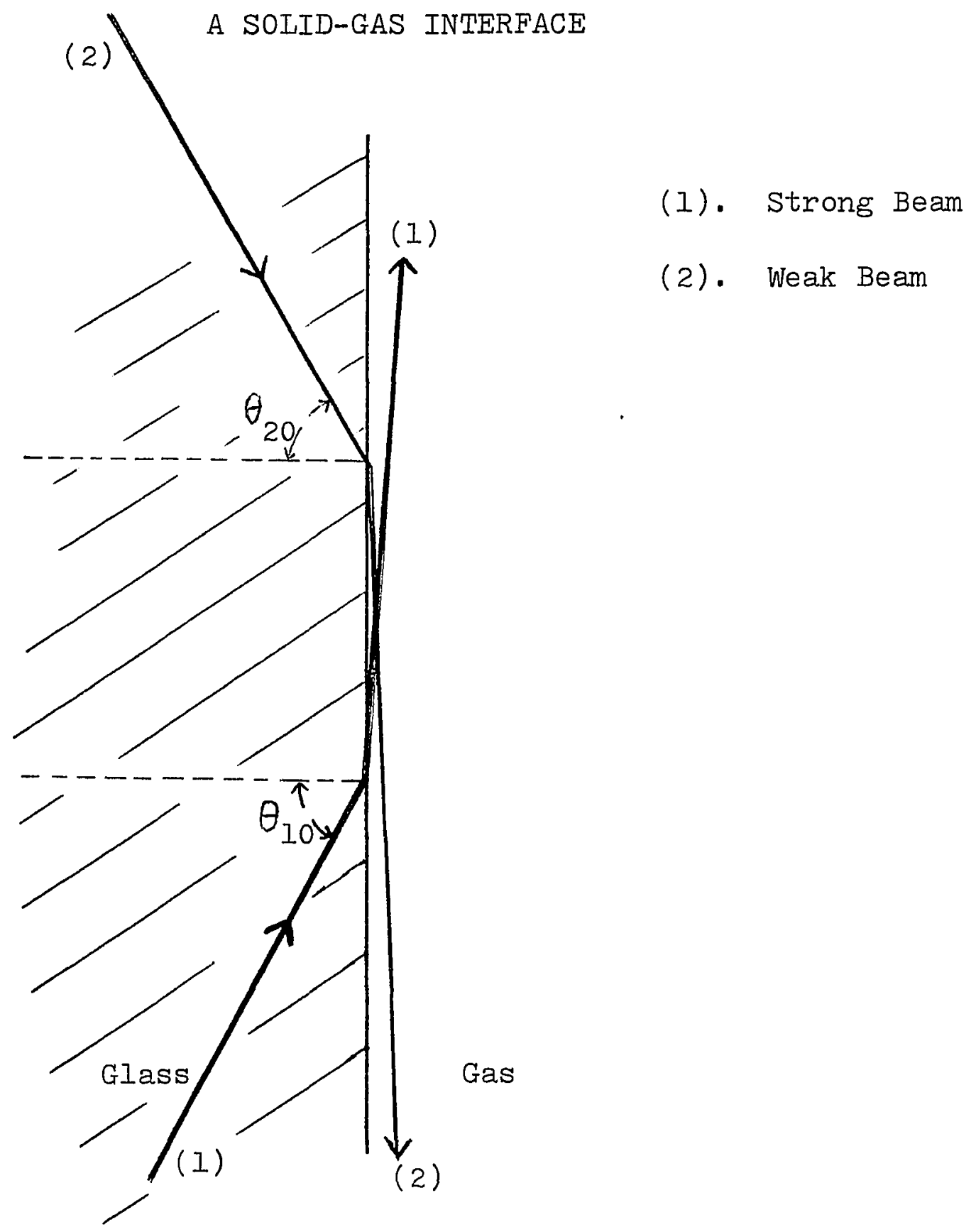

Fig. 5.1 
ter we suppress the subscript " $x$ " on the parallel volocity component $v_{x}$.

The strong beam can resonantly interact with the atoms if their Doppler shifted resonance frequencies are within the laser's narrow line width $\delta \nu^{(i)}$. Thus, denoting by $\mathrm{v}^{0}$ the velocity of the atom whose Doppler shifted resonance frequency is equal to the incident frequency, $\nu^{(i)}$, at the intensity peak, the restriction on $\mathrm{v}$ for the resonance can be expressed as

$$
v^{0}-\frac{1}{2} \delta v^{0} \leq v \leq v^{0}+\frac{1}{2} \delta v^{\circ},
$$

where $V^{0}=c\left(\nu^{(i)}-\nu_{o R}\right) / \nu_{o s}$ and $\delta V^{0}=c \delta \nu^{(i)} / \nu_{0 e}$.

Using the unit step function, $U(\nu)$, the probability, Wor, of finding the atom in the " $l$ " state, which is selectively excited from the ground state "o" in time $\tau$ by the strong beam, may be written as

$$
W_{c \Omega}(\theta, 0, \nu, v)=\left[U\left(\nu-\nu^{(i)}+\frac{1}{2} \delta \nu^{(i)}\right)-U\left(\nu-\nu^{(i)}-\frac{1}{2} \delta \nu^{(i)}\right)\right] \frac{G^{2}}{2} \frac{\left(1 / 2 \pi \tau_{c}\right)^{2}}{\left(\nu-\nu_{c l}^{\prime}\right)^{2}+\left(1 / 2 \pi \tau_{c}\right)^{2}\left(1+G^{2}\right)} \cdot
$$

This transition creates a hole in the velocity distribution of atoms in the ground state. Thus the new equilibrium velo -city distribution function with this "burned hole" may be written as

$$
f_{0}(v)=f_{0}^{0}(v)\left(1-W_{0}\right),
$$

where $f_{0}^{\circ}(v)$ is the reduced one particle distribution func -tion similar to Eq. (4.25),

$$
f_{0}^{0}(v)=\frac{N}{V_{ \pm}} \frac{2}{\sqrt{\pi}} \frac{1}{\Delta v} \exp \left\{-\frac{4 v^{2}}{\Delta v^{2}}\right\}
$$

and $\mathrm{N}$ is the average number of atoms in $\mathrm{V} \pm$. 
Here we note that Eq. (5.4) does not include another possible source to increase the ground state population in the hole. Because of gas-kinetic collisions, the velocities of the atoms will be redistributed. Thus the number of atoms in the ground state, all of which are in the narrow velocity distribution corresponding to the hole, will change. However, if the optical excitation rate, $P_{0 \rightarrow \ell}\left(=P_{l \rightarrow 0}\right)$, is large, we can ignore this change. This can be justified in the following way. Consider the rate equation for the ground state population $N_{0}$. Atoms are transferred by collisions from the ground to the excited state at the probability rate $\tau_{o x}^{-1}$, or conversely at the rate $\tau_{i 0}^{-1}$. For the gas in equilibrium with no incident radiation, we have

$$
\frac{N_{e}^{c}}{\tau_{l o}}-\frac{N_{0}^{c}}{\tau_{0 e}}=0
$$

Then, in the hole, the rate equation of the ground state with incident radiation can be written as

$$
\frac{d N_{0}}{d t}=\frac{\tilde{N}_{i}}{\tau_{k}}+\frac{N_{l}}{\tau_{l c}}-\frac{N_{0}}{\tau_{b l}}+N_{l} P_{l \rightarrow 0}-N_{0} P_{0 \rightarrow \ell,} \quad\left(P_{l \rightarrow 0}=P_{0 \rightarrow \ell}\right),
$$

where $\tau_{k}^{-1}$ is the probability rate of transferring atoms from outside to inside the hole by gas-kinetic collisions. $\tilde{\mathrm{N}}_{\mathrm{O}}$ is related to the population in the ground state outside the hole, which can only come into the hole by the collisions within time $\tau_{k}$. In steady state, $\widetilde{N}_{0} \simeq \mathbb{N}_{l}$ and we obtain

$$
N_{l}\left(1 / \hat{\tau}_{k}+1 / \hat{\tau}_{l}+P_{l \rightarrow 0}\right)-N_{0}\left(1 / \tau_{a l}+P_{0 \rightarrow l}\right)=0 .
$$

If the optical excitation is large so that $P_{l \rightarrow 0} \gg \tau_{k}^{-1}$, the 
steady state condition Eq. (5.7) gives the "burned hole" predicted by Eq. (5.4), under the same condition, influences from the atoms in the region outside of $V_{ \pm}$on the population change in $\mathrm{N}_{0}$ can be ignored.

Using Eq. (5.4), the macroscopic polarization vector field $\vec{P}(\vec{r}, \nu)$, which is induced by the weak probing beam with the incident angle $\theta_{20}$, can be expressed by an expression similar to Eq. (4.22). The electric susceptibility $\eta_{e}\left(\theta_{20}, \nu\right)$ is then given by an expression similar to Eq. (4.23). The real and the imaginary part are given by (see Eqs. $(4.27 \mathrm{a})$ and $(4.27 \mathrm{~b})$ );

$$
\begin{aligned}
& \eta_{e}^{r}\left(\theta_{20}, \nu\right)=-\frac{e^{2}}{2 m_{e}} \cdot \frac{f_{0 \ell}(2 \pi)^{2}}{\nu_{3 e}} \cdot \frac{N}{V_{ \pm}} \int_{-\infty}^{+\infty} d v \frac{z}{\sqrt{\pi}} \frac{1}{\Delta v} \exp \left\{-\frac{4 v^{2}}{\Delta v^{2}}\right\}\left[1-W_{i e}\left(v, \nu, \theta_{10}\right)\right] \times \\
& \frac{\nu-\nu_{c e}^{\prime \prime}}{\left(\nu-\nu_{0 . \ell}^{\prime \prime}\right)^{2}+\left(1 / 2 \pi \tau_{c}+P_{0} / 2\right)^{2}}
\end{aligned}
$$

and

$$
\begin{aligned}
\eta_{e}^{i}\left(\theta_{20}, \nu\right)= & -\frac{e^{2}}{2 m_{e}} \frac{f_{c e}}{(2 \pi)^{2}} \frac{1}{\nu_{c e}} \frac{N}{V_{ \pm}} \int_{-\infty}^{+\infty} d v \frac{z}{\sqrt{\pi}} \frac{1}{\Delta v} \exp \left\{-\frac{4 v^{2}}{\Delta v^{2}}\right\}\left[1-W_{c R}\left(v, \nu, \theta_{10}\right)\right] \times \\
& \frac{-\left(1 / 2 \pi \tau_{c}+\Gamma_{0} / 2\right)}{\left(\nu-\nu_{c l}^{\prime \prime}\right)^{2}+\left(1 / 2 \pi \tau_{c}+\Gamma_{0} / 2\right)^{2}},
\end{aligned}
$$

where $\nu_{c e}^{\prime \prime}=\nu_{c e}\left(1+n_{0} \frac{U}{C} \sin \theta_{20}\right)$ and $\Delta v=2 \sqrt{2 k_{B} T / M}$.

In order to numerically calculate the line shape of the reflected weak beam, we assume that the spectral distribution of the incident weak and the strong beam is Gaussian (like Eq. (4.36) where $\Delta \nu=\delta \nu^{(i)}$ ), and that $\delta \mathcal{V}^{(i)}$ is the order of $\tau_{c}^{-1}$. The two beams are incident from opposite sides of the normal with $\theta_{10}=\theta_{20}=\theta_{0}$, 
and the Doppler shifted resonance frequency $V_{\text {be }}^{\prime}$ in Woe is $\nu_{o l}^{\prime}=\nu_{o l}\left(1-n_{0} \frac{U}{C} \sin \theta_{0}\right)$. We now substitute the above $\eta_{l}^{r}\left(\theta_{0}, \nu\right)$ and $\eta_{l}^{i}\left(\theta_{0}, \nu\right)$ into Eq. $(4.31)$, and then use the line shape expressions, Eqs. (4.37) and (4.40a) from section 3 of Chapter IV to carry out the calculation.

We scan the incident frequency, $\nu^{(i)}$, of the incident beam over the entire Doppler profile. Since the propagation directions of the two refracted beams are anti -parallel along the surface, the weak beam can detect the hole burned out by the strong beam only when the incident frequency $\nu^{(i)}$ is tuned to the unshifted natural resonance frequency $\nu_{\text {or }}$. Thus the atoms which are responsible for the hole detected by the weak beam are almost at rest. Therefore the line shape of the reflected weak beam from the "burned hole" is nearly free from Doppler broadening. When the saturation effect is relatively weak, the entire pressure broadened profile may be obtain -ed. A result of the above model calculation is shown in Fig. 5.2. We note that a profile of the "burned hole" may also be obtained by scanning the weak beam over the entire Doppler broadened profile, and that the line width of the weak beam is not an important factor in detecting the "burned hole" (see also Appendix B). WITH INTERACTIONS 
〈REFLECTIVITY

$(\star 10 M H Z)$
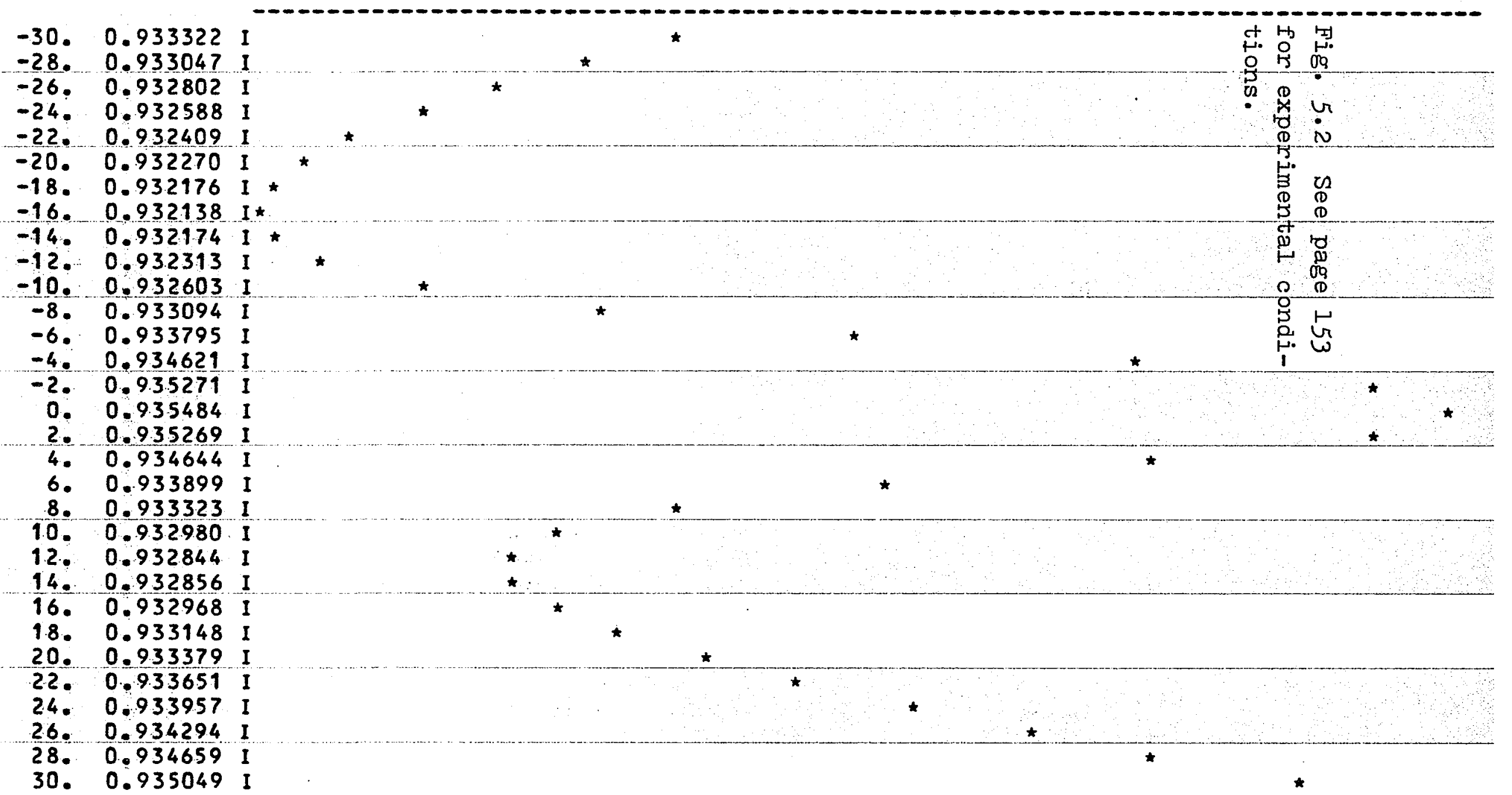

- THE NARROW STRONG \& WEAK BEAMS ARE SIMULTANEOUSLY SCANNED OVER NA F 22 LINE $(F=J+I, J=1 / 28 I=3 / 2)$ * * THE SATURATED aTOMS DETECTED BY THE WEAK BEAM aRENEARLY aT REST *

* THE PEAK FREQ IS THEREFORE THAT OF THE UNSHIFTED LINE * 
In this section we will study the line shape of light reflected from a probing beam at the interface between a solid and gaseous atoms, where atoms are physical -ly adsorbed on the solid wall. As a model system, the interface between a thin metal layer deposited on a glass surface and $\mathrm{Na}$ vapor as the gas, will be considered. In order to simplify this model interface, we first separate it into various regions by placing the hypothetical surfaces; $S_{+}$at the glass wall $(z=0), S_{0}$ at the thin metal layer surface $\left(z=d_{0}\right)$ where the molecular environment can be regarded as those of the bulk metal, and $S_{-}$at a distance $(\mathrm{z}=\mathrm{d})$ where all the field quantities vanish because of the strong resonance absorption within the transition region $V_{ \pm}$(see Fig. 5.3). Thus $V_{ \pm}$has two regions. The first region $V_{ \pm}^{(1)}$ starts at $S_{+}$and ends at $S_{0}$, and the second region $V_{ \pm}^{(2)}$ starts at $S_{0}$ and ends at $S_{-}$.

In order to further simplify our model, we assume that the thickness $\left(d_{0}\right)$ of the metal is much smaller than the wavelength of the incident light so that interference (due to the thickness of the metal) in the reflected beam can be ignored. Therefore in the simplified model we can use the extinction theorem, Eq. (3.3a), and thus the same line shape expressions are as those in section 3 of Chapter IV. However, the van der Waals' interaction between the metal and $\mathrm{Na}$ atoms may create a different environment for the gaseous atoms near the surface.

As mentioned.in section 1 , the surface potential is 
A THIN METAL IAYER(DEPOSITED ON A GIASS)-GAS INTERFACE

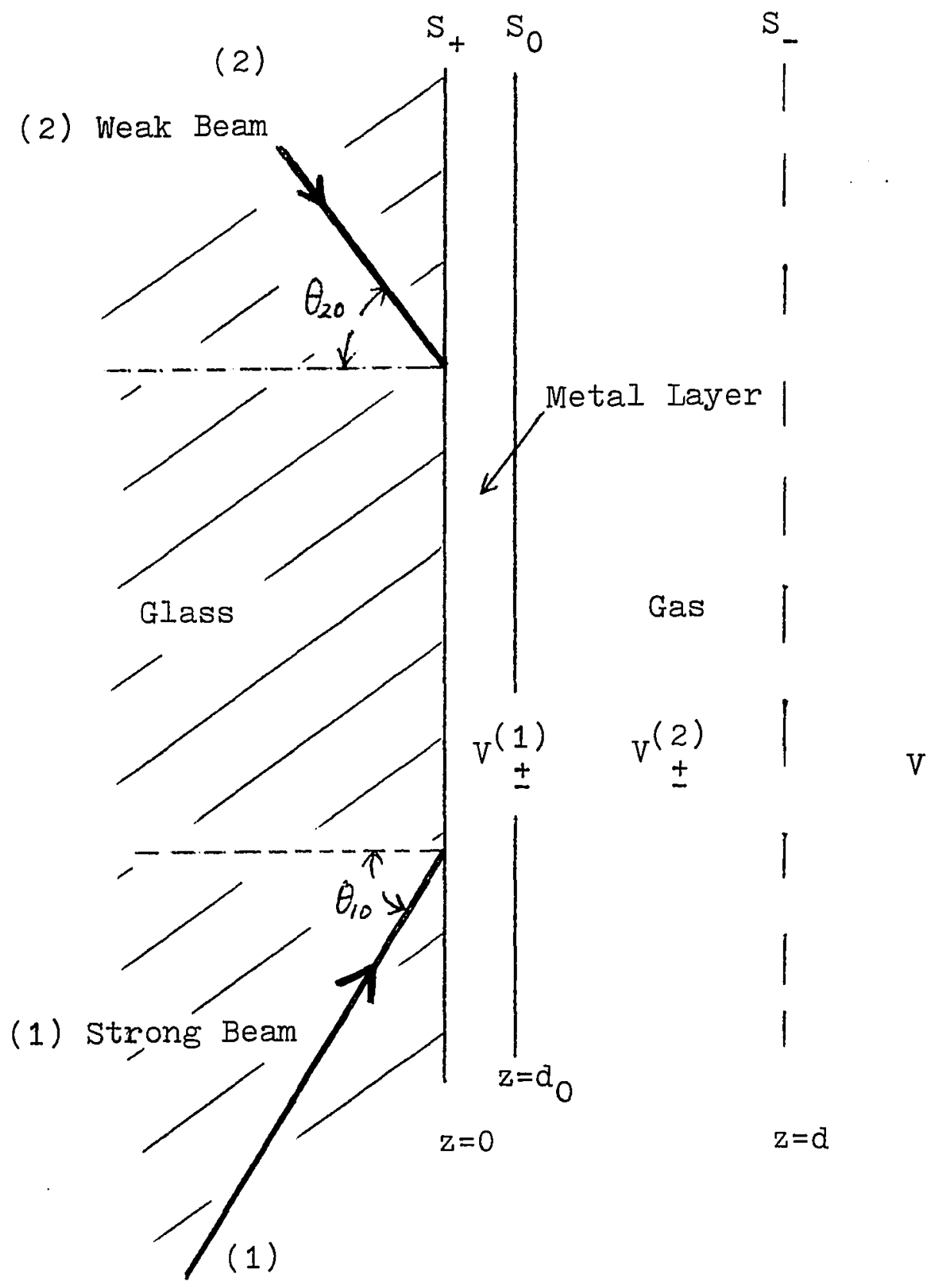

Fig $\cdot 5 \cdot 3$ 
not known. We assume here that it has the form of an inverse power of the normal distance $z$ from the surfaces with an infinite repulsive part at a small distance, $\delta$. Then the change of the resonance frequency, $\Delta V_{0 l}$, due to the potential is given by

$$
\Delta \nu_{0 \Omega}=\alpha_{P} / Z^{P}, \quad(P=2,3,4 \text {, etc. }),
$$

where $\alpha_{p}$ is a constant. In the quasi-statistical approach to line shape theory, the influence of the frequency change due to the interaction on the line shape may be investigated by first finding the macroscopic polarization vector $\vec{P}(\vec{r}, \omega)$ in $\mathrm{V}_{ \pm}^{(2)}$, and then the electric susceptibility similar to Eqs. (5.8a) and (5.8b). Because of the interactions, the reduced one particle distribution function for those atoms which are in the ground state is now given by

$$
f_{0}(z, v)=\frac{N}{V_{ \pm}^{(2)}} \exp \left\{-\frac{1}{R_{B} T} \frac{C_{p}}{z^{p}}\right\} \frac{2}{\sqrt{\pi}} \cdot \frac{1}{\Delta v} \exp \left\{-\frac{4 v^{2}}{\Delta v^{2}}\right\}\left[1-W_{0 Q}\right],
$$

with

$$
W_{c l}\left(\theta_{10}, \nu, Z, v\right)=\left[U\left(\nu-\nu^{(i)}+\frac{1}{2} \delta \nu^{(i)}\right)-U\left(\nu-\nu^{(i)}-\frac{1}{2} \delta \nu^{(i)}\right)\right] \frac{G^{2}}{2} \cdot \frac{\left(1 / 2 \pi \tau_{c}\right)^{2}}{\left(\nu-\nu_{c k}^{\prime}+\Delta \nu_{c l}\right)^{2}+\left(1 / 2 \pi \tau_{c}\right)^{2} \cdot\left(1+G_{G}^{2}\right)},
$$

where $V_{c l}^{\prime}=V_{c e}\left(1+n \frac{y_{c}}{\sin } \operatorname{in} \theta_{1}, \Delta \nu_{c l}=\alpha_{p} / z^{p}, \Delta v=2 \sqrt{2 \mathrm{k}_{B} \mathrm{~T} / \mathrm{M}}\right.$ and $C_{p}$ is constant in the interaction potential energy, $-C_{p} / z^{p}$, for the atoms in the ground state.

Using this reduced one particle distribution function, an electric susceptibility similar to Eqs. (5.8a) and $(5.8 \mathrm{~b})$ can be obtained. When the incident frequency $\nu^{(i)}$ is tuned to a shifted natural resonace frequency, $V_{o l}-\Delta V_{o l}$, where the frequency shift $\Delta V_{a l}$ due to the interaction is much 
larger than that due to the Doppler effect, the electric susceptibility is that of a region close to the surface. Its real and imaginary parts may be written as

$$
\begin{aligned}
& \eta_{e}^{r}\left(\theta_{20}, \nu\right)=-\frac{e^{2}}{2 m_{e}} \frac{f_{o l}}{(2 \pi)^{2}} \frac{1}{\nu_{b e}} \frac{N}{V_{ \pm}^{(2)}} \exp \left\{+\frac{1}{k_{B} T} \frac{C_{p}}{Z^{p}}\right\} \int_{-\infty}^{+\infty} d v \frac{2}{\sqrt{\pi}} \frac{1}{\Delta v}\left(\exp \left\{-\frac{4 v^{2}}{\Delta v^{2}}\right\}\right)\left(1-W_{o l}\left(\theta_{10}, \nu, z, v\right)\right] \\
& x \frac{\nu-\nu_{0, e}^{\prime \prime}+\Delta \nu_{o e}}{\left(\nu-\nu_{o e}^{\prime \prime}+\Delta \nu_{o e}\right)^{2}+\left(1 / 2 \pi \tau_{c}+\Gamma_{0} / 2\right)^{2}}
\end{aligned}
$$

and

$$
\begin{aligned}
\eta_{e}^{i}\left(\theta_{20}, \nu\right)= & -\frac{e^{2}}{2 m_{e}} \frac{f_{o R}}{(2 \pi)^{2}} \frac{1}{\nu_{o e}} \frac{N}{V_{ \pm}^{(2)}} \exp \left\{+\frac{1}{k_{B} T} \frac{C_{p}}{Z^{p}}\right\} \int_{-\infty}^{+\infty} d v \frac{2}{\sqrt{\pi}} \frac{1}{\Delta v}\left(\exp \left\{-\frac{4 v^{2}}{\Delta v^{2}}\right\}\right)\left[1-W_{o e}(\theta, 0, \nu, z, v)\right] \\
& \times \frac{-\left(1 / 2 \pi \tau_{c}+\Gamma_{0} / 2\right)}{\left(\nu-\nu_{o l}^{\prime \prime}+\Delta \nu_{o e}\right)^{2}+\left(1 / 2 \pi \tau_{c}+\Gamma_{0} / 2\right)^{2}}
\end{aligned}
$$

where $\nu_{D R}^{\prime \prime}=\nu_{O R}\left(1+n \frac{v}{o c} \sin \theta_{20}\right)$.

We now derive the electric susceptibility for the region away from the surface by a distance on the order of $10^{-6} \mathrm{~cm}$. In this region, i.e., between $\mathrm{z}$ and $\mathrm{z}+\Delta \mathrm{z}$, the number of atoms whose frequency shifts, $\Delta \nu_{0, e}$, are within the line width, $\delta \nu^{(i)}$, of incident strong beam is significant. Then, the real part of the electric susceptibility may be re-expressed as

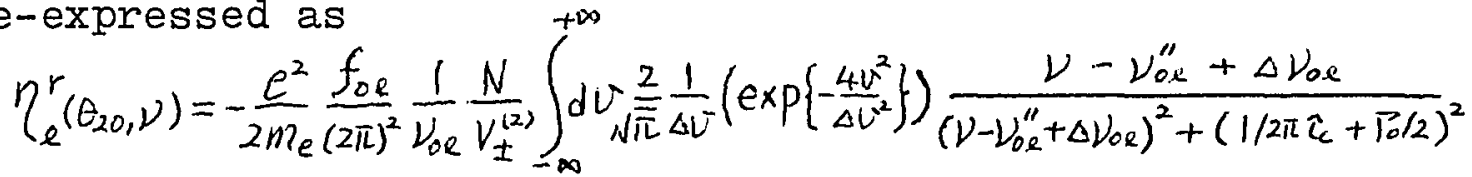

$$
\begin{aligned}
& \times\left[1-\frac{1}{C_{N}} \int^{\Delta Z} d z \exp \left\{\frac{1}{k_{B} T} \frac{C_{P}^{\prime}}{Z^{p}}\right\} W_{c k}\left(\theta_{10}, \nu, Z, v\right)\right],
\end{aligned}
$$

with the normalization constant,

$$
C_{N}=\int d z \exp \left\{\frac{1}{k_{B} T} \frac{C_{P}}{Z^{p}}\right\} \text {. }
$$


Similarly, the imaginary part may be re-expressed as

$$
\begin{aligned}
\eta_{e}^{i}\left(\theta_{20, \nu}\right)= & -\frac{e^{2}}{2 m_{e}} \frac{f_{0 R}}{(2 \pi)^{2}} \frac{1}{\nu_{o R}} \cdot \frac{N}{V_{ \pm}^{(2)}} \int_{-\infty}^{+\infty} d v \cdot \frac{2}{\sqrt{\pi}} \frac{1}{\Delta v}\left(\exp \left\{-\frac{4 v^{2}}{\Delta v^{2}}\right\}\right) \frac{-\left(1 / 2 \pi \tau_{c}+\Gamma_{0} / 2\right)}{\left(\nu-\nu_{i s}^{\prime \prime}+\Delta \nu_{o R}\right)^{2}+\left(1 / 2 \pi \tau_{c}+\Gamma_{0} / 2\right)^{2}} \\
& \times\left[1-\frac{1}{C_{N}} \int^{\Delta z} d z \exp \left\{\frac{1}{k_{B} T} \frac{C_{P}}{z^{p}}\right\} W_{o R}\left(\theta_{10}, \nu, z, v\right)\right] .
\end{aligned}
$$

In order to numerically calculate the line shape of the reflected weak beam, we will follow the procedure outlined in section 2. Some of the numerically calculated results for the case where the refracted weak beam and strong beams are counter-propagating with the incident angles $\theta_{10}=\theta_{10}=\theta_{0}$, are given in Appendix $B$.

When the strong and weak beams are simultaneously scanned, the intensity distributions of the weak beam reflected from our model interfaces are also obtained. The intensity distributions among the possible interactions are given in Fig. 5.4 . 


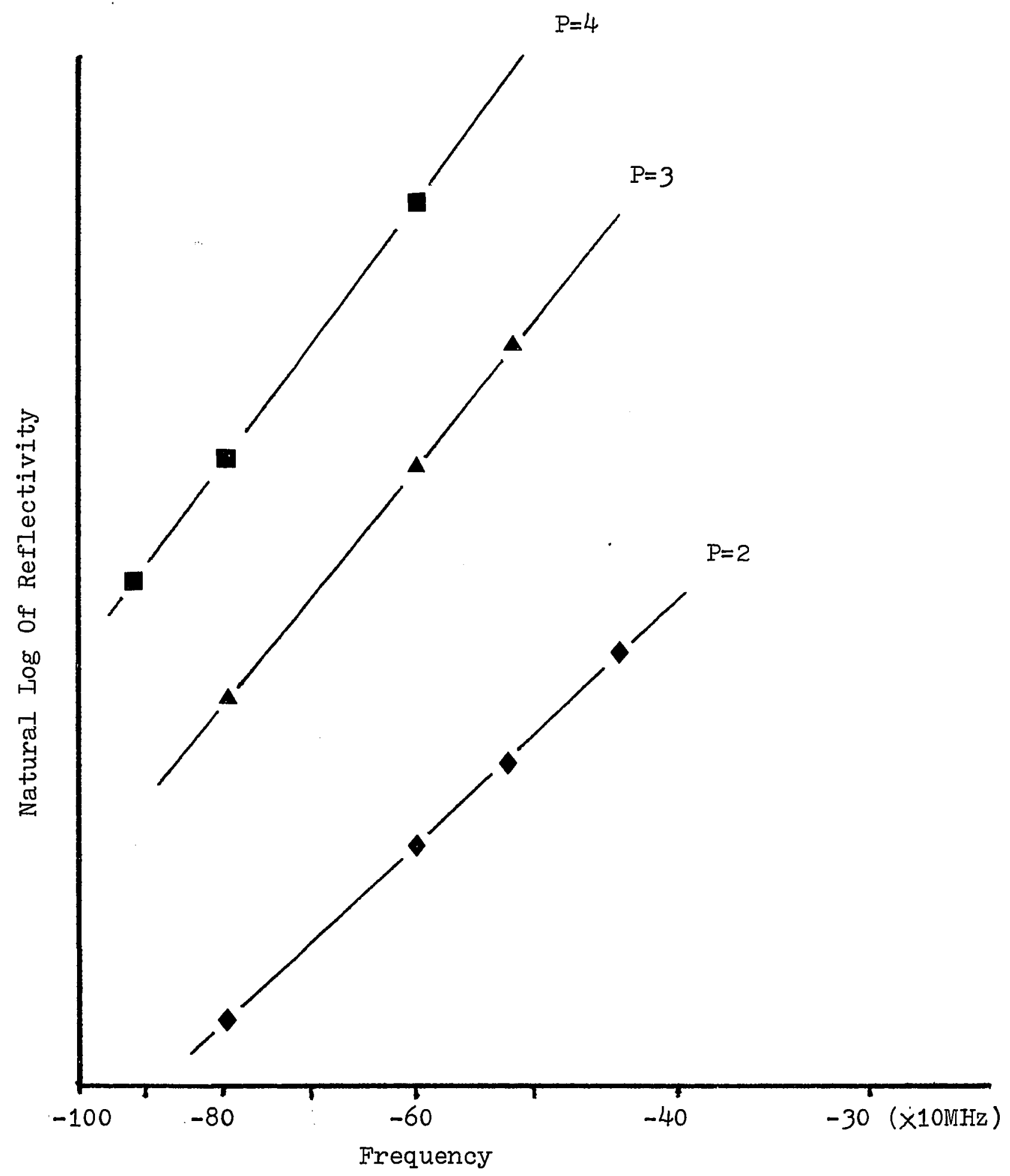

Fig. 5.4 The unshifted natural resonance line is at frequency 0. For detailed experimental conditions, see Appendix $B$. 


\section{CHAPTER VI \\ APPLICATION OF TUNABLE IAASER \\ TO GAS FLOW MEASUREMENTS \\ NEAR A WALI}

\section{INTRODUCTION}

Consider gas flow in a region near a solid wall. This region is divided into two parts: the region $V_{ \pm}$ which is within a distance of the mean free path of the gaseous atoms (or molecules) from the wall, and the remainder of the bulk gas region, $V$. When the gas flow in $V$ is laminar, the flow field may be obtained by solving the Navier-Stokes equation numerically with the usual boundary conditions. When the flow becomes turbulent, attempts to obtain the complex flow fields numerically may be made by applying the Navier-stokes equation with appropriate turbulent boundary conditions. However, these calculations will be successfull only if the rapidly changing flow fields within the boundary layer are known.

The physical flow information near the wall, such as: mean flow velocity, Reynolds' stress, turbulent kinetic energy, etc., may be obtained using a Laser Doppler Anemometer. However, it cannot be used right up to the wall because of physical limitations due to the effective 
probe volume at the beam intersection (typically $100 \mu \mathrm{m} \times$ $100 \mu \mathrm{m} \times 200 \mu \mathrm{m})$ and the diameter of seeding particles, $1 \mu \mathrm{m}$, (see the text of F. Durst, et. al., 1976). Also, when the gas becomes rarefied, the boundary conditions cannot be obtained by I.D.A.. Thus we need a new experimental technique to obtain the macroscopic flow field (appropriately averaged) in $V_{ \pm}$. Not only would we obtain the boundary conditions but also physical information for the flow field just outside $V_{ \pm}$. In fact, any observation of turbulent kinetic energy in this region $V_{ \pm}$, may help in understanding the production and dissipation process of turbulent kinetic energy in the region just outside $V_{ \pm}$, i.e., the boundary layer of the flow in $\mathrm{V}$.

Thus, the purpose of this chapter is to investigate a possible application of a tunable laser to macroscopic gas flow measurements in the region $V_{ \pm}$using the saturation spectroscopic technique which was previously studied.

\section{RESPONSE OF THE WEAK BEAM} TO GAS FLOW

We will consider the same interface between the glass and $\mathrm{Na}$ vapor as before using the saturation spectroscopic technique studied in section 2 of Chapter $v$. We assume that within the transition region $V_{ \pm}$, there is a macroscopic instantaneous flow velocity, $u(z, t)$, at a distance $z$ from the wall, and parallel to the wall. In order to define $u(z, t)$, we use the time dependent reduced one particle distribution 
function

$$
f_{0}^{0}(\vec{r}, v, t)=\left(\frac{M}{2 \pi k_{B} T}\right)^{1 / z} \frac{N}{V_{ \pm}} \exp \left\{-\frac{[v-u(z, t)]^{2}}{2 k_{B} T}\right\},
$$

where $M$ is the mass of the gaseous atom (or molecule) and $N$ is the average number in $V_{ \pm}$. We may then define $u(z, t)$ through the mass flow,

$$
\rho u(z, t)=\int_{V_{t}} d^{3} r^{\prime} \int_{-\infty}^{+\infty} d v^{\prime} \delta\left(\vec{r}^{\prime}-\vec{r}\right) M v^{\prime} f_{0}^{0}\left(\vec{r}, v^{\prime}, t\right),
$$

where the mass density is defined as

$$
\rho=\int_{V_{ \pm}} d^{3} r^{\prime} \int_{-\infty}^{+\infty} d v^{\prime} M \delta(\vec{r}-\vec{r}) f_{0}^{0}\left(\vec{r}^{\prime},\left(v^{\prime}, t\right)=\frac{N M}{V_{ \pm}} .\right.
$$

In obtaining a mean of the instantaneous $u(z, t)$, the time average over a long period of time may be changed to an ensemble average over realizations of $u(z, t)$. Denoting the ensemble average by \langle\rangle , we have

$$
\langle u(z, t)\rangle=U_{0}(z) \text { and }\langle u(z, t) u(z, t)\rangle \neq 0 \text {. }
$$

We therefore write $u(z, t)$ as

$$
u(z, t)=U_{0}(z)+\tilde{u}(z, t) \text {, }
$$

where $\tilde{u}(z, t)$ is a velocity of flow fluctuating around $U_{0}(z)$. We now study the response of the probing beam to a fluctuating velocity field $\tilde{u}(z, t)$ in terms of the numerically calculated line shape of the reflected light around the burned hole. For simplicity, let us assume the following hypothetical flow conditions within $V_{ \pm}: U_{0}(z)$ and $u(z, t)$ are independent of $\mathrm{z}$, and are so small that $\mathrm{U}_{0}<\Delta \mathrm{v}$, and $\tilde{u}(z, t)<\Delta v\left(\Delta v=2 \sqrt{2 k_{B} T / M}\right)$. The distribution of $\tilde{u}$ is assumed to be Gaussian, 


$$
f(\tilde{u})=\frac{2}{\sqrt{\pi}} \frac{1}{\Delta \tilde{u}} \exp \left\{-\frac{4 \tilde{u}^{2}}{\Delta \tilde{u}^{2}}\right\},
$$

where the Doppler shift corresponding to the effective fluctuation, $\Delta \widetilde{u}$, is larger than the line wilth of the incident beam, $\delta \nu^{(i)}$. The magnitude of $\delta \nu^{(i)}$ will then become a physical limitation on the resolution.

$$
\text { In order to numerically calculate the line shape of }
$$
the reflected probing beam, we must first obtain the real and the imaginary part of the electric susceptibility similar to those of Chapter $V$. Using the same notations as Eqs. (5.8a) and (5.8b) of Chapter V, they can be written

$$
\begin{aligned}
& \eta_{l}^{r}\left(\theta_{20, \nu}\right)=-\frac{e^{2}}{2 m m_{e}} \frac{f_{0 x}}{(2 \pi)^{2}} \frac{1}{\nu_{c l}} \cdot \frac{N}{V_{ \pm}} \int_{-\infty}^{+\infty} d \tilde{u} \frac{2}{\sqrt{\pi}} \cdot \frac{1}{\Delta \tilde{u}} \exp \left\{-\frac{4 \tilde{u}^{2}}{\Delta \tilde{u}^{2}}\right\} \int_{-\infty}^{+\infty} d v \cdot \frac{2}{\sqrt{\pi}} \frac{1}{\Delta v} \exp \left\{-\frac{4\left(v-\tilde{u}-U_{c}\right)^{2}}{\Delta U^{2}}\right\} \\
& \times\left\{1-W_{D Q}\left(\nu, U_{c}, \hat{u}, \nu, \theta_{10}\right)\right] \frac{\nu-\nu_{o e}^{\prime \prime}}{\left(\nu-\nu_{o l}^{\prime \prime}\right)^{2}+\left(1 / 2 \pi \tau_{c}+\Gamma_{0} / 2\right)^{2}}
\end{aligned}
$$

and

$$
\begin{aligned}
& \eta_{e}^{i}\left(\theta_{20}, v\right)=-\frac{e^{2}}{2 m_{e}} \cdot \frac{f_{0 R}}{(2 \pi)^{2}} \frac{1}{\nu_{i,}} \cdot \frac{N}{V_{ \pm}} \int_{-\infty}^{+\infty} d \tilde{u} \frac{2}{\sqrt{\pi}} \frac{1}{\Delta \tilde{u}} \exp \left\{-\frac{4 \hat{u}^{2}}{\Delta u^{2}}\right\} \int_{-\infty}^{+\infty} d v \cdot \frac{2}{\sqrt{\pi}} \cdot \frac{1}{\Delta v} \cdot \exp \left\{-\frac{4\left(v-\tilde{u}-U_{0}\right)^{2}}{\Delta v^{2}}\right\} \\
& \times\left[1-W_{D \ell}\left(v, U_{0}, \pi, \nu, \theta_{10}\right)\right] \frac{-\left(1 / 2 \pi \tau_{c}+P_{0} / 2\right)}{\left(\nu-\nu_{0 \ell}^{\prime \prime}\right)^{2}+\left(1 / 2 \pi \tau_{c}+P_{0} / 2\right)^{2}},
\end{aligned}
$$

where $\nu_{0.2}^{\prime \prime}=\nu_{0, e}\left(1+\frac{v-U_{0}-\tilde{u}}{c} n_{0} \sin \theta_{20}\right)$. Here $w_{0 . \ell}\left(v, U_{0}, \tilde{u}, \nu, \theta_{10}\right)$ is given by

$$
W_{O R}\left(\nu, U_{0}, \tilde{u}, \nu, \theta_{10}\right)=\left[U\left(\nu-\nu^{(i)}+\frac{1}{2} \delta \nu^{(i)}\right)-U\left(\nu-\nu^{(i)}-\frac{1}{2} \delta \nu^{(i)}\right)\right] \frac{G^{2}}{2} \frac{\left(1 / 2 \pi \tau_{c}\right)^{2}}{\left(\nu-\nu_{0 R}^{\prime}\right)^{2}+\left(1 / 2 \pi \tau_{c}\right)^{2} \cdot\left(1+G^{2}\right)},
$$

where we recall that $\nu_{0 \ell}^{\prime}=\nu_{0 \ell}\left(1+\frac{v-U_{0}-\tilde{u}_{u}}{c} n_{0} \sin \theta_{10}\right)$, and $G^{2}=e^{2} f_{o u}|\vec{E}|^{2} \hat{L}_{c} \tau /\left(4 m_{e} V_{o . e} h\right.$ ) (see section 2 in chapter $V$ ). We then follow the procedure outlined in sections 2 and 3 of Chapter $V$ to obtain the line shapes of the reflected weak 
beam. Some of these results are given in Appendix $C$. 


\section{REFERENCES}

Balescu, R. 1975. Equilibrium and Nonequilibrium Statistical Mechanics, John Wiley, New York, Ch. 3.

Bennett, W. R. Jr. 1962. Phys. Rev. 126, 580.

Born, M. and Wolf, E. 1975a. Principles of Optics, 5th ed., Pergamon Press, New York, Sec. 2.4.2.

Born, M. and Wolf, E. 1975b. Ibid. Sec. 1.1.3.

Chen, S. Y. and Takeo, M. 1957. Rev. Mod. Phys. 29, 20.

Cojan, J. I. 1954. Annales de Physique, 9, 385.

Durst, F. J., Melling, A. and Whitelaw, J. H. 1976. Principles and Practice of Laser-Doppler Anemometry, Academic Press, New York.

Hänsch, T. W., Schawlow, A. L. and Series, W. 1979. Scientific American, Mar., pp. 94-110.

Jackson, J. D. 1975. Classical Electrodynamics, 2nd. ed. John Wiley, New York, Sec. 6.7.

Karplus, R. and Schwinger, J. 1948. Phy. Rev. 73, 269.

Ialor, E. 1968. J. Dpt. Soc. Am. 58, 1235.

Ialor, E. and Wolf, E. 1972. J. Opt. Soc. Am. 62, 1165.

Landau, I. D. and Lifshitz, E. M. 1960. Electrodynamics of Continuous Media, Pergamon Press, New York, Sec. 66.

Lange, W., Iuther, J. and Steudel, A. 1974. Advances in Atomic and Molecular Physics, Bates, D. R. and Bederson, (eds.), Academic Press, New York, 10, pp. 173 -221 .

Launder, B. E., Hanjalić, K., Dwyer, H. A. and Durst, F. J. 1978 and 1979. Summer Lecture Notes on Measurement and Prediction of Complex Turbulent Flows, Univ. of California, Davis.

Morse, P. M. and Feshbach, H. 1953. Method of Theoretical Physics Part II, McGraw Hill, p. 1779. 
Muzar, P. and deGeode, J. 1972. Physica, 58, 585.

Nir, S. 1976. Progress in Surface Science, 8, 1.

Pattanayak, D. N. and Wolf, E. 1972. Opt. Commun., 6, 217.

Schrieffer, J. R., Soven, P., Estrup, P. J., Eastman, D. E., Nathan, M. J., Park, R. I., Plummer, E. W., Gadzuk, J. W. and Penn, D. R. 1975. Physics Today, April, pp. 24-72.

Sein, J. J. 1970. Opt. Commun., 2, 170.

Shewell, J. R. and Wolf, E. 1968. J. Opt. Soc. Am., 58, 1596.

Shih, A. and Parsegian, V. A. 1975. Phy. Rev. A., 12, 835.

Straton, J. A. 194I. Electromagnetic Theory, McGraw Hill, New York, Sec. 9.29.

Takaishi, T. 1975. Progress in Surface Science, 6, 45.

Takeda, F. and Takeda, R. 1979. "The Calibration Eqs. of Propeller Current Meters for Turbulent Flows", 79WA/FM-2, ASME, to be published in ASME J. FIuid Engng.

Takeda, F. and Takeo, M. 1980. "Theory of Surface Effect on Line Shapes", submitted to J. Quant. Spectro. Radiat. Transfer.

Towens, C. H. and Schawlow, A. I. 1955. Microwave Spectroscopy, McGraw Hill, New York, Sec. 13.15 .

Weyl, H. 1919. Ann. Phys. Ipz., 60, 481.

Woerdman, J. P. and Schuurmans, M. F. H. 1974. Private Commun.

Wolf, E. 1976. Symposia Mathematica, Vol. XVIII (Istituto Nazionale di Alta Matematica), Academic Press, New York, pp. 333-352.

Wood, R. W. 1934. Physical Optics, 3rd. ed., Macmillan, New York. 


\section{APPENDIX A}

Honeywell 66-20 computer at P.S.U. was used for the numerical calculations. The following programs were written in terms of Fortran. The control command words for the TSS terminal and batch interface were preceded by $\$$ signs in the listed programs. 
\$ \$ JOUT, MONI, T

\section{$\$$}

INENT

RFHYAO01, SELEC REF

OFTION FORTRAN

FOFTFIAN

C

c

CALCULATION

LIGHT FROM A GLASS- GAS INTERFACE

SIMPSON'S METHON IS USED IN NUMERICAL INTEGRATIONS

TO DETAIN THE WALL EFFECT, DFTICAL FARAMETEF OF EULK GAS

IS USEI AS AN INITIALIZATION IN THE ITERATIONS

C

C

C

WHEN ISINGLE=3 (SEE RELOW), THE CANGES OF THE UPPER LIMITS

FOR $+Z$ COMFONENT OF THE THERMAL VELOCITY FOR EACH LINE

C. ARE DRTAIEI EY USING THE EULK OFTICAL FAFAMETER IIUE

C TO THE TWO RESONANCE LINES (EXCEFT WHEN "ANGINC" IS LAFGER THAN

C A CRITICAL ANGLE)

C

THIS METHON SAUES A CONSIIIERARLE AMOUNT OF $C . F$. TIME TO OETAIN THE SAME RESULTS

\section{*CONTFOL INTEGERS*}

FOF IPFOGM=0, INCIUENT LIGHT WILL EE WHITE

FOF IPFOGM=1, INCIIENT LIGHT WILL BE A NAFFOW GAUSSIAN BEAM

ANII SCANNEI OUER NA I2 LINES

FOF ISINGLE =1, ONLY THE LINE (F=2) WILL BE EXAMINEI

WHERE $F=J+I, \quad(J=1 / 2 \& I=3 / 2)$

FOF ISINGLE=2, ONLY THE LINE $(F=1$ ) WILL EE EXAMINEI

WHERE $J=-1 / 2 \& I=3 / 2$

FOF ISINGLE $=3$, ROTH LINES WILL BE EXAMTNEI

IFOLARI $=-1$; FARALLEL FOLARIZATION

TPOLART $=0$; FLANE FOLARIZEI LIGHT AT 45 REGFEE

IFOLARI $=+1$; FAFFENITCULAR FOLART ZATION

\section{*CONSTANTS*}

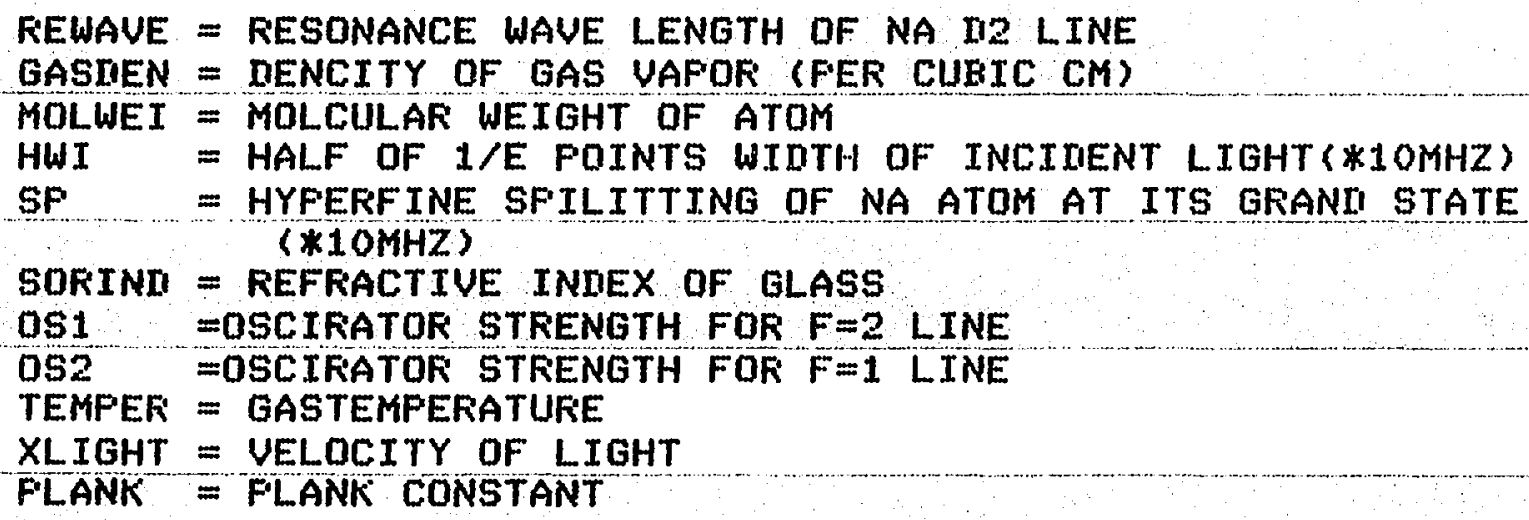


- C ELMASS = ELECTORON MASS

c $\quad$ GASCON $=$ GAS CONSTANT

XLOSHM $=$ LOSHMINT'S NUMEEF

C AANGLE = ANGLE OF INCIIENCE (DEGREE)

C ANGINC = ANGLE OF INCIIECE (FAAIIAN)

C EFDOF = 1/E FOINTS WINTH OF DOPPLEF BFOAIENEU NA II2 LINE

C $P A I=P A I$

IIIMENSION $Z(58)$, REINAT (5B), REFRET (58), REFCMT (58), REFT (58),

\& IIEFTH (58), UFFEER1 (10), UFFER2 (10), REINI (58), IIIERE (58), $\operatorname{IIECM}(58)$,

\& REF (58), ANGRET (58), ANGREN (58), MEFTHN (58), RET (5)

GASREN $=1.05 E+15$

$X X E=A L O G(2$.

$H W I=20 . /$ SRRT (XXE)

TEMPER $=619$.

FOLARI $=+1$

ISINGLE $=3$

IF $R O G M=1$

REWAUE $=5,890 \mathrm{E}-5$

$X M O L W E=22+9898$

$\mathrm{SF} \quad=177.2$

SORINII $=1.63$

DSI $=0.4166301$

IF (ISINGLE,ER . 2)0S1=0.

QS2 $=0.2500363$

IF (ISINGLE+ER, 1)052=0 .

$X L I G H T=2,99793 E+10$

FLANK $=1.05459 E-27$

ELMASS $=9,10953 E-28$

GASCON $=8 \cdot 31441 E+7$

FAI $=3.14159$

$X$ LOSHM $=2,68675 E+19$

NOF $=2+* G A S C O N * T E M F E R / X M O L$ WE

EFCDOF $=2$ * *SRRT ( DOF) /REWAUE

$X M H Z=10+E+6$

$X E M 7=1 \cdot E-7$

C HALF OF HALF -UALUE WIUTH OF RESOMACE BROADENEI LINES

C HWN1 IS FOR $F=2$ \& HWN2 IS FOR $F=1$

HWN $1=0.8142 * \times$ EMT $*$ GASNEN $/ X M H Z / 2$.

HWN2 $=0.5543 * X E M 7 * G A S D E N / X M H Z . / 2$.

EF NOF $=$ EFCIIOF/XMHZ

c

c CONST=CONSTANT APPERING IN SUSCEPTIBILITY

C

CONST $1=$ FLANK $*$ GASNEN*REWAUE

CONST $2=137.036 * E L$ MASS $* S Q R$ T (FAI) $* 4$, *EFCIOF $* F A I * * 2$

CONST $=$ CONST1 1 CONST2

IPOLA $=$ IFIX (FOLARI)

898 FORMAT (IH, 3X, FOLARIZATION IS PARALLEL?)

897 FORMAT (1H, 3X, "FOLARIZATION IS PERFENIICURAR")

896 FORMAT (1H , 3X, "PLANE FOLARIZEN LIGHT AT 45 IEEGREE")

c

c VAPOR=VAFOR FRESSURE IN UNIT OF MMHG

c

UAFOR $=760, *$ TEMFER $* G A S D E N / X L O S H M / 273.2$ 
900 FOKMAT (1H, 3X, "RESONANCE WAVE LENGTH OF NA II LINE" "

$2 \quad 23 X,{ }^{*}=" E 10,3,2 X, *(C M) \cdots / 1 H, 3 X$

\& "SFILITTING OF HYFERFINE IOURLET AT GRANR STATE","

\& $23 X, "=", F 6,1,4 X, "(* 10 \mathrm{MHZ}) ", / 1 \mathrm{H}, 3 X$, "IENSITY OF" ,

\& VAFOR", 3X,"=",E9,3,1X" "(FER CUBIC CM)", $1 H$,

\& $3 X$, "VAFOR PRESSURE", $\bar{X}, "=", F 7,3,3 \bar{X},(M M H G), / 1 H$,

\& $\quad 3 X$, TEMFERATURE OF GAS*,1X,"=",F5,0,11H,3X,

8 'HALF OF $1 / E$ FOINTS' WIUTH OF INCINENT LIGHT" $/ 23 X$,

$\& \quad=", F 6,1,5 X, "(* 1 O M H Z) ", / 1 H, 3 X, " 1 / E$ FOINTS" [IOFFLER",

\& "WINTH", $/ 23 X, "=", F G, 1,4 X, "(* 1 O M H Z) "$,

$8 \quad 11 H, 3 X$, "HALF OF HALF WIITH OF" ",

\& FRESSURE HROAIIENEI LINE $1 \%$

\& $23 X, "=", F 5,1,5 X, "(* 1 O M H Z) ", 11 H, 3 X, " H A L F$ OF HALF WIIITH",

* OF FRESSURE BROAIENER LINE $2 ", / 23 X, "=", F 5,1,5 X, "(* 1$ OMHZ)*,/)

$X K E F=1+E-6$

$Z E K O=0$.

$N A=6$

$F N A=F L O A T(N A)$

EFHWI $=1$ + $6 * H W I$

$H A=2$ * *EFHWI/FNA

$\angle L A=15$

DO 300 LLAN=10,55, LLA

LANGLE =LLAN-10

AANGLE=FLOAT (LANGLE)

ANGINC=AANGLE*FAI $/ 180$.

SORSIN=SORINIISSIN (ANG INC)

MOFS $=$ EF IOF $*$ SORSIN

TLOW $=-0+8 * E F D O F$

THIG $=0.8 *$ EFIIOF

FNTT $=0$ + 8*EFROF $/ 4$.

IF (IFROGM + EQ , 0)FNTT $=0$, 8*EFnOF $/ 2$.

NTT $=$ IFIX (FNTT)

FNT $=2$, *FLOAT (NTT)

$N T=I F I X(F N T)$

$H T=1$. $6 * E F M O F / F N T$

IF (LANGLE,EQ,O)GO TO 4

$F N D 1=n O P S * 0.8 / 4$.

NDI =IFIX (FNn1)

FND $=F L O A T(N H 1) * 2$.

$N D=I F I X$ (FND)

$H D=1$. G*MOFS/FND

C XUFFAC IS A TRANCATION FACTOR FOR THE UFFER LIMIT IN THE

C. AUERAGE QUER $Z$ COMFONENT OF THERMAL VELOCITY

$4 \quad$ XUPFAC $=1,2$

WFITE $(6,69)$

69 FORMAT $(1 H 1, / / / 4 X, "<$ UFPER LIMIT FOR UELOCITY AUERAGE $\% ")$

IFREQ $=270$

$\mathrm{FSC}=30$.

RAT IO=FLOAT (IFRER )/FSC+0.5

RATTOZ=3, *RATTO

IC $=$ IFIX (FATIOZ)

no $200 \quad \mathrm{IZ}=1, \mathrm{IC}$

IIZ $=I Z-1$

C Z(IZ) IS THE FREQ(OR CENTFAL FREQS OF INCIIENT LIGHT W.R.T 
C THE CENTRAL FFEQ OF THE LINE OF $F=2$ $Z(I Z)=-F L D A T(I F R E Q)+F L O A T(I I Z)$ KFSC

$S L O W=Z(I Z)-E F H W I$

C SIMFSON'SMETHON STARTS TO TAKE A CONUOLUTION INTEGRAL W.F.T

C A NAFFOW SFECTFAL LINE WIITH OF INCIIENT LIGHT

$S B=0$.

$S R G=0$.

SSUSR $=0$.

SSUSC $=0$.

$S B T=0$.

SUSRT $=0$.

SUSCT $=0$.

IF (IPFOQM +ER . O) NA=O

I0 $600 \quad \mathrm{JR}=1, \mathrm{NA}+1,2$

IF (IFFOGM +EQ + O) GO TO 308

$J \mathrm{ABB}=\sqrt{\mathrm{N} B-1}$

HE $=$ HA $* F L O A T$ ( JEE)

$X B=5 L O W+H B$

$S M B=0$.

$S M B G=0$.

SMSUSF $=0$.

SMSUSC $=0$.

SMBT $=0$.

SMSURT $=0$.

SMSUCT $=0$,

LL.BR $=2$

308 IF (IFROGM +ER + O ILLER=1

IIO 650 LLE $=1$, LLEE

IF(IFKOGM,EQ + 0)GO TO 301

$L E=L L B-1$

$F L=F L D A T(L B)$

$X A=X B+H A * F L$

CALL GAUSIA(AIGAUS, Z(IZ), XA, HWT, FAIS)

GAUS=AIGAUS

301 IF (IFFOGM +EQ + O) XA=Z(IZ.)

ILINE $=1$

CALL SUSCEP (RESUM, CMSUM,NT, XA,HT, TLOW, SP, CONST,

\& OS1, OS2,EF NOF , HWN1, HWN2, IL INE)

FESUM21=RESUM

CMSUM $21=$ CMSUM

ILINE $=2$

CALL SUSCEP (FESUM, CMSUM, NT, XA,HT, TLOW, SF, CONST,

\& 051, 052, EF IOF , HWN1, HWN2, ILINE)

RESUM22 = FESUM

CMSUM22 =CMSUM

RESUM2=FESUM21+KESUM22

CMSUM2=CMSUM21+CMSUM22

IF (ISINGLE,EQ . 3) 90 TO 400

CALL. REFIND ( FE INIIX, REFFEX, FEFCMX, ALFAX, EETAX, FESUM22,

\& CMSUM22, SORINI, ANGINC, PAI)

REINI22 $=$ REINIX

REFFE22=REFREX

REFCM22=REFCMX

ALFA22 $=A L F A X$ 
HETA22 $=$ BETAX

XANGLE22=5OFSIN/FEINM22

ANGFEF 22=ARS IN (XANGLE22)

ANGRE22=180. *ANGREF22/FAI

CALL REFINI (FEINIX, FEFREX, FEFCMX, ALFAX, BETAX, FESUM21,

\& CMSUM21, SOFINI, ANGINC, FAI)

REIND21 =REINIX

REFRE21 =FEFREX

FEFCM21 =FEFCHX

ALFA21 =ALFAX

BETA21 = BETAX

XANGLE21 =5ORSIN/REIND21

ANGREF21=ARSIN (XANGLE21)

ANGLE21 = 180. *ANGREF21/FAI

400 CALL REFINII REINIX, REFFEX, FEFCMX, ALFAX, BETAX, FESUM2,

8 (CMSUM2, SORINR, ANG INC , FAT)

FEINIZ=FIEINIX

REFRE2 =REFREX

FEFCM2=FIEFCMX

ALFA2=ALFAX

BETA2=BETAX

XANGLE $=$ SORSIN/FEINLI

ANGREF = ARSIN (XANGLE)

ANGRE2 $=180$. *ANGREF / FAI

CALL REFLEC (FEFLFX, ALFA2, EETA2, ANGINC, ANGFEF, FOLARI,

2. SOFINIIS

REF2 $=$ KEFLFX

IF(ISINGLE,ER +3)GO TO 401

IF (LANGLE.ER . 0) CN1=CONST/ALFA21

IF (LANGLE, EQ . O) CN2=CONST/ALFA22

IF (LANGLE, GT . O) CN1=CONST/ALFA21/IIOFS

IF (LANGLE + GT + O) CN2=CONST/ALFA22/LOFS

CKI TAN $=80$.

IF (ANGFE2, GT , CRITAN) CN=CONST/SORSIN

IF (ANGFE2, GT,CFITAN) GO TO 11

IF (BETA21 + LE , XREF) TUP $1=$ THTG

IF (BETA22, LE + XFEF) TUF2=THIG

IF (BETA21, GT . XFEF) TUF $1=X U F F A C * A L F A 21 /$ BETA21

IF (EETA22, GT . XFEF) TUF $2=H W N 2 * X U F F A C * A L F A 22 / H W N 1 /$ HETA22

TLOW1 $=$ TLOW*ALFA21

TLOW2 =TLOW*ALFA22

60 To 402

401 IF (LANGLE +EQ + O) CN1 =CONST/ALFA2

IF (LANGLE,EQ . O) CN2 =CONST/ALFA2

IF (LANGLE +GT.0) CN $1=$ CONST/ALFA2/IIOFS

IF (LANGLE, GT . O) CN2=CONST/ALFA2/LIOFS

CRITAN $=80$.

IF (ANGRE2, GT , CR I TAN) CN $=$ CONST/SORSIN

IF (ANGRE2, GT + CFITAN) GO TO 11

IF (AETA2 + LE, XFEF) TUF $1=$ THIG

IF (BETA2 + LE . XFEF) TUF $2=$ THIG

IF (BETA2, GT +XFEF) TUP1 =XUFFAC *ALFA2/HETA2

IF (BETA2, GT + XREF) TUF2=HWN2*XUPFAC*ALFA2/HWN1/EETA2

TLOW1 $=$ TLOW*ALFA2 
TLOW2=TLOW1

402 TWIIT 1=TUF1-TLOW1

TWIIT $2=$ TUF $2-$ TLOW2

$\mathrm{TH} 1=\mathrm{TWIIIT} 1 / 4$.

IF (LANGLE, GT . 0) TH1=TWIAT $1 / 8$.

NNTH1 $=I F I X(T H 1)$

FNTH1 =2, *FLOAT (NNTHI)

NTH1=IFIX (FNTH1)

THX1 =TWTIT1/FNTH1

THZ=TWIIT $2 / T H X 1 / 2$.

NNTH2 =IFIX (TH2)

FNTH2 $=2$, *FLOAT (NNTH2)

NTHZ $=$ IF IX(FNTH2)

IF (ISINGLE +ER . 3) ALFA21 =ALFA2

IF (ISINGLE +EQ + 3) ALFA2 $2=A L F A 2$

IF ( ISINGLE +ER + 3) EETA21 =EETA2

IF ( ISINGLE, EQ + 3) EETA22=RETA2

IF (ISINGLE +EQ . 3) ANGRE 21=ANGRE2

IF ( ISINGLE.EQ + 3) ANGRE22=ANGRE2

IF (TUF1-THIG) $10,10,20$

10 ILINE $=1$

CALL SUSCER ( FESUM, CMSUM, NTH1, NTH2, XA, THX1, TLOW1, SF, CN1,

\& 051, 0S2, EFIIOF, HWN1, HWN2, NOFS, ALFA21, BETA21,

\& I. ANGLE, ANGINC, ANGRE21, NI, HD, ILINE)

GO TO 21

20 ILINE $=1$

CALL SUSCER (RESUM, CMSUM, NT, NT, XA,HT, TLOW, SF, CNI,

\& 0S1, 0S2, EF IOF, HWN1, HWN2, MOPS, ALFA21, BETA21,

\& LANGLE, ANGINC, ANGFE21, NI, HD, ILINE)

21 SUSRE 11=RESUM

SUSCM11 $=$ CMSUM

IF (TUP2-THIG) $30,30,40$

$30 \quad$ ILINE $=2$

CALL SUSCER (RESUM , CMSUM, NTH1, NTH2, XA, THX1, TLQW2, SF, CN2,

\& 051, 052, EF IOF, HWN1, HWN2, DOFS, ALFA22, EETA22,

\& LANGLE, ANGINC, ANGRE22, NI, HD, ILINE)

GO TO 41

$40 \quad$ ILINE $=2$

CALL SUSCER (RESUM, CMSUM,NT, NT, XA, HT, TLOW, SF, CN2,

Q 051, OS2, EF IIOF, HWN1, HWN2, NOFS, ALFA22, BETA22,

\& LANGLE , ANGINC, ANGRE22, NI, HL, ILINE)

41

SUSRE $12=$ RESUM

SUSCM12=CMSUM

CALL REFINI (FEINIX, FEFREX, REFCMX, ALFAX, EETAX, SUSRE 11 , SUSCM11,

\& SORINI, ANGINC, PAI)

FEINII $1=$ FE INIXX

IIECM 1 1 = KEFCMX

DIERE $11=$ FEFREX

ALFAN11 =ALFAX

BETAN1 1 = BETAX

CALL FEFIND (FEINIX, REFREX, FEFCMX, ALFAX, EETAX, SUSRE12, SUSCM12,

$\$$ SORINII, ANGINC, F'AI)

FEIND1 2=REINRX

IIIECM12=REFCMX 
DIERE 1 2=REFREX

ALFAN12 =ALFAX

BETAN12=BETAX

IF (ISINGLE,EQ, 3) G0 TO 403

IF (BETAN11 1 LT,$X F E F)$ TUF $1=$ TUF 1

IF (BETAN 12,LT . XREF ) TUF $2=$ TUF2

IF (BETAN1 1 + GE + XFEF) TUF $1=$ XUFFAC $* A L F A N 11 /$ BETAN 1

IF (BETAN12, GE, XFEF) TUF2=HWN2 *XUFFAC *ALFAN12/EETAN12/HWN1

IF (TUF1 + GE , THIG + ANI + TUF 2 , GE + THIG) GO TO 185

IF (TUF1 , GE . THIG + AND, TUF2, LT , THIG) GD TO 71

ILINE $=1$

C THIS ITERATION STARTS FOR ISINGLE $=1$

Do $199^{\circ} \quad N=1,10$

UFFEF $1(N)=$ XUPFAC*ALFAN11/BETAN11

$I F(N+E R+1)$ GO TO 190

$\operatorname{IIF} 1=U \mathrm{UFFF} 1(N-1)-U F \cdot \mathrm{EF}_{1}(N)$

$A \cap I F 1=A B S$ ( IIFI)

XADIF $1=A N I F 1-1$.

IF $(N+E R+4) G 0$ TO 190

IF (XARIF1.LT,ZERO) GO TO 71

IF $(N+G E+3)$ UFFER $1(N)=0.5 *(U F F E R 1(N-1)+U F F E R 1(N))$

$N N=N-1$

WRITE $(6,191$ )NN, UFFER1 (NN), N,UFFER1 (N), IIF $1, Z(I Z)$

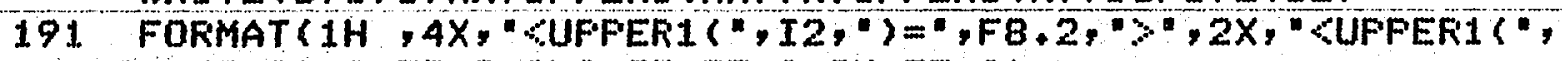

\& $I 2, " I=", F 8,2, " O " 2 X, F 5,0,2 X, F 5,0)$

$190 \quad$ XLOWEF $1=-$ THIG*ALFAN11

WIITH $=$ UFFER 1 (N) $-X$ LOWEFI

YNT $=$ WI ITTH $/ 2$.

IF (LANGLE.GT . O) YNT $=W I I T H / 4$.

NNT $=$ IFIX $(Y N T)$

YNTT $=2+* F L O A T(N N T)$

NNTT $=$ IFIX $(Y N T T)$

$\mathrm{YHT}=$ WIITH/YNTT

IF (LANGLE, EQ , O) CN1=CONST/ALFAN11

TF(LANGLE, GT +O) CN1=CONST/ALFAN11/MOFS

CALL SUSCER (RESUM, CMSUM, NNTT, NT2, XA, YHT, XLOWER1, SP, CN1,

\& 0S1,052, EFDOF, HWN1, HWN2, HOFS, ALFAN11, BETAN1 1, LANGLE, ANG INC,

\& ANGRE21,ND,HD, ILINE)

RESUM11 =RESUM

CMSUM11 $=$ CNSUM

CALL FEFINI (FEINMX, REFREX, FEFCMX, ALFAX, BETAX, RESUM 11 , CMSUM11,

$\&$ SORIND, ANGINC, FAI)

REINDI1 1 REINLX

REFRE $11=$ REFREX

REFCMI $1=$ REFCMX

ALFAN11 $1=$ ALFAX

BETAN11 = BETAX

199 CONTINUE

C THIS ITERATION STAFTS FOF ISINGLE $=2$

C

71 ILINE $=2$

IF (TUF2, GE, THIG)GO TO 187

no $75 N=1,10$ 
UFFEF $2(N)=$ HWN2*XUFFAC*ALFAN12/BETAN12/HWN1

IF (N.EQ.1) 60 TO 76

IIIF2=UFFER2(N-1)-UFFER2(N)

AIIF2=AES ( IIF2)

XAIIIF2=ADIF2-1.

IF $(N+E Q, 4) G 0$ TO 76

IF (XANIF2+LT +ZERO) GO TO 187

IF $(N, G E, 3) \operatorname{UPFEF} 2(N)=0.5 *(U F F E R 2(N-1)+U F F E R 2(N))$

$N N=N-1$

WFIT TE $(6,77) N N, U F F E F 2$ (NN), $N, U F F E F 2(N)$, IIIF2, XA

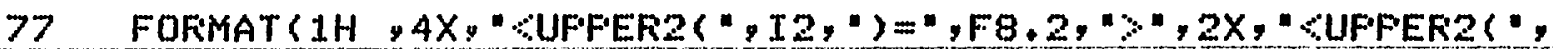

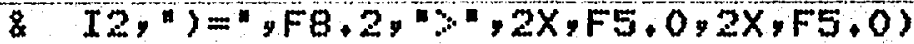

76 XLOWER2=-THIG*ALFAN12

WIDTH2=UFFER2(N)-XLOWEF2

YNTT2 $=$ WIITHIII

TF (LANGLE, GT, O)YNTT2=WIITH2/4,

NNT2=IFIX (YNTT2)

FNT2=2, *FLOAT (NNT2)

NT2 $=$ IFIX (FNT2)

YHT $=$ WIITH2/FNT2

IF (LANGLE.EQ.0) CN2 $=$ CONST/ALFAN12

IF (LANGLE.GT . O) CN2=CONST/ALFAN12/IOFS

CALL SUSCER \& FESUM, CMSUM, NNTT , NT2, XA, YHT, XLOWER2, SF, CN2,

\& 0S1, 052, EFIOF, HWN1, HWN2, IIOFS, ALFAN12, BETAN12, LANGLE, ANG INC,

\& ANGKE22, NII,HI, ILINE)

FESUM12=RESUM

CMSUM12=CMSUM

CALL FEFINI \& FETNIX, REFREX, REFCMX, ALFAX, HETAX, RESUM12, CMSUM12,

\& SOFINI, ANGINC, FAI)

REINUI2 $=$ FEINIX

REFRE 12=FEFFEX

FEFCMII $=$ FEFCMX

ALFAN12=ALFAX

BETAN12=EETAX

75 CONTINUE

WFITE $(G, 192$ ) (UFFEFI (N), UFFEF2 $(N), N=1,10)$

192 FORMAT $(4 X, " \angle U F F E F I=" F 10,2, " \%, 2 X, " \triangle U F F E R 2=", F 10,2, " \%, " 1)$

GO TO 187

11 ALFAN1 =ALFA2

C THIS ITEFATION STAFTS WHEN THE INCIIIENT ANGLE IS

C LAFGER THAN A CFITICAL ANGLE

TIO $13 \mathrm{NCO}=1$, 5

$\operatorname{BET}(N C O)=\mathrm{BETA}$

IF (NCO,ER.1)GO TO 14

IIEET $=1$ - $\mathrm{BET}(\mathrm{NCO}) / \mathrm{BET}(\mathrm{NCO}-1)$

AIUEET $=$ AES (IIEET)

FEFEET $=.01$

IF (AIIBET , LE, FEFHET) GO TO 15

14 CALL SUSCEF (FESUM, CMSUM,NT , NT , XA,HT, TLOW, SF, CN,

\& 051, 052, EFLIOF, HWN1, HWN2, IIOFS, ALFAN1, BET (NCO),

$\&$ LANGI E, ANGINC, ANGRE2, NI, HI, ILINE)

FESUMI $=$ FESUM

CMSUMI =CMSUM

CALL REFIND (FEINIX, FEFFEX, FEFCMX, ALFAX, EETAX, RESUM1, 
\& CMSUM1, SOFINI, ANGINC, FAI)

ALFAN1 = ALFAX

BETAN $1=$ BETAX

FEINI1 =FEINIX

FEFFE 1 = FEFFEX

REFCM $1=$ FEFCMX

13 CONTINUE

15 RETAN1 $=$ BET (NCO)

GO TO 186

185 IF (LANGLE+ER , 0) CN1 =CONST/ALFAN11

IF (LANGLE +EQ . O)CN2=CONST/ALFAN12

IF (LANGLE, GT * O) CN1 =CONST/ALFAN11/ ROFG

IF (LANGLE, GT , O) CN2=CONST/ALFAN12/IIOFS

ILINE $=1$

CALL SUSCER ( FESUM, CMSUM,NT, NT, XA,HT, TLOW, SF, CNI,

\& 0S1, OS2, EFIOF, HWN1, HWN2, IOOFS, ALFAN11, EETAN11,

\& LANGLE , ANGINC, ANGFE21 , NI , HI, ILINE)

FESUM $11=$ RESUM

CMSUM1 1 =CMSUM

ILINE $=2$

CALL SUSCER (FESUM, CMSUM, NT, NT, XA,HT, TLOW, SF, CN2,

\& 0S1, DS2, EFLIOF, HWN1, HWN2, IOFS, ALFAN12, BETAN12,

\& LANGLE, ANGINC, ANGRE22, NI, HI, ILINE)

RESUM12=FESUM

CMSUM12=CMSUM

60 TO 187

403 CNN $1=$ ALFA2/ALFAN11

CNN2 $=A L F A 2 / A L F A N 12$

FESUM1 =CNN1*SUSFE $11+$ CNN2*SUSFE 12

CMSUM1 $=$ CNN1*SUSCM11+CNN2*SUSCM12

60 TO 404

187 FESUM $1=$ FESUM $11+$ FESUM 12

CMSUM $1=$ CMSUM11+CMSUM 12

404 CALL FEFINII (FEINIX, REFFEX, REFCMX, ALFAX, BETAX, RESUM1, CMSUM1,

\& SOFINI, ANGINC, F'AI)

FEINII $1=$ FEINIXX

IIIECMI $=$ FEFCMX

IIEFE $1=$ REFREX

AL.FAN $1=A L F A X$

BETAN1 = EETAX

$186 \quad$ XANGLE $=$ SOFSIN/FEINII

ANGFEF = ARS IN (XANGLE)

CALL FEFLEC (REFLFX, ALFAN1, HETAN1, ANGINC, ANGREF, FOLAFI, SORINI)

REF $1=$ REFLFX

IF (IFFOGM,EQ . O) G0 TO 650

IF $(, J B E, E R, 0) G 0$ TO 602

IF (JBE, EQ, NA)

IF (LE,ER, O) MLE $=2$

IF $\{L B, E Q, 1) M L E=4$

GO TO 604

602 IF ( LE, EQ . O) MLE $=1$

IF ( $L E, E(R, 1) M L E=4$

60 TO 604

603 IF $(L E, E Q .0) M L B=1$ 
IF $(L E, E Q, 1) M L E=0$

604 FML =FLOAT (MLB)

$I F(J E E, E Q, N A, A N I, L E, E Q+1)$ GO TO 605

SMSUSF $=$ SMSUSF+FESUM1

SMSUSC=SMSUSC+CMSUM1

SMSURT $=$ SMSURT +RESUM2

SMSUCT = SMSUCT+CMSUM?

$605 \quad S M B=5 M B+F M L$ *GAUS*FEF 1

SMRG =SMEG +FML *GAUS

SMET = SMET +FML *GAUS*FEF2

650 CONTINUE

IF (IFFOGM , EQ + 0$) 60$ TO 600

$S \mathrm{H}=\mathrm{SE}+\mathrm{SME}$

SET $=$ SET +SMET

$S \mathrm{BG}=5 \mathrm{EG}+\mathrm{SMEG}$

SSUSF $=$ SSUSR+SMSUSR

SSUSC $=$ SSUSC + SMSUSC

SUSRT $=$ SUSFT TSMSURT

SUSCT $=$ SUSCT + SMSUCT

$600 \quad$ CONTINUE

IF (IFFOGM +EQ + O)GO TO 303

SUME $=H A * S B / 3$.

SUMET $=H A * S E T / 3$.

SUMEG $=H A * S B G / 3$.

FEF $(I Z)=S U M E /$ SUMBG

AFESUH $=$ SSUSF $/($ FNA $+1+$ )

ACMSUM $=$ SSUSC $/$ (FNA+1, )

REFT $(I Z)=$ SUMET/SUMEG

AFESUT $=$ SUSFT $/($ FNA 1 +)

ACMSUT $=$ SUSCT $/(\mathrm{FNAT} 1+\rangle$

303 IF (IFROGM +EQ . 0) ARESUM=FESUM1

IF (IFFOGM +EQ , O) ACMSUM =CMSUM1

IF (IFFOGM,ER, O)FEFT $(I Z)=F E F 2$

$I F(I F F O G M+E Q$, O) FEF $(I Z)=F E F 1$

C CALCULATION OF THE AUEFAGE REFFACTIVE INIEX ANI IIELECTRIC

C CONSTANT

CALL FEFINL (FEINIX, REFREX, REFCMX, ALFAX, BETAX, ARESUM,

\& ACMSUM, SOFINI , ANGINC, FAI)

FEINI $(I Z)=$ FEINIX

IIECM $(I Z)=$ KEFCMX

UIERE $(I Z)=$ FEFREX

$A L F A N=A L F A X$

BETAN=EETAX

IIEFTHN $(I Z)=$ REWAUE $/$ EETAN/FAI $/ 2$.

ANGLE2=SORSIN/FEINL (TZ)

ANGREN $(I Z)=180 . * A F S I N(A N G L E 2) / F A I$

IF (IFFOGM , ER . O) AFESUT =FESUMZ

IF (IFFOGM.EQ . O) ACMSUT $=$ CMSUM2

CALL REFINI (FEINIX, REFREX, REFCHX, ALFAX, EETAX, ARESUT,

\& ACMSUT, SORIND, ANGINC, F'AI)

FEINIT $(I Z)=$ FEINIIX

REFCMT $(I Z)=F E F C M X$

FEFFET $(I Z)=$ FEFFEX

ALFAT $=A L F A X$ 
EETAT $=$ BETAX

IIEFTH $(I Z)=$ REWAUE/BETAT $/ F A I / 2$.

ANGLE2 $=S O F S I N / R E I N I T(I Z)$

ANGFET (IZ) $=180$ * *AFSIN(ANGLE2)/FAI

CONT INUE

IF(LANGLE,GT + 0)GO TO 912

WRITE $(6,908)$

WRITE $(6,894)$

IF (IFROGM + ER + 0$)$ WRITE $(6,960)$

IF (IFFOGM, EQ+1) WRITE $(6,970)$

IF (IFOLA+LT+O)WFITE $(6,898)$

IF (IFOLA+GT, O) WFITE $(6,897)$

IF (IFOLA , ER , O) WFITE $(6,896)$

WRITE $(6,900)$ REWAUE, SF, GASIEN, VAPOR, TEMPER, HWI, EF OOP, HWN1, HWN2

WRITE $(6,907)$ AANGLE

908 FOFMAT $(1 H 1, / / / 23 X, " \odot * * *$ EXFERIMENTAL CONIITIONS $* * * \% * /)$

WFITE $(6.909)$

WFITE $(6,910)(Z(I Z)$, REFFET (IZ), REFCMT $(I Z)$, FEINDT $(I Z), \operatorname{IEPTH}(I Z)$,

\& ANGRET (IZ), REFT (IZ), IZ=1,IC)

910 FOFMAT (1H, $4 X, F 5,0,6 X, F 6,4,2 X, F 6,4,4 X, F 6,4,1 X, E B, 1,2 X, F 4,1$,

\& $3 X, F 6,4)$

WFI TE $(6,901)$ AANGLE

WFITE $(6,955)$

CALL GRAFH (IFFOGM ISINGLE, O, IC, IFREQ, FSC, REFRET)

WFI TE $(6,901)$ AANGLE

WRITE $(6,956)$

CALL GFAFH (IFFOGM, ISINGLE, O,IC, IFFEQ, FSC, REFCMT)

WFIT TE $(6,901)$ AANGLE

WFITE $(6,957)$

CALL GFAFH (IFROGM, ISINGLE, O,IC, IFRER,FSC, FEINDT)

WFI TE $(6,901)$ AANGLE

WFITE $(6,958)$

CALL GRAFH (IFFOGM, ISINGLE, O,IC, IFREQ,FSC,REFT)

912 WFITE $(6,908)$

WFIT TE $(6,895)$

IF (IFFOGM + ER + O) WFI TE $(6,960)$

IF (IFFOGM , ER . 1) WFI TE $(6,970)$

IF (IFOLA.LT.O) WFITE $(6,898)$

IF (IFOLA, GT, O) WFITE $(6,897)$

IF (IFOLA,EQ, O)WFITE $(6,896)$

WFITE $(6,900)$ FEWAVE, SF, GASIEN, UAFOF, TEMFEF, HWI, EFIOF, HWN1, HWN2

907 FOFIAT $(1 H 0,23 X, " * *$ ANGLE OF INCIDENCE*,IX,"=*,F4,1,"IIEGREE ***,1) WRITE $(6,909)$

901 FOFMAT $(1 \mathrm{H} 1, / / / 23 X, * *$ ANGLE OF INCTIENCE", $1 X, *=*, F 4,1, "$ IIEGREE , \& $* * * \% /)$

909 FOFMAT (1HO, "INCIIIENT FFEQ" $1 X$, "IITELECTFIC CONST" "2X, "FEFFACT" *

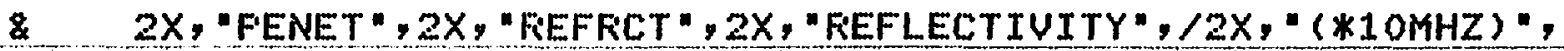

\& $6 X, " R E A L$ " $4 X, " I M A G ", 5 X$, INIEX", 4X, "IEFTH", 4X, "ANGLE", $/$ )

WRITE $(6,910)$ (Z(IZ), DIERE (IZ), DIECH (IZ), REINI (IZ), UEFTHN (IZ),

\& ANGREN (IZ), FEF (IZ), IZ=1,IC)

WFI TE $(6,901)$ AANGLE

WFIT TE $(6,955)$

CALL TFAFH(IFROGM, ISINGLE, 1, IC, IFFEQ,FSC, IIERE)

WFITE $(6,901)$ AANGLE 
WFITE $(6,956)$

CALL GFAFHSIFROGM, ISINGLE, 1 , IC, IFFEQ, FSC, IIECM

WFI TE $(6,901)$ AANGLE

WFITE $(6,957)$

CALL GFAFH (IFROGM, ISINGLE, 1, IC, IFREQ, FSC, FEINI)

WRITE $(6,901)$ AANGLE

WRIT TE $(6,958)$

CALL GRAFH IFROGM, ISTNGLE, 1, IC, IFRER, FSC, REF)

895 FORMAT (1HO,20X, "* WALL EFFECTS ARE TAKEN INTO ACCOUNT *\%")

894 FOFMAT $(1 H 0,20 \times$ * * WALL EFFECTS ARE NOT TAKEN INTO ACCOUNT *\%")

955 FOFMAT 1 HO " SFFEQ " $34 \times, " \angle F E A L$ FAFT OF THE IIELECTFIC",

\& CONSTY")

956 FOFMAT (1HO, "FRER\%",34X, "IMAG FART OF THE IIELECTKIC",

\& "CONST")

957 FOFMAT (1HO, "FFEQ\%, 34X, "REFFACTIUE INIEX\%")

958 FOFIMAT (1HO, "FFER\%", 34X, "FEFLECTIUITY?")

960 FORMAT (4X, "WHITE LIGHT IS INCIIENT")

970 FORMAT $4 X$, "GAUSIAN BEAM WITH A NAFROW LINE WIITH IS IN",

\& "CIDENT \& SCANNEI")

$300 \quad$ CONTINUE

STOF

ENI!

C SUBFOUTINE TO CALCULATE SUSCEPTIUILITY FOR THE BULK GAS

SURROUT INE SUSCEP (FESUM,CMSUM,N,Z,H,XALLX,SF,CONST,

\& 0S1, 0S2, [1. HW HW1, HWN2, ILINE)

ZEFO $=0$.

IF $(0 S 1$ - EQ , ZEFO + ANI + ILINE +EQ + 1)GO TO 12

IF (OS2,EQ + ZEFO +AND, ILINE,EQ + 2)GO TO 12

IF (ILINE, EQ + 1) HWNX $=$ HWNI

IF (ILINE,EQ+2) HWNX=HWN2

IF (ILINE, EQ +1) $05 X=051$

IF (ILINE, EQ + 2) OSX $=052$

$I F(I L I N E \cdot E Q+2) Z=Z-S F$

SFAA $=0$.

$S C A A=0$.

Nio $10 \quad J=1, N+1,2$

$J L=\sqrt{ }=1$

II $=H$ H*FLOAT ( JIL) +XALLX

SF:AAA $=0$.

SCAAA $=0$.

tio $5 \quad L=1,2$

$M=L-1$

IIM $=F$ LOAT $(M)$

$X A=I I+H *$ IIM

$A 11=4+*(X A * * 2) /([1 \cup * * 2)$

$A 1=-A 11$

BIU $=0$ SX*EXF'(A1)

$F A A=(X A-Z) * * 2+H W N X * * 2$

$F A=B 1 U / F A A$

REFA $=(X A-Z) * F A$

CMFA $=H W N X * F A$

IF $(\Omega L, E Q+O)$ GO TO 2 
IF (JL.EQ.N) GO TO 3

IF $(M, E Q, 0) \quad M L=2$

IF $(M, E Q, 1) \quad M L=4$

GO TO 4

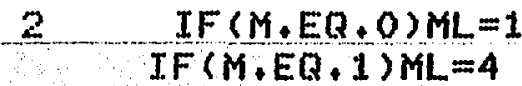

Go To 4

$3 \quad \quad I F(M+E Q, O) M L=1$

$I F(M, E Q, 1) M L=0$

$4 \quad$ IIMLL=FLOAT (ML)

SRAAAA $=S R A A A A+$ IML $* F E F A$

SCAAA $=$ SCAAA+TIML $* C M F A$

5 CONTINUE

SRAA $=$ SR $A A+S R A A A$

SCAA $=$ SCAA+SCAAA

10 CONTINUE

RESUM $=$ CONST $* H * S R A A / 3$.

CMSUM $=$ CONST $* H * S C A A / 3$.

$I F(I L I N E+E R+2) Z=Z+S F$

GO TO 13

12 RESUM $=0$.

CMSU $=0$.

13 RETURN

ENI

C SURROUTION TO GRAFH IIATA

SUEROUTINE GRAFH(IFROGM, ISINGLE, IWALL, IC, IFFER, FSC, VALUE)

MIMENSION FLOT (83), VALUE(TC)

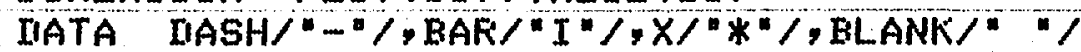

no $5 I=1$ \& IC

IF (I.EQ.1) YMAX $=$ VALUE (I)

$I F(I, E Q, 1) Y M I N=U A L U E(I)$

$I F(I+G E, 2)$ GO TO 2

GO TO 5

2 BIG $=$ VALUE $(I)-Y M A X$

SMALL $=$ VALUE (I) - YMIN

$Z E F O=0$.

IF ( $B I G+G E, Z E F O)$ YMAX $=U A L U E(I)$

IF (SMALL,LT,ZEFO) YMIN=UALUE(I)

$5 \quad$ CONTINUE

IIIF $=Y M A X-Y M I N$

no $10 \quad K=1,83$

FLOT $(K)=$ IIASH

10 CONTINUE

WRITE $(6,20)$ FLOT

20 FOFMAT $(1 X, "(* 10 M H Z) ", / 1 \mathrm{HO}, 15 X, 83 \mathrm{~A} 1)$

no $30 \quad k=1,83$

FLOT $(K)=$ FLANK

30 CONTINUE

FLOT $(1)=B A R$

II0 $60 \quad K Z=1, I C$

$K Z Z=K Z Z-1$

$Z=-F L D A T(I F R E Q)+F L O A T(K Z Z) * F S C$ 
$Y \Pi I F=U A L U E(K Z)-Y M I N$

$Y N=Y$ IIF $* 80, /$ IIF +2.5

$N Y=I F I X(Y N)$

FLOT $(N Y)=X$

WFITE $(6,40) Z$, VALUE $(K Z)$, FLOT

40 FOFMAT $(1 X, F 5,0,2 X, F 6,4,1 X, 83 A 1)$

FLOT $(N Y)=B L A N K$

60 CONT INUE

IF (IFROGM,EQ , O) WFITE $(6,71)$

IF (IFFOGM + EQ + 1 ) WRITE $(6,72)$

IF (ISINGLE+EQ+3) WFITE $(6,61)$

IF (ISINGLE, EQ + I) WFI TE $(6,64)$

IF (ISINGLE,EQ + 2) WFITE $(6,65)$

IF (IWALL,EQ + 1) WRITE $(6,62)$

IF (IWALL, EQ, O)WRITE $(6,63)$

64 FOFMAT (1HO, * THE CENTFAL FFEQ OF F=2 LINE IS AT $0 . \%$ "

\& "F=1 LINE IS NOT FRESENT")

65 FOFMAT (1HO, * THE CENTFAL FREQ OF F=1 LINE IS AT 177.2 \&

\& " $F=2$ LINE IS NOT PRESENT")

61 FORMAT 1 1HO "* THE CENTFAL FFEQ OF ONE OF 02 LINES "

\& (F=2) IS AT FRER=0, WHEREAS THE OTHER (F=1) IS AT 177.2 ",

8 * *)

62 FOFMAT(1HO, * THE WALL EFFECT IS TAKEN INTO ACCOUNT * *)

63 FOFIMAT 1 1HO, * THE WALL EFFECT IS NOT TAKEN INTO "

\& "ACCOUNT *\%

71 FORMAT (1HO, * WHITE LIGHT IS INCIIENT **)

72 FOFMAT 1 HO, * GAUSIAN REAM WITH A NARROW LINE WITITH IS INCI",

$\&$ "IIENT \& SCANNEI *")

FETUFiN

ENII

C SUEROUTINE TO CALCULATE SUSCEFTIEILITY FOF THE GAS NEAR THE SURFACE

SUBFOUT INE SUSCEF(FESUM, CMSUM, N1, N2, Z,H, XLOWAL, SF,

\& C, OS1, OS2, IIF, HWN1, HWN2, DOFS, ALFAZT, HETAZT,

\& LANGLE , ANGINC Y ANGFEF, NY,HY, ILINE)

C SRF $=0$. ANI SCF $=0$. ARE INITIALTZATIONS TO TAKE UELOCITY AUERAGE

C W.F.T THE $\times$ COMFONENT OF THE IIOFFLER SHIFTEN FRER

ZEFO=0.

IF (OS1,EQ + ZEFO + AND + ILINE +ER \& 1)GO TO 33

IF (OS2, EQ + ZEFO + ANI. ILINE +EQ . 2)GO TO 33

CFIT TAN $=80$.

IF ( ILINE,ER + 1$) 0 S X=0 S 1$

IF (ILINE, ER. I)HWNX=HWN1

IF (ILINE, EQ + 2) OSX $=0.52$

IF (TLINE.EQ. 2)HWNX=HWN2

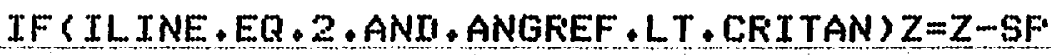

IF ( IL INE, ER, I) N=N1

IF (IL INE, ER, 2 ) N=N2

IF (LANGLE,EQ. O)GO TO 1

$S F F=0$.

$S C F=0$.

$1 \quad I F$ (LANGLE, ER + O)NY $=0$

IID $20 \mathrm{IY}=1, N Y+1,2$ 
IF (LANGLE.EQ.O) GO TO 2

$I Y Y=I Y-1$

$\Pi Y=H Y * F L O A T(I Y Y)-0,8 * I O F G$

SFIF $Y=0$.

SCFY $Y=0$.

LL $2=2$

2 IF (LANGLE,EQ , O) LL2=1

IIO $15 \quad L L=1, L L 2$

IF (LANGLE,EQ,O)GO TO 5

$M M=L L-1$

FMM $=$ FLOAT ( $M M$ )

$Y 1=n Y+H Y * F M M$

AY $1=-4 * *(Y 1 * * 2) /(\operatorname{IOF} 5 * * 2)$

EAY $1=E X P(A Y 1)$

$5 \quad$ IF (LANGLE,EQ 0$) Y 1=0$.

IF (LANGLE, ER + O) EAY $1=1$ +

SREAR $=0$.

SCHAB $=0$.

IF (ANGREF , GT, CRITAN) $N=1$

IIO $16 \quad L A=1, N+1,2$

IF (ANGREF, GT, CRITAN) GO TO 3

LL. $A=L A-1$

IIAL =H*FLOAT (LLA) +XLDWAL

SFIFAE $=0$.

$S C F A B=0$.

$I I 2=2$

3 IF (ANGFEF, GT, CFITAN) I I $2=1$

IIO 17 II $=1$, IIZ

IF (ANGFEF . GT + CFITAN) GO TO 6

$N N=I I-1$

$F N N=F L O A T(N N)$

$A L=M A L+H * F N N$

IF $(L A N G L E+E Q, 0) Y 1=0$.

MOFC $=$ IF'*ALFAZT

$A L L 1=4, *(A L * * 2) / \operatorname{LOFC} * * 2$

IF (ALL1 +GT + 60$) G 0$ TO 42

EAL 1 $=\operatorname{EXF}(-A L L 1)$

GO TO 7

$42 \quad$ EAL $1=0$.

GO TO 7

$6 \quad E A L 1=1$.

$A L=0$.

$A L Z 1=A L+Y 1-Z$

$A L Z 2=A L+Y 1-Z+5 F$

$A L W=B E T A Z T * A L / A L F A Z T$

$A L W 1=A L W-H W N 1$

ALW2 $=A L W-H W N 2$

ALZ12=ALZ1**2

$A L Z 22=A L Z 2 * * 2$

AL W12=ALW1 $* * 2$

ALW22=ALW2**2

ALX1 $=A L Z 12+A L W 12$

$A L X 2=A L Z 22+A L W 22$

IF (LANGLE,ER, 0)EAY $1=1$. 
RFAL 1 =EAY $1 * E A L 1 * 051 * A L Z 1 / A L X 1$

FIFAL 2=EAY $1 * E A L 1 * 052 * A L Z 2 / A L \times 2$

CFAL $1=-E A Y 1 * E A L 1 * A L W 1 * 0 S 1 / A L X 1$

CFAL2=-EAY 1*EAL 1 *ALW2*052/ALX2

GO TO 8

AL: $Z 1=A L+Y 1-Z$

ALW $=E E T A Z T * A L / A L F A Z T$

$A L W X=A L W-H W N X$

ALZ12=ALZ1**2

AL.W12=ALWX**2

$A L X 1=A L Z 12+A L W 12$

IF (LANGLE+EQ + O) EAY $1=1$.

FFAL $1=E A Y 1 * E A L 1 * 0 S X * A L Z 1 / A L X 1$

CFAL 1 =-EAY $1 * E A L 1 * A L W X * 0 S X / A L X 1$

IF (LLA.EQ. O)GO TO 12

IF (LLA.EQ N N)GO TO 13

$I F(N N, E Q, O) N C=2$

$I F(N N+E Q+1) N C=4$

GO TO 14

$12 \quad$ IF $(N N+E Q+O) N C=1$

$I F(N N, E Q, 1) N C=4$

GO TO 14

13 IF $(N N, E Q, 0) N C=1$

$I F(N N \cdot E Q+1) N C=0$

$14 \quad F N C=F L D A T$ (NC)

SFFAB = SFFAB+FNC $*$ RFAL 1

SCFAB = SCFAE +FNC*CFAL1

17 CONTINUE

SF:EAB=SFFAB+SFEAE

SCMAE = SCMAB + SCKAB

16 CONTINUE

FUNFEY $=H * S F E A E / 3$.

FUNCMY $=H * S C M A B / 3$.

8 IF ( ANGFEF, GT , CFITAN) FUNFEY =FIFAL 1+FIFAL2

IF (ANGFEF , GT , CFI I TAN ) FUNCMY =CFAL1 +CFAL2

IF (LANGLE+EQ +O) G0 TO 31

IF (IYY,EQ,O)GO TO 22

IF (IYY,EQ.NY)GO TO 23

$I F(M M, E Q, O) M C=2$

$I F(M M+E Q+1) M C=4$

GO TO 24

22 IF $(M M, E Q \cdot O) M C=1$

$I F(M M+E Q+1) M C=4$

$60 \mathrm{TO} 24$

$23 \quad I F(M M+E Q+O) M C=1$

$I F(M M+E Q+1) M C=0$

$24 \quad F M C=F L O A T$ (MC)

SRFY $=$ SRRY +FMC $* F U N R E Y$

SCRY = SCFY +FMC *FUNCMY

15 CONTINUE

$S R F=S F R+S R F Y$

SCF $=$ SCF + SCFY

20 CONTINUE

RESUM=C*HY $* S R K / 3$. 
CMSUM $=\mathrm{C} * H Y * S \mathrm{CF} / 3$

60 TO 32

31 FESUM $=$ C*FUNREY

CMSUM $=$ C*FUNCMY

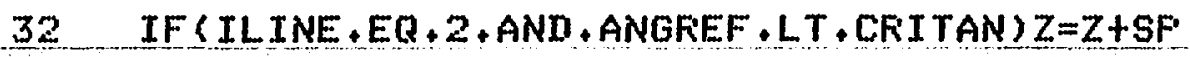

$33 \quad$ RO TO 34

CNSUM $=0$.

34 FE.TUFN

ENII

C SURFOUTINE TO CALCULATE REFFACTIUE INIEX

SURFOUTINE REFINI SFEINIX, FEFREX, REFCMX, ALFAX, EETAX,

8 SUSFEX, SUSCMX, SOINIX,ANGINC, PAI)

FEFFEX $=1++4$, *FAI*SUSFEX

FEFCMX $=2$ * *FAI*SUSCMX

FAFTI $=($ SOINIX*SIN(ANGINC) $) * * 2$

$A=$ FEFFEX - FAFITI

$B=2$ * *FEFCMX

C=FIEFFEX+FART1

$\eta l=0$.

$A B=A E S(A)$

IF $(A B-B) 60,61,62$

60 $\quad X=A / E$

$X F i T=1+X * * 2$

$A L F A=E *(S R R T(X F T)+X) / 2$.

ALFAX $=5 Q R T$ (ALFA)

BETA $=\mathrm{E} *(\mathrm{SQR} T(X \mathrm{XFT})-X) / 2$.

$E=S R F T$ (XFT) $-X$

IF ( $E+E Q$, II) BETAX $=0$.

BETAX $=$ SQRT ( BETA)

FAFT $X=(C+E * X R T) / 2$.

REINIIX=SQFT (FARTX)

00 To 63

$61 \quad$ ALFA $=B *(1++\operatorname{SQFT}(2+)) / 2$.

ALFAX $=$ SQRT (ALFA)

EETA $=E *(\operatorname{SQRT}(2+)-1) /$,2 .

BETAX =SQRT (BETA)

FAFTE $=(C+E *(1,+\operatorname{SQFT}(2))) /$,2 +

FEINIX $=$ SQRT (FARTE)

GD TO 63

$62 \quad Y=B / A$

$Y F T=1+Y * * 2$

$A L F A=A *(1++S Q R T(Y R T)) / 2$

IF (A.LT, I ) ALFA=AB* (SRFT (YFT $)-1+) / 2$.

ALFAX $=$ SQFT $(A L F A)$

BETA $=A *(S Q R T(Y R T)-1) /$,2 .

IF (A.LT,II)EETA $=A B *($ SQRT $(Y F T)+1) /$,2 .

$F=S R R T$ ( YFT $)-1$.

$I F(F, E Q+$ II) BETAX $=0$.

BETAX $=$ SQRT (BETA)

FARTY $=(C+A * Y R T) / 2$.

FEINIX $=S Q R T$ (FARTY) 
$64 \times$ XC1=REFCMX**2/(FAFT1-REINNX**2)

$X C 2=X C 1+F A R T 1$

$X C=S Q R F T(X C 2)$

REINIIX $=X C$

65 RETUFIN

ENI

C SUEFOUTINE TO IEFINE A FUNCTIONAL FORM OF REFLECTIUTTY

SUEROUTINE FEFLEC (REFLFX, ALFAX, BETAX, ANGINC,

\& ANGFEF, FOLAFI, SOINIX)

$A C=\operatorname{COS}(A N G I N C)$

$A S=S I N(A N G I N C)$

RNUMAX $=(S O I N I X * A C-A L F A X) * * 2+$ EETAX**2

FIEND $=(S O I N I X * A C+A L F A X) * * 2+$ HETAX**2

$S A=S O I N I X * A S$

$X A=(A L F A X * * 2-B E T A X * * 2+S A * * 2) * A C$

$X A N=X A-S O I N I X * A L F A X$

$X E=2+* A L F A X * B E T A X$

$X$ XN $=X B-$ SDINIIX $* B E T A X$

FNFEF $=X A N * * 2+X E N * * 2$

$X A I=X A+S O I N U X * A L F A X$

$X \mathrm{BI}=X \mathrm{XB}+50 \mathrm{INIX} \times \mathrm{BETAX}$

RIFER $=X A T * * 2+X \mathrm{BI} * * 2$

IF (FOLAFI) $701,702,703$

701 FEEILFX=FNPEF/FIIFER

GO TO 705

703 REFLFX $=$ FINUMAX/FIMENO

60 ro 705

702 FEFLFX $=.5 *$ (FNFER/FMFEF +FNUMAX/RIEND)

705 FETUFN

END

C. SUEFOUTINE TO FINI THE SRUAFE OF THE INCIIIENT LIGHT AMFLITURE

SUBROUTINE GAUSTA (AIGAUS, XINC, AAZ, HWI, FAT)

$A Z=A A Z-X T N C$

$A I G=-(A Z * * 2) / H W I / H W I$

AIGAUS $=E X F(A I G)$

RETUFIN

ENII

EXECUTE

LIMITS $40,47 K, 5000$

\$ SYSOUT OG. OFG

ENIJUE 
$<\star \star \star$ EXPERIMENTAL CONDITIONS $\star \star \star\rangle$

GAUSIAN BEAM WITH A NARROW LINE WIDTH IS INCIDENT \& SCANNED

POLARIZATION IS PERPENDICURAR

RESONANCE WAVE LENGTH OF NA DZ LINE

$$
=0.589 E-04 \text { (CM). }
$$

SPגLITTING OF HYPERFINE DOUBLET AT GRAND STATE

$$
=177.2 \quad(\neq 10 \mathrm{MHZ})
$$

DENSITY OF VAPOR $=0.105 E 16$ (PER CUBIC CM)

VAPOR PRESSURE $=0.067$ (MMHG)

TEMPERATURE OF GAS $=619$.

HALF OF $1 /$ POINTS $S^{\circ}$ WIDTH OF INCIDENT LIGHT

$$
=24.0 \quad(\star 10 \mathrm{MHZ})
$$

1/E POINTS! DOPPLER WIDTH

$$
=227.2(\star 10 M H Z)
$$

HALF OF HALF WIDTH OF PRESSURE BROADENED LINE 1

$$
=4.3 \ldots \text { (*1.MHZ) }
$$

HALF OF HALF WIDTH OF PRESSURE BROADENED LINE 2

$=2.9(\star 1$ OMHZ $)$

* ANGLE OF INCIDENCE $=0$. DEGREE * *

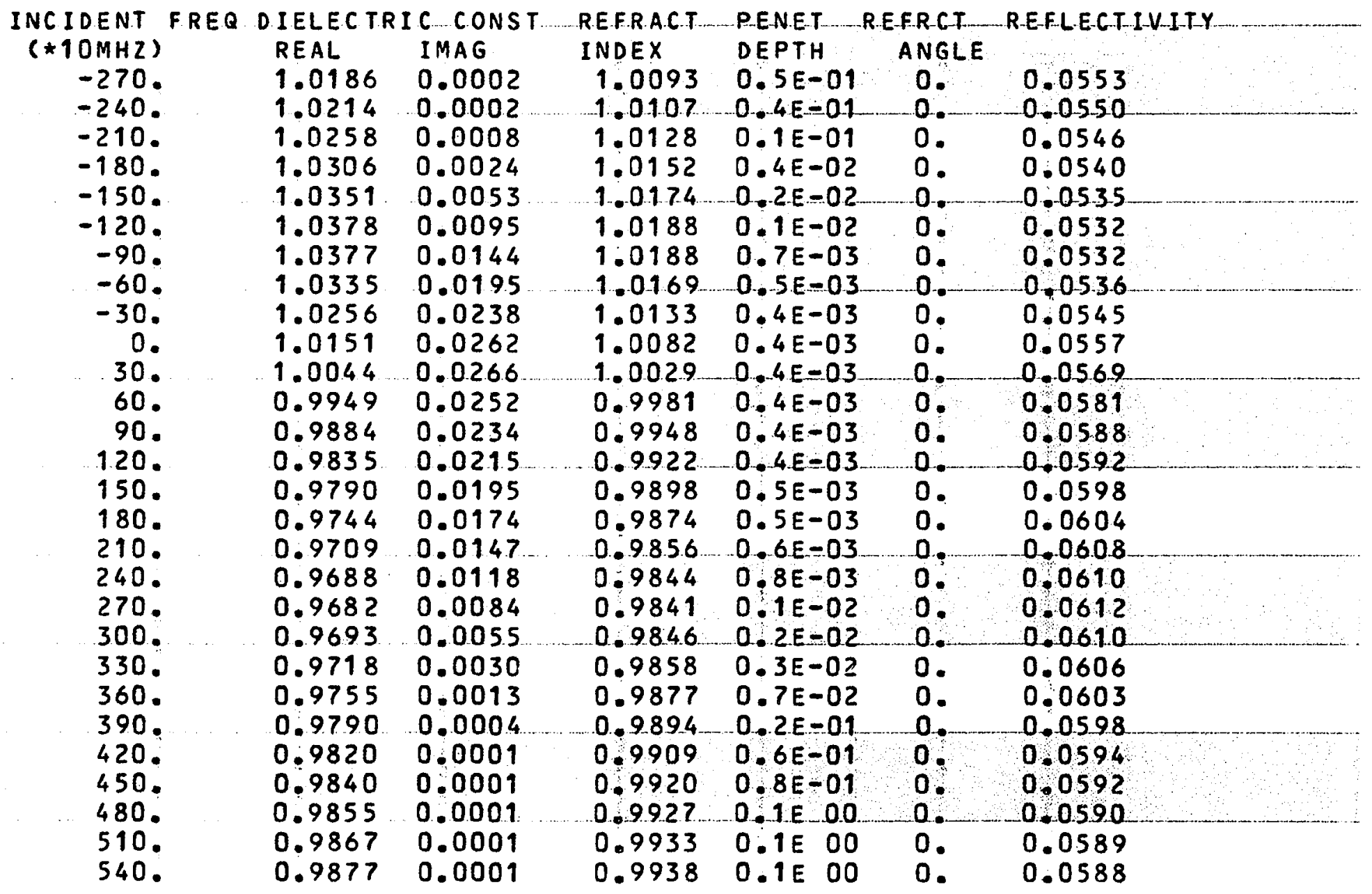




* ANGLE OF INCIDENCE $=0$. DEGREE * $*$

(F.R.EQS

$(\star 1$ OMHZ)

〈REELEECTIVITY)

$-270.0 .0553$

$-240.0 .05501$

$-210 . \quad 0.0 .546$

$-180.0 .05401$

-150.0 .0535 I

-120.0 .0532 I*

$-90.0 .0532 \mathrm{I}$ *

$-60.0 .05361$

$-30 . \quad 0.054 .5$

0.0 .05571

30. 0.05691

60. $0.0581 \mathrm{I}$

90. $\quad 0.0588 I$

120. $0.0592 \mathrm{I}$

$150 \ldots .0 .0 .598$

180. 0.06041

210. 0,06081

240. 0.06101

270. 0.0612 I

300. 0.06101

$330 . \quad 0.06061$

360. 0.06031

390.0 .0598

4.20 .0 .0 .5941

450. 0.0592 I

480.0 .05901

$510 \ldots 0.05891$

$540.0 .0588 \mathrm{I}$

$\star$

* GAUSIAN BEAM WITH A NARROW LINE WIDTH IS INCIDENT \& SCANNED *

* THE CENTRAL FREQ OF ONE OF D2 LINES (F=2) IS AT FREQ=0. WHEREAS THE OTHER (F=1) IS AT 177.2 * * the wall efFect is nOt taken into account * 


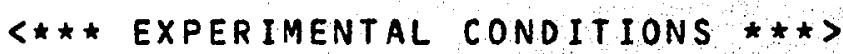

\section{<* WALL EFFECTS ARE TAKEN INTO ACCOUNT $\star>$}

GAUSIAN BEAM WITH A NARROW LINE WIDTH IS INCIDENT \& SCANNED

POLARIZATION IS PERPENDICURAR

RESONANCE WAVE LENGTH OF NA D.2 L.INE

$$
=0.589 E-0.4 \text { (CM) }
$$

SPXLITTING OF HYPERFINE DOUBLET AT GRAND STATE

$$
=1772 \quad(* 10 \mathrm{MHZ})
$$

DENSITY OF VAPOR $=0.105 E 16$ (PER CUBIC CM)

VAPOR PRESSURE $=0.067$ (MMHG)

TEMPERATURE OF GAS $=619$.

HALF OF $1 /$ E POINTS ' WIDTH OF INCIDENT LIGHT

$$
=24.0 \quad(* 10 \mathrm{MHZ})
$$

$1 /$ E POINTS DOPPLER WIDTH

$$
=227.2(\star 10 \mathrm{MHZ})
$$

HALF OF HALF WIDTH OF PRESSURE BROADENED LINE 1

\begin{tabular}{|c|c|c|c|c|c|c|}
\hline 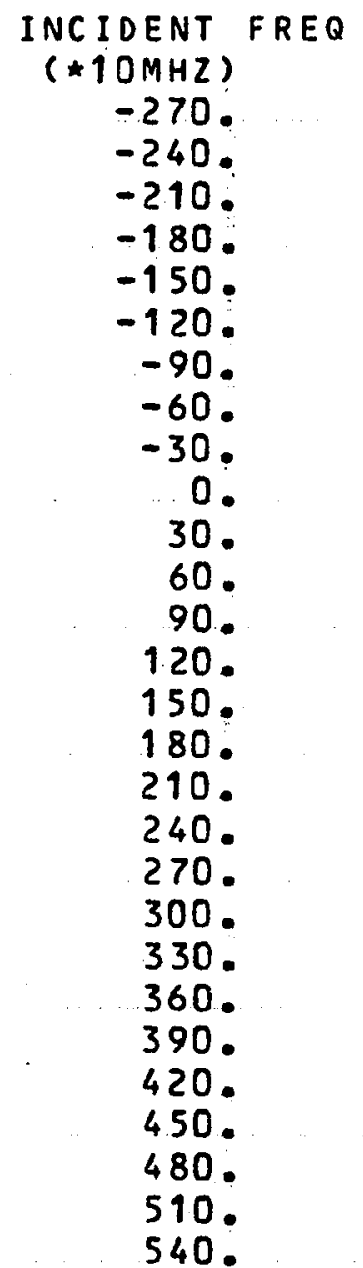 & $\begin{array}{l}\text { DIELECTR } \\
\text { REAL } \\
1.0187 \\
1.0215 \\
1.0259 \\
1.0307 \\
1.0348 \\
1.0359 \\
1.0336 \\
1.0269 \\
1.0156 \\
0.9979 \\
0.9828 \\
0.9783 \\
0.9837 \\
0.9884 \\
0.9849 \\
0.9763 \\
0.9658 \\
0.9603 \\
0.9604 \\
0.9661 \\
0.9715 \\
0.9757 \\
0.9791 \\
0.9820 \\
0.9840 \\
0.9855 \\
0.9867 \\
0.9877\end{array}$ & $\begin{array}{l}\text { IC CONST } \\
\text { IMAG } \\
0.0002 \\
0.0002 \\
0.0008 \\
0.002 .5 \\
0.0055 \\
0.0095 \\
0.0143 \\
0.0192 \\
0.0235 \\
0.0258 \\
0.0209 \\
0.0142 \\
0.0099 \\
0.0121 \\
0.0143 \\
0.0149 \\
0.0128 \\
0.0096 \\
0.0061 \\
0.0042 \\
0.0029 \\
0.0013 \\
0.0004 \\
0.0001 \\
0.0001 \\
0.0001 \\
0.0001 \\
0.0001\end{array}$ & $\begin{array}{l}\text { REFRACT } \\
\text { INDEX } \\
1.0093 \\
1.0107 \\
1.0129 \\
1.0153 \\
1.0173 \\
1.0179 \\
1.0 .169 \\
1.0137 \\
1.0083 \\
0.9996 \\
0.9918 \\
0.9893 \\
0.991 .9 \\
0.9943 \\
0.9926 \\
0.98 .83 \\
0.9829 \\
0.9800 \\
0.9800 \\
0.9829 \\
0.9856 \\
0.98 .98 \\
0.9895 \\
0.9910 \\
0.9920 \\
0.9927 \\
0.9933 \\
0.9938\end{array}$ & $\begin{array}{l}\text { PENET } \\
D E P T H \\
0.5 E-01 \\
0.4 E-01 \\
0.1 E-01 \\
0.4 E-0.2 \\
0.2 E-02 \\
0.1 E-02 \\
0.7 E-0.3 \\
0.5 E-03 \\
0.4 E-03 \\
0.4 E-0.3 \\
0.4 E-03 \\
0.7 E-03 \\
0.9 E-03 \\
0.8 E-03 \\
0.7 E-03 \\
0.6 E-03 \\
0.7 E-03 \\
0.1 E-02 \\
0.2 E-02 \\
0.2 E-02 \\
0.3 E-02 \\
0.7 E-02 \\
0.2 E-01 \\
0.6 E-01 \\
0.8 E-01 \\
0.1 E 00 \\
0.1 E 00 \\
0.1 E-00\end{array}$ & 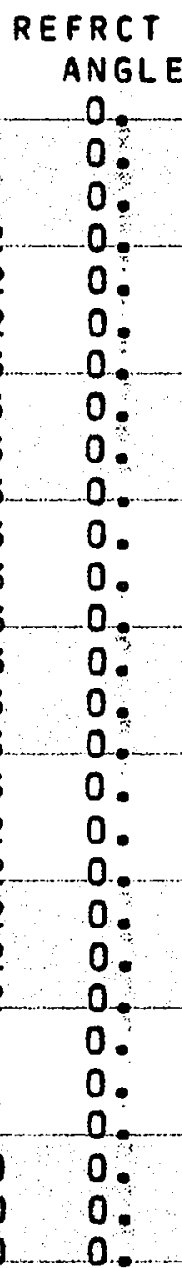 & $\begin{array}{l}0.0553 \\
0.0550 \\
0.0546 \\
0.0539 \\
0.0535 \\
0.0534 \\
0.0536 \\
0.0543 \\
0.0555 \\
0.0574 \\
0.0599 \\
0.0604 \\
0.0588 \\
0.0585 \\
0.0590 \\
0.060 .0 \\
0.0614 \\
0.0624 \\
0.0622 \\
0.0612 \\
0.0606 \\
0.0602 \\
0.0597 \\
0.0594 \\
0.0592 \\
0.0590 \\
0.0589 \\
0.0588\end{array}$ \\
\hline
\end{tabular}
$=4.3$. $\quad(* 1 . \mathrm{MHZ})$

HALF OF HALF WIDTH OF PRESSURE BROADENED LINE 2 $=2.9 \quad(* 10 \mathrm{MHZ})$ 
* ANGLE OF INCIDENCE $=0$. DEGRE * *

〈FR.EQ

<REAL PART OF-THE-DIELECTRICCONSTS

$(\star 10 M H Z)$

$-270 . \quad 1.01871$

$-240.1 .02151$

$-210.1 .02591$

$-180.1 .03071$

$-150.1 .03481$

$-120,1.03591$

$-90 . \quad 1.03361$

-60.1 .0269 I

$-30 . \quad 1.0156 I$

0.0 .99791

30. 0.9828 I

$60 . \quad 0.97831$

90. 0.9837

$120,0.9884$ I

$150 . \ldots .9849$ I

180. 0.9763 I

210. 0.9658 I

240. 0.96031 *

270. 0.9604 I

300. $0.9661 \mathrm{I}$

$330, \quad 0.9715 \ldots$

$360: 0.9757$ I

390. 0.9791 I

$420 . \quad 0.9820$ I

450. $0.9840 \mathrm{I}$

480. 0.9 .8551

$510,0.9867 \ldots$

540. 0.9877 I

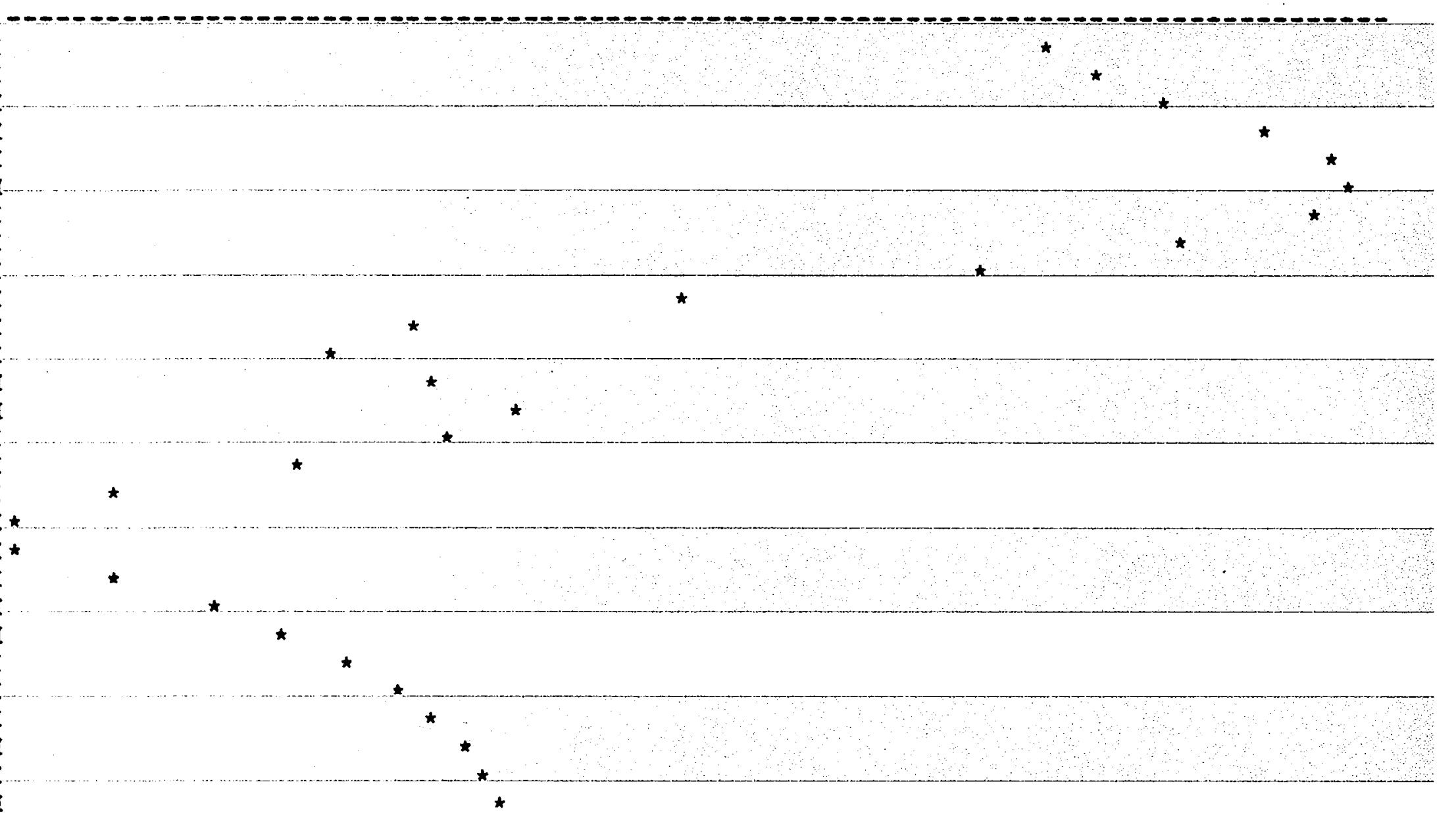

* gaUSian beAM WITH a NARROW LINE WIDTH IS INCIDENT \& SCANNED *

* THE CENTRAL FREQ OF ONE OF O2 LINES (F=2) IS AT FREQ=0. WHEREAS THE OTHER (F=1) IS AT 177.2 *

* THE WALL EFFECT IS TAKEN INTO ACCOUNT * 
* ANGLE OF INCIDENCE $=0$. DEGREE $\star$

$-270.0 .00021 *$

$-240.0,00021 *$

$-210 . \quad 0.0 .008 \ldots \star 1 \ldots$

-180.0 .0025 I

-150.0 .0055 I

$-120 . \quad 0.009 .51$

-90.0 .0143 I

-60.0 .0192 I

$-30.0 .02351$

$0.0 .0258 \mathrm{I}$

30. 0.0209 I

60. $0.01 .42 \mathrm{I}$

90. 0.00991

120. $0.0121 \mathrm{I}$

150. $0.0143 \mathrm{I}$

180. 0.0149 I

210. 0.0128 I

240. $0.0096 \mathrm{I}$

270. $0.0061 \mathrm{I}$

300. 0.0042 I

330. $\quad 0.00291$

360. 0.0013

390. 0.00041 *

4.20. 0.0001 I*

450. 0.0001 i*

480. 0.0001 i*

510. 0.0001 I*

540. 0.0001 I*

^ GAUSIAN BEAM WITH A NARROW LINEWIDTH IS INCIDENT \& SCANNED *

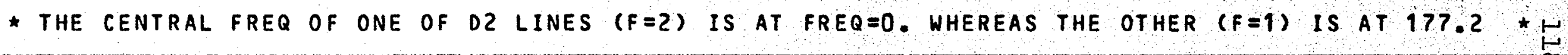

* The hall efFect is taken into acCOUNT * 
$\star$ ANGLE OF INCIDENCE $=0$. DEGREE *

SFR.EQS

$(* 10 M H Z)$

SREFLECTIVITYS

$-2.70 . \quad 0.0553$

$-240.0 .05501$

$-210.0 .05461$

-180.0 .0539 I

$-150.0 .0535 \mathrm{I}$ *

$-120 . \quad 0.0 .53 .4 \ldots$

$-90 . \quad 0.05361$

$-60.0 .05431$

$-30 . \quad 0.0 .5551$

0.0 .05741

30: 0.0 .599 .1

60. 0.060 .1

90. $0.0588 \mathrm{I}$

120. 0.05851

1.50. $\quad 0.0 .5901$

180. 0.06001

210. 0,06141

240. 0.0624

270. $\quad 0.06221$

300. 0.06121

330. 0.0.606 I

360. 0.06021

390. 0.0597 I

4.20. $\quad 0.0594 \ldots$

450.0 .0592 I

480. 0.05901

510.0 .05891

540. $0.0588 \mathrm{I}$

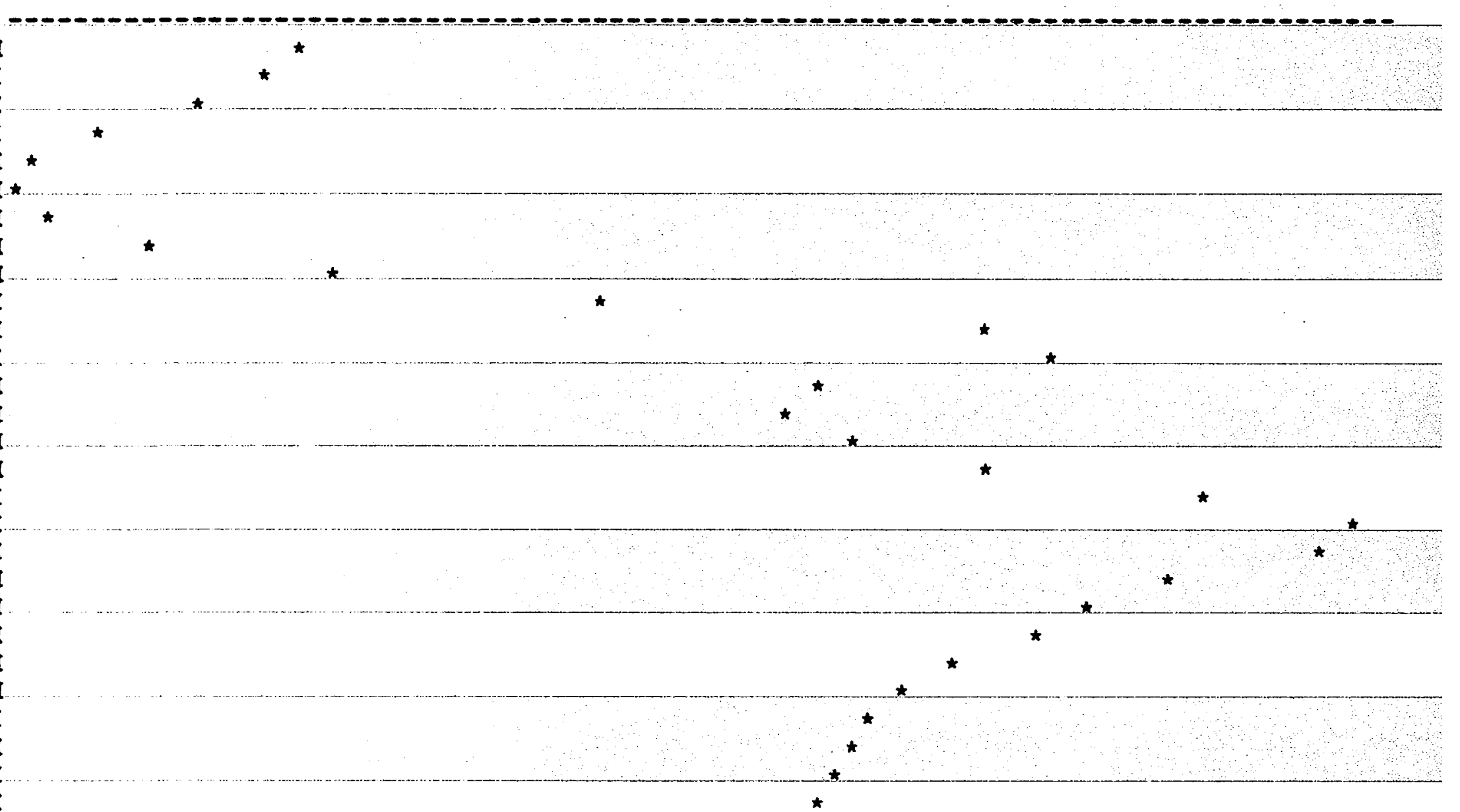

* GAUSIAN BEAM WITH A NARROW LINE WIDTH IS INCIDENT \& SCANNED *

* THE CENTRAL FREQ OF ONE OF D2 LINES (F=2) IS AT FREQ=0. WHEREAS THE OTHER (F=1) IS AT 177.2 * 2

* THE WALl EFFECT IS TAKEN INTO ACCOUNT * 
\&* WALL EFFECTS ARE TAKEN INTO ACCOUNT $*$ >

GAUSIAN BEAM WITH A NARROW LINE WIDTH IS INCIDENT \& SCANNED

POLARIZATION IS PERPENOICURAR

RESONANCE WAVE LENGTH OF NA D.2 LINE

$$
=0.589 E-04 \quad(C M)
$$

SPXLITTING OF HYPERFINE DOUBLET AT GRANO STATE

$$
=177.2 \quad(* 1 . \mathrm{MHZ})
$$

DENSITY OF VAPOR $=0.105 E 16$ (PER CUAIC (M)

VAPOR PRESSURE $=0.067$ (MMHG)

TEMPERATURE OF GAS = 619.

HALF OF 1/E POINTS " WIOTH OF INCIDENT LIGHT

$$
=24.0 \quad(* 10 \mathrm{MHZ})
$$

1 /.E POINTS' DOPPLER W.I.DTH

$$
=227.2(\star 10 \mathrm{MHZ})
$$

HALF OF HALF WIDTH OF PRESSURE BROADENED LINE 1

$=4.3 \quad(* 1 . M H Z)$

HALF OF HALF WIDTH OF PRESSURE BROADENED LINE 2

$=2.9 \quad(* 10 \mathrm{MHZ})$

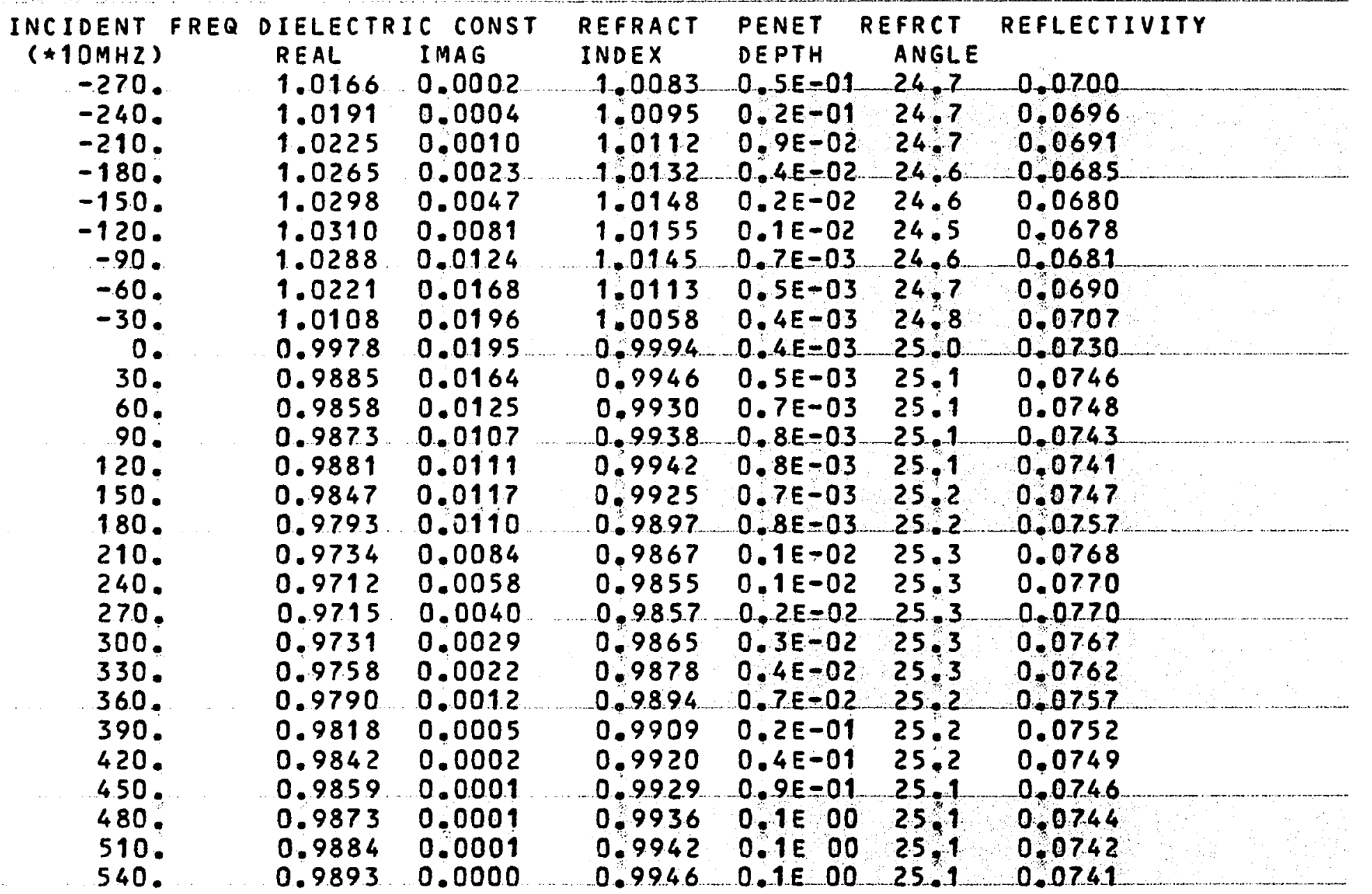



$\star \star A N G L E$ OF INCIDENCE $=15.0$ DEGRE $\star \star$

CFREQS

CIMAG PART OF THE DIELECTRICCONSTS

$(\star 10 M H Z)$

-270.0 .00021 .

$-240.0 .00041 \star$

$-210 \ldots 0.001 .0 \ldots$

$-180.0 .00231$

-150.0 .0047 I

$-120 \ldots 0.0081 \ldots 1$

$-90.0 .0124 I$

$-60.0 .01681$

$-30.0 .01961$

0.0 .01951

$30.0 .0164 \mathrm{I}$

$60.0 .0125 \ldots 1$

90. 0.0107 I

120. 0.0111 I

150. 0.01171

1.80. 0.01101

210. $0.0084 \mathrm{I}$

2.4.0. $0.0058 \ldots$

270. $0.0040 \mathrm{I}$

300.0 .00291

330. 0.00221

360. 0.0012 I

390. 0.00051

420. $0.00021 \star$

450. 0.00011 *

480. 0.0001 I*

510. 0.0001 I*

540. 0.0000 I*

* GAUSIAN BEAM WITH A NARROW LINE WIDTH IS INCIDENI_8 SCANNED *

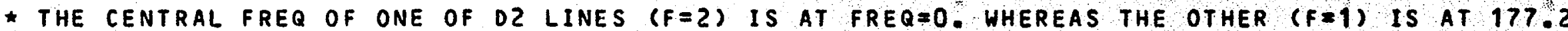

* The WALl EFFEct IS TAKEN INTO ACCOUNT * 
* ANGLE OF INCIDENCE $=15$. DDEGREE * *

<FREQ>

$(* 10 M H Z)$

<R-E-FLECTIIVI-T-Y>

$-2.70 . \quad 0.0700 \mathrm{I}$

$-240.0 .06961$

$-210 . \quad 0.06911$

$-180.0 .0685 \mathrm{I}$

-15.0 .0 .06801 *

$-1.20 .0 .067 .8 .1$

$-90.0 .0681 \mathrm{I}$

$-60.0 .06901$

$-30 . \quad 0.0707 I$

.0. 0.07301

30. 0.07461

60. 0.07481

90. 0.0 .7431

120. 0.0741 I

150. 0.074 .7 .1

180. 0.07571

210. 0.07681

24.0. 0.0.0.7. 0.1

$270 . \quad 0.07701$

300. $0.0 .767 \mathrm{I}$

330. 0.0.0.62 I

360. $0.0757 \mathrm{I}$

390. 0.07521

4.20. 0.07491

450.0 .07461

480. 0.07441

$510 \cdot 0.07421$

540. 0.0741 I

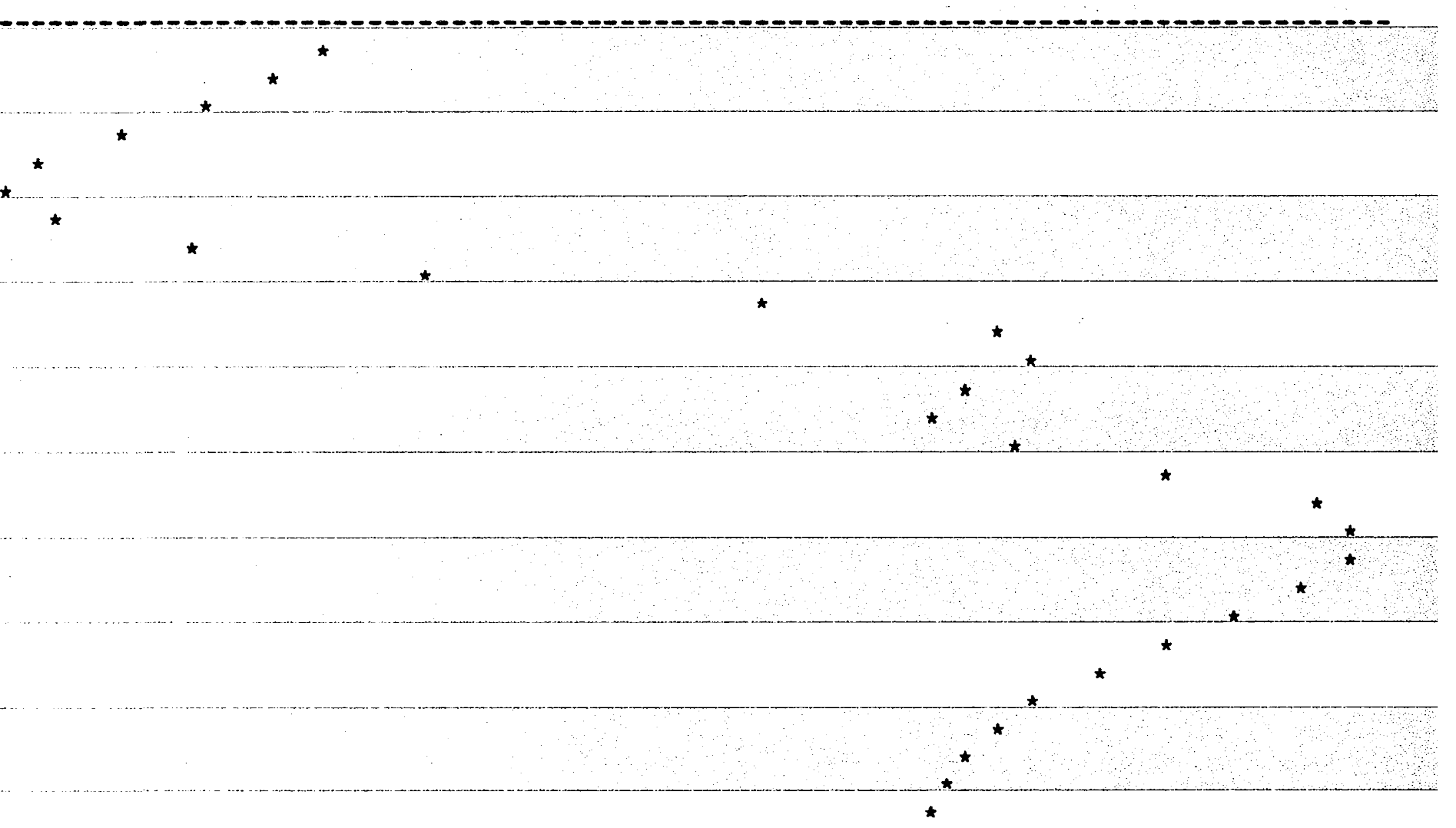

* GAUSIAN BEAM WITH A NARROW LINE WID.TH IS INCIDENT \& SCANNED *

- THE CENTRAL FREQ OF ONE OF D2 LINES (F=2) IS AT FREQ=0. WHEREAS THE OTHER (F=1) IS AT 177.2 * P

* the wall effect is taken into account * 


\section{$<\star \star *$ EXPERIMENTAL CONDITIONS $\star \star \star>$}

<* WALL EFFECTR A.RE TA.KEN INTO ACCOUNT * S

GAUSIAN BEAM WITH A NARROW LINE WIDTH IS INCIDENT \& SCANNED

POLARIZATION IS PERPENDICURAR

RESONANCE WAVE LENGTH OF NA D2 LINE

$$
=0.589 E-04 \text { (CM) }
$$

SPILITTING OF HYPERFINE DOUBLET AT GRAND STATE

$$
=1772 \cdot(+10 M H Z)
$$

DENSITY OF VAPOR $=0.105 E 16$ (PER CUBIC (M)

VAPOR PRESSURE $=0.067$ (MMHG)

TEMPERATURE OF GAS = 619.

HALF OF I/E POINTS ' WIDTH OF INCIDENT LIGHT

$$
=24.0 \quad(* 10 \mathrm{MHZ})
$$

I/E POINTS' DOPPLER WIDTH

$$
=227.2(* 10 \mathrm{MHZ})
$$

HALF OF HALF WIDTH OF PRESSURE BROADENED LINE 1

\begin{tabular}{|c|c|c|c|c|c|c|}
\hline 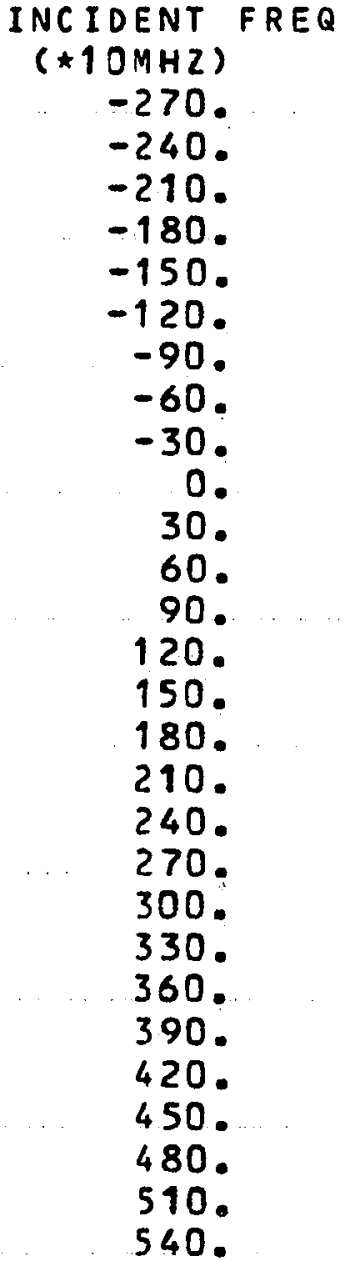 & $\begin{array}{l}\text { DIELECTRI } \\
\text { REAL } \\
1.0169 \\
1.0195 \\
1.0229 \\
1.0265 \\
1.0290 \\
1.0287 \\
1.0250 \\
1.0181 \\
1.0096 \\
1.0013 \\
0.9950 \\
0.9913 \\
0.9 .892 \\
0.9877 \\
0.9857 \\
0.9833 \\
0.9811 \\
0.9794 \\
0.9780 \\
0.9770 \\
0.9776 \\
0.9793 \\
0.9819 \\
0.9841 \\
0.9859 \\
0.9872 \\
0.9883 \\
0.9892\end{array}$ & $\begin{array}{l}\text { C CONST } \\
\text { IMAG } \\
0.0002 \\
0.0004 \\
0.0010 \\
0.0023 \\
0.0045 \\
0.0078 \\
0.0115 \\
0.0146 \\
0.0160 \\
0.0158 \\
0.0144 \\
0.0126 \\
0.0112 \\
0.0102 \\
0.0095 \\
0.008 .5 \\
0.0068 \\
0.0049 \\
0.0035 \\
0.0026 \\
0.0016 \\
0.0010 \\
0.0005 \\
0.0002 \\
0.0001 \\
0.0001 \\
0.0001 \\
0.0000\end{array}$ & $\begin{array}{l}\text { REFRACT } \\
\text { INDEX } \\
1.0084 \\
1.0097 \\
1.0114 \\
1.0132 \\
1.0144 \\
1.0144 \\
1.0128 \\
1.0096 \\
1.0055 \\
1.0014 \\
0.9981 \\
0.9961 \\
0.99 .50 \\
0.9941 \\
0.9931 \\
0.9918 \\
0.9907 \\
0.9897 \\
0.9890 \\
0.9884 \\
0.9888 \\
0.9896 \\
0.9909 \\
0.9920 \\
0.9929 \\
0.9936 \\
0.9941 \\
0.9946\end{array}$ & $\begin{array}{l}\text { PENET } \\
D E P T H \\
0.3 E-01 \\
0.1 E-01 \\
0.5 E-02 \\
0.2 E-0.2 \\
0.1 E-02 \\
0.7 E-03 \\
0.5 E-0.3 \\
0.4 E-03 \\
0.3 E-03 \\
0.3 E-0.3 \\
0.4 E-03 \\
0.4 E-03 \\
0.5 E-03 \\
0.5 E-03 \\
0.6 E-03 \\
0.6 E-03 \\
0.8 E-03 \\
0.1 E-02 \\
0.2 E-02 \\
0.2 E-02 \\
0.3 E-02 \\
0.5 E-02 \\
0.1 E-01 \\
0.3 E-01 \\
0.5 E-01 \\
0.7 E-01 \\
0.1 E-00 \\
0.1 E-00\end{array}$ & $\begin{array}{l}\text { REFRCT } \\
\text { ANGLE } \\
5.3 .9 \\
53.8 \\
53.7 \\
5.3 .6 \\
53.5 \\
53.5 \\
53.6 \\
53.8 \\
54.1 \\
5.4 .5 \\
54.7 \\
54.9 \\
55.0 \\
55.1 \\
55.2 \\
5.5 .3 \\
55.4 \\
55.4 \\
55.5 \\
55.5 \\
55.5 \\
55.4 \\
55.3 \\
55.2 \\
55.2 \\
55.1 \\
55.1 \\
55.0 \\
6 \\
5 \\
5\end{array}$ & $\begin{array}{l}0.1663 \\
0.1651 \\
0.1635 \\
0.1616 \\
0.1602 \\
0.1602 \\
0.1620 \\
0.1653 \\
0.1697 \\
0.1741 \\
0.1774 \\
0.1793 \\
0.1802 \\
0.1810 \\
0.1820 \\
0.1835 \\
0.1848 \\
0.1857 \\
0.186 .6 \\
0.1872 \\
0.1870 \\
0.1858 \\
0.1843 \\
0.1830 \\
0.1821 \\
0.1814 \\
0.1808 \\
0.1803 \\
\end{array}$ \\
\hline
\end{tabular}

$=4.3 \quad(* 1 . \mathrm{MHZ})$

HALF OF HALF WIDTH OF PRESSURE BROADENED LINE 2

$=2.9 \quad(\star 10 \mathrm{MHZ})$ 

* ANGLE OF INCIDENCE =30.0DEGREE *

CFR.EQ>

$(\star 10 \mathrm{MHZ})$

LREFLECTIVITYS

$-270 . \quad 0.1663 I$

-240.0 .1651 I

$-210 . \quad 0.1635$

$-180.0 .16161$

-150. 0.1602 I*

-120 . 0.1602 I*

-90.0 .1620 I

$-60 . \quad 0.16531$

$-30 . \quad 0.16971$

$0.0 .1741 \mathrm{I}$

30. 0.17741

60.0 .17931

90. 0.18021

120. $0.1810 \mathrm{I}$

1.50. 0.18201

180. $0.1835 \mathrm{I}$

210. $0.1848 \mathrm{I}$

24.0.... 0.18.5. I

270. 0.1866 I

300. 0.1872 I

330. $0.1870 \mathrm{I}$

360. 0.18581

390. 0.18431

420. 0.18301

450. $0.1821 \mathrm{I}$

480. 0.1814 I

$510 \ldots 0.1808$ I

540. 0.1803 I

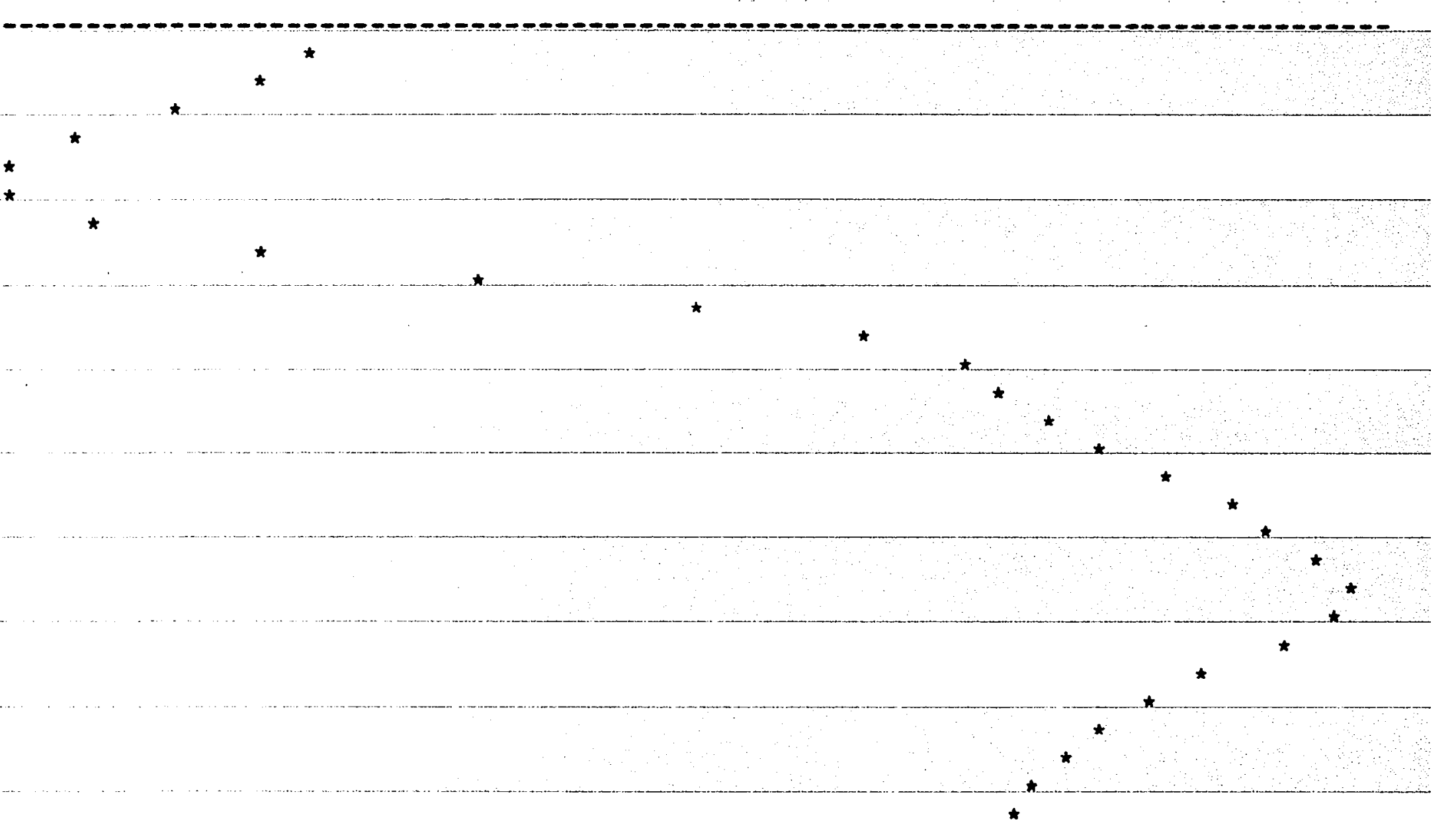

* GAUSIAN BEAM WITH A NARROW LINE WIDTH IS INCIDENT \& SCANNED_*

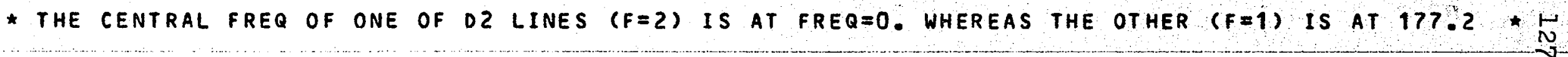

* the wall effect is taken into account * 


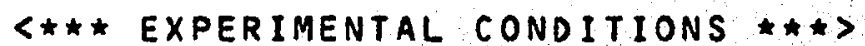

<* WALL EFFECTS ARE TAKEN INTOACCOUNT $*$

GAUSIAN BEAM WITH A NARROW LINE WIDTH IS INCIDENT \& SCANNED

POLARIZATION IS PERPENDICURAR

RESONANCE WAVE LENGTH OF NA DZ LINE

$$
=0.589 \mathrm{E}-04 \quad \text { (CM) }
$$

SPILITTING OF HYPERFINE DOUBLET AT GRAND STATE

$\begin{aligned} \text { DENSITY OF VAPOR } & =177.2 \quad(* 10 M H Z) \\ & 0.105 E 16 \text { (PER CUBIC CM) }\end{aligned}$

VAPOR PRESSURE $=0.067$ (MMHG)

TEMPERATURE OF GAS $=6.19$

HALF OF 1/E POINTS' WIDTH OF INCIDENT LIGHT

$$
=24.0 \quad(* 10 \mathrm{MHZ})
$$

$1 / E$ POINTS DOPPLER WIDTH

$$
=227.2(\star 10 M H Z)
$$

HALF OF HALF WIDTH OF PRESSURE BROADENED LINE 1

$$
=4.3 \quad(* 10 \mathrm{MHZ})
$$

HALF OF HALF WIDTH OF PRESSURE BROADENED LINE 2

$=2.9 \quad(* 10 \mathrm{MHZ})$

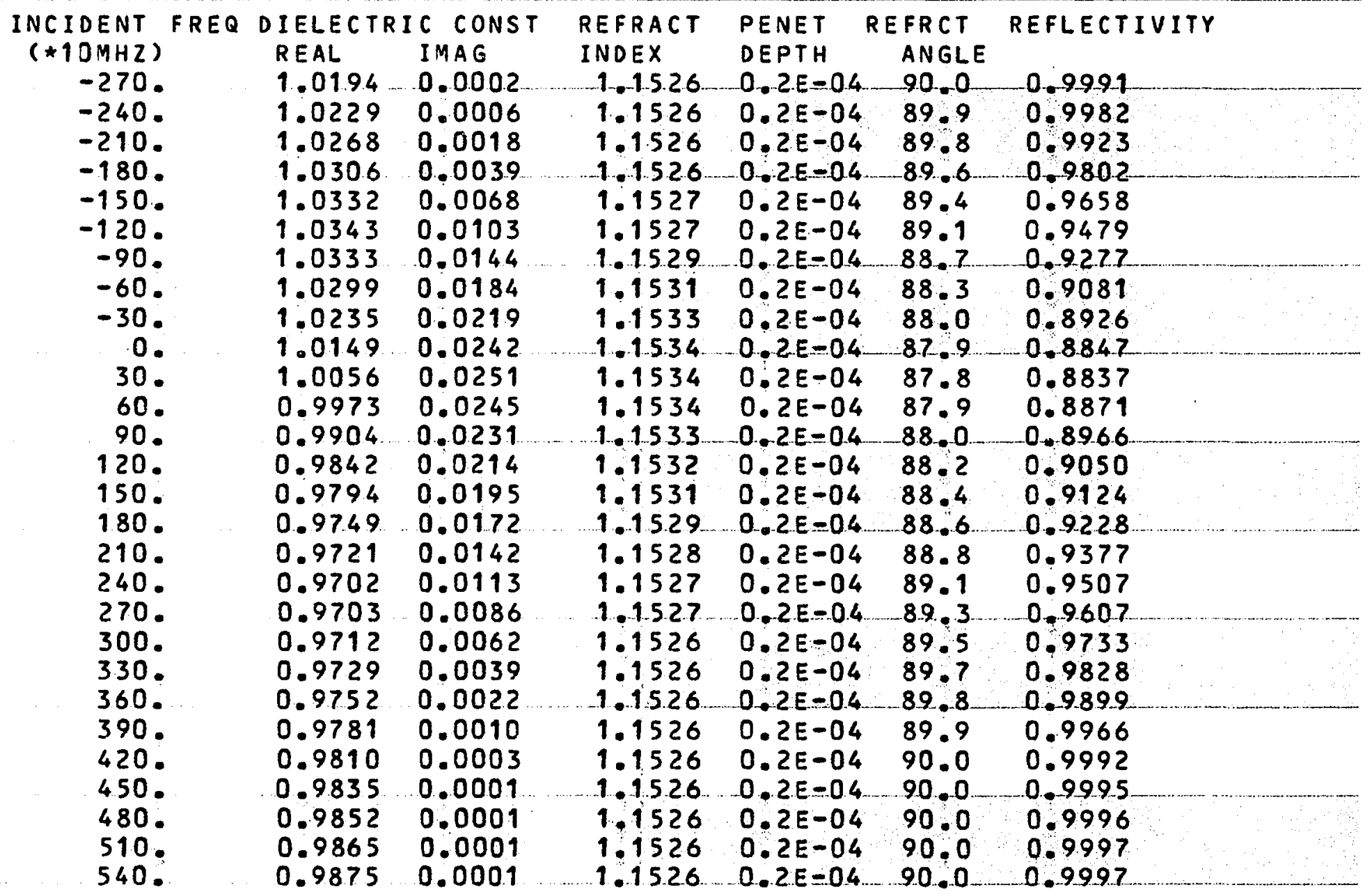



* ANGLE OF INCIDENCE $=45.00 E G R E E *$

〈FREQ>

$(\star 10 M H Z)$

<IMAG PART OF THE DIELECTRICCONSTS

$-270.0 .0002 I$

$-240.0 .00061 *$

$-210 . \quad 0.00181$

$-180.0 .0039 \mathrm{I}$

$-150.0 .0068 \mathrm{I}$

$-120.0 .0 .103 .1$

$-90 . \quad 0.0144 I$

$-60.0 .01841$

$-30.0 .02191$

0.0 .02421

30. 0.02511

60.0 .0245 .1

90. 0.02311

120. 0.02141

150. 0.019 .5

180. 0.0172 I

210. 0.01421

$240 . \quad 0.01131$

270. 0.0086 I

300. $0.0062 \mathrm{I}$

330. 0.00391

360. 0.0022

390. 0.00101

420. 0.0003. 1 *

4.50. 0.0001 I*

480. 0.00011 *

5.1.0....0.0.001 1*

540. 0.0001 I*

* GAUSIAN BEAM WITH A NARROW LINE WIDIH IS INCIDENT \& SCANNED *

* THE CENTRAL FREQ OF ONE OF D2 LINES (F=2) IS AT FREQ=0. WHEREAS THE OTHER (F=1) IS AT 177.2 \& $\stackrel{\text { IS }}{ }$ * the wall effect is taken into account * 
$\star \star A N G L E$ OF INCIDENCE $=45.00 E G R E$ *

$\langle F R E Q\rangle$

<REFLECTIVITYS

$(\star 10 M H Z)$

$-270 . \quad 0.9991$

-240.0 .9982 I

$-21.0 . \cdots 0.99231$

$-180.0 .98021$

$-150.0 .9658 I$

$-120 . \quad 0.94791$

$-90 . \quad 0.92771$

$-60.0 .90811$

$-30 . \quad 0.89 .26$

0.0 .88471 ॠ

30. $0.8837 I \star$

60. $0.88711 *$

90. 0.8 .9661

120. 0.90501

150. $0.9124 \mathrm{I}$

180. $0.9228 \mathrm{I}$

210. 0.9377 I

240.... 0.9.507 I

2.70. 0.9607

300. 0.97331

330. 0.98281

360. 0.9899 I

390. 0.99661

4.20. $0.99 .92 \mathrm{I}$

450. 0.9995 I

480. 0.9996 I

510. 0.9997 I

540. 0.9997 I

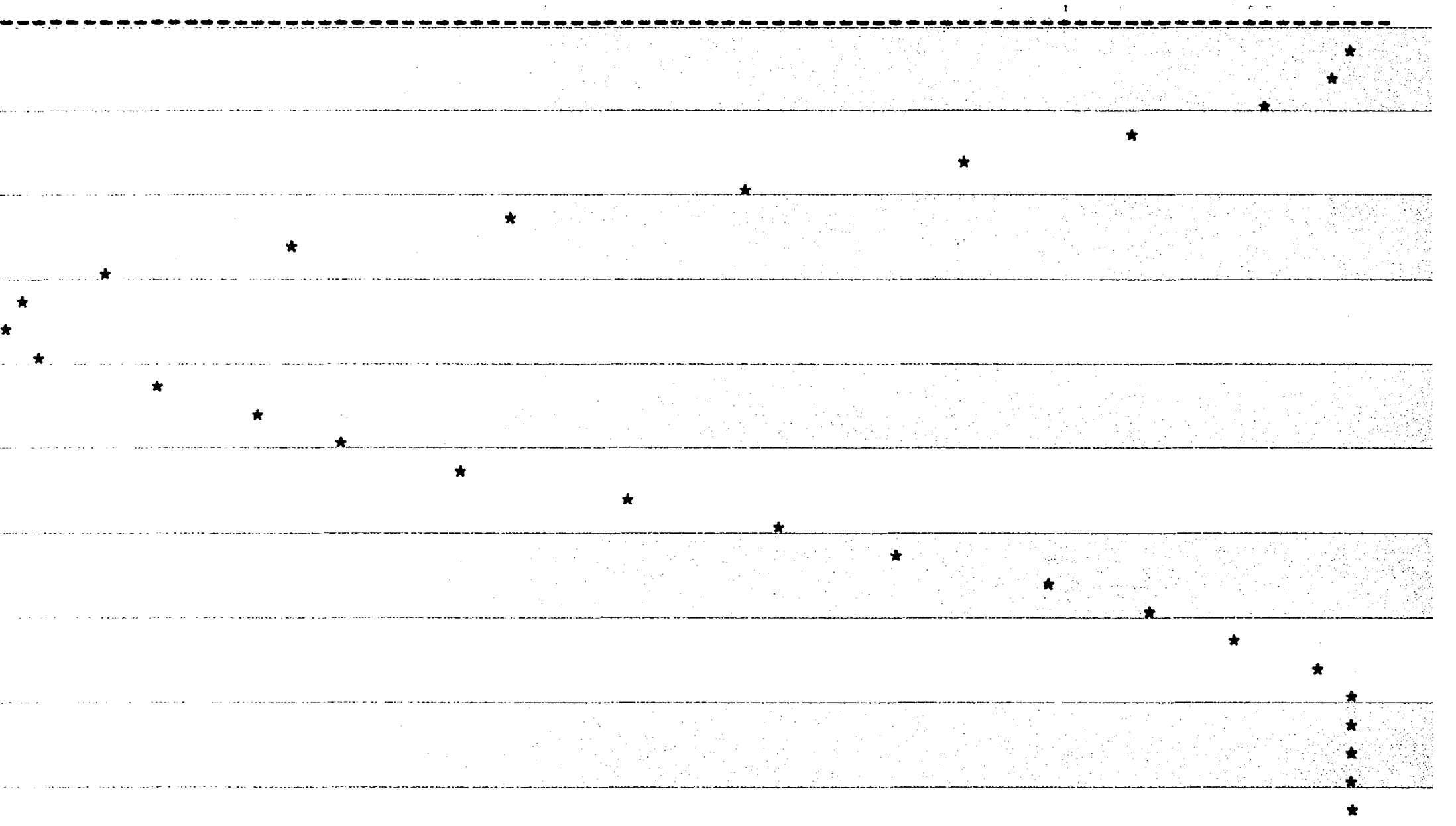

* GAUSIAN BEAM WITH A NARROW LINE WIDTH IS INCIDENT \& SCANNED *

- THE CENTRAL FREQ OF ONE OF D2 LINES (F=2) IS AT FREQ=0. WHEREAS THE OTHER (F=1) IS AT 177.2 *

* the wall effect is taken InTO aCCOUNT * 
* ANGLE OF INC IDENCE $=10.0$ DEGREE * $*$

SFREQ>

SREFLECTIVITYS

$(\star 10 M H Z)$

$-270 . \quad 0.0614$

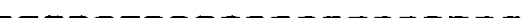

$-240,0.0612$

$-210 . \quad 0.06071$

$-180.0 .0601$

$-150,0.0597$

-120. 0.0595 I*

-90.0 .05971 *

-60.0 .0604 I

-30. $\quad 0.0617$ I

0.0 .06381

30. 0.06601

60. 0.0660 I

90. 0.06501

1.20. 0.06461

150. 0.06511

180. 0.0662

210. 0.0674 I

240. 0.06771

270. 0.0676 I

300. $\quad 0.0673$ I

330. 0.06671

360. 0.06621

390. 0.0658 I

420. 0.06551

450. $0.0653 \mathrm{I}$

480.0 .0651 I

510. $0.0650 \mathrm{I}$

$540.0 .0649 \mathrm{I}$

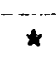

$\star$

$\star$

$\star$

$\star$

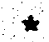

* WHITE LIGHT IS INCIDENT *

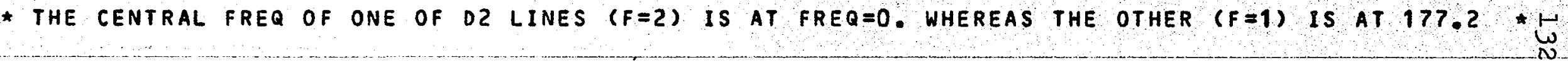

* THE WALL EFFECT IS TAKEN INTO ACCOUNT * 
$\star$ ANGLE OF INCIDENCE $=30.00 E G R E$ *

SFREQS.

$(\star 10 M H Z)$

SREFLECTIVITY

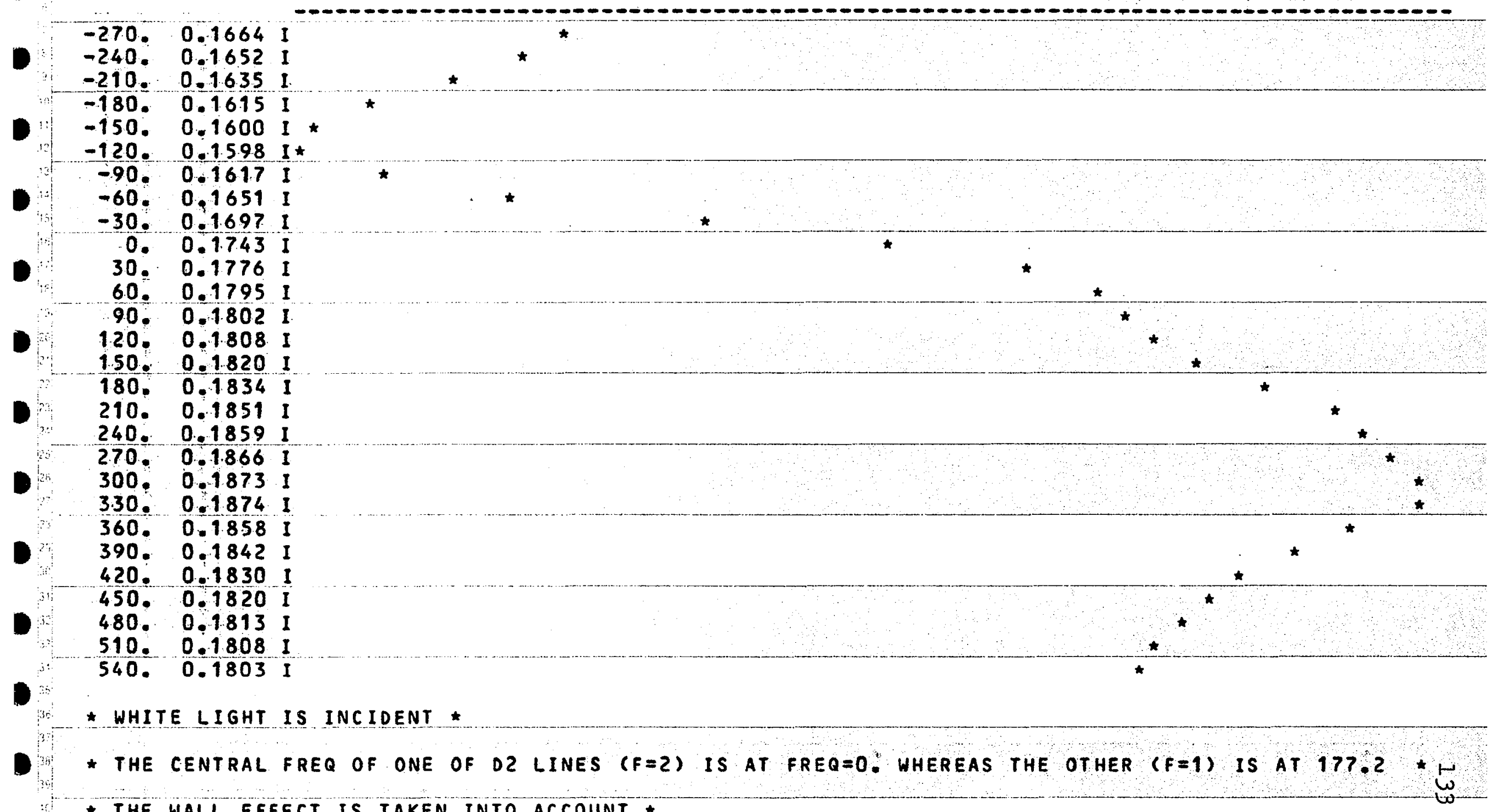

* The WALl effect IS TAKEN INTO ACCOUNT * 
\$\$JOUT, MONI, T

\$ IDENT:RFHYAO01, SATSFECT

D!OFTION:FOFITFAN

\$FORTTIAN

C CALCULATION OF A LINE SHAFE OF A WEAK FROBING BEAM REFLECTEI

C. FROM A SOLID-NA GAS INTEFFACE

C THE STRONG HEAM IS TUNER TO ONE OF NA I2 LINES (F=2, F=J+I,

C. $J=1 / 2$ \& $I=3 / 2)$, WHEN THE WEAK BEAM IS WHITE

C

C SIMFSON'S METHON IS USEI IN NUMERICAL INTEGRATIONG

C

C THE STRONG AND THE WEAK BEAM ARE ALSO SWEEPED ACROSS THE

C RESONANCE LINE WHEN THE WEAK HEAM IS ALSO NARFDW

C

C

C IHOLE $=0$, CALCULATION FOF WITUOT THE STFONG BEAM

C IHOLE =1, CALCULATION FOF THE HOLE STFACTUFE

C ICONTL SEE SUBROUTINE SUSCEF

C FOR IFFOGM $=0$, THE FRORING EEAM IS NARROW

C FOR IFFOGM $=1$, THE FROKING BEAM IS WHITE

C FOR IFROGM $=2,3,4$ SEE SURFDUTINE GRAFH

C

$\mathrm{C}$

*CONSTANTS*

REWAVE = RESONANCE WAVE LENGTH OF NA II2 LINE

GASIIEN = DENSITY DF GAS UAFOR (FER CUAIC CM)

MOLWEI = MOLCULAF WEIGHT OF ATOM

HWI $=$ HALF OF $1 / E$ FOINTS WIDTH OF INCIIENT LIGHT ( 1 OMHZ)

HWN1 = HALF OF HALF WIITH OF FESONANCE LINE( $\$ 1$ OMHZ)

SORIND = FEFFACTIVE INIIEX DF GLASS

DS1 = DSCIRATOF STLENGTH 1

TEMPER = GASTEMFERATURE

XLIGHT = VELOCITY OF LIGHT

FLANK = FLANK CONSTANT

ELMASS = ELECTOFON MASS

GASCON $=$ GAS CONSTANT

XLOSHM = LOSHMIIT'S NUMBER

AANGLE = ANGLE OF INCIIDENCE (DEGREE)

ANGINC = ANGLE OF INCIDECE (FAIIIAN)

EFIIOF $=1 /$ F FOINTS WIITH OF IIOFFLEF SHIFTEII RESONANCE FLER

FAI $=$ FAI

UWGRI $3=A$ CONSTANT IN THE FOTENTIAL ENEFGY OF THE FORM, C/Z* $* 3$

IT IS EXPRESED IN THE IEAYE UNIT "I**2"

WHEFE II**2 IS 1.E-36 ERG*CM**3

IFOWEF =THE FOWER FACTOF OF THE NORMAL IIISTANCE FROM THE SURFACE IN THE FOTENTIAL

SHIFAC $=A$ CONSTANT AFFEAFING IN THE FOTENTIAL ENEFGY EXFFESSION "SHIFAC*C/Z**3" FOR THE EXCITEI ATOM

UDISMI =A DISTANCE (FROM THE GURFACE) WHERE THE FOTENTIAL IIIF IS MINIMUM

YFRELD =FRER SHIFT OF NATUFAL RESONANCE LINE CORFESFONIING TO THE MINIMUM OF THE FOTENTIAL IIIF

ZHOLE =LOCATION OF THE HOLE(SATUFATEI ATOMS) 
IIMENSION $Z(58)$, FEINI (58), IIERE (58), IIECM (58), FEF (58),

8 ANGREN(58), DEFTHN (58),REFLOG (58)

GASREN $=1$. O5E+15

HWI $=6$.

YFREQS $=20.0$

TEMFER $=619$.

FOLARI $=+1$

IFROGM $=0$

ICONTL $=1$

REWAVE $=5.890 E-5$

$X M O L W E=22.9898$

SATFAC $=0.6$

VWGRDZ $=4$.

IFOWER $=3$

FX $=1$, /FLOAT (IFOWEFI)

$X E 24=1, E+8$

SOFIND $=1+63$

$051=0.42$

SHIFAC $=2,4$

UNISMI $=5$.

XLIGHT $=2+99793 \mathrm{E}+10$

F'LANK $=1.05459 E-27$

ELMASS $=9+10953 E-28$

GASCON $=8+31441 E+7$

BOL TZC $=1$. 38042E-16

UWGX $=1 \cdot E-36$

VWGRAX = UWGFII 3 *WWGX

UWFF'SH=SHIFAC $*$ VWGFAX/FLANK

ECXX $X$ FLLANK/BOLTZC/TEMFEF'

$\mathrm{BCX}=\mathrm{ECXX} / \mathrm{SHIFAC}$

$X E 12 H Z=10 \cdot E+11$

$\mathrm{EC}=\mathrm{BCX} \times X \mathrm{E}_{1} \mathrm{ZHZ}$

$X A N G S T=1+E-24$

FAI $=3+14159$

$X L O S H M=2.68675 E+19$

$\Pi O F=2, * G A S C O N * T E M F E F / X M O L W E$

EFCNOF $=2+* S R R T($ IOF) $/$ FEWAUE

$X M H Z=10+E+6$

$X E M 7=1 \cdot E-7$

HWN $1=0.8142 * X E M 7 * G A S L E N / X M H Z / 2$.

EFIOF =EFCIOF $/ X M H Z$

DISTM $3=$ VWFFSH/XANGST/XMHZ/YFREQS

IIISSHI =CEFT (IISTMZ)

YFRELO $=-$ UWFFSH/XANGST/XE12HZ/UIISMI**3

UWC =YFFEQS $* B C$

VWCONS=EXP( WWC)

ULOW $=2$, *HWN1

VHIG $=200$.

VWI I $=$ UHIG-ULOW

FNUU $=U W I I / 10$.

$N U U=I F I X$ (FNUU)

FNU $=2$. *FLOAT (NUU)

$N U=I F I X$ (FNV)

$H U=U W I I / F N U$ 
VALFX3=VWFFSH*XE24/XMHZ

C TO ESTIMATE THE ENEFGY FLUXSWATT/CM**2) OF THE STRONG BEAM,

C THE COLISION FATE IS ASSUMEI TO EE LOMHZ

ENGFLX $=$ SATFAC $* 137.036 * 2 * * X L I G H T * E L M A S S * 2$ *HWN1*XMHZ/FEWAUE/OS1

C

C CONST=CONSTANT AFFERING IN SUSCEFTIEILITY

C

CONST1 =FLANKKWGASUEN*FEWAUE

CONST $2=137$.036*ELMASS*SRFT (FAT) *4, *EFCIOF*FAI**2

CONST $=$ CONST $1 /$ CONST2

WFITE $(6,899)$ CONST

IFOLA=IFIX (FOLARI)

899 FORMAT 1 1H1, $/ / / 3 X$ "THIS IS A CONSTANT AFPERING IN",

\& SUSCEFTIEILITY" $/ 23 X, E 12,5$ )

C

C. VAFOR=VAFOF FFESSUFE IN UNIT OF MMHG

C

UAFOK $=760, *$ TEMFEF $* G A S H E N / X L O S H M / 273+2$

$N A=8$

FNA $=F L D A T(N A)$

$H A=3.2 * H W I / F N A$

CALL FOWER (AVEFLX, ENGFL $X, N A, H A, H W I$ )

AANGLE $=50$.

ANGINC=AANGLE*FAT 180

SORSIN=SORININ *SIN ( ANG INC)

CN=CONST/SOFSIN

IIOPS=EF IOF*SORSIN

nLOW $=-0.8 *$ nOF'S

IHIG $=0.8 *$ IOFS

EFHWI $=1+6 * H W I$

FNRI $=$ IHIG $/ 1$.

$N I n=I F I X$ (FNIII)

FNII $=2$ + *FLOAT (NIIII)

$N D=I F I X$ (FND)

$H n=2$ * *IHIG/FND

FFESHI =-YFFEQS

ZHOLE $=0$.

C A STFONG \& A WEAK BEAM WITH A NAFFOW LINE WIITH ARE SIMULTANEOUSLY

C SCANNEN DUEF THE F=2 LINE SO THAT THE IIETECTEII HOLE IS ALWAYS

C AT THE CENTRAL FREQ OF THE UNSHIFTEI NATURAL RESONANCE LINE

c

C THE WALL-GAS INTERACTIONS ARE NOT TAKEN INTO ACCOUNT

IFRER $=30$

$F S C=2$.

RATIO=FLOAT (IFFEQ) /FSC+O. 5

RATIO2 $=2$ + *FATIO

$I C=I F I X$ (FATIO2)

no $200 \quad I Z=1 * I C$

$I I Z=I Z-1$

FIFREQ $=-F L O A T$ (IFREQ )

$Z(I Z)=F I F F E Q+F L O A T(I I Z) * F S C$

$S L O W=Z(I Z)-E F H W I$ 
C. A CONUOLUTION INTEGRATION W.F. $T$ - A NARFOW WEAK BEAM STARTS

$S B=0$.

$5 B G=0$.

SSUSF $=0$.

SSUSC $=0$

IIO $600 \quad \sqrt{ } \mathrm{B}=1, \mathrm{NA}+1,2$

$\mathrm{JEB}=\mathrm{JE}-1$

HB=HAKFLOAT (JBE)

$X B=S L O W+H E$

$S M R=0$.

SMEG $=0$.

SMSUSF $=0$.

SMSUSC $=0$.

I10 $650 \quad \angle L E=1,2$

$L B=L L E-1$

$F L=F L O A T(L E)$

$X A=X B+H A * F L$

CALL GAUSIA (AIGAUS, $Z$ (IZ), XA, HWI, FAI)

GAUS=AIGAUS

IHOLE $=0$

CALL. SUSCER O FESUM, CMSUM, XA, NI, HII,

\& ILOW, CN,OS1, HWN1, DOFS,

\& ICONTL, IHOLE, SATFAC, FFESHI, IFFOGM, Z(IZ), HWI)

FESUMI =FESUM

CMSUMD=CMSUM

IHOLE $=1$

CALL SUSCEF ( FESUM, CMSUM, XA, NII, HII,

\& IILOW, CN, OS1, HWN1, IIOFS,

\& ICONTL, IHOLE, SATFAC, FFESHI, IFFOGM, $Z$ (IZ), HWI)

RESUMH =RESUM

CMSUMH=CMSUM

FESUM $1=$ RESUMI-FESUMH

CMSUM $1=$ CMSUMII-CMSUMH

CALL FEF INI \& FEINIIX, REFFEX, FEFCMX, ALFAX, BETAX, FESUMI, CMSUMI,

\& SOFINI, ANGINC, F'AI)

REINDI=KEINIX

IIIECM1 $=$ FEFCMX

DIERE $1=$ FEFREX

ALFAN1 =ALFAX

BETAN1 = EETAX

XANGLE $=$ SOFSIN/FEINIII

ANGFEF =ARS IN ( XANGLE)

CALL KEFLEC ( ALFEFLFX, FEFLFX, ALFAN1, BETAN1, ANG INC, ANGREF,

\& POLARI,SORINI)

REFLF $=$ FEFLFX

IF ( JEE , EQ . O) GO TO 602

IF (JBE,EQ , NA)GQ TO 603

IF $(L B+E Q+O) M L B=2$

IF $(L B, E R+1) M L E=4$

60 TO 604

602 IF (LE.EQ.0)MLE=1

$I F(L B, E Q$, I) ML $E=4$

GO TO 604

603 IF $(L E+E Q+O) M L E=1$ 
IF $(L B, E Q, 1) M L E=0$

604 FML $=$ FLOAT (MLE)

IF (JEE + EQ +NA + ANM +LB +EQ + 1) GO TO 605

SMSUSR $=$ SMSUSF+FESUM 1

SMSUSC = SMSUSC+CMSUM1

605 SME $=$ SME+FML *GAUS*REFLF

SMEG = SMEG +FML *GAUS

650 CONTINUE

$S E=5 E+S M E$

$S R G=S B G+S M E G$

SSUSF $=$ SGUSFi+ SMSUSF

SSUSC $=$ SSUSC+SMSUSC

600 CONTINUE

SUMR $=H A * S B / 3$.

SUMEG $=H A * S B G / 3$.

FEF $(I Z)=S U M B / S U M B G$

ARESUM $=$ SSUSF $/$ (FNA+1*)

ACMSUM $=S S U S C$ ( $F N A+1$.

C CALCULATION OF THE AUEFAGE FEFFACTIUE INREX ANI MIELECTRTC

C CONSTANT

CALL REFINII (FEINDX, FEFFEX, REFCMX, ALFAX, BETAX, AFESUM,

\& ACMSUM, SORINI, ANGINC, FAI)

FEINI $(I Z)=$ FEINIX

$\operatorname{IIECM}(I Z)=R E F C M X$

DIERE $(I Z)=$ FEFFEX

$A L F A N=A L F A X$

BETAN $=$ RETAX

REFTHN $(I Z)=$ REWAUE/BETAN/FAI/2.

ANGLE2 $=S O R S I N / F E I N I(T Z)$

ANGFEN $(I Z)=180$, *AFS IN (ANGLE2)/FAI

200 CONTINUE

IF(INTFAC +EQ , 0)WRITE $(6,903)$ AANGLE

IF (INTRAC + EQ + 1 ) WR ITE $(6,904)$ AANGLE

WFI TE $(6,908)$ VAFOR, TEMFER, HWI, HWN1, ZHOLE,

8 AVEFLX,SATFAC

WRITE $(6,906)$

WRI TE $(6,909)$

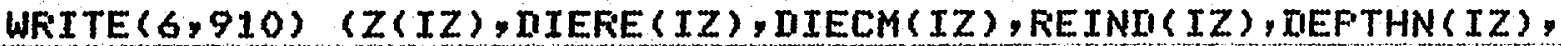

\& ANGFEN (IZ), FEF $(I Z), I Z=1, I C)$

WRITE $(6,955)$

CALL GRAFH(ZHOLE, IFROGM, IC, IFREQ, FSC, DIEFE)

WFITE $(6,956)$

CALL GFAFH (ZHOLE, IFROGM, IC, IFREQ, FSC, DIECM)

WFITE (6,957)

CALL GRAFH(ZHOLE, IFROGM, IC, IFFEQ, FSC, FEINI)

WFIT TE $(6,958)$

CALL GRAFH(ZHOLE, IFKOGM, IC, IFKEQ, FSC, FEF)

C THE PROEING BEAM IS WHITE, WHEN THE STRONG BEAM CFEATES

C A HOLE AT VARTOS INCINENT FRER

C

4100 IUAN $=3$

IPROGM $=1$

ZEFO $=0$.

ID 4600 IUANIE $=1$, IUAN 
INTRAC = IVANIE-3

ZHOLE $=0$

IF (INTRAC +EQ +-2$) Z H O L E=-8$.

IF (INTRAC +EQ, -1 ) ZHOLE $=+16$.

IFREQ $=34$

$F \mathrm{SC}=2$.

FIFFEQ =FLOAT (IFFEQ)

RAT IO=FIFRER $/ F S C+0.5$

FATIO2 $=2$ * *RATIO

$I C=I F I X(R A T I Q 2)$

IFFEQ $=$ IFFEQ - IF IX (ZHOLE)

IF (INTRAC +LE, -1)F IFREQ =F IFFEQ-ZHOLE

I10 $4000 I Z=1, I C$

$I I Z=I Z-1$

SLOW $=$ ZHOLE-EFHWI

$S H I G=Z H O L E+E F H W I$

IF $(I C O N T L+E Q+O) S L O W=Z H O L E-2$, *EFHWI

IF ( ICONTL +EQ , O) SHIG =ZHOLE+2, *EFHWI

$Z(I Z)=-F I F R E(Q+F L D A T(I I Z) * F S C$

$S S L=Z(I Z)-S L O W$

$S S H=Z(I Z)-S H I G$

IHOLE $=0$

CALL SUSCEF (FESUM, CMSUM, Z(IZ), NI, HN,

\& DLOW, CN, OS1, HWN 1, IIOFS, ICONTL, IHOLE,

\& SATFAC, ZHOLE, IFROGM Y Z(IZ), HWI)

RESUMII=RESUM

CMSUMII $=$ CMSUM

IF (SSL , GE + ZERO , ANI - SSH + LE , ZERO) IHOLE $=1$

IF (IHOLE. NE + 1 ) GO TO 4120

CALL SUSCER (RESUM, CMSUM, $Z$ (IZ), NI, HI,

\& DLOW, CN, OS1, HWN1, IOOFS, ICONTL, IHOLE,

\& SATFAC, ZHOLE, IFROGM, Z(IZ), HWI )

RESUMH $=$ RESUM

CMSUMH $=$ CMSUM

FESUM1 =FESUMII-FESUMH

CMSUM1 =CMSUMI-CMSUMH

GO TO 4130

4120 RESUM1 $=$ RESUMD

CMSUM1 = CMSUMTI

4130 CALL REF INI (REINIX, REFREX, REFCMX, ALFAX,

\& BETAX, RESUM1, CMSUM1, SORINI Y ANGINC, FAI)

REIND $(I Z)=$ REINIX

MIERE $(I Z)=$ FEFREX

DIECM $(I Z)=$ REFCMX

$A L F A N=A L F A X$

BETAN=BETAX

DEFTHN $(I Z)=F E W A U E / B E T A N / F A I / 2$.

ANGLE2 $=50 R S I N / F E I N D(I Z)$

ANGREN (IZ) =180. *ARSIN (ANGLE2) /PAI

CALL REFLEC (ALREFLFX, REFLFX, ALFAN, BETAN, ANGINC, ANGFEF,

$\&$ POLALI, SORIND)

$\operatorname{REF}(I Z)=R E F L F X$

4000 CONTINUE

IF (INTRAC \&LE. 0)WFI TE $(6,901)$ AANGLE 
IF ( INTRAC + EQ , 1) WRI TE $(6,902)$ AANGLE

IF (IFOLA+LT+O) WRITE $(6,898)$

IF (IFOLA, GT + O) WRITE $(6,897)$

IF (IFOLA , EQ , O) WRITE $(6,896)$

WRITE $(6,908)$ UAFOF, TEMFER, HWI, HWN1, ZHOLE,

\& AVEFLX, SATFAC

WRITE $(6,909)$

WRITE $(6,910)$ ( $Z(I Z)$, IIERE (IZ), IIECM(IZ),REIND(IZ), IEFTHN(IZ),

\& ANGREN (IZ), FEF (IZ),IZ=1,IC)

WRITE $(6,955)$

CALL GFAFH (ZHOLE, IFFOGM, IC, IFFEQ, FSC, IIEFE)

WRITE $(6,956)$

CALL GRAFH(ZHOLE, IFROGM, IC, IFRER, FSC, IIIECM)

WFITE $(6,957)$

CALL GRAFH (ZHOLE, IFROGM, IC, IFFEQ, FSC, FEINI)

WRITE $(6,958)$

CALL GFAFH (ZHOLE, IFFOGM, IC, IFFER, FSC, FEF)

4600 CONT INUE

$c$ THE SOLIR-GAS INTERACTIONS ARE TAKEN INTO ACCOUNT

C

C A HOLE IS FIFST CFEATEI EY THE STRONG EEAM AT "ZHOLE"

5 : THEN THE INTENSITY IISTFIEUTION OF THE WEAK WHITE

C FFPEING LIGHT IS INUESTIGATEI

\section{IF $=5$}

ZERO $=0$.

TPROGM $=1$

ZHOLE $=-40$.

I0 1600 IFW $=3$, IF

IFOWEF $=I F W-1$

IF (IFOWER , ER + 2 ) UWGRNI $=$ UWGRD $3 /$ VIISMI

IF (IFOWER , ER , 3) UWGRNI =UWGRII

IF ( IFOWER , EQ , 4) UWGKNI=UWGRI 3 *UIISMI

IF (IFOWER , EQ + 2) UALF X = VALFX3/VIISMI

IF ( IFOWER + ER + 3) UALFX $=$ VALLFX 3

IF (IFOWER . ER , 4) UALFX $=$ VALFX3 *UIISMI

$F X=1+/ F L O A T$ ( IFOWER)

UALFAX $=F O W(U A L F X, F X)$

PUX $=P X * U A L F A X$

CALL UWNOFM ( UWCOCF, FX,FUX,NU,HU, VLDW)

IFRE $Q=68$

$F S C=4$.

FIFRER $=$ FLOAT (IFREQ)

RAT IO $=$ FIFREQ $/ F S C+0.5$

RATI02 $=2$. *RATIO

IC $=$ IF IX (FAT IO2)

IFRER $=$ IFREQ - IF IX ( $Z$ HOLE)

FIFREQ $=F$ IFFEQ-ZHOLE

I0 $2000 \quad I Z=1, I C$

$I I Z=I Z-1$

$Z(I Z)=-F I F F E Q+F L D A T(I I Z) * F S C$

IHOLE $=0$

CALL SUSCEV KRESUM, CMSUM,Z (IZ), NI, HI, NU,HU,

\& ILLOW, VLOW, CN,OS1, HWN1, LOFS, IHOLE,

\& SATFAC, ZHOLE, IFROGM , HWI ,F $X, F U X$, WWCOCF) 
RESUMT=RESUM

CMSUMII=CMSUM

IHOLE $=1$

CALL SUSCEV (FESUM, CMSUM, Z(IZ), NI, HII, NU, HU,

8 IILOW, ULOW,CN,OS1, HWN1, IOFS, IHOLE,

\& SATFAC, ZHOLE, IFFOGM, HWI, FX, FUX, UWCOCF,

RESUMH=RESUM

CMSUMH $=$ CMSUM

FESUM1 =RESUMII-FESUMH

CMSUM1 =CMSUMI-CMSUMH

CALL FEFINII (FEINIX, FEFFEX, FEFCMX, ALFAX,

8 BETAX, RESUM1, CMSUM1, SOFINI, ANGINC, FAI)

REINI $(I Z)=$ REINIX

IIERE $(I Z)=$ FEFFEX

IIIECM $(I Z)=$ FEFCMX

$\ldots \quad$ ALFAN=ALFAX

BETAN=BETAX

IIEPTHN $(I Z)=$ REWAUE/BETAN/F'AI/2 +

ANGLE2 =SORSIN/FEINII (IZ)

ANGREN $(I Z)=180$, *ARSIN (ANGLE2)/FAI

CALL FEFLEC (ALFEFLFX, FEFLFX, ALFAN, EETAN, ANGINC, ANGFEF,

$\&$

REF $(I Z)=$ FEFLFX

2000 CONT INUE

WFITE $(6: 902)$ AANGLE

IF (IFOLA,LT, O)WRITE $(6,898)$

IF (IFOLA.GT. O) WFIT TE $(6,897)$

IF (IFOLA,EQ , O) WRITE $(6,896)$

IF ( IFOWER , EQ + 2) WR I TE $(6,832)$ IFOWEF , UWGFiNI

IF (IPOWER + EQ , 3) WR ITE $(6,833)$ IFOWER, VWGFNI

IF (IFOWER , GE , 4 ) WR I TE $(6,834)$ IFOWER , UWGFNI

WRI TE $(6,908)$ VAFOF , TEMFER , HWI , HWN1, ZHOLE,

$\&$ AUEFLX, SATFAC

WRI TE $(6,909)$

WRITE (6,910) (Z(IZ), IIEFE(IZ), IIECM(IZ), REINU(IZ), REFTHN(IZ),

\& ANGKEN (IZ), KEF $(I Z), I Z=1, I C)$

WRITE $(6,955)$

IFROGH $=2$

CALL. GFAFH (ZHOLE, IFFOGM, IC, IFREQ, FSC, IIIEFE)

WRITE $(6,956)$

CALL GRAFH (ZHOLE, IFFOGM, IC, IFFEQ, FSC, IIECM)

WRITE $(6,957)$

CALL GRAPH (ZHOLE, IFROGM, IC, IFRER, FSC, FEINI)

WRITE (6.958)

CALL GRAFH(ZHOLE, IFFOGM, IC, IFREQ, FSC, REF) IFFOGM $=1$

1600 CONT INUE

C

C THE STRONG WEAK NARROW GEAMS ARE SIMULTANEOUSLY SCANNEI

C

$\mathrm{C}$

$\mathrm{C}$

THUS THE INTENSITY NISTRIEUTION OF THE PEAK (IETECTEI EY THE

WEAK EEAM) IS OBSEFUEI

5100 FNAR=IHIG $/ 0.8$ 
ICONTL $=0$

NDN=IFIX (FNDN)

$F N D=2+* F L D A T$ (NDD)

$N I=I F I X$ (FND)

$H \mathrm{H}=2, * \Pi H I G / F N D$

IPROGM $=3$

FREQ 12 $=-Y F R E L O$

$Z E K O=0$.

IHOLE $=0$

CALL SUSCER (RESUM, CMSUM, ZEFO, NI, HD,

\& OLOW, CN, OS1, HWN1, IOFS , ICONTL, IHOLE,

\& SATFAC , ZERO , IFROEM , ZERO, HWI)

RESUMD $=$ RESUM

IHOLE $=1$

CALL SUSCER IRESUM, CMSUM, $Z, N D, H D$,

\& DLOW, CN, OS1, HWN1, IOFS, ICONTL, IHOLE,

\& SATFAC, ZEFO, IFFOGM, ZERO, HWI)

RESUMH =RESUM

RESUM $1=$ RESUMD-RESUMH

FIS $=0.8$

ISHIFT $=2$

I10 3200 IIUW=2, ISHIFT

SHIFAC=FIS*FLOAT (IIUW)

$\mathrm{BCX}=\mathrm{BCXX} / \mathrm{SHIFAC}$

$\mathrm{BC}=\mathrm{BC} \times * \times \mathrm{BE} 12 \mathrm{HZ}$

CMSUM1 $1=1+-0.5 *$ SATFAC $/(S A T F A C+1+)$

CMSUM1 $=$ CMSUM 1 *CN/HWN 1

$F S C=4$.

IFREQ $=$ IFIX (FFEQ12)

RATIO $=$ FREQ12/F SC+0.5

IC $=$ IFIX (RATIO)

no $3100 \quad I Z=1, I C$

$I I Z=I Z-1$

$Z(I Z)=-F R E R 12+F L D A T(I I Z) * F S C$

FREZIZ $=A C * Z(I Z)$

FUNZIZ=EXF (-FREZIZ)

- RESUMZ=FUNZIZ*FESUM1

CMSUMZ =FUNZIZ*CMSUM1

CALL REFINL \& FEINIIX, REFFEX, REFCMX, ALFAX,

\& BETAX Y FESUMZ, CMSUMZ, SOFI INI, ANG INC , F'AI )

FEINM $(I Z)=$ FEINIX

DIEFE $(I Z)=$ FEFFEX

DIECM $(I Z)=$ REFCMX

ALFAN=ALFAX

EETAN= BETAX

DEFTHN $(I Z)=$ FEWAUE $/$ BETAN/PAI $/ 2$.

ANGLE2 $=S O R S I N / F E I N I(I Z)$

ANGREN(IZ) $=180$. *ARSIN (ANGLE2)/FAT

CALL REFLEC (ALFEFLFX, REFLFX, ALFAN, BETAN, ANGINC, ANGREF,

8 FOLAL I, SORINII)

3100

REF $(I Z)=$ REFLFX

CONTINUE

WFITE $(6,902)$ AANGLE

IF (IFOLA + LT, O) WFITE $(6,898)$ 
IF (IPOLA , GT , O) WFI TE (6,897)

IF (IFOLA,ER, 0) WRI ITE $(6,896)$

IF (IFOWEF + EQ + 2) UWGRNI=UWGRI3/UNISMI

IF ( IFOWEF' + ER + 3) UWGRNI =UWGR I3

IF (IFOWER EQ - 4) UWGFNI=UWGFI3*UMISMI

IF (IPOWEF, EQ . 2) WFI TE $(6,832)$ IFOWER , UWGFND

IF (IFOWER , EQ , 3) WRI TE $(6,833)$ IFOWER, UWGFNI

IF (IPOWER , GE , 4) WR I TE $(6,834)$ IFOWER , VWGFNR

WR ITE $(6,1908)$ UAFOF, TEMFEF, HWI, HWN 1 , YFRELO,

\& SHIFAC

WFITE $(6,907)$

WFI TE $(6,1909)$

WRITE (6,910) (Z(IZ), MIERE(IZ), $\operatorname{IIECM(IZ),~REINN(IZ),~DEFTHN(IZ),~}$

\& ANGREN (IZ), KEF (IZ),IZ=1,IC)

WFITE $(6,956)$

CALL GFAFH(ZHOLE, IFROGM, IC, IFFER, FSC, IIECM)

WFITE $(6,958)$

CALL GFAFH (ZHOLE, IFROGM, IC, IFFEQ, FSC, REF)

3200 CONTINUE

C THE INTENSITY IISTRIBUTION OF THE FEAK INTENSITY OF THE

C FEFLECTEI NARROW WEAK EEAM

$\mathrm{C}$

C THE EFFECT OF UAFIOUS FOWEF FACTOFS F=2,3.4 AFE INUESTIGATEI

C FOR THE SAME VALE DF THE FOTENTIAL IIEAFTH CCORRESFONIING TO

C THE MINIMUM OF THE FOTENTIAL IIIF') AT THE IIISTANCE

C $5 * E-8$ CM AWAY FFOM THE WALL SURFACE

$I F=5$

FFER7 $=180$.

HWI $=20$.

$N A=10$

$H A=3,6 * H W I / F L O A T(N A)$

CMSUM11 $=0,5 * 5 A T F A C /($ SATFAC $+1+)$

ZEFIO $=0$.

IIO 5600 IFW=3, IF

IFOWEF $=I F W-1$

IF (IFOWER + ER + 2) UWGFNI=UWGRI3/UIISMI

IF (IPOWER + ER + 3) UWGFNI =UWGRI3

IF (IFOWER + EQ . 4) UWGFNI=UWGRI 3 *UNISMI

IF (IFOWEF + EQ + 2) UALFX =VALFX3/UIISMI

IF ( IFOWEK , ER . 3) VALFX $=$ VALFX 3

IF (IFOWEF + ER + 4) UALFX $=$ VALFX3*UIISMI

$F X=1$, FLOAT (IFOUER )

UALFAX $=F O W($ UALFX $F X$ )

$P U X=F X * U A L F A X$

CALL UWNORM (UWCOCF, FX, FUX, NU, HU, ULOW)

UWCF = UWCOCF

IFFER = IF IX (FREQ7)

$F S C=4$.

RATIO $=$ (FREQ7-2, *HWI ) /FSC+0.5

IC $=$ IFIX (RATID)

II0 $5000 \quad \mathrm{IZ}=1, \mathrm{IC}$

II $Z=I Z-1$

$Z(I Z)=-F R E Q 7+F L O A T(I I Z)$ KFSC

ULOW $1=Z(I Z)-1$. $6 * H W I$ 
AULOW=ABS (ULOW1)

CALL UWNOFM (UWCOCF, FX, FUX,NA,HA,AULOW)

FUNZIZ $Z=$ VWCOCF $/$ UWCF

CMSUM $1=C N *(1 .-F U N Z I Z * C M S U M 11) / H W N 1$

$1130 \mathrm{CALL}$ FEFINIS FEINIX, REFFEX, FEFCMX, ALFAX,

8 BETAX, RESUMI, CMSUM1, SOFINI, ANGINC, FAI)

FETIN $(I Z)=$ FEINAX

IIERE $(I Z)=$ REFREX

IIECM $(I Z)=R E F C M X$

AL.FAN $=A L F A X$

HETAN=EETAX

IIEFTHN $(I Z)=$ REWAUE/BETAN/FAI $/ 2$.

ANGLE2 $=$ SORSIN/FEINI (IZ)

ANGREN (IZ) $=180 . * A R S I N(A N G L E 2) / P A I$

CALL FEFLEC (ALFEFLFX, FEFLFX, ALFAN, EETAN, ANG INC, ANGREF,

\& POLALI , SOFINII)

REFLOG $(I Z)=A L F E F L F X$

REF $(I Z)=$ REFLFX

REFLOG $(I Z)=$ ALREFLFX

5000 CONTINUE

WFITE $(6,902)$ AANGLE

WFITE $(6,960)$

IF (IFOLA,LT,O)WRITE $(6,898)$

IF (TFOLA.GT,O)WRITE $(6,897)$

IF (IFOLA .EQ . O)WFITE $(6,896)$

IF (IFOWER , EQ , 2$)$ WR ITE $(6,832)$ IFOWER , UWGRNM

IF (IFOWEF, EQ , 3) WFI TE $(6,833)$ IFOWEF, VWGFND

IF ( IFOWEF , GE , 4 ) WRI TE $(6,834)$ IFOWER, UWGRNI

WFITE (6,908) UAFOR, TEMFEF, HWI, HWN1, ZHOLE,

$\&$ AUEFLX, SATFAC

WRITE 6,909$)$

WRITE (6,910) (Z(IZ), IIERE (IZ), UIECM(IZ) , REINI (IZ) , IEPTHN $I Z)$,

$\&$ ANGFEN (IZ), FEF (IZ),IZ=1,IC)

WFITE $(6,956)$

IFROGM $=4$

CALL GRAFH(ZHOLE, IFROGM, IC, IFFEQ, FSC, IIECM)

* HRITE $(6,958)$

CALL GRAFH (ZHOLE, IFROGM, IC, IFREQ, FSC, REF)

WFITE $(6,959)$

CALL GRAFH (ZHOLE, IFROGM, IC, IFFER, FSC, REFLOG)

5600 CONTINUE

833 FOFMAT (1HO,5X, ** VAN IEEF WAAL'S FOTENTIAL ENERGY HAS THE*,

$\&$ "FOFM OF THE INUERSE **",/10X,I1, "RII FOWER OF THE NORMAL",

\& "IISTANCE FROM THE SUFFACE", $16 X$, WITH A CONSTANT" ,FS, 1 ,

\& IN UNIT OF E-12*EFG FEF A**3",/)

832 FORHAT 1 1HO, $5 X$ "** VAN IIER WAAL'S FOTENTIAL ENERGY HAS THE" "

\& "FOFM OF THE INUERSE **", $/ 10 X, I 1$, "ND FOWER OF THE NORMAL",

\& NISTANCE FFOM THE SURFACE , $16 \times$, WITH A CONSTANT" ,F5.1,

\& IN UNIT OF E-12*ERG PER A**2",1)

Q34 FORMAT ( $140,5 X$, "** VAN IEE WAAL $S$ FOTENTIAL ENERGY HAS THE",

\& "FORM OF THE INUERSE **" "/1OX,I1, "TH POWEF OF THE NOFMAL",

\& " DISTANCE FFOM THE SURFACE" "/16X,"WITH A CONSTANT" $F 5+1$,

\& IN UNIT DF E-12*ERG FER A**4",/)

898 FORMAT (1HO,23X, "FOLARIZATION IS PARALLEL") 
897 FORMAT (1H0,23X, "POLAFIZATION IS FERFENIICULAR")

896 FORMAT (1HO, 23X, "PLANE POLAFIZED LIGHT AT 45 DEGREE')

909 FORMAT $(1 H 0,17 X, *$ * EXFEFIMENTAL CONLITIONS $>* * /$

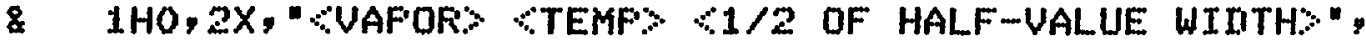

\& "LOC HOLE) CENG FLX STG\% CSATFAC\%" " $3 X$ " "(MMHL)" ,

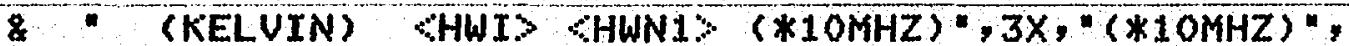

\& $3 X, "$ (WATT/CM**2)" "/1HO,

$8 \quad 3 X, F 5,3,4 X, F 4,0,4 X, F 4,1,3 X, F 4,1$,

\& $11 X, F 6,1,6 X, E 10,3,1 X, F 6,2, /)$

1908 FOFMAT $(1 H 0,17 X, *$ EXFEFIMENTAL CONOITIONS $\% * *$

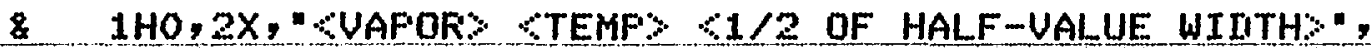

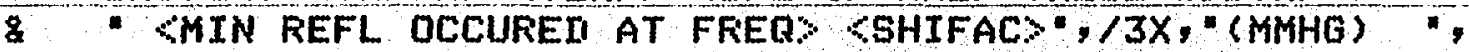

\& (KELUIN) CHWIS SHWN1) ( $\$ 10 M H Z)^{\prime}, 10 X,{ }^{\circ}(* E 12 H Z)^{\circ}, 4 X, / 1 H O$,

\& $\quad 3 X, F 5,3,4 X, F 4,0,4 X, F 4,1,3 X, F 4,1,18 X, F 6,2,14 X, F 3,1,1)$

901 FORMAT 1 1H1, ///3X, **** A WHITE FROBING BEAM INCIIIENT ON",

- A GLASS-NA GAS INTERFACE 称" "/20X" "AT AN" "

\& "INCIIIENT ANGLE OF " "F4,1," IIEGREE",/)

903 FORMAT 1 1H1, $/ / / 3 X$, *** A NARKOW WEAK FROBING EEAM INCIMENT",

* IN A GLASS-NA GAS INTERFACE ***" */20X,

\& "AT AN INCIDENT ANGLE OF " F4,1," IEGREE", $)$

902 FOKMAT 1 1H1,///, "*** THE STRONG \& WEAK BEAMS INCIIIENT ON $A "$ "

\& "THIN METAL LAYER IIEFOSITTEII ON *****/10X*

\& "A GLASS-NA GAS INTERFACE AT AN INCIIENT" "

\& "ANGLE OF " F4,1," IIEGREE" "/)

904 FORMAT $\$ 1 H 1, / / / 3 X$, **** A NARROW WEAK FROBING EEAM INCIMENT",

* ON A THIN METAL LAYER TEFOSIT-" " $110 X$,

\& TEI ON A GLASS-NA GAS AT AN INCIIENT ANGLE OF ",FA.1,

\& IIEGREE* $/ 1)$

906 FOFMAT (1H0,13X, "LINE SHAFE OF THE HOLE IS EXAMINEN", 1 )

907 FOFMAT (1HO,13X, "LINE SHAFE OF THE HOLE ISN'T EXAMINER", $/ 7$

910 FDRMAT $(1 \mathrm{H}, 4 \mathrm{X}, \mathrm{F} 5,0,4 \mathrm{X}, \mathrm{F} 8,6,1 \mathrm{X}, \mathrm{Fg}, 6,1 \mathrm{X}, \mathrm{F} 8,6,1 \mathrm{X}, \mathrm{Eg}, 2,2 \mathrm{X}, \mathrm{F} 4,1$,

\& $\quad 4 X, F B+6)$

909 FOFMAT (IHO, "INCIIIENT FKEQ", 1X, "IIELECTFIC CONST", 2X, "REFFACT",

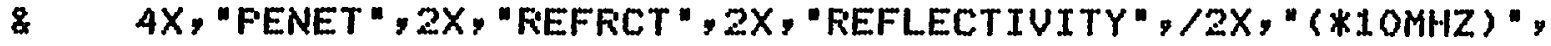

\& 6X, "REAL" "4X, "IMAG", 5X, "INLEX", 6X, "IIEFTH" , 3X, "ANGLE" , /)

955 FOFMAT $(1 H 1, / / / " \angle F R E Q>" 34 X, "<R E A L$ PART OF THE IIELECTFIC",

\& $\quad$ CONST:", /)

1909 FORMAT (1HO, "INCIDENT FREQ", 1X, "IIELECTKIC CONST" ,2X, "REFRACT",

$\& \quad 4 X, " F E N E T " 2 X, "$ REFFCT", 2X, "FEFLECTIUITY", $/ 2 X, "(* E 12 H Z) "$,

\& 6X, "REAL", 4X, "IMAG" , 5X, "INIEX", 6X, "IIEFTH", 3X, "ANGLE",/)

956 FOFMAT $(1 H 1, / / / " \angle F F E Q * 34 X, "$ IMAG FAFT OF THE IIELECTFIC",

\& CONST:,$/ 1$

957 FORMAT $(1 H 1, / / / "$ "FREQP", $34 X$, "CFEFRACTIUE INIEX\%", $)$

958 FORMAT $(1 H 1, / / / " \angle F F E Q \% ", 34 X, " \angle F E F L E C T I U I T Y \% " /)$

959 FOFMAT $(1 H 1, \% / \%$ FREQ\%" $20 X$ " " NATURAL LOG OF THE REFLECTIUITY" \& $>$ ")

960 FOFMAT(1HO, "*** THE IISTRIEUTION OF THE FEAK INTENSITY (IUUE", \& TO THE HOLE) IS EXAMINET ***\%)

9000 STOF

ENII

C. SURKOUTION TO GRAFH IIATA

SUEFOUT INE (GFAFH(ZHOLE, IFROGM, IC, IFFER, FSC, VALUE)

IIIKENGION FLOT (B3), VALUE (IC) 
nATA IIASH/"-"/,RAR/"I" $/, X / " * " /$, ELANK/" "/

no $5 \quad I=1, I C$

IF (I, ER+1) YMAX =UALUE (I)

$I F(I+E R, 1) Y M I N=V A L U E(I)$

$\mathrm{IF}(I, G E, 2)$ GO TO 2

GO TO 5

BIG =VALUE (I) - YMAX

SMALL $=$ UALUE (I ) - YMIN

ZERO $=0$.

IF (BIG, GE, ZERO) YMAX=UALUE(I)

IF (SMALL.LT.ZERO) YMIN=UALUE(I)

5 CONTINUE

DIF $=$ YMAX - YMIN

Lio $10 \quad K=1,83$

FLOT $(K)=$ IIASH

CONT INUE

IF (IFFOGM +EQ + 3)WRITE $(6,21)$ FLOT

IF (IFROGM + NE + 3) WRITE $(6,20)$ FLOT

20 FOFMAT $(1 X, "(* 10 M H Z) ", / 1 H 0,17 X, 83 A 1)$

21 FORMAT $(1 X, "(* E+13 H Z) ", / 1 H 0,17 X, 83 A 1)$

IIO $30 \quad K=1,83$

FLOT $(K K)=E L A N K$

30 CONTINUE

FLOT $(1)=\mathrm{BAF}$

no $60 \quad K Z=1, I C$

$K Z Z=K Z-1$

$Z=-F L O A T(I F R E Q)+F L O A T(K Z Z) * F S C$

YIIIF=UALUE (KZ) - YMIN

$Y N=Y I I F * 80+\angle D I F+2 \cdot 5$

$N Y=I F I X(Y N)$

FLOT $(N Y)=X$

WRITE $(6,40) Z$, VALUE (KZ),FLOT

40 FORMAT $(1 X, F 5,0,2 X, F 8,6,1 X, 83 A 1)$

FLDT (NY) $=$ FLANK

60 CONTINUE

IF (IFROGM +EQ + O) WRITE $(6,50)$

IF ( IFROGM +EQ + 1) WFITE $(6,51)$ ZHOLE, ZHOLE

IF (IFROGM , EQ , 2) WRITE $(6,52)$ ZHOLE

IF (IFROGM, GE, 3 ) WRITE $(6,53)$

50 FORMAT (1HO "* THE NARROW STRONG \& WEAK BEAMS ARE SIMULTANE",

\& OUSLY SCANNEI DUER NA $F=2$ LINE $(F=J+I, J=1 / 2 \& I=3 / 2) *$ *

\& IX, * THE SATURATED ATOMS IIETECTEI BY THE WEAK BEAM ARE*

\& "NEAFI Y AT REST *"/1X** THE PEAK FREQ IS THEREFOFE THAT",

\& OF THE UNSHIFTEI LINE *")

51 FORMAT (1HO, "* THE CENTRAL FFER OF THE STRONG BEAM IS TUNEI",

* "TO "F5+1,"*",/1X,"* THEN THEASE SATURATEI ATOMS AFE" ,

\& " RETECTEI EY THE WEAK WHITE LIGHT RESULT IN THE FEAK AT "

\& F $5.1, * *)$

52 FORMAT (1HO, "THE CENTRAL FREQ DF THE STRONG EEAM IS TUNEI TO ",

\& F5.1, "* " $/ 1 X$, "THEN THEASE SATURATED ATOMS UNMER THE",

\& WALL INTERACTIONS ARE DETECTEI EY WHITE LIGHT *")

53 FORMAT 1 1HO, "* THE NARFOW STRONG \& WEAK BEAMS ARE SIMULTANE",

8 "OUSLY SCANNER DUEF NA $F=2$ LINE $(F=J+I, J=1 / 28 I=3 / 2) * *$

\& 1X, * THE SATURATED ATOMS IIETECTEI ARE NEARLY AT REST **, 
$\& / 1 \times$, *THE IISTRIBUTION OF THE FEAKS (IIUE TO THE HOLES) IS ", \& ELOTTEN *")

RETURN

ENR

C

C SURROUTINE TO CALCULATE AVERAGE FOWER

C SURROUTINE FOUEF (AUEFIX,ENGFL $X, N, H, H W T$ )

SFAA $=0$.

IIO $10 \quad J=1, N+1,2$

$J L=J-1$

$\mathrm{D}=\mathrm{H} * \mathrm{FLOAT}(\mathrm{JL})-1+6 * H W I$

$\mathrm{A} 1=-\mathrm{D} * * 2 / \mathrm{HWI} * * 2$

$\operatorname{EA} 1=\operatorname{EXP}(A 1)$

SRAA $=$ SRAATEAI

10 CONTINUE

AUEFL X=ENGFLX $X S R A A /(F L O A T(N)+1)$

RETURN

ENII

C SUEROUTINE TO CALCULATE SUSCEFTIEILITY FOR THE WEAK PROEING

C BEAM

C SOLIV-GAS INTERACTIONS ARE ABSENT

SUEROUT INE SUSCER (RESUM, CMSUM, Z,N,H,

$\&$ XLOWAL, C, OS1, HWN1, IIOFS, ICONTL,

\& IHOLE, SATFAC, ZHOLE, IFROGM, XINC, HWI )

5 SREAE $=0$.

SCMAE $=0$.

IIO $16 \quad L A=1, N+1,2$

$L L A=L A-1$

DAL $=H * F L O A T(L L A)+X L O W A L$

SRRAE $=0$ :

SCRAB $=0$.

Ii) $17 \quad I I=1,2$

$N N=I I-1$

FNN=FLOAT (NN)

$A L=D A L+H * F N N$

ALL1 $=-4 * *($ ALL*2) $/$ LIOFS**2

EAL $1=\operatorname{EXF}(A L L 1)$

C IF ICONTL=0, A FREQ IIEFENIENCE ON SATFAC IS NOT TAKEN INTO

C ACOUNT

C IF ICONTL $=1$, IT IS TAKEN ACOUNT

IF (ICONTL,EQ. O) GO TO 100

IF (IFROGM,EQ +, , AND, IHOLE,EQ, 1) ALL. $2=((Z-X I N C) * * 2) / H W I * * 2$

IF (IPROGM EQ + 1 ANM . IHOLE +ER + 1$) A L L Z=((Z-Z H O L E) * * 2) / H W I * * 2$

IF (ALL2.GT + 50) GO TO 80

EAL.2=EXP(-ALL2)

SATFA1 $=$ SATFAC $* E A L 2$

GO TO 100

BO SATFA1 $=0$.

100 AL $Z 1=A L-Z$

IF (IFFOGM +EQ + O , ANI , IHOLE,EQ , 1) ALZFI=AL+Z

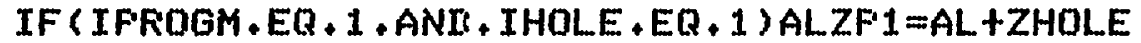

$I F(I F R O G M, E Q$ + 3 ) ALZZF1=AL

IF (IHOLE +EQ + 1) ALZF'2=ALZF' $1 * * 2$ 
IF (IFROGH +ER + 3) ALZF1 $=A L$

$A L Z 2=A L Z 1 * 2 * 2$

HWN2 $=H W N 1 * * 2$

$A L X 1=A L Z 2+H W N 2$

IF $(I C O N T L+E Q . O) S A T F A 1=S A T F A C$

SATFA2 $=1$ + +SATFA1

IF (IHOLE.ER + 1 ) HOLE $=5 A T F A 1 * H W N 2 / 2+/(A L Z F 2+H W N 2 * S A T F A 2)$

RFAL $1=E A L 1 * 0 S 1 * A L Z 1 / A L \times 1$

CFAL $1=E A L 1 * H W N 1 * 0 S 1 / A L X 1$

IF (IHOLE + EQ + 1) FFAL $1=H O L E * F F A L 1$

IF ( IHOLE , EQ 1 ) CFAL 1=HOLE*CFAL 1

IF (LLA+EQ+0)60 TO 12

IF (LLA,EQ,N)GO TO 13

$I F(N N, E Q, O) N C=2$

$I F(N N+E R+1) N C=4$

GO TO 14

$12 I F(N N+E Q, 0) N C=1$

IF $(N N, E Q, 1) N C=4$

GO TO 14

$13 \quad$ IF $(N N, E Q+0) N C=1$

IF $(N N \cdot E Q \cdot 1) N C=0$

$14 \quad F N C=F L O A T$ (NC)

SRFAE = SRFAB+FNC*RFAL 1

SCRAB = SCRAB +FNC*CFALI

17 CONTINUE

SREAR = SRRAR+SREAE

16 CONTINUE

FUNREY $=H * S F E A B / 3$.

FUNCMY $=H$ HSCMAE $/ 3$.

31 KESUM $=$ C*FUNFEY

CMSUM $=$ C*FUNCMY

32 RETUFN

END

C SUEROUTINE FOF THE SUSCEFTIBILITY OF THE WEAK EEAM

C WHERE THE SORID-GAS INTERACTIONS ARE FORM OF $1 / Z * * F$

C

SUBROUTINE SUSCEU RRESUM, CMSUM, Z,N,H,NU,HU,

\& XLOWAL, VLOW, C, OS1, HWN1, IOOPG,

$\&$ IHOLE, SATFAC, ZHOLE, IFFOGM, HWI, FX, FUX, UWCOCF,

$S R R=0$

$S C R=0$.

IF $(I H O L E+E Q+O) N U=0$

no $20 \quad I V=1, N U+1,2$

IF $\Upsilon$ IHOLE, EQ.0)GO TO 1

$I U U=I V-1$

IUU=HU*FLOAT (IUU) + ULOW

$S R R U=0$.

$S C R V=0$.

$L L V=2$

1 IF $T$ IHOLE, EQ . O OLLU $=1$

I0 $15 \quad L V=1, L L U$

IF (IHOLE, EQ + O) GO TO 2

$M M=L V-1$ 
FMM $=$ FLOAT (MM)

$U 1=$ IU+HU*FMM

UFREQ $=1+/{ }_{1}$

$F \times 1=F \times+1$.

UY $=F$ OW (UFREQ,$F \times 1)$

IF (IHOLE +EQ , O) VY $=1$.

SREAE $=0$.

SCMAE $=0$.

no $16 \quad L A=1, N+1,2$

LLA $=L A-1$

IIAL $=H * F L O A T$ (LLA) +XLOWAL

SFRAB $=0$.

SCRAB $=0$,

DO $17 \quad I I=1,2$

$N N=I I-1$

FNN=FLOAT (NN)

$A L=I I A L+H * F N N$

ALL $1=-4$ * * (AL**2)/IOPS**2

$E A L 1=E X P(A L L 1)$

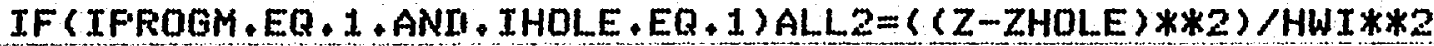

IF (ALL2, $(3 T+50) 60$ TO 80

$E A L 2=E X P(-A L L L)$

SATFA1 $=$ SATFAC*EAL2

60 TO 100

80. SATFA1 $=0$.

100 ALZ1 $=A L$

IF (IHOLE +EQ . 1 ) ALZF $1=A L+Z+U 1$

IF (IHDLE, EQ + 1 ) ALZF2=ALZF $1 * * 2$

ALZZ2=ALZ1 $1 * 22$

$H W N 2=H W N 1 * * 2$

$A L X 1=A L Z 2+H W N 2$

SATFA2 $=1 .+$ SATFA1

IF (IHOLE,EQ + 1 ) HOLE =SATFA1*HWN2/2,/(ALZF2+HWN2*SATFA2)

FFAL $1=U Y * E A L 1 * 0 S 1 * A L Z 1 / A L X 1$

CFAL $1=U Y * E A L 1 * H W N 1 * 051 / A L X 1$

IF ( IHOLE +EQ + 1$)$ FFAL $1=$ HOLE $* F F A L 1$

IF ( IHOLE +EQ + 1) CFAL 1 =HOLE*CFAL 1

IF (LLA,EQ,O)GO TO 12

IF(LLA,EQ,N)GO TO 13

IF $(N N, E Q, O) N C=2$

$I F(N N, E Q, 1) N C=4$

GO TO 14

12 IF $(N N, E Q, O) N C=1$

$I F(N N \cdot E Q, 1) N C=4$

60 TO 14

13 IF $(N N, E Q+0) N C=1$

IF $(N N+E Q+1) N C=0$

$14 \quad F N C=F L O A T$ (NC)

SRRAB =SRRAE+FNC*RFAL1

SCRAB $=$ SCRAB+FNC*CFAL 1

17 CONTINUE

SREAB=SRRAE+SREAE

SCMAE= SCMAE+SCFAE

16 CONTINUE 
FUNREV $=H * S R E A E / 3$.

FUNCMU $=H * S C M A B / 3$.

IF (IHDLE,EQ + O) GO TO 3

IF (IUU,EQ+O)GO TO 22

IF (IVU.ER.NU)GO TO 23

$I F(M M+E R .0) M C=2$

IF $(M M, E Q, 1) M C=4$

GO TO 24

$22 I F(M M+E Q+O) M C=1$

$I F(M M+E R \cdot 1) M C=4$

GO TO 24

23 IF $(M M+E R, 0) M C=1$

$I F(M M+E Q+1) M C=0$

$24 \quad F M C=F L O A T$ ( $H C$ )

SFRU = SREU+FMC *FUNREU

SCRV $=$ SCRU+FMC*FUNCHU

15 CONTINUE

SRR = SRK +SRKV

$S C R=S C R+S C R V$

20 CONTINUE

RESUM $=$ C*FUX*HU*SRF/3 / NWCOCF

CMSUM $=\mathrm{C} * F \cup X * H \cup * S C F / 3, / U W C O C F$

60 TO 4

3 RESUM=C*FUNREU

CMSUM $=$ C*FUNCMU

$4 \quad$ RETUFN

ENII

SUBFOUTINE UWNORM(UWCOCF, FX,FUX,N,H,XALLX)

$S F A A=0$.

no $10 \quad J=1, N+1,2$

$J L=\sqrt{L}-1$

D=H*FLOAT (JL) +XALLX

SRAAA $=0$.

IO $5 \quad L=1: 2$

$M=L-1$

$n M=F L O A T(M)$

$\mathrm{DU}=\mathrm{n}+\mathrm{H} * \mathrm{n} M$

UFREQ $=1, /$ IV

$F \times 1=F \times+1$.

$U Y=F O W(U F R E Q, F \times 1)$

IFSUL,EQ.O)GO TO 2

IF (JL,EQ,N)GO TO 3

$I F(H+E Q+O) M L=2$

$I F(M, E Q, 1) M L=4$

GO TO 4

$2 \quad I F(M+E Q+O) M L=1$

$I F(M \cdot E Q+1) M L=4$

GO TO 4

$3 \quad I F(M, E Q+O) M L=1$

IF (H.EQ 1$) M L=0$

4 IIML $=F L O A T$ (NL)

SRAAA =SFAAA+IIML $* U Y$

5 CONTINUE

SRAA $=$ SRAA $S R A A A$ 
10 CONTINUE

VWCOCF $=$ FUX*H $* S R A A / 3$

RETURN

END

C SUEROUTINE TO CALCULATE REFFACTIUE INIEX

SUBROUT INE REFINI (FEINUX, REFREX , REFCMX, ALFAX, BETAX,

\& SUSREX, SUSCMX, SOINIX, ANGINC, FAI)

REFREX $=1++4$ +*PAI*SUSREX

FEFCHX $=2$ * *FAI $*$ SUSCMX

FART $1=($ SOINDX $* S I N(A N G$ INC $) ~) * * 2$

$A=R E F R E X-F A R T 1$

$B=2$, *REFCMX

C:=REFREX+FART 1

$\mathrm{n}=0$.

$A B=A E S(A)$

IF $(A E-E) 60,61,62$

60 $\quad X=A / T$

$X \mathrm{RT} T=1 \cdot+\mathrm{X} * * 2$

$A L F A=E *(S A R T(X F T)+X) / 2$.

AL.FAX $=$ SQRT (ALFA)

BETA=E* (SQRT $(X F T)-X) / 2$.

$E=S R R T$ (XFT) $-X$

IF (E, EQ, D) BETAX $=0$.

RETAX $=$ SQRT ( $B E T A)$

FARTX $=(C+B * X F T) / 2$.

FEINIX $=$ SRRT (FARTX)

60 TO 63

$61 \quad$ ALFA $=B *(1,+\operatorname{SQRT}(2)) /$,2 .

ALFAX $=S R R T$ ( ALFA)

BETA $=$ B* $(\operatorname{SRRT}(2)-1) /$,2 .

EETAX $=$ SQRT ( $B E T A$ )

FARTE $=(C+B *(1+$ SQFT $(2))) /$,2 .

REINDX $=S$ QRF'T (F'AFITE)

60 TO 63

$62 \quad Y=B / A$

$Y R T=1+Y * * 2$

ALFA=A* $(1+$ +SRRT $(Y R T)) / 2$.

IF $(A, L T, D) A L F A=A B *(S Q F T(Y F T)-1+) / 2$.

AL.FAX $=S R R T$ ( ALFA)

EETA $=A *(S Q R T$ (YFT) $-1+) / 2$.

IF (A.LT, DI) BETA=AE* (SRRT (YRT) $+1,1) / 2$.

$F=S Q R T$ ( YRT $)-1$

IF (F,ER , II) EETAX $=0$.

BETAX $=S$ SRT (EETA)

FAFTY $=(C+A * Y R T) / 2$.

FEINIX $=S Q R T$ ( $P A R T Y$ )

63 IF (REINIX**2-FART 1) $64,65,65$

$64 \quad X C 1=$ REFCMX**2/(FAFT1-REINIX**2)

$X C 2=X C 1+F A R T 1$

$X C=\operatorname{SOFT}(X \mathrm{CO})$

REINIX $=X C$

65

RETUF'N

ENI 
C SURROUTINE TO DEFINE A FUNCTIONAL FORM OF REFLECTIUITY SUEROUT INE REFLEC (ALREFLFX, REFLFX, ALFAX, BETAX, ANGINC, \& ANGREF, FOLARI, SOINIX) $A C=\operatorname{COS}(A N G I N C)$ $A S=S I N(A N G I N C)$

RNUMAX $=($ SOINDX*AC-ALFAX) $* * 2+$ BETAX $* * 2$

RDENO $=($ SOINDX*AC+ALFAX) $* * 2+$ HETAX**2

ALFNUMAX $=A L O G$ (FNUMAX)

ALRIENO $=A L O G$ (RIIENO)

$S A=$ SOINIIX*AS

$X A=(A L F A X * * 2-B E T A X * * 2+5 A * * 2) * A C$

XAN $=X A-S O I N I X * A L F A X$

$X \mathrm{~B}=2$. *ALFAX*BETAX

$X E N=X E-S O I N I X * B E T A X$

RNFER: $=X A N * * 2+X E N * * 2$

$X A D=X A+S O I N I X * A L F A X$

XED $=X B+S O I N D X * E E T A X$

FIIFER $=X A \cap * * 2+X E N * * 2$

ALRNFER $=A L D G$ (RNPER)

AL.RIFER=ALDOG (RDFEF)

IF (FOLARI $)>01,702,703$

701 REFLFX=RNFER/FIFER

ALREFLFX $=A L R N F E F-A L F I F E R$

GO TO 705

703 REFLFX=RNUMAX/FIENO

ALREFLF $X=A L R N U M A X$-ALFIENO

GO TO 705

702 REFLFX $=.5 *$ (FNFER/RIFER+RNUMAX/RIENO)

ALREFLFX $=0.5 *$ (ALFNFER+ALRNUMAX-ALRIFER-ALRDENO)

705 RETURN

ENII

C SURFOUTINE TO FIND THE SRUARE OF THE INCIUENT LIGHT AMFLITUME

SURROUTINE GAUSTA(AIGAUS, XINC, AAZ, HWI, PAI)

$A Z=A A Z-X I N C$

AIG $=(A Z * * 2) / H W I / H W I$

AIGAUS=EXF (-AIG)

RETURN

ENII

\$ EXECUTE

\$LIMIT: $20,37 K,, 6000$

$\$$ :SYSOUT:06, ORG

\$: ENDJJEE 


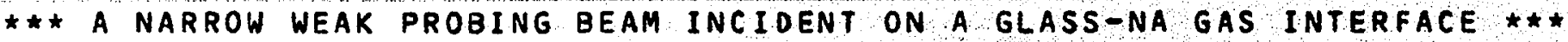
AT AN INCIDENT ANGLE OF 50.0 DEGREE

\section{$\star<$ EXPERIMENTAL CONDITIONS $>*$}

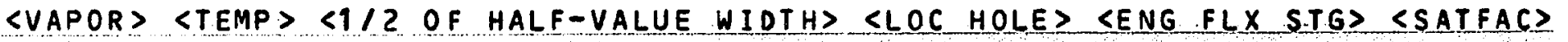
$(M M H G)$ (KELVIN) $\angle H W I\rangle<H W N 1\rangle(\star 10 M H Z)(\star 10 M H Z)$ (WATT/CM* $* 2)$
0.067
619.
$6.0 \quad 4.3$
0.
$0.381 E-02$
0.60

LINE SHAPE OF THE HOLE IS EXAMINED

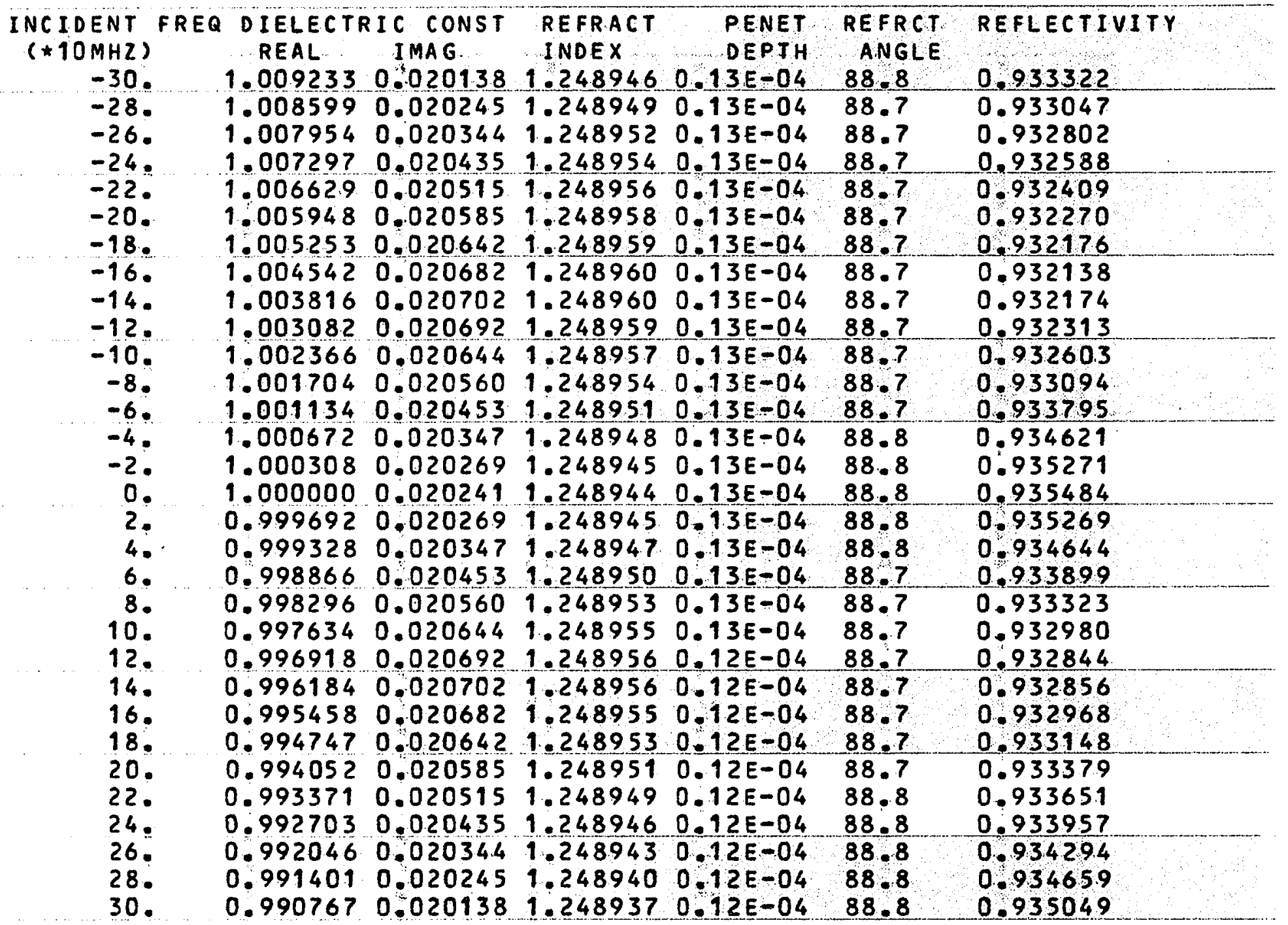


-30 . 0.020138 I* $-28 . \quad 0.020245 I$ -26. 0.0203441 $-24.0 .020435$ -22. 0.0205151 $-20.0 .0205851$ $-18.0 .0206421$ $-16 . \quad 0.0206821$ $-14.0 .0207021$ $-12.0 .020692$ $-10 . \quad 0.020644 I$ $-8.0 .0205601$ $-6.0 .0204531$ $-4.0 .0203471$

-2.0 .020269 I

0. 0.020241 I

2. 0.0202691

4. 0.020347

6. 0.0204531

8. 0.020560 I

10. 0.0206441

12. 0.0206921

14. 0.020702 I

16. 0.020682 I

18. 0.020642 I

20. $0.020585 \mathrm{I}$

22. 0.020515 I

24. 0.020435 I

26. 0.0203441

28. 0.020245

30. $0.020138 \mathrm{I}$ *

$\star$ THE NARROW STRONG \& WEAK BEAMS ARE SIMULTANEOUSLY SCANNED OVER NA F $=2$ LINE $(F=J+1, J=1 / 28 I=3 / 2)$ *

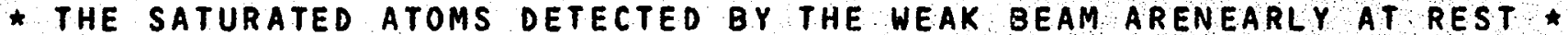

* THE PEAK FREO IS THEREFORE THAT OF THE UNSHIFTED LINE *

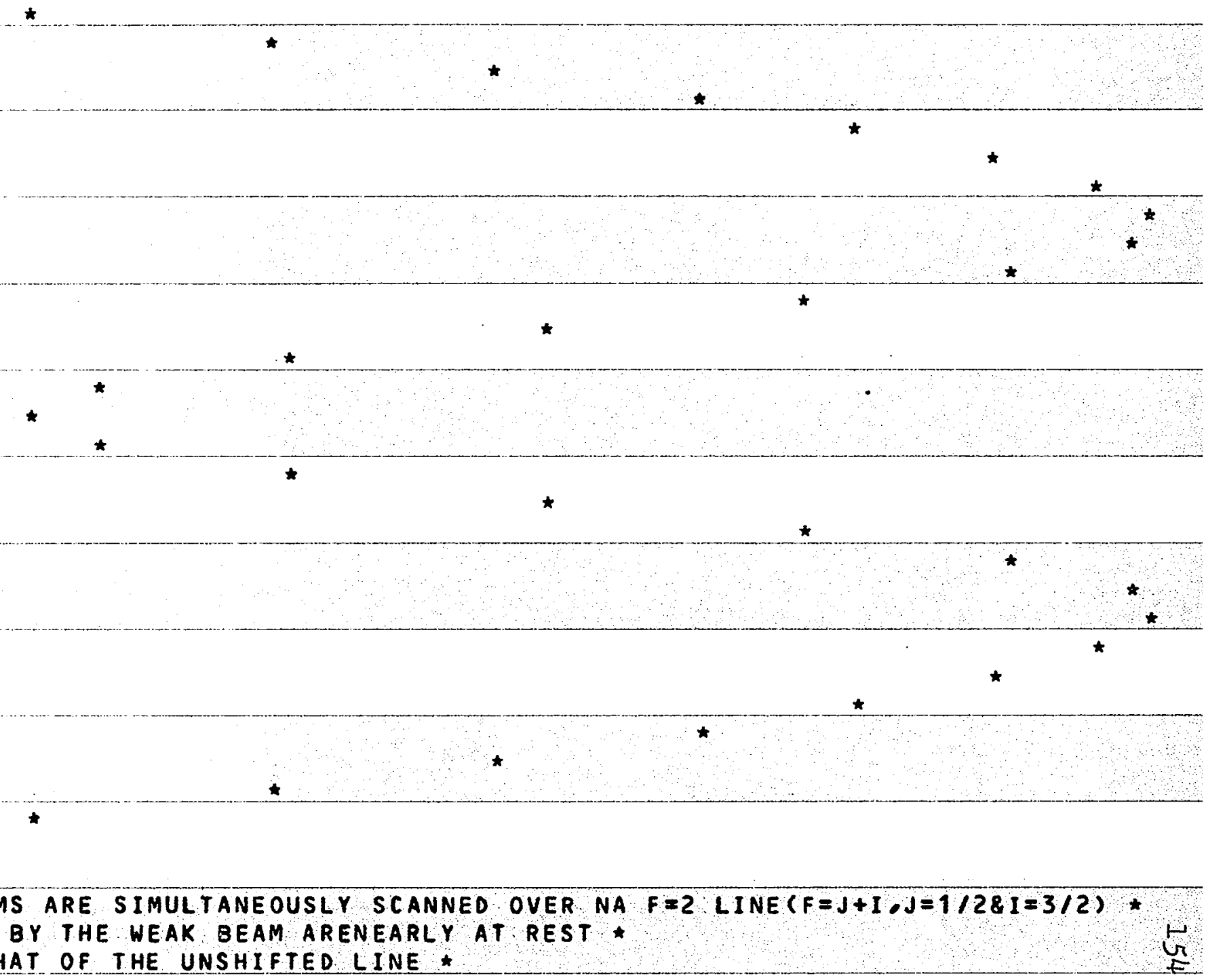


155

** * A WHITE PROBING BEAM INCIDENT ON A GLASS-NA GAS INTERFACE * * AT AN INCIDENT ANGLE OF 50.0 DEGREE

POLARIZATION IS PERPENDICULAR

* EXPERIMENTAL CONDITIONS >*

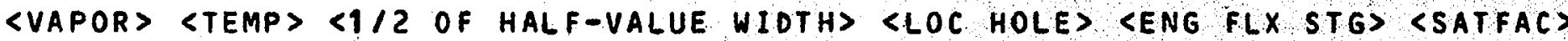
(MMHG) (KELVIN) SHWI> SHWNI> $(+10 M H Z) \quad(* 10 M H Z)$ (WATT/CM*
0.067
619.
$6.0 \quad 4.3$
0.
$0.381 E-02 \quad 0.60$

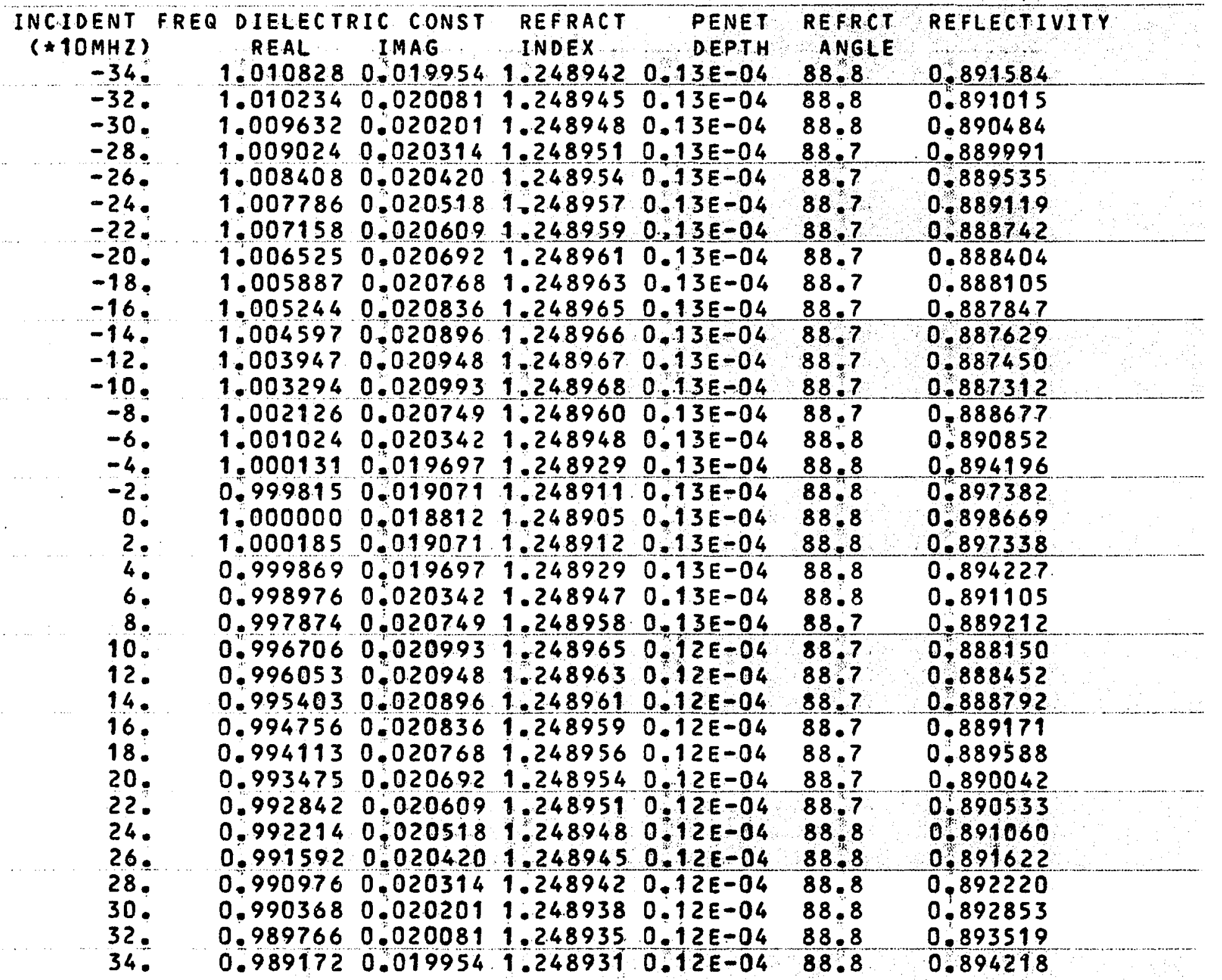


<FREQS

(*1OMHZ)

-34. 0.019954 I

$-32.0 .0200811$

$-30.0 .020201 .1$

$-28.0 .020314 I$

$-26 . \quad 0.0204201$

-24. $0.020518 \mathrm{I}$

$-22 . \quad 0.020609$ I

$-20 . \quad 0.0206921$

-18.0 .020768 I

-16.0 .020836 I

$-14,0.020896$

$-12 . \quad 0.020948$ I

$-10.0 .020993$

-8. 0.0207491

-6.0 .020342 I

$-4 . \quad 0.019697$ I

-2. 0.019071

0. $0.018812 \mathrm{I}$ *

2. 0.019071 I

4. 0.0196971

6. 0.020342 I

8. 0.0207491

10. 0.020993 I

12. 0.020948 I

14. 0.020896 I

16. 0.0208361

18. 0.020768 .

20. 0.0206921

22. $\quad 0.020609$

24. 0.0205181

26. 0.0204201

28. 0.0203141

30. 0.020201

32. 0.0200811

34. $0.019954 \quad 1$
¿IMAG PART OF THE DIELECTRIC CONSTS

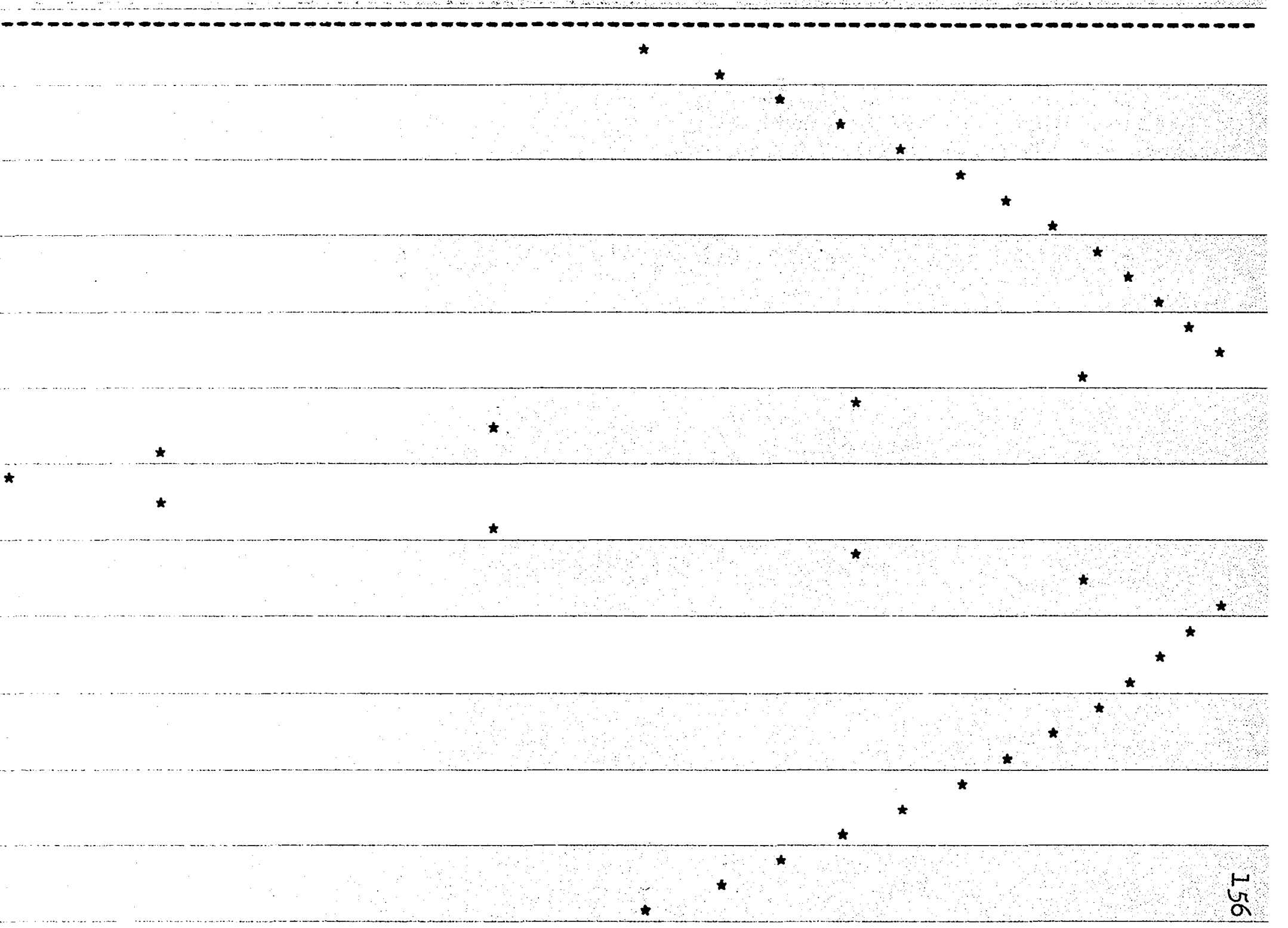

* the central freq of the strong beam is tuned to 0. *

- THEN THEASE SATURATED ATOMS ARE DETECTED BY THE WEAK WHITELIGHT RESULT IN THE PEAK AT O. * 
SFREQS

$(* 10 M H Z)$

〈REFLECTIVITYS

$-34.0 .891584 I$

$-32.0 .8910151$

$-30 . \quad 0.890484$ I

$-28,0.889991$ I

$-26.0 .8895351$

$-24.0 .8891191$

-22.0 .888742 I

$-20.0 .8884041$

$-18.0 .888105$

$-16 . \quad 0.887847 \quad$

-14.0 .887629 I

-12.0 .8874501 *

-10 . 0.887312 I*

$-8 . \quad 0.888677$ I

-6 . 0.890852 I

$-4.0 .8949961$

-2. $\quad 0.8973821$

0. 0.898669 I

2. 0.8973381

4. 0.8942271

6. 0.8911051

8. 0.889212

10. 0.888150 I

12. 0.888452 I

14. 0.8887 .92 I

16. 0.889171 I

18. $0.889588 \mathrm{I}$

20. 0.890042 I

22. $\quad 0.8905331$

24. 0.891060 I

26. 0.8916221

28. $0.892220 \quad 1$

30. 0.892853 .1

32. 0.8935191

34. 0.8942181

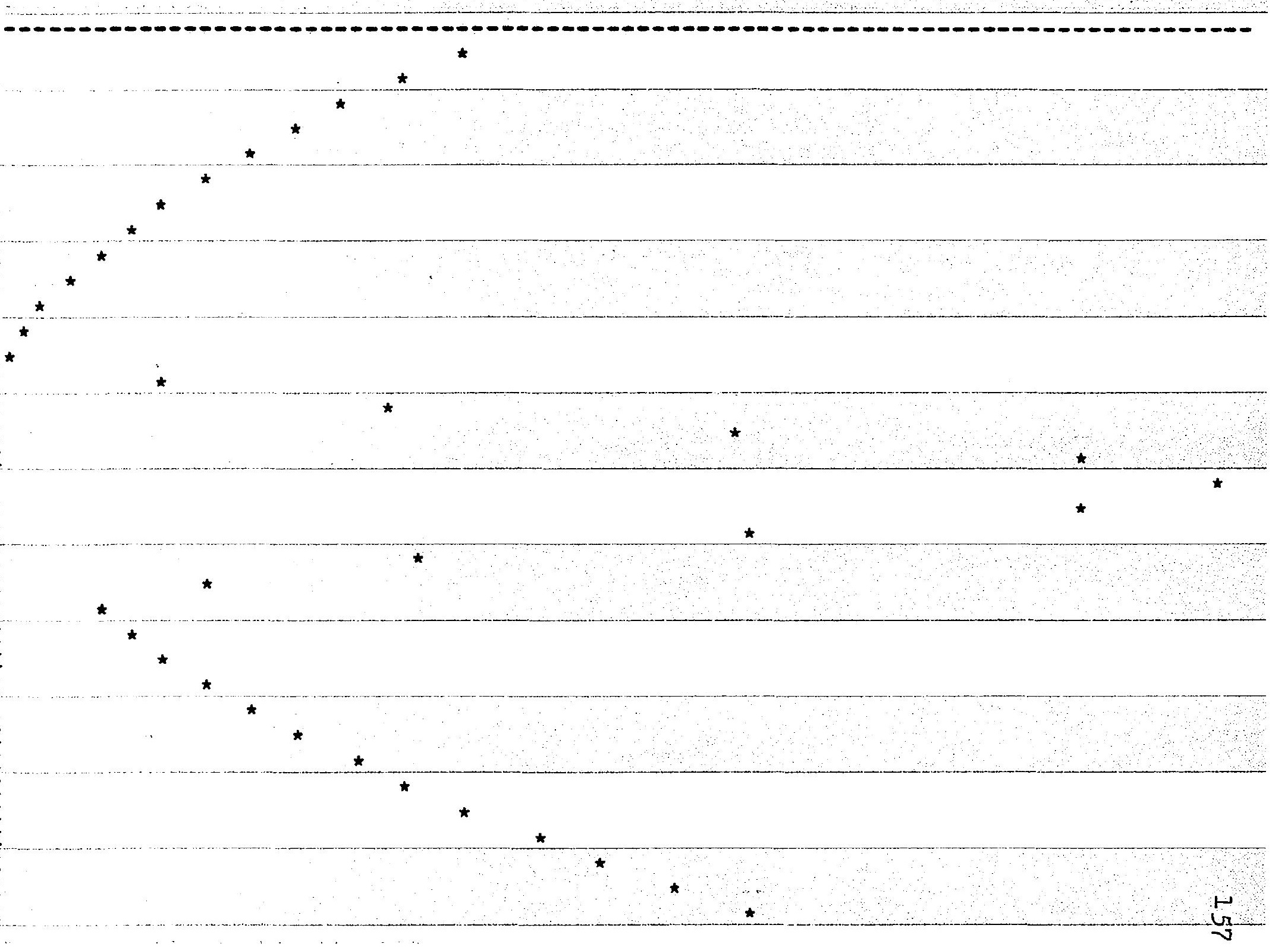

* the central freq of the strong beam is tuned to 0 . *

* THEN THEASE SATURATED ATOMS ARE DETECTED BY THE WEAK WHITE LIGHT RESULT IN THE PEAK AT O. * 


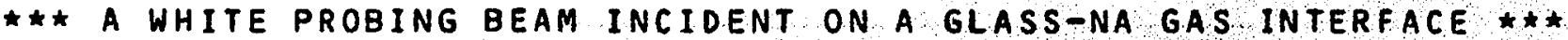
AT AN INCIDENT ANGLE OF 50.0 DEGREE

\section{POLARIZATION IS PERPENDICULAR}

$\star<$ EXPERIMENTAL CONDITIONS $>*$

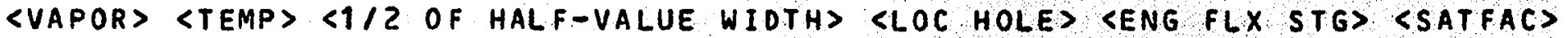
(MMHG) (KELVIN) $\angle H W I><H W N I>\quad(\star 10 M H Z)$ $(\star 10 M H Z)$ (HATT $/ C M * \star 2)$
0.067
619.
$6.0 \quad 4.3$
$-8.0$
$0.381 E-02$
0.60

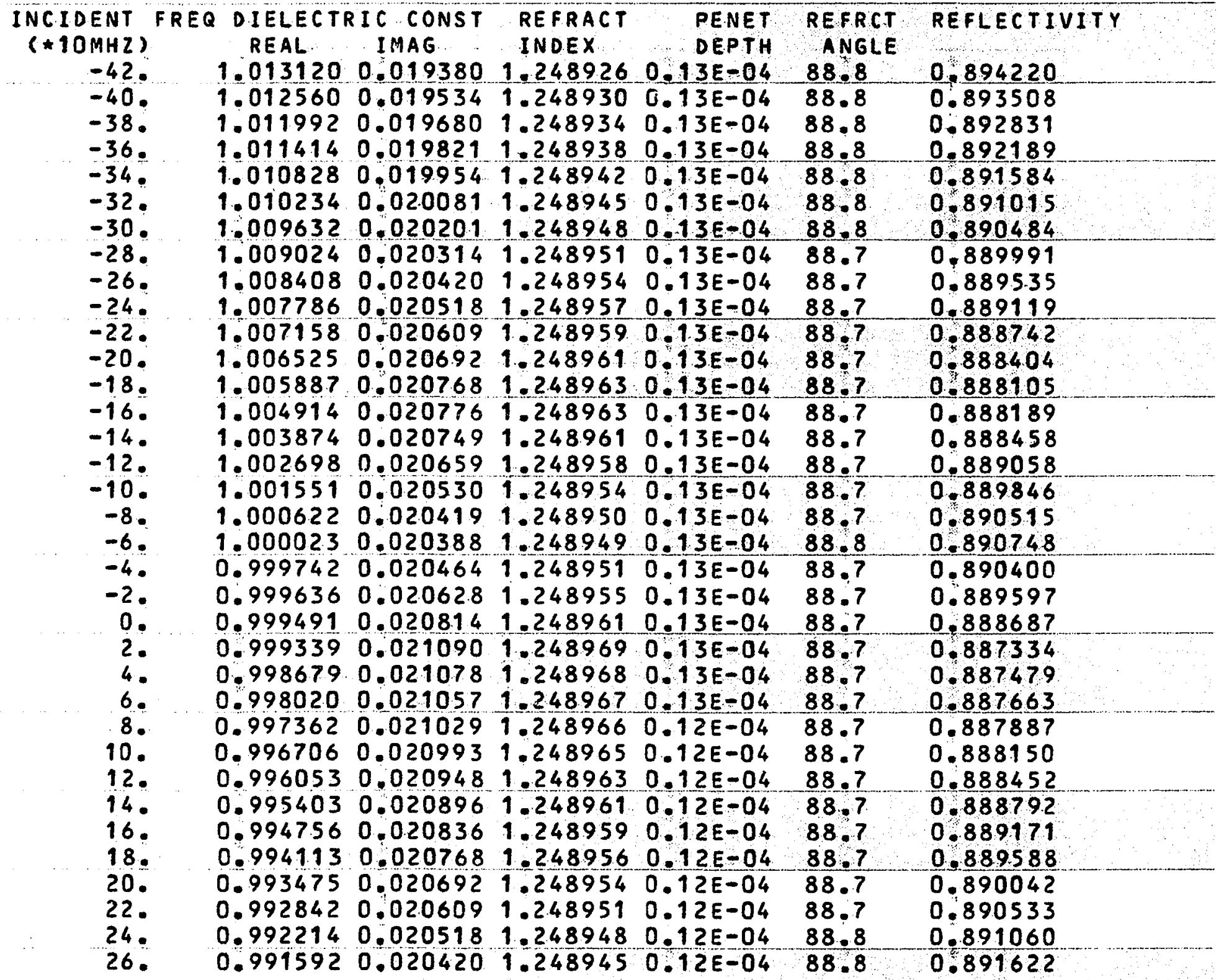




\section{$\langle F R E Q\rangle$}

¿IMAG PART OF THE DIELECTRIC CONSTS

( $(1 O M H Z)$

$-42.0 .019380 \mathrm{I}$ *

$-40 . \quad 0.019534 \mathrm{I}$

-38.0 .019680 I

-36 . 0.019821 I

$-34.0 .0199541$

$-32.0 .0200811$

$-30.0 .0202011$

$-28 . \quad 0.0203141$

$-26.0 .0204201$

$-24.0,0205181$

$-22 . \quad 0.0206091$

$-20.0 .0206921$

$-18.0 .0207681$

-16. 0.020776 I

-14.0 .020749 I

-12. 0.020659 I

$-10 . \quad 0.020530 \mathrm{I}$

-8. 0.020419 I

$-6.0 .020388$

-4. $\quad 0.020464$ I

-2. $\quad 0.0206281$

0.0 .0208141

2. $\quad 0.021090$ I

4. 0.021078 I

6. 0.021057 I

8. 0.0210291

10. 0.020993

12. 0.020948 I

14. 0.020896 I

16. 0.020836 I

18. 0.020768

20. 0.020692 I

22. 0.0206091

24. 0.020518 I

26. 0.0204201

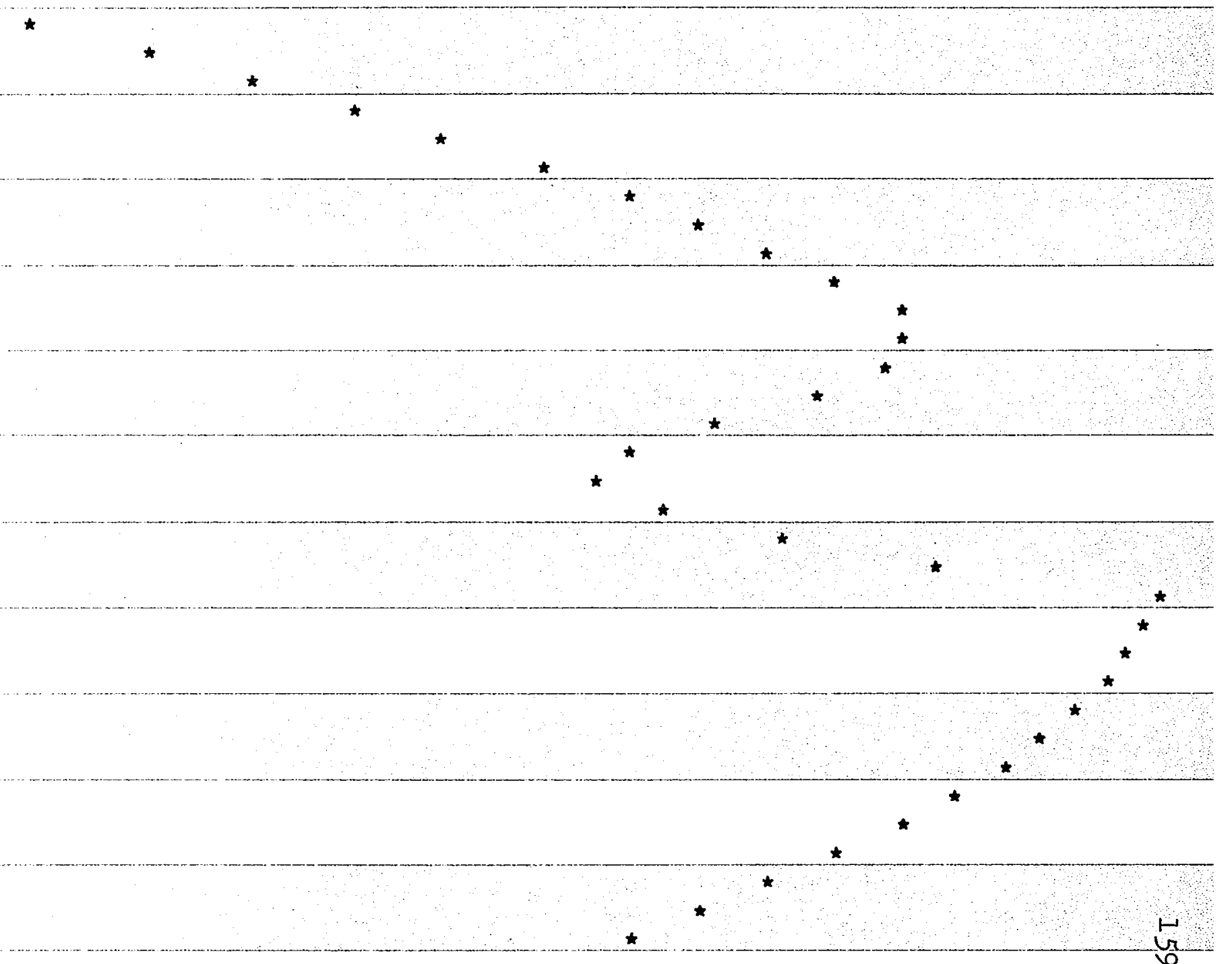

* the central freq of the strong beam is tuned to -8.0 *

* THEN THEASE SATURATED ATOMS ARE DETECTED BY THE WEAK WHITE LIGHT RESULT IN THE PEAK AT -8.0* 
- $\quad-42 . \quad 0.8942201$ $-40 . \quad 0.893508$ I $-38 . \quad 0.892831$ I $-36.0 .8921891$ -34.0 .891584 I $-32.0 .8910151$ -30.0 .890484 I $-28 . \quad 0.889991$ I $-26.0 .8895351$ $-24 . \quad 0.8891191$ $-22 . \quad 0.8887421$ $-20 . \quad 0.888404 \mathrm{I}$ -18. $0.888105 \mathrm{I}$ $-16 . \quad 0.8881891$ $-14.0 .888458 \mathrm{I}$ $-12 . \quad 0.8890581$ $-10 . \quad 0.889846$

-8.0 .890515 I

$-6.0 .890748 I$

$-4 . \quad 0.8904001$

$-2, \quad 0.889597$ I

0. 0.888687

2. $\quad 0.887334$ I

4. 0.8874791

6. 0.887663 I

8. 0.887887 . I

10. 0.888150

12. 0.888452 I

14. $\quad 0.888792$ I

16. 0.889171 . I

18. 0.8895881

20. 0.890042 I

22. 0.8905331

2.4. 0.8910601

26. 0.8916221

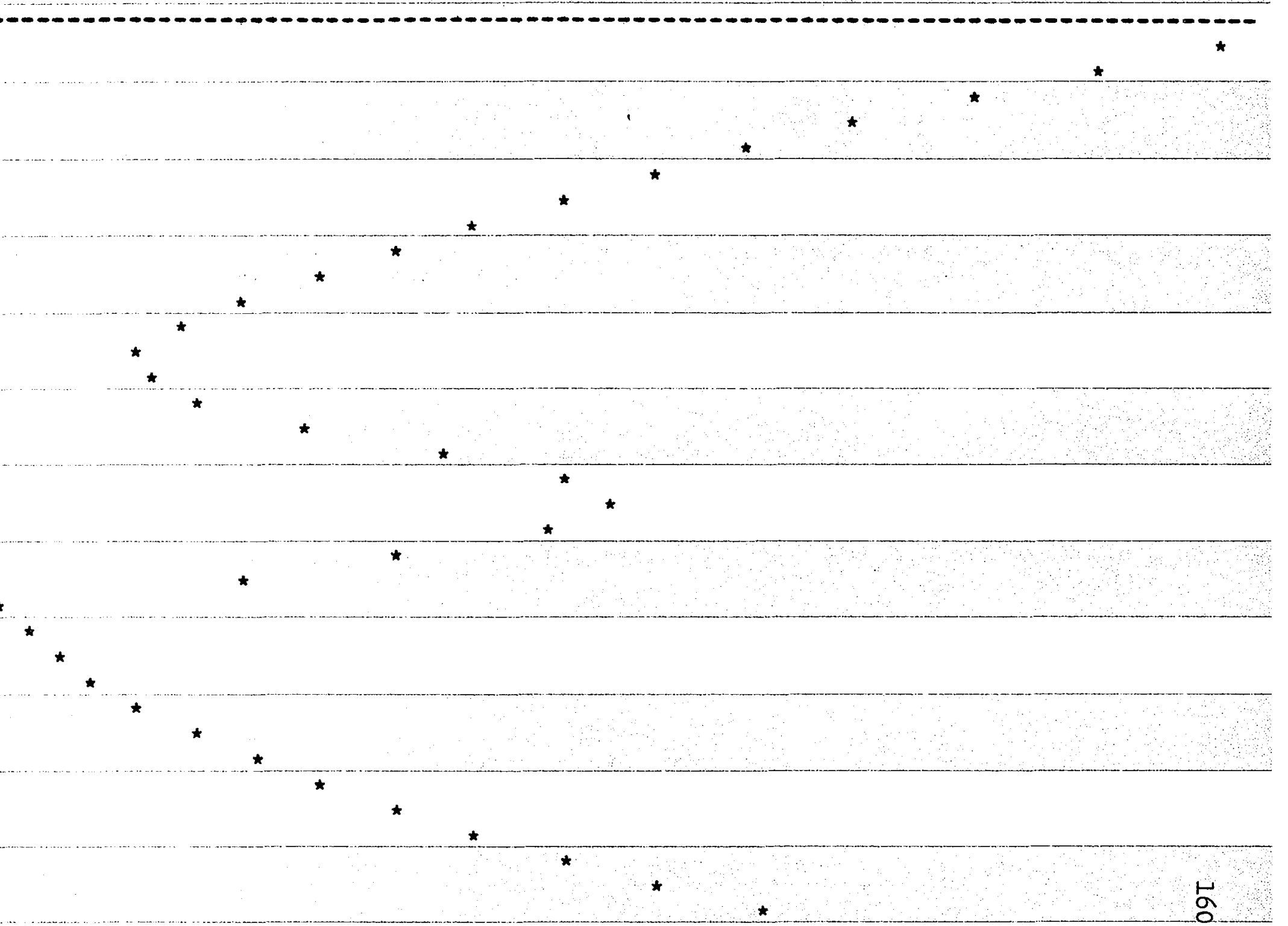

* the central freq of the strong beam is tuned to -8.0 *

* THEN THEASE SATURATED ATOMS ARE DETECTED BY THE WEAK WHITE LIGHT RESULT IN THE PEAK AT -8.O* 


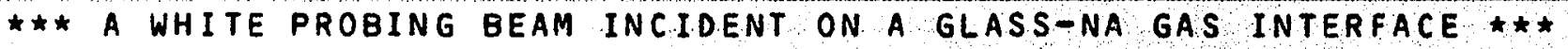

AT AN INCIDENT ANGLE OF 50.0 DEGREE

\section{POLARIZATION IS PERPENDICULAR}

$\star<$ EXPERIMENTAL CONDITIONS >

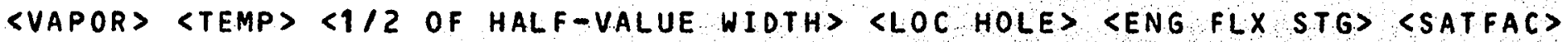
(MMHG) (KELVIN) $\quad$ SWI $\quad$ SHWNI $\quad(\star 1 O M H Z)$ $( \pm 1 O M H Z)$ (WATT $/ C M * \star 2)$
0.067
619.
6.04 .3
16.0
$0.381 E-02$
0.60

\begin{tabular}{|c|c|c|c|c|c|c|c|}
\hline $\begin{array}{r}\text { INCIDENT } \\
(\star 10 M H Z) \\
-18 \\
-16 \\
-14 \\
-12 \\
-10 \\
-8 \\
-6 \\
-4 \\
-2 \\
0 \\
2 \\
4 \\
6 \\
8 \\
10 \\
12 \\
14 \\
16 \\
18 \\
20 \\
22 \\
24 \\
26 \\
28 \\
30 \\
32 \\
34 \\
36 \\
38 \\
40 \\
42 \\
44 \\
46 \\
48 \\
50\end{array}$ & $\begin{array}{l}\text { FREQ } \\
\\
\\
0 \\
0\end{array}$ & $\begin{array}{l}\text { DIELECT } \\
\text { REAL } \\
1.005887 \\
1.005244 \\
1.004597 \\
1.003947 \\
1.003294 \\
1.002638 \\
1.001980 \\
1.001321 \\
1.000661 \\
1.000000 \\
0.999339 \\
0.998679 \\
0.998020 \\
0.997689 \\
0.997338 \\
0.997021 \\
0.996609 \\
0.996006 \\
0.995200 \\
0.994259 \\
0.993300 \\
0.992426 \\
0.991592 \\
0.990976 \\
0.990368 \\
0.989766 \\
0.989172 \\
0.988586 \\
0.988008 \\
0.987440 \\
0.986880 \\
0.986330 \\
0.985790 \\
0.985259 \\
0.984740\end{array}$ & $\begin{array}{l}\text { RIC CONST } \\
\text { IMAG } \\
0.020768 \\
0.020836 \\
0.020896 \\
0.020948 \\
0.020993 \\
0.021029 \\
0.021057 \\
0.021078 \\
0.021090 \\
0.021094 \\
0.021090 \\
0.021078 \\
0.021057 \\
0.020969 \\
0.020883 \\
0.020787 \\
0.020703 \\
0.020647 \\
0.020615 \\
0.020592 \\
0.020555 \\
0.020495 \\
0.020420 \\
0.020314 \\
0.020201 \\
0.020081 \\
0.019954 \\
0.019821 \\
0.019680 \\
0.019534 \\
0.019380 \\
0.019221 \\
0.019056 \\
0.018885 \\
0.018708\end{array}$ & $\begin{array}{l}\text { REFRACT } \\
\text { INDEX } \\
1.248963 \\
1.248965 \\
1.248966 \\
1.248967 \\
1.248968 \\
1.248969 \\
1.248969 \\
1.248970 \\
1.248970 \\
1.248969 \\
1.248969 \\
1.248968 \\
1.248967 \\
1.248964 \\
1.248962 \\
1.248959 \\
1.248956 \\
1.248954 \\
1.248953 \\
1.248951 \\
1.248950 \\
1.248948 \\
1.248945 \\
1.248942 \\
1.248938 \\
1.248935 \\
1.248931 \\
1.248927 \\
1.248923 \\
1.248918 \\
1.248914 \\
1.248909 \\
1.248905 \\
1.248900 \\
1.248895\end{array}$ & $\begin{array}{r}\text { PENET } \\
\text { DEPJH } \\
0.13 E-04 \\
0.13 E-04 \\
0.13 E-04 \\
0.13 E-04 \\
0.13 E-04 \\
0.13 E-04 \\
0.13 E-04 \\
0.13 E-04 \\
0.13 E-04 \\
0.13 E-04 \\
0.13 E-04 \\
0.13 E-04 \\
0.13 E-04 \\
0.13 E-04 \\
0.12 E-04 \\
0.12 E-04 \\
0.12 E-04 \\
0.12 E-04 \\
0.12 E-04 \\
0.12 E-04 \\
0.12 E-04 \\
0.12 E-04 \\
0.12 E-04 \\
0.12 E-04 \\
0.12 E-04 \\
0.12 E-04 \\
0.12 E-04 \\
0.12 E-04 \\
0.12 E-04 \\
0.12 E-04 \\
0.12 E-04 \\
0.12 E-04 \\
0.12 E-04 \\
0.12 E-04 \\
0.12 E-04\end{array}$ & $\begin{array}{l}\text { REFRCT } \\
\text { ANGLE } \\
88.7 \\
88.7 \\
88.7 \\
88.7 \\
88.7 \\
88.7 \\
88.7 \\
88.7 \\
88.7 \\
88.7 \\
88.7 \\
88.7 \\
88.7 \\
88.7 \\
88.7 \\
88.7 \\
88.7 \\
88.7 \\
88.7 \\
88.7 \\
88.7 \\
88.8 \\
88.8 \\
88.8 \\
88.8 \\
88.8 \\
88.8 \\
88.8 \\
88.8 \\
88.8 \\
88.8 \\
88.8 \\
88.8 \\
88.9 \\
88.9 \\
8 \\
8 \\
8\end{array}$ & $\begin{array}{l}\text { REFLECTIVITY } \\
0.888105 \\
0.887847 \\
0.8876 .29 \\
0.887450 \\
0.887312 \\
0.887215 \\
0.887158 \\
0.887141 \\
0.887165 \\
0.887230 \\
0.887334 \\
0.887479 \\
0.887663 \\
0.888143 \\
0.888615 \\
0.889131 \\
0.889598 \\
0.889954 \\
0.890210 \\
0.890445 \\
0.890744 \\
0.891148 \\
0.891622 \\
0.892220 \\
0.892853 \\
0.893519 \\
0.894218 \\
0.89 .4950 \\
0.895714 \\
0.896508 \\
0.897333 \\
0.898187 \\
0.899069 \\
0.899979 \\
0.900916 \\
0.890\end{array}$ \\
\hline
\end{tabular}




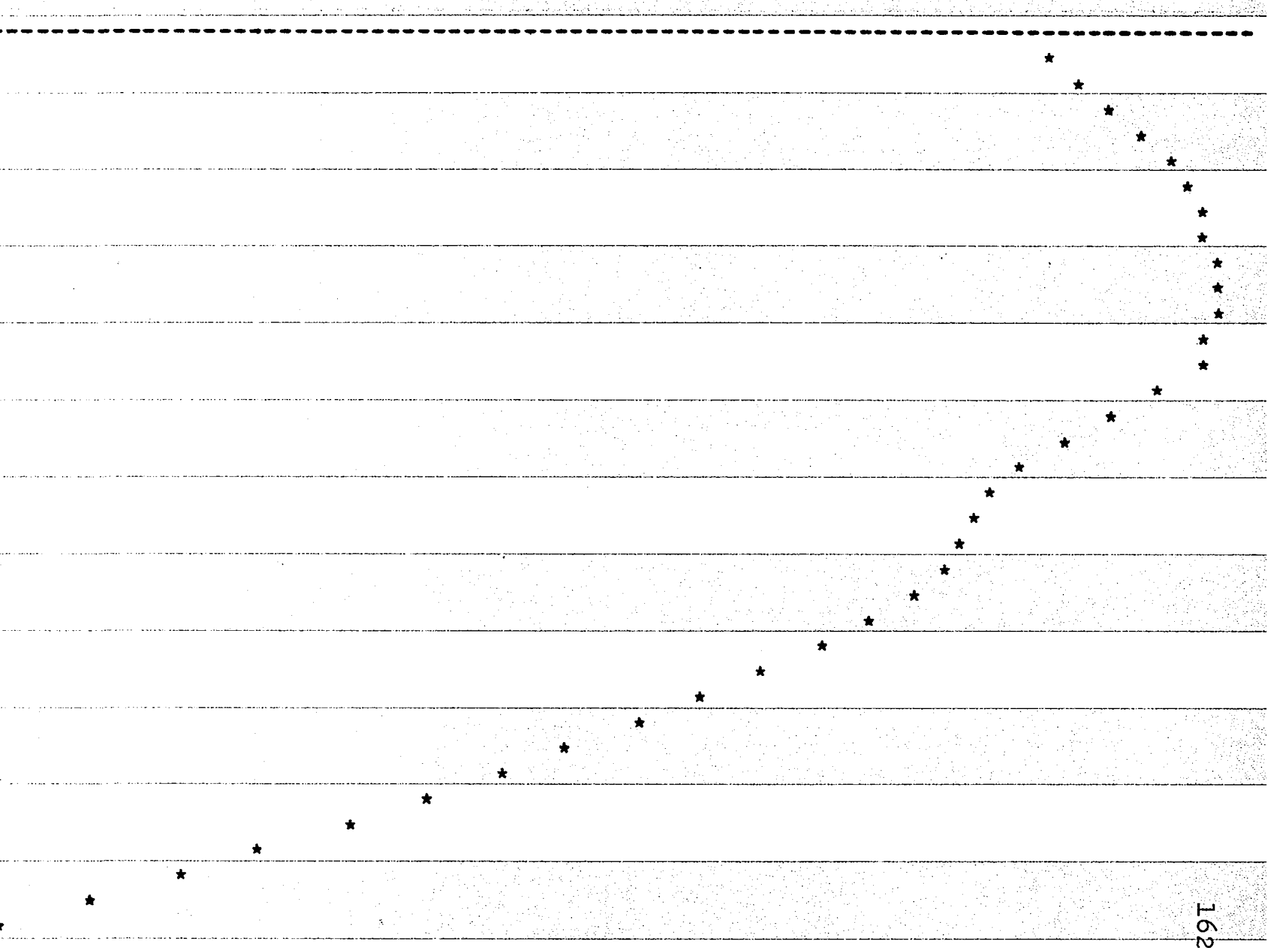

* the central freq of the strong beam is tUNEd to 16.0 *

* THEN THEASE SATURATED ATOMS ARE DETECTED BY THE WEAK WHITE LIGHT RESULT IN THE PEAK AT 16.0 * 
(*1OMHZ)

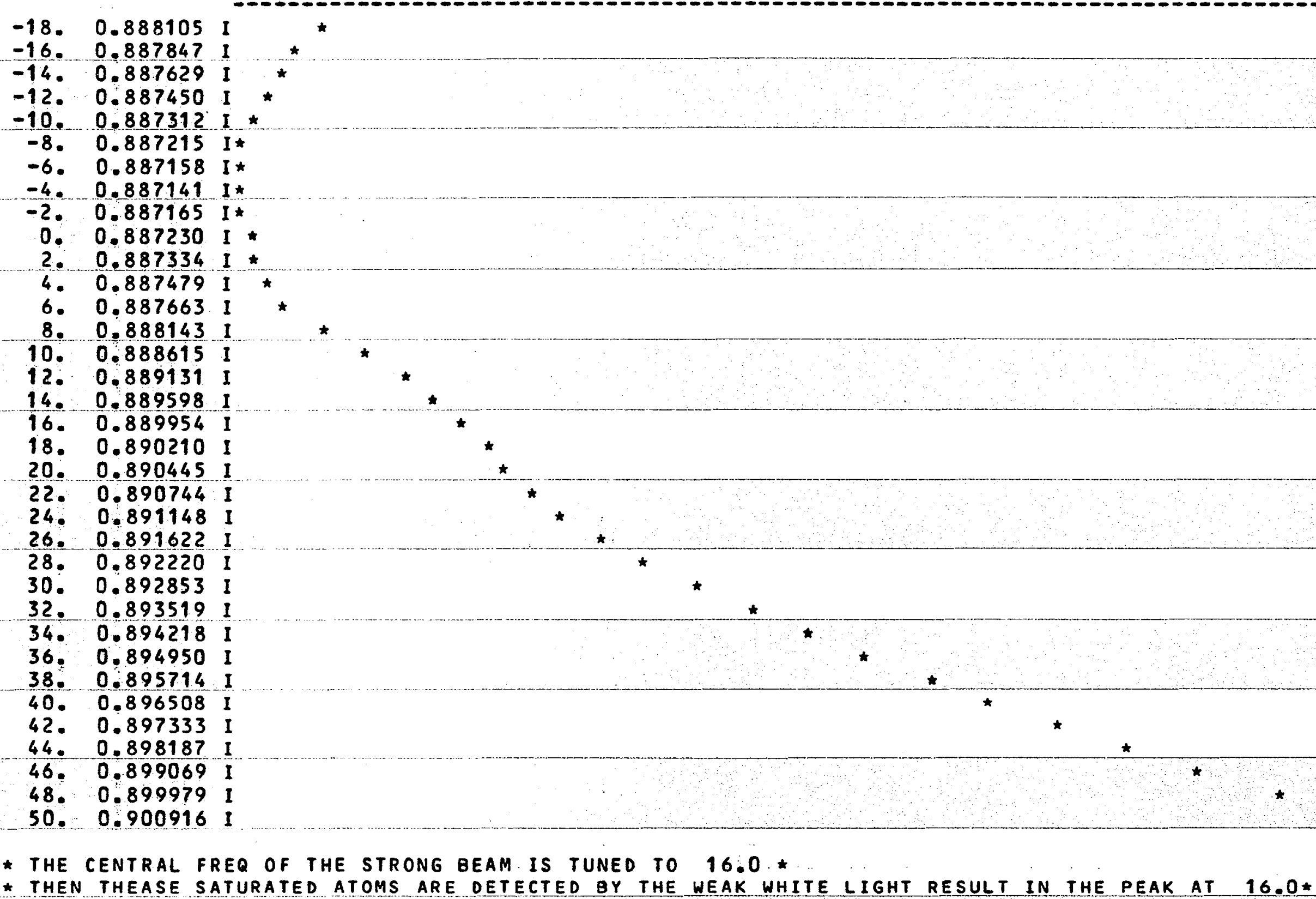

* THEN THEASE SATURATED ATOMS ARE DETECTED BY THE WEAK WHITE LIGHT RESULT IN THE PEAK AT 16.0* 


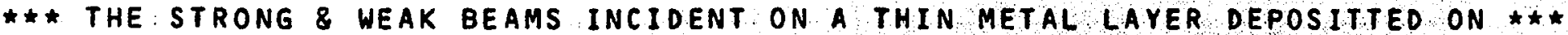
A GLASS-NA GAS INTERFACE AT AN INCIDENT ANGLE OF 50.0 OEGREE

\section{POLARIZATION IS PERPENDICULAR}

* VAN DER WAAL 'S POTENTIAL ENERGY HAS THE FORM OF THE INVERSE * * $3 R D$ POWER OF THE NORMAL DISTANCE FROM THE SURFACE WITH A CONSTANT 4.0 IN UNIT OF E-12*ERG PER A* 3

*< EXPERIMENTAL CONDITIONS >*

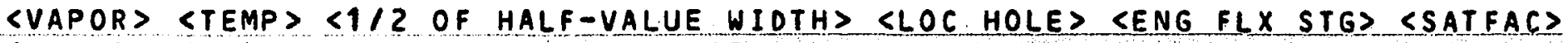
(MMHG) (KELVIN) SHWI> SHWNIS (
0.067
619.
$6.0 \quad 4.3$
$-40.0$
$0.381 E-02$
0.60

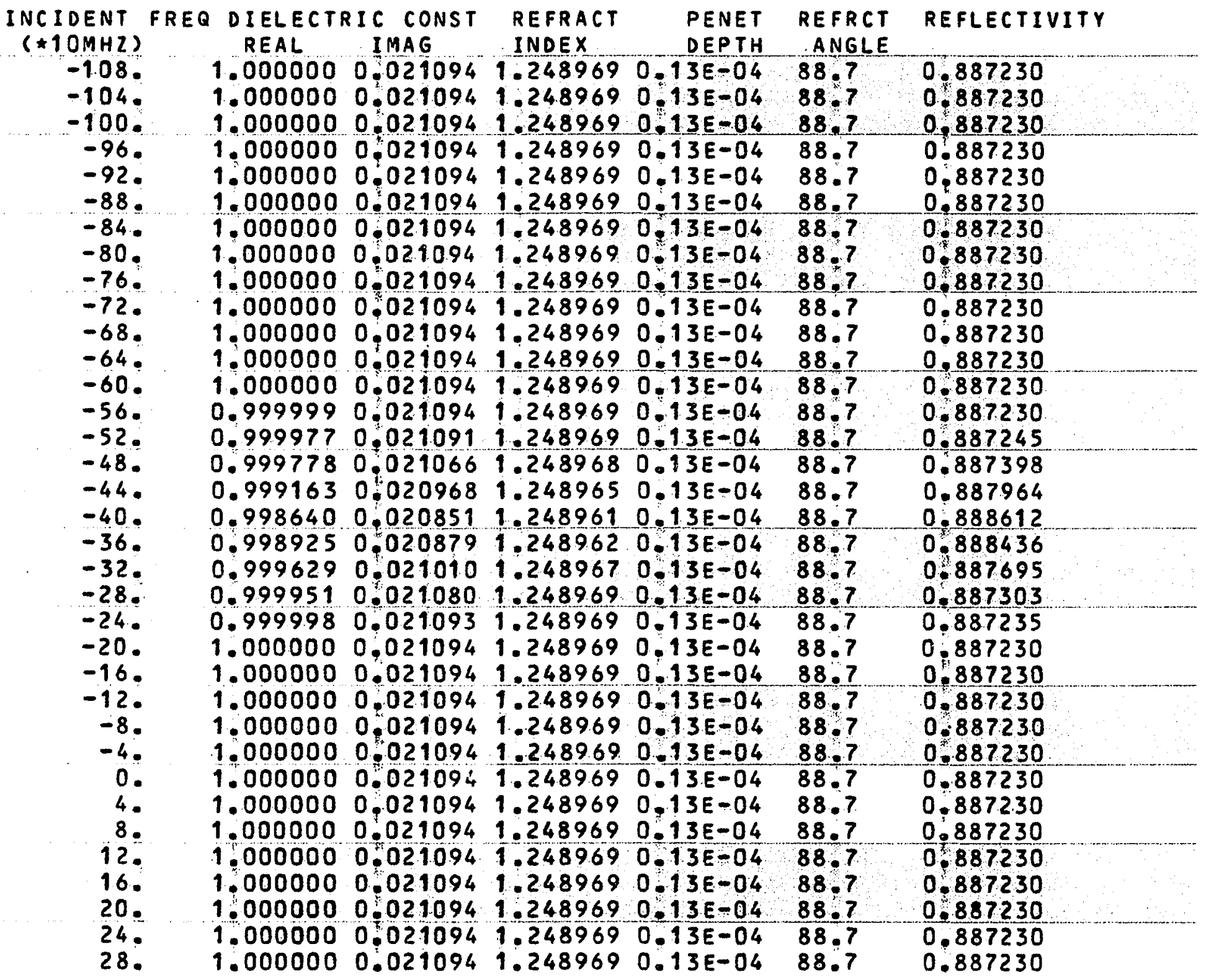


( $\star 1 O M H Z)$

- -108. 0.887230 I*

$-104,0.887230$ I*

-100.0 .887230 I*

-96. $0.887230 I$ I*

-92 . 0.887230 I*

-88. $0.887230 I$ I*

-84 . $0.887230 I$ I*

-80 . 0.8872301 I*

-76. 0.887230 I*

$-72.0 .887230 \mathrm{I}$ *

-68 . 0.887230 I*

-64 . 0.887230 I*

-60 . 0.887230 I*

-56 . 0.887230 I*

-52. $0.887245 I$ *

$-48 . \quad 0.887398$ I

-44 . 0.887964 I

-40.0 .888612 I

$-36.0 .888436$

-32 . $\quad 0.887695$ I

-28.0 .887303 I

-24. $0.887235 I$ *

-20.0 .887 .230 I*

-16. 0.887230 I*

-12. $0.8872301 *$

-8. 0.887230 I*

-4 . 0.887 .230 I*

0. 0.887230 I*

4. 0.887230 I*

8. 0.8872301 I*

12. $0.887 .230 \mathrm{I*}$

16. $0.887230 \mathrm{I*}$

20. $0.887 .230 I *$

24. $0.8872301 *$

28. $0.8872301 *$

THE CENTRAL. FREQ OF THE STRONG BEAM IS TUNED TO -40.0 *

THEN THEASE SATURATED ATOMS UNDER THE WALL INTERACTIONS ARE DETECTED BY WHITE LIGHT * 


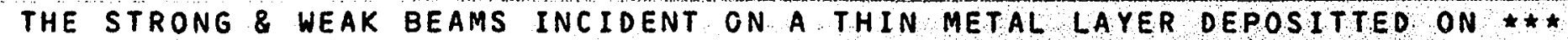
A GLASS-NA GAS INTERFACE AT AN INCIDENT ANGLE OF 50.0 DEGREE

\section{POLARIZATION IS PERPENDICULAR}

* VAN DER WAAL'S POTENTIAL ENERGY HAS THE FORM OF THE INVERSE * * 4 TH POWER OF THE NORMAL DISTANCE FROM THE SURFACE

WITH A CONSTANT $20: 0$ IN UNIT OF E-12 $\#$ ERG PER A* $* 4$

$$
\text { * }<\text { EXPERIMENTAL CONDITIONS }>*
$$

SVAPOR> <TEMP > $\angle 1 / 2$ OF HALF-VALUE WIDTH> SMIN REFL OCCURED AT FREQ (MMHG) (KELVIN) $\angle H W I><H W N 1\rangle(\star 10 M H Z)$ (*E12HZ)

$0.067 \quad 619.6 .0 \quad 4.3 \quad 62.82 \quad-726$

LINE SHAPE OF THE HOLE ISN'T EXAMINED

\begin{tabular}{|c|c|c|c|c|c|c|c|}
\hline $\begin{array}{c}\text { INCIDENT } \\
(\star E 12 H Z) \\
-73 \\
-69 \\
-65 \\
-61 \\
-57 \\
-53 \\
-49 \\
-45 \\
-41 \\
-37 \\
-33 \\
-29 \\
-25 \\
-21 \\
-17 \\
-13 \\
-9 \\
-5\end{array}$ & 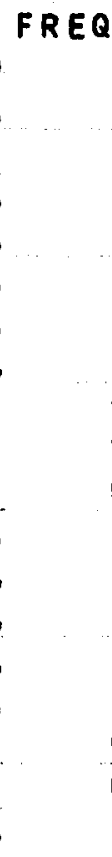 & $\begin{array}{l}\text { DIELECTR } \\
\text { REAL } \\
0.999296 \\
0.999317 \\
0.999338 \\
0.999358 \\
0.999377 \\
0.999396 \\
0.999415 \\
0.999432 \\
0.999450 \\
0.999466 \\
0.999483 \\
0.999498 \\
0.999514 \\
0.999528 \\
0.999543 \\
0.999557 \\
0.999570 \\
0.999583\end{array}$ & $\begin{array}{l}\text { RIC CONST } \\
\text { IMAG } \\
0.005512 \\
0.005345 \\
0.005182 \\
0.005025 \\
0.004872 \\
0.004724 \\
0.004581 \\
0.004442 \\
0.004307 \\
0.004176 \\
0.004049 \\
0.003926 \\
0.003807 \\
0.003691 \\
0.003579 \\
0.003470 \\
0.003365 \\
0.003262\end{array}$ & $\begin{array}{l}\text { REFRACT } \\
\text { INDEX } \\
1.248673 \\
1.248672 \\
1.248671 \\
1.248670 \\
1.248669 \\
1.248668 \\
1.248667 \\
1.248666 \\
1.248665 \\
1.248664 \\
1.248663 \\
1.248663 \\
1.248662 \\
1.248661 \\
1.248661 \\
1.248660 \\
1.248660 \\
1.248659\end{array}$ & $\begin{array}{l}\text { PENET } \\
\text { DEPTH } \\
0.13 E-04 \\
0.13 E-04 \\
0.13 E-04 \\
0.13 E-04 \\
0.13 E-04 \\
0.13 E-04 \\
0.13 E-04 \\
0.13 E-04 \\
0.13 E-04 \\
0.13 E-04 \\
0.13 E-04 \\
0.13 E-04 \\
0.13 E-04 \\
0.13 E-04 \\
0.13 E-04 \\
0.13 E-04 \\
0.13 E-04 \\
0.13 E-04\end{array}$ & $\begin{array}{l}\text { REFRCT } \\
\text { ANGLE } \\
89.7 \\
89.7 \\
89.7 \\
89.7 \\
89.7 \\
89.7 \\
89.7 \\
89.7 \\
89.7 \\
89.7 \\
89.8 \\
89.8 \\
89.8 \\
89.8 \\
89.8 \\
89.8 \\
89.8 \\
89.8\end{array}$ & $\begin{array}{l}\text { REFLECTIVITY } \\
0.968991 \\
0.9 .69916 \\
0.970812 \\
0.971684 \\
0.972530 \\
0.973351 \\
0.974147 \\
0.974922 \\
0.975670 \\
0.976399 \\
0.977106 \\
0.977792 \\
0.978458 \\
0.979105 \\
0.979732 \\
0.980340 \\
0.980931 \\
0.981504\end{array}$ \\
\hline
\end{tabular}


〈FREQS

$(* E+13 H Z)$

〈REFLECTIVITYS

$-72.0 .968991 .1 *$

$-68.0 .969916 I$

$-64 . \quad 0.970812$ I

$-60.0 .971684 I$

$-56 . \quad 0.972530$

-52.0 .973351 I

-48.0 .974147 I

$-44.0 .9749221$

$-40 . \quad 0.975670$ I

$-36.0 .97639 .91$

$-32 . \quad 0.977106$

$-28 . \quad 0.977792$ I

-24.0 .978458 I

$-20 . \quad 0.9791051$

$-16.0 .979732$

$-12.0 .980340 \mathrm{I}$

-8. 0.980931

-4.0 .981504 I

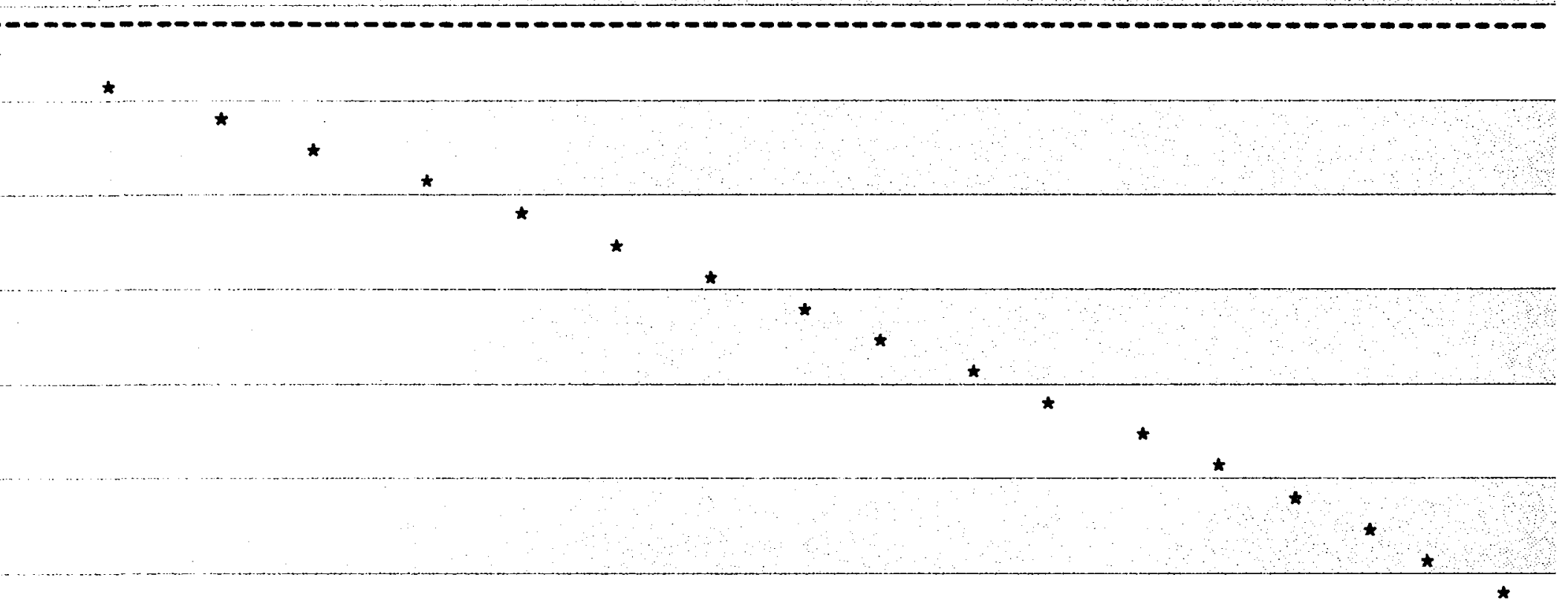

* THE NARROW STRONG \& WEAK BEAMS ARE SIMULTANEOUSLY SCANNED OVER NA F=2 LINE(F=J+I,J=1/2\&I=3/2) *

* THE SATURATED aTOMS DETECTED ARE NEARLY at REST *

*THE DISTRIBUTION OF THE PEAKS (DUE TO THE HOLES) IS PLOTTED * 
168

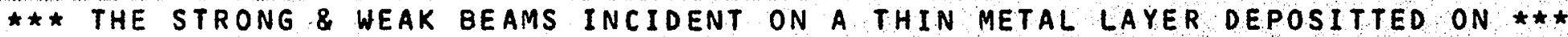
A GLASS-NA GAS INTERFACE AT AN INCIDENT ANGLE OF 50.0 DEGREE

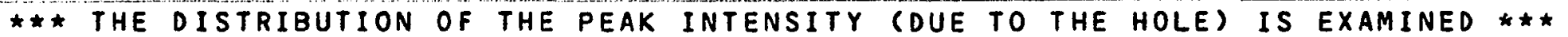

POLARIZATION IS PERPENDICULAR

* * VAN DER WAAL'S POTENTIAL ENERGY HAS THE FORM OF THE INVERSE *

3RD POWER OF THE NORMAL DISTANCE FROM THE SURFACE

WITH A CONSTANT 4.0 IN UNIT OF E-12*ERG PER $A * \star 3$

$\star<$ EXPERIMENTAL CONDITIONS $>\star$

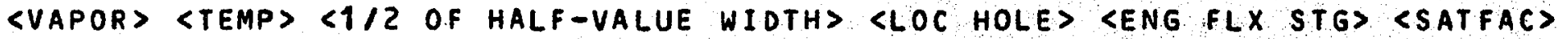
(MMHG) (KELVIN) <HWI> SHWNI> $(\star 10 M H Z) \quad(\star 10 M H Z)$ (WATT/CM $\star 2)$

$\begin{array}{llllll}0.067 & 619 . & 20.0 & 4.3 & 0.381 E-02 & 0.60\end{array}$

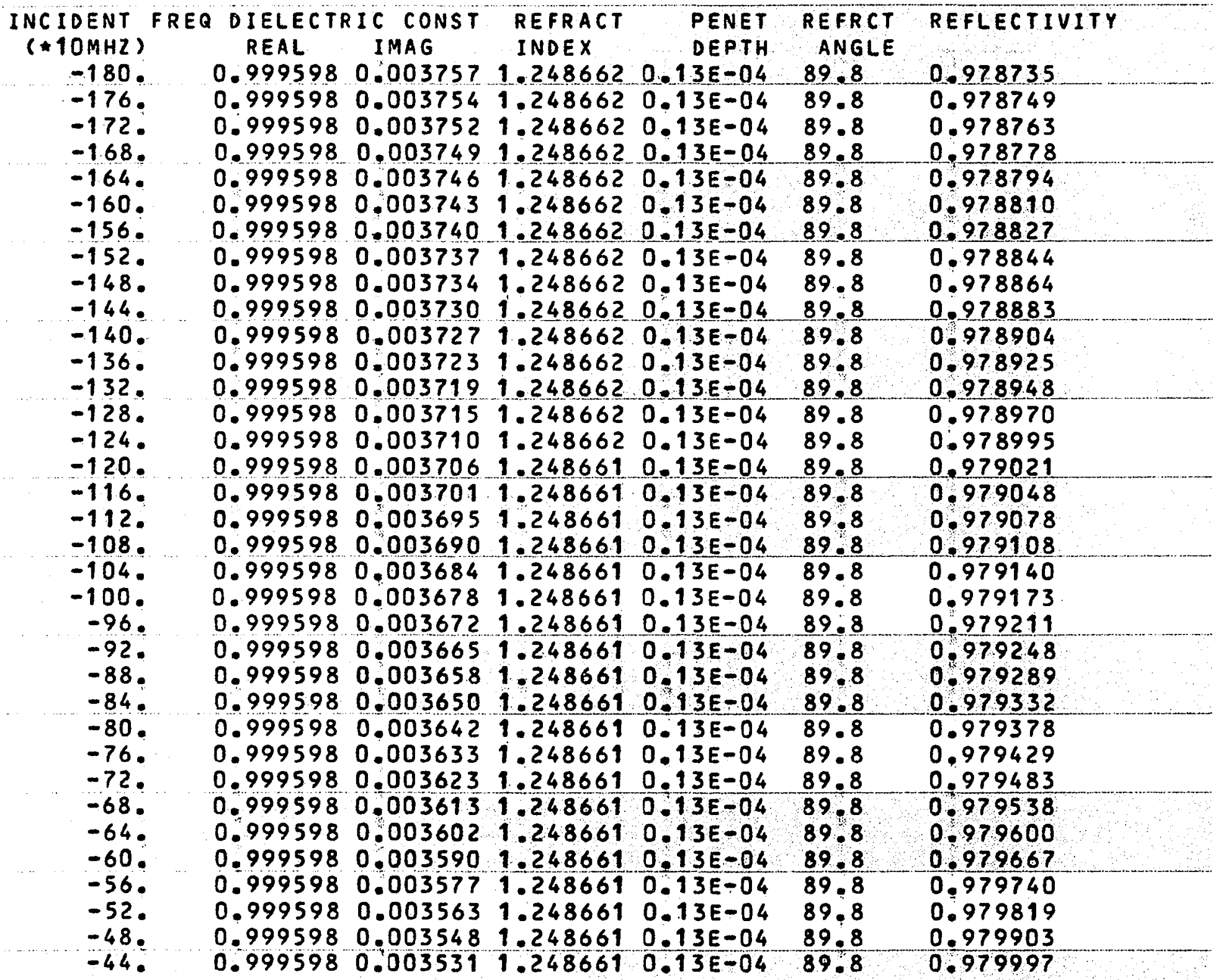




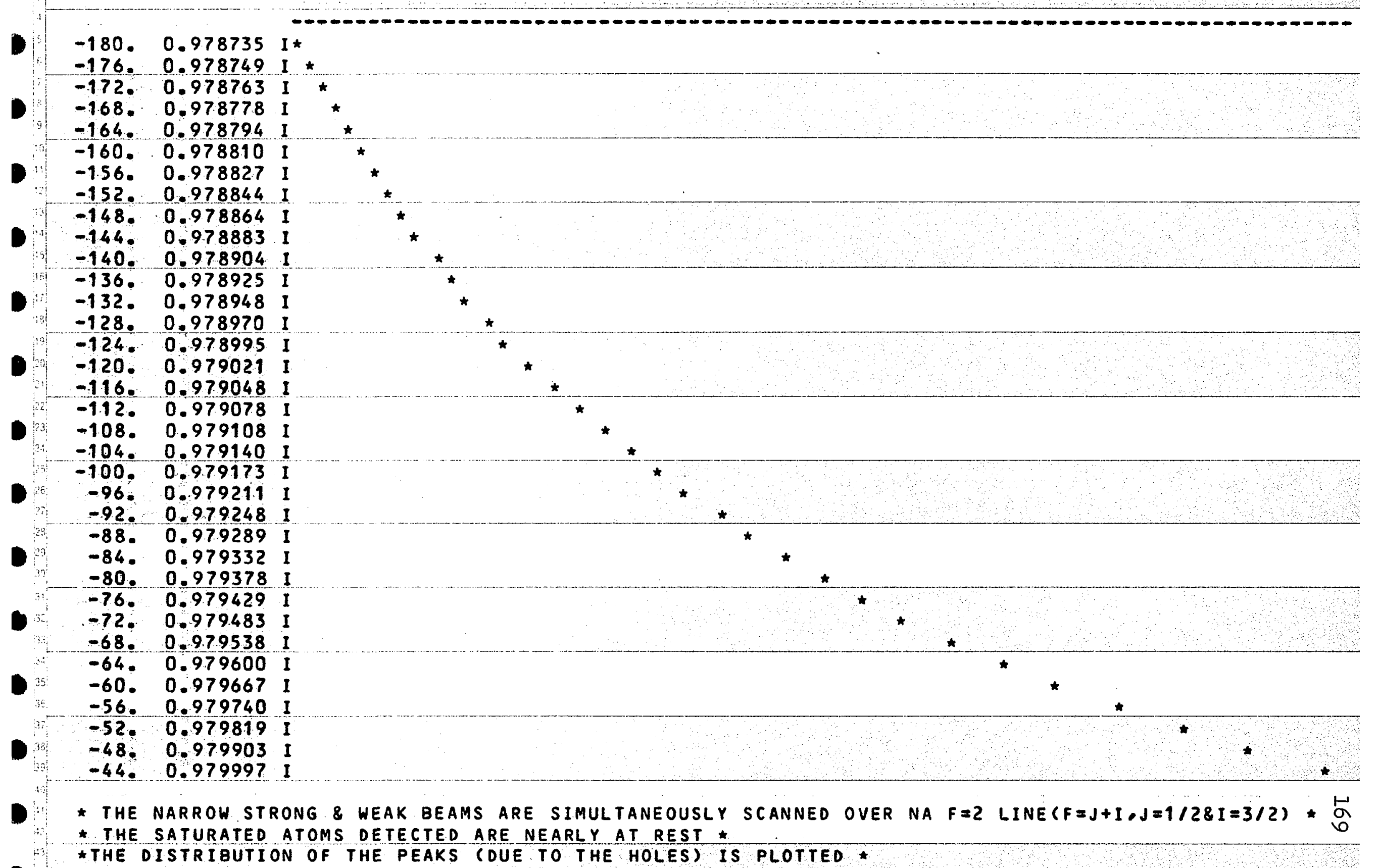


$\star \star *$ THE STRONG \& WEAK BEAMS INCIDENT ON A THIN METAL LAYER DEPOSITTED ON *** A GLASS-NA GAS INTERFACE AT AN INCIDENT ANGLE OF 50.0 DEGREE

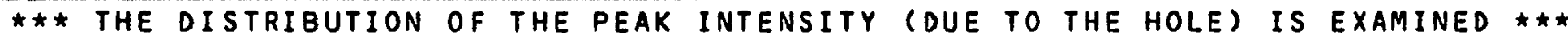

POLARIZATION IS PERPENDICULAR

* * VAN DER WAAL'S POTENTIAL ENERGY HAS THE FORM OF THE INVERSE * * 2ND POWER OF THE NORMAL DISTANCE FROM THE SURFACE WITH A CONSTANT 0.8 IN UNIT OF E-12*ERG PER $A \star 2$

* EXPERIMENTAL CONDITIONS >*

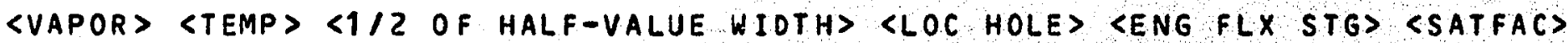
(MMHG) (KELVIN) <HWI> SHWNI $(\star 10 M H Z) \quad(\star 10 M H Z)$ $(W A T T / C M * \star 2)$

$\begin{array}{llllll}0.067 & 619 . & 20.0 & 4.3 & 0.381 E-02 & 0.60\end{array}$

\begin{tabular}{|c|c|c|c|c|c|c|c|}
\hline $\begin{array}{r}\text { INCI DENT } \\
(* 10 M H Z) \\
-180 \\
-176 \\
-172 \\
-168 \\
-164 \\
-160 \\
-156 \\
-152 \\
-148 \\
-144 \\
-140 \\
-136 \\
-132 \\
-128 \\
-124 \\
-120 \\
-116 \\
-112 \\
-108 \\
-104 \\
-100 \\
-96 \\
-92 \\
-88 \\
-84 \\
-80 \\
-76 \\
-72 \\
-68 \\
-64 \\
-60 \\
-56 \\
-52 \\
-48 \\
-44\end{array}$ & 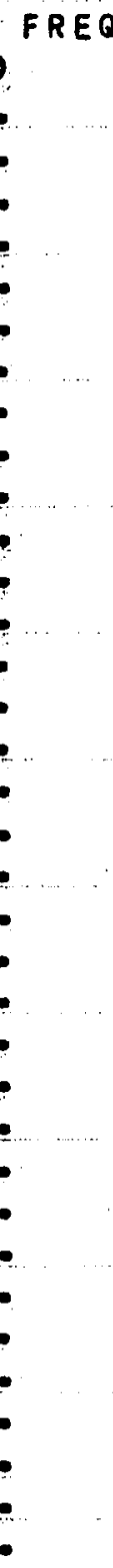 & $\begin{array}{l}\text { DIELECTF } \\
\text { REAL } \\
0.999598 \\
0.999598 \\
0.999598 \\
0.999598 \\
0.999598 \\
0.999598 \\
0.999598 \\
0.999598 \\
0.999598 \\
0.999598 \\
0.999598 \\
0.999598 \\
0.999598 \\
0.999598 \\
0.999598 \\
0.999598 \\
0.999598 \\
0.999598 \\
0.999598 \\
0.999598 \\
0.999598 \\
0.999598 \\
0.999598 \\
0.999598 \\
0.999598 \\
0.999598 \\
0.999598 \\
0.999598 \\
0.999598 \\
0.999598 \\
0.999598 \\
0.999598 \\
0.999598 \\
0.999598 \\
0.999598\end{array}$ & $\begin{array}{l}\text { RIC CONST } \\
\text { IMAG } \\
0.003801 \\
0.003800 \\
0.003798 \\
0.003796 \\
0.003794 \\
0.003792 \\
0.003790 \\
0.003788 \\
0.003785 \\
0.003783 \\
0.003780 \\
0.003778 \\
0.003775 \\
0.003772 \\
0.003769 \\
0.003766 \\
0.003762 \\
0.003758 \\
0.003754 \\
0.003750 \\
0.003746 \\
0.003741 \\
0.003736 \\
0.003731 \\
0.003725 \\
0.003719 \\
0.003712 \\
0.003705 \\
0.003697 \\
0.003689 \\
0.003680 \\
0.003670 \\
0.003659 \\
0.003647 \\
0.003634\end{array}$ & $\begin{array}{l}\text { REFRACT } \\
\text { INDEX } \\
1.248662 \\
1.248662 \\
1.248662 \\
1.248662 \\
1.248662 \\
1.248662 \\
1.248662 \\
1.248662 \\
1.248662 \\
1.248662 \\
1.248662 \\
1.248662 \\
1.248662 \\
1.248662 \\
1.248662 \\
1.248662 \\
1.248662 \\
1.248662 \\
1.248662 \\
1.248662 \\
1.248662 \\
1.248662 \\
1.248662 \\
1.248662 \\
1.248662 \\
1.248662 \\
1.248662 \\
1.248661 \\
1.248661 \\
1.248661 \\
1.248661 \\
1.248661 \\
1.248661 \\
1.248661 \\
1.248661\end{array}$ & $\begin{array}{r}\text { PENET } \\
\text { DEPTH } \\
0.13 E-04 \\
0.13 E-04 \\
0.13 E-04 \\
0.13 E-04 \\
0.13 E-04 \\
0.13 E-04 \\
0.13 E-04 \\
0.13 E-04 \\
0.13 E-04 \\
0.13 E-04 \\
0.13 E-04 \\
0.13 E-04 \\
0.13 E-04 \\
0.13 E-04 \\
0.13 E-04 \\
0.13 E-04 \\
0.13 E-04 \\
0.13 E-04 \\
0.13 E-04 \\
0.13 E-04 \\
0.13 E-04 \\
0.13 E-04 \\
0.13 E-04 \\
0.13 E-04 \\
0.13 E-04 \\
0.13 E-04 \\
0.13 E-04 \\
0.13 E-04 \\
0.13 E-04 \\
0.13 E-04 \\
0.13 E-04 \\
0.13 E-04 \\
0.13 E-04 \\
0.13 E-04 \\
0.13 E-04\end{array}$ & $\begin{array}{l}\text { REFRCT } \\
\text { ANGLE } \\
89.8 \\
89.8 \\
89.8 \\
89.8 \\
89.8 \\
89.8 \\
89.8 \\
89.8 \\
89.8 \\
89.8 \\
89.8 \\
89.8 \\
89.8 \\
89.8 \\
89.8 \\
89.8 \\
89.8 \\
89.8 \\
89.8 \\
89.8 \\
89.8 \\
89.8 \\
89.8 \\
89.8 \\
89.8 \\
89.8 \\
89.8 \\
89.8 \\
89.8 \\
89.8 \\
89.8 \\
89.8 \\
89.8 \\
89.8 \\
89.8 \\
\end{array}$ & $\begin{array}{l}\text { REFLECTIVITY } \\
0.978486 \\
0.978495 \\
0.978505 \\
0.978516 \\
0.978526 \\
0.978538 \\
0.978550 \\
0.978562 \\
0.978574 \\
0.978588 \\
0.978602 \\
0.978617 \\
0.978633 \\
0.978650 \\
0.978667 \\
0.978686 \\
0.978705 \\
0.978726 \\
0.978747 \\
0.978771 \\
0.978796 \\
0.978822 \\
0.978850 \\
0.978879 \\
0.978913 \\
0.978946 \\
0.978983 \\
0.979023 \\
0.979067 \\
0.979113 \\
0.979164 \\
0.979219 \\
0.979280 \\
0.979346 \\
0.979420 \\
0.9619\end{array}$ \\
\hline
\end{tabular}




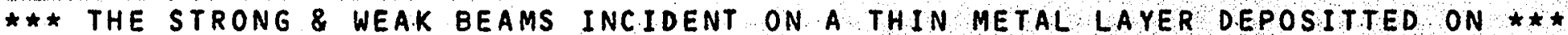
A GLASS-NA GAS INTERFACE AT AN INCIDENT ANGLE OF 50.0 DEGREE

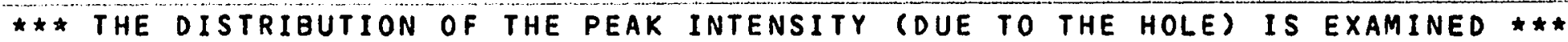
POLARIZATION IS PERPENDICULAR

* * VAN DER WAAL'S POTENTIAL ENERGY HAS THE FORM OF THE INUERSE * * 4TH POWER OF THE NORMAL DISTANCE FROM THE SURFACE

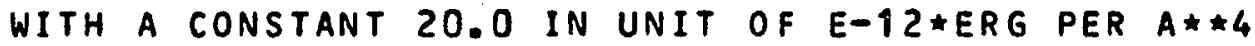

$\star<$ EXPERIMENTAL CONDITIONS >t

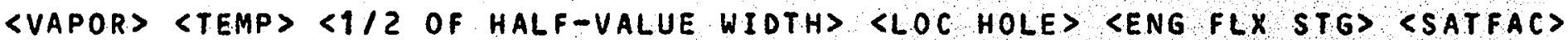
(MMHG) (KELVIN) <HWI> <HWNI> $(\star 10 M H Z) \quad(\star 10 M H Z)$ (WATT/CM**2)
0.067
619.
20.0
4.3
$0.381 \varepsilon-02$
0.60

\begin{tabular}{|c|c|c|c|c|c|c|c|}
\hline $\begin{array}{r}\text { INCIDENT } \\
( \pm 10 M H 2) \\
-180 \\
-176 \\
-172 \\
-168 \\
-164 \\
-160 \\
-156 \\
-152 \\
-148 \\
-144 \\
-140 \\
-136 \\
-132 \\
-128 \\
-124 \\
-120 \\
-116 \\
-112 \\
-108 \\
-104 \\
-100 \\
-96 \\
-92 \\
-88 \\
-84 \\
-80 \\
-76 \\
-72 \\
-68 \\
-64 \\
-60 \\
-56 \\
-52 \\
-48 \\
-44\end{array}$ & 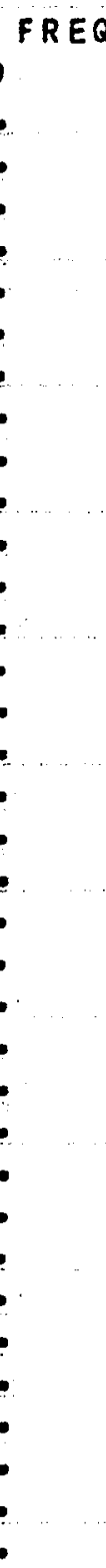 & $\begin{array}{l}\text { DIELECT } \\
\text { REAL } \\
0.999598 \\
0.999598 \\
0.999598 \\
0.999598 \\
0.999598 \\
0.999598 \\
0.999598 \\
0.999598 \\
0.999598 \\
0.999598 \\
0.999598 \\
0.999598 \\
0.999598 \\
0.999598 \\
0.999598 \\
0.999598 \\
0.999598 \\
0.999598 \\
0.999598 \\
0.999598 \\
0.999598 \\
0.999598 \\
0.999598 \\
0.999598 \\
0.999598 \\
0.999598 \\
0.999598 \\
0.999598 \\
0.999598 \\
0.999598 \\
0.999598 \\
0.999598 \\
0.999598 \\
0.999598 \\
0.999598\end{array}$ & $\begin{array}{l}\text { IC CONST } \\
\text { IMAG } \\
0.003725 \\
0.003722 \\
0.003718 \\
0.003715 \\
0.003712 \\
0.003708 \\
0.003705 \\
0.003701 \\
0.003697 \\
0.003693 \\
0.003688 \\
0.003684 \\
0.003679 \\
0.003674 \\
0.003669 \\
0.003664 \\
0.003658 \\
0.003652 \\
0.003645 \\
0.003639 \\
0.003632 \\
0.003624 \\
0.003616 \\
0.003608 \\
0.003599 \\
0.003589 \\
0.003579 \\
0.003568 \\
0.003556 \\
0.003544 \\
0.003530 \\
0.003515 \\
0.003500 \\
0.003482 \\
0.003464\end{array}$ & $\begin{array}{l}\text { REFRACT } \\
\text { INDEX } \\
1.248662 \\
1.248662 \\
1.248662 \\
1.248662 \\
1.248662 \\
1.248662 \\
1.248661 \\
1.248661 \\
1.248661 \\
1.248661 \\
1.248661 \\
1.248661 \\
1.248661 \\
1.248661 \\
1.248661 \\
1.248661 \\
1.248661 \\
1.248661 \\
1.248661 \\
1.248661 \\
1.248661 \\
1.248661 \\
1.248661 \\
1.248661 \\
1.248661 \\
1.248661 \\
1.248661 \\
1.248661 \\
1.248661 \\
1.248661 \\
1.248661 \\
1.248661 \\
1.248660 \\
1.248660 \\
1.248660\end{array}$ & $\begin{array}{r}\text { PENET } \\
\text { DEPTH } \\
0.13 E-04 \\
0.13 E-04 \\
0.13 E-04 \\
0.13 E-04 \\
0.13 E-04 \\
0.13 E-04 \\
0.13 E-04 \\
0.13 E-04 \\
0.13 E-04 \\
0.13 E-04 \\
0.13 E-04 \\
0.13 E-04 \\
0.13 E-04 \\
0.13 E-04 \\
0.13 E-04 \\
0.13 E-04 \\
0.13 E-04 \\
0.13 E-04 \\
0.13 E-04 \\
0.13 E-04 \\
0.13 E-04 \\
0.13 E-04 \\
0.13 E-04 \\
0.13 E-04 \\
0.13 E-04 \\
0.13 E-04 \\
0.13 E-04 \\
0.13 E-04 \\
0.13 E-04 \\
0.13 E-04 \\
0.13 E-04 \\
0.13 E-04 \\
0.13 E-04 \\
0.13 E-04 \\
0.13 E-04\end{array}$ & $\begin{array}{l}\text { REFRCT } \\
\text { ANGLE } \\
89.8 \\
89.8 \\
89.8 \\
89.8 \\
89.8 \\
89.8 \\
89.8 \\
89.8 \\
89.8 \\
89.8 \\
89.8 \\
89.8 \\
89.8 \\
89.8 \\
89.8 \\
89.8 \\
89.8 \\
89.8 \\
89.8 \\
89.8 \\
89.8 \\
89.8 \\
89.8 \\
89.8 \\
89.8 \\
89.8 \\
89.8 \\
89.8 \\
89.8 \\
89.8 \\
89.8 \\
89.8 \\
89.8 \\
89.8 \\
89.8 \\
\end{array}$ & $\begin{array}{l}0.978914 \\
0.978930 \\
0.978948 \\
0.978967 \\
0.9789 .86 \\
0.979006 \\
0.979025 \\
0.979046 \\
0.979069 \\
0.979092 \\
0.979117 \\
0.979141 \\
0.979168 \\
0.979196 \\
0.979225 \\
0.979255 \\
0.979287 \\
0.979321 \\
0.979357 \\
0.979394 \\
0.979434 \\
0.979477 \\
0.979522 \\
0.979569 \\
0.979620 \\
0.979673 \\
0.979729 \\
0.979791 \\
0.979857 \\
0.979927 \\
0.980003 \\
0.980084 \\
0.980174 \\
0.980269 \\
0.980374\end{array}$ \\
\hline
\end{tabular}


\$\$ JOUT, MONI, T

\$: INENT:RFHYAOO I, GAS FLOW

$\$$ :OPTION:FOFITFIAN

\$:FORTRAN

C THE INTENSITY IISSTFIBUTION OF THE WEAK FEFLECTEM BEAM FEOM

C A GLASS-GAS FLOW INTEFFACE

c

C THE GAS FLOW UELOCITY IS ASSUMEI TO BE FAFALLEL TO THE WALL

C THE INSTANTANEOS VELOCITY IS ASSUMEI TO HAUE THE MEAN FI...US

C THE FLUCTUATING FAFT

C A GaUSSIAN FLUCTUATION IS ASSUMEI

C

C

C

C

C

C REWAVE = RESONANCE WAVE LENGTH OF NA I2 LINE

C GASIEN = IIENCITY OF GAS VAFOF (FEF CUEIC CM)

C MOLWEI = MOLCULAF WEIGHT OF ATOM

C HWI =HALF OF $1 /$ E FOINTS WIITH OF INCTIENT BEAM

C HWN1 =HALF OF HALF -UALUE WIITH OF FESONANCE LINE

SOFINI = REFFACTIVE INIEX OF GLASS

091 = DSCTFATOR STLENGTH 1

TEMPER $=$ GAS TEMFEFATUFE

XLIGHT $=$ VELOCITY OF LIGHT

FLANK = FLANK CONSTANT

ELMASS = ELECTOFON MASS

GASCON $=$ GAS CONSTANT

XLOSHM $=$ LDSHMIIIT'S NUMBEF

c AANGLE = ANGLE OF INCIIENCE (IIEGREE)

C ANGINC = ANGLE OF INCIIIECE (FIALIIAN)

C EFIOF $=1 /$ FOINTS WIITH OF IIOFFLEF BFOAIIENEI RESONANCE LINE

C. FAI = FAI

IIMENSION $Z(58)$, FEINI $(58)$, IIEFE $(58)$, IIECM $(58)$, FEF $(58)$,

8. ANGREN(58), IEFT THN (58), FEFLOG (58)

GASTEN $=1$. 05E+15

HWI $=2$.

HWN $1=200$.

TF $1=10$.

TEMFEF $=300$.

FOLAFI $I=+1$

IFROGM $=1$

REWAVE $=5.89 E-5$

XMOLWE $=22+9898$

SATFAC $=0.8$

SORINII $=1,63$

$051=0.42$

$X L$ IGHT $=2.99793 \mathrm{E}+10$

FLANK $=1.05459 \mathrm{E}-27$

ELMASS $=9.10953 E-28$

GASCON=8.31441E+7

BOL TZC $=1$ + 38042E-16 
F'AI $=3.14159$

$X L O S H R=2+68675 E+19$

IIOF $=2+* G A S C O N * T E M F E F / X M O L W E$

EFCLOF $=2+*$ SQRT $($ IIOF $) /$ FEWAUE

$X 10 K H Z=1 \cdot E+4$

$X 1 O M H Z=1, E+7$

EFOOF $=E F C I O F / X 1 O K H Z$

C TO ESTIMATE THE ENERGY FLUX (WATT/CM**2) OF THE STRONG BEAM,

C THE COLISTON FATE IS ASSUMEII TO BE $10 \mathrm{MHZ}$

ENGFLX $=S A T F A C * 137+036 * 2+* X L$ IGHT*ELMASS*2,*HWN1*X10MHZ/FEWAUE/0S1

C

C CONST =CONSTANT AFFERING IN SUSCEFTIEILITY

C.

CONST $1=$ FLANKKGASNEN*REWAVE

CONST $2=137$. 036*ELMASS*SQFT (FAI) *4 * *EFCIOF*FAI **2

CONST $=$ CONST $1 /$ CONST2

WRITE $(6,899)$ CONST

IFOLA $=$ IFIX (FOLARI)

899 FORMAT $(1 H 1, / / /, 3 X, "$ THIS IS A CONSTANT APPERING IN",

$\&$ "SUSCEFTIEILITY", /23X,E12,5)

C

VAFOF=VAFOK FRESSURE IN UNIT OF MMHG

UAFOK $=760 . *$ TEMFEF*GASIEN/XLOSHM $/ 273+2$

$N A=8$

FNA $=F L D A T$ (NA)

$H A=3.2 * H W I / F N A$

CALL FOWEK (AVEFLX, ENGFLX,NA,HA,HWI)

AANGL.E $=40$.

ANGINC=AANGLE $* F A I / 180$.

SOFSIN=SOFI INIKSIN (ANGINC)

CN=CONST $22+/ S O R S I N / T F 1 / S Q F T$ (FAI)

$\mathrm{DLOW}=-0.8 * \mathrm{TF} 1$

IIHIG $=0.8 *$ TFI

FNDII $=0+8 * I H I G / 0+1$

NIII $=$ TFIX (FNIII)

FNII $=2$, *FLOAT (NOII)

$N I=$ IF IX (FNII)

$\mathrm{HII}=2$ + *IHIG/FNI

TUL $=\times 10 K H Z *$ KEWAUE*TF1/SORSIN

TKE $=$ TVL $* * 2$

I $V A N=3$

$\mathrm{ZERO}=0$.

TIO 4600 IUANRE=1, IUAN

INTFAC = TUANLE-3

$F \cup M=0$.

IF (INTRAC, EQ, - 2)FUM $=+3$,

IF ( INTRAC, $E R,-1) F \cup M=+12$,

UM $=X 10 K H Z * F E W A U E * F U M / S O F S T N$

IFREQ $=18$

$F S C=2$.

FIFREQ =FLOAT (IFREQ) 
FATIO=FIFREQ $/ F S C+0+5$

RATIO2 $=2$ + *FATIO

IC $=$ IF IX (FATIO2)

IFFEQ $=$ IFFEQ-IFIX (FUM)

IF (INTFAC + LE + -1)F IFKEQ =FIFGEQ -FUM

Iio 4000 IZ $Z=1$, IC

$I I Z=I Z-1$

$Z(I Z)=-F I F R E Q+F L O A T(I I Z) * F S C$

IHOLE $=0$

CALL SUSCEF (FESUM, CMSUM, Z(IZ), NI, HII,

\& OLOW, CN, OS1, HWN1, IHOLE,

\& SATFAC, FUM, TFI,HWI)

FESUMII=RESUM

CMSUMI $=$ CMSUM

IHOLE $=1$

CALL SUSCEF (FESUM, CMSUM, $Z(I Z)$, NII YHII,

\& OLOW, CN,OS1,HWNL, IHOLE,

SATFAC, FUM, TFI, HWI)

FESUMH $=$ FEESUM

CMSUMH $=$ CMSUM

RESUM1 =FESUMII-FESUMH

CMSUM $=$ CMSUMII-CMSUMH

4130 CALL FEFINI (FEINIX, FEFFEX , FEFCMX, ALFAX,

\& BETAX, RESUMI, CMSUMI, SOFINI, ANG INC, FAI)

REIND $(I Z)=$ FEINIX

IIEFE $(I Z)=$ REFREX

IIIECM $(I Z)=$ REFCMX

ALFAN=AL.FAX

BETAN=EETAX

IIEPTHN $(\mathrm{IZ})=$ FEWAUE/BETAN/FAI $/ 2$.

ANGLEZ $=50 F S I N / F E I N I(I Z)$

ANGFEN $(I Z)=180$, *AFSIN (ANGLE2) /FAI

CALL FEFLEC \ALFEFLFX, FEFLFX, ALFAN, BETAN, ANGINC , ANGFEF,

$\&$ POLALI , SOFINII)

REF $(I Z)=.F E F L F X$

4000 CONTINUE

FEFLOG $(I Z)=A L F E F L F X$

WFITE $(6,901)$ AANGLE

IF (IFOLA.LT, O) WFITE $(6,898)$

IF ( IFOLA, GT , O)WFITE $(6,897)$

IF (IFOLA,EQ.0)WFITE $(6,896)$

WRITE $(6,840)$

WRITE $(6,850)$ UM, TKE, TVL.

WFI IE (6, 908 ) VAFOF, TEMFEF , HWI, HWN1, FUM,

AUEFLX, SATFAC

WFITE $(6,909)$

WFITE $(6,910)$ (Z(IZ), IIEFE(IZ), IIECM(IZ), FEINI (IZ), IIEPTHN(IZ),

\& ANGREN (IZ), REF $(I Z), I Z=1, I C)$

WF ITE $(6,955)$

CALL GRAFH (FUM, UM, TF1, TUL, IC, IFREQ, FSC, ITEFE)

WFITE $(6,956)$

CALL GFAFH (FUM, UM, TFI, TUL, IC, IFFER,FSC, IIIECM)

WFITE (6.958)

CALL GRAFH (FUM, UM, TFI, TVL, IC, IFREQ,FSC, REF) 
WRITE $(6,960)$

CALL GFAPH (FUM,UM, TF1, TUL, IC, IFREQ,FSC, REFLOG)

4600 CONTINUE

898 FOFIMAT (1HO,23X, "POLAFIZATION IS FAFALLEL")

897 FOFMAT 1 1HO, $23 \times$, FOLAFIZATION IS FEFFENIICULAR")

996 FORMAT (1H0,23X, "FLANE FOLAFIZEI LIGHT AT 45 IEGREE")

908 FOFMAT $(140,17 X$, * EXFEFIMENTAL CONDITIONS $\% * * \%$

\& IHO, $2 X, \angle U A F O R$ OTEMF $\angle 1 / 2$ OF HALF-UALUE WIOTH 2 ,

\& "LOC HOLE\% CENG FLX STG\% \%SATFAC\%", $/ 3 X$ " (MMHG)"

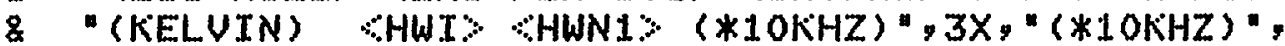

\& $3 X_{*}$ "(WATT/CM**2)" $/ 1$ HO

\& $3 X, F 5+3,4 X, F 4,0,4 X, F 4,1,2 X, F G, 1,10 X$,

8. $3 X, F 6,1,6 X, E 10,3,5 X, F 3,1,1)$

901 FORMAT $1 H 1 \% / / / 3 X, " * * *$ A NARROW FRORING BEAM INCILENT ON",

\& A GLASS-NA GAS FLOW INTEFIFACE ***",/2OX, "AT AN" "

\& "INCIUENT ANGLE OF " "FA,1," IIEGREE",/)

906 FORMAT 1 1HO, 13X, "LINE SHAFE OF THE HOLE IS EXAMINEN" " $)$

910 FDRMAT (1H, 4X,F5, 0, 4X,F8,6,1X,F8,6,1X,F8,6,1X,E8,2, 2X,FA, I,

\& $\quad 4 X, F 8+6)$

909 FORMAT 1 HO, "INCIIENT FFEQ" $1 \mathrm{x}$, IIELECTRIC CONST" , 2X, "FEFRACT",

\& $4 X$, "FENET" "2X, "FEFFCT" "2X, "FEFLECTIUITY" , $2 X, "(* 1 O K H Z) "$ "

\& 6X, "REAL" , 4X, "IMAG", 5X, "INDEX" , 6X, "IEFTH" , 3X, "ANGLE" ,/)

955 FORMAT $(1 H 1, / /, "$ FFER $\%, 34 X$ " $F$ FEL FART OF THE IIELECTFIC", $\& \quad$ CONSTY" $\%$

956 FORMAT $(1 H 1, / / 1, " \angle F R E R \%, 34 \times, " \triangle I M A G$ FART OF THE IIELECTRIC", $8 \quad$ CONST:",1)

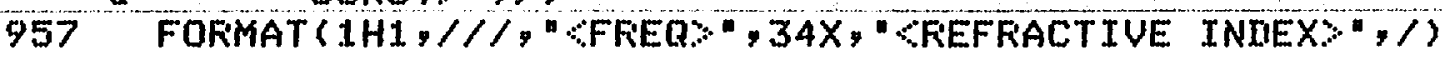

958 FOFMAT $\{1 H 1,1 /, "$ FFER\%" ,34X, "FEFLECTIUTTY\%",

840 FOFMAT (1HO, 25X, "***GAS FLOW CONITIONS ***")

960 FORMAT $(1 H 1, / / /$ " $\angle F F E Q \%, 20 X$ " NATUFAL LOG OF THE REFLECTIUTY ")

850 FORMAT $(1 H 0,19 X, "<$ MEAN FLOW UELOCITY=",F7,2, "(CM/SEC) $\% " /$

$\& \quad 20 X, " \angle$ TUREULENCE KINETIC ENGY $=", F 7.2, "(C N / S E C) * * 2 \% ", 1$

\& $2 O X, " \because$ INTENCITY OF TUFBULENCE $=" F 7+2, "(C M / S E C) \geqslant ")$

9000 STOF

ENII

C SURROUTION TO GRAFH IIATA

SUEROUTINE GRAFH (FUM, UM, TF1, TUL, TC , TFREQ, FSC, UALUE)

IITMENSION FLOT (B3), VALUE (IC)

IIATA IIASH/"-"/,BAR/"I"/,X/"*"/,ELANK/" "/

IO $5 \quad I=1$, IC

IF (I.EQ , I) YMAX $=$ UALUE (I)

IF (I.EQ + I) YMIN=UALUE(I)

IF (I.GE, 2) GO TO 2

GO TO 5

2 BIG $=$ VALUE (I) -YMAX

SMALL = VALUE (I ) -YMIN

$Z E R O=0$.

IF (AIG,GE,ZERO) YMAX=UALUE (I)

IF (SMALL.LT +ZERO) YMIN=VALUE (I)

5 CONT INUE

IIIF $=$ YMAX $-Y M I N$

IIO $10 \quad K=1,83$

FLOT $(K)=$ IIASH 
10 CONTINUE

WFITE $(6,20)$ FLOT

20 FORMAT $(1 X, "(* 10 K H Z) ", / 1 H 0,17 X, 83 A 1)$

no $30 \quad K=1,83$

FLOT $(K)=$ BLANK

30 CONTINUE

$\operatorname{PLOT}(1)=\mathrm{BAF}$

no $60 \quad K Z=1, I C$

$K Z Z=K Z-1$

$Z=-F L O A T(I F R E R)+F L D A T(K Z Z) * F S C$

YIIF =UALUE (KZ) -.YMIN

$Y N=Y I I F * 80, / D I F+2+5$

$N Y=I F I X(Y N)$

FLOT $(N Y)=X$

WFITE $(6,40) Z$, VALUE (KZ), FLOT

40 FOFMAT $(1 X, F 5,0,2 X, F B, 6,1 X, 83 \mathrm{~A} 1)$

FLOT $(N Y)=E L A N K$

60 CONTINUE

WRITE $(6,61)$

WRITE $(6,62)$ FUM, UM, TFI, TUL

62 FOFMAT (1HO, "* THE FREQ SHIFT",F7+2," (*1OKHZ) COFESFONDS TO THE "

\& "MEAN FLOW VELOCITY",F7+2y" (CM/SEC) * " $/ 1 X, *$ THE 1/E ",

\& "FOINTS" WIITH" F7.2," (*1OKHZ) COFESFONIS TO THE FLUCTUATIO",

\& OF"F7.2, (CM/SEC) * $*$ )

61 FOFMAT 1 1H0,20X, ***** THE CENTRAL FFEQ OF THE UNSHIFTEI "

\& "NATURAL LINE IS LOCATEI AT FREQ $=0$ + *****)

RETUKIN

ENI

SURFOUTINE FOWEF (AUEFLX,ENGFLX,N,H,HWT)

SFAA $=0$.

IIO $10 \mathrm{~J}=1, N+1,2$

$J L=\sqrt{ }-1$

$\mathrm{II}=\mathrm{H} * \mathrm{FLOAT}(\mathrm{JL})-1.6 * \mathrm{HWI}$

$A 1=-I 1 * 2 / H W I * 2$

$E A 1=E X F(A I)$

SRAA $=$ SRAATEAI

10 CONTINUE

AUEFL $X=E N G F L X * S F A A /(F L O A T(N)+1)$

FETURN

ENII

SURFOUT INE SUSCEFCFESUM, CMSUM, $Z, N, H$,

\& XLOWAL, C, OS1, HWNI,

\& IHOLE, SATFAC, FUM, TFI, HWI)

$Z=Z-F U M$

$5 \quad$ SREAB $=0$.

SCMAE $=0$.

IIO $16 \quad L A=1, N+1,2$

$L L A=L A-1$

DAL $=H * F L O A T(L L A)+X L O W A L$

SFRAE $=0$.

SCFIAE $=0$.

IIO 17 II $=1,2$

$N N=I I-1$

FNN $=F L O A T(N N)$ 
U1 $=\mathrm{I} A L+H * F N N$

$F U N=-4+* U_{1} * * 2 / T F 1 * * 2$

EAL $1=E X F$ ( $F U N$ )

ZHOLE $=V_{1}$

$A L L 2=(Z-Z H O L E) * * 2 / H W I * * 2$

IF (ALL2, GT.60)G0 TO 90

$E A L 2=E X P(-A L L 2)$

SATFA1 $=$ SATFAC *EAL?

GO TO 100

$90 \quad$ SATFAI $=0$.

$100 \quad$ ALZI $=-Z-U 1$

AL $Z 2=A L Z 1 * 2$

IF (IHOLE, EQ, 1 ) ALZF' $1=0$.

IF (IHOLE,EQ + 1) ALZF' $=A L Z F 1 * * 2$

AL ZZZ =ALZ1**2

HWN2 $=H W N 1 * * 2$

$A L X 1=A L Z 2+H W N 2$

SATFA2 $=1$, +SATFA1

IF (IHOLE , EQ . I ) HOLE=SATFAI*HWN2/2,/(ALZF2+HWN2*SATFA2)

FFAL $1=E A L 1 * 0 S 1 * A L Z 1 / A L X 1$

CFAL $1=E A L 1 * H W N 1 * 0 S 1 /$ AL X1

IF $($ IHOLE, EQ + 1 ) FIFAL $1=$ HOLE *FFFAL 1

IF (IHOLE,$E Q+1)$ CFAL $1=H O L E * C F A L 1$

IF (LLA,EQ, O)GO TO 12

IF (LLA,EQ,N)GQ TO 13

IF $(N N, E R+O) N C=2$

$I F(N N, E Q, \perp) N C=4$

GO TO 14

12 IF $(N N \cdot E Q+O) N C=1$

$I F(N N+E Q, 1) N C=4$

GO TO 14

13 IF $(N N, E Q, 0) N C=1$

$I F(N N \cdot E Q+I) N C=0$

$1.4 \quad F N C=F L D A T$ (NC)

SFFAE = SFFAE+FNC *FIFAL 1

SCFAE = SCKAE +FNC *CFAL 1

17 CONTINUE

SREAE=SRRAE+SREAB

SCMAE = SCMAE+SCFAE

16 CONTINUE

FUNREY $=H * S R E A R / 3$.

FUNCNY $=H * S C M A E / 3$.

31 FESUM=C*FUNFEY

CMSUM $=$ C*FUNCMY

$Z=Z+F \cup M$

32 FETUFN

ENI

C. SUEROUTINE TO CALCULATE FEFRACT TUE INIEX

SUEROUTINE FEFINI (REINIX, FEFREX, FEFCMX, ALFAX, BETAX,

\& SUSFEX, SUSCMX, SOINIX, ANGINC, FAI)

REFFEX $=1++4$ * *F'AI*SUSFEX

REFCMX $=2$. *PAI $*$ SUSCMX

FAFT $1=($ SOINAX $* S I N(A N G I N C)) * * 2$ 
$A=F E F F E X-F A R T 1$

$\mathrm{A}=2$. *KEFCHX

C=REFFEX+F'AFIT 1

$n=0$.

$A E=A B S(A)$

IF $(A B-B) 60,61,62$

$60 \quad X=A / B$

$X F T=1+X * * 2$

ALFA $=E *(S R F T(X F T)+X) / 2$.

ALFAX $=S$ SRT ( $A L F A$ )

BETA $=\mathrm{B} *(\operatorname{SRFT}(X F T)-X) / 2$.

$E=S Q R T$ (XRT) $-X$

IF (E. EQ , II) $B E T A X=0$.

EETAX $=$ SQF T ( $B E T A)$

FAFIT $X=(C+E * X F T) / 2$.

FEINIX $=$ SRFT (PARTX)

G0 TO 63

61 ALFA $=\mathrm{E} *(1,+5 \mathrm{SFT}(2)) /$,2 .

ALFAX $=S Q R F T$ (ALFA)

BETA $=B *(\operatorname{SRRT}(2)-1) /$,2 .

BETAX $=$ SRFT ( $R E T A$ )

FARTE $=(C+B *(1+\operatorname{SRFT}(2))) /$,2 ,

FEINIX $=S Q R$ ST (FARTE)

GO TO 63

62. $Y=E / A$

$Y F T=1+Y * * 2$

$A L F A=A *(1,+S Q F T(Y F T)) / 2$.

IF $(A+L T+I)$ ALFA=AE* $(S Q R T(Y F T)-1) /$,2 .

ALFAX $=$ SQRT (ALFA)

BETA $=A *(S Q R T(Y R T)-1) /$,2 .

IF (A,LT, D) EETA=AE* (SRFT $(Y F T)+1,) / 2$.

$F=S$ SQRT (YRT) -1 .

$I F(F+E Q+I)$ EETAX $=0$.

EETAX $=S R F T$ ( $\mathrm{EETA}$ )

F.AFTY $=(C+A * Y R T) / 2$.

FEINIX $=S$ SRFT (FARTY)

63 IF (FEINIXX*2-FAFT1) 64,65,65

$64 \quad X C 1=$ FEFCMX**2/(FART1-FEINIX**2)

$X C 2=X C 1+F A F T 1$

$X C=S Q F T(X C 2)$

FEINIX $=X C$

65 FETURN

ENI

C SUEFOUTINE TO IIEFINE A FUNCTIONAL FOFM OF FEFLECTIUITY

SUEROUTINE REFLEC (ALFEFLFX, REFLFX, ALFAX, RETAX, ANGINC,

\& ANGREF, FOLARII, SOINIXX)

$A C=\operatorname{COS}$ ( ANGINC)

$A S=S I N$ (ANGINC)

FINUMAX $=(5 O$ INIIX*AC-ALFAX) $* * 2+$ EETAX**2

RIIENO=(SOINIX*AC +ALFAX) **2+BETAX**2

ALFNUMAX $=A L O G$ (FNUMAX)

ALFIENO=ALOG (RIENO) 


\section{$S A=S O I N D X * A S$}

$X A=(A L F A X * * 2-$ EETAX**2+SA**2)*AC

XAN $=X A-S O I N I X * A L F A X$

$X B=2$ * *ALFAX $* B E T A X$

$X E N=X E-S O I N I X *$ RETAX

FNPER $=X A N * * 2+X E N * * 2$

$X A D=X A+S O I N I X * A L F A X$

$X E D=X B+$ SOINIIX $*$ FETAX

RIPER $=$ XAII* $* 2+X$ EIN* $* 2$

ALRNFEF $=A L O G$ ( $R$ NFFEF')

ALRUIFE $=A L O G$ ( $R I F E R$ )

IF (FOLARI) 701,702,703

701 REFLFX=FNFER/RIFER

ALREFLFX $=A L F N F E R-A L R D F E R$

GO TO 705

703 REFLFX $=$ RNUMAX/RDENO

ALREFLFX $=A L R N U M A X$-ALRIIENO

60 TO 705

702 REFLFX $=.5 *$ (RNFEF/RIFERTFIUMAX/RIENO)

ALREFLFX $=0.5 *$ (ALFNFER+ALLNUMAX-ALFIFER-ALFIIEND)

705 RETURN

ENR

\$.EXECUTE

\$LIMITS: $10,27 K, 5000$

\$: SYSOUT:06, ORG

Q TENTIJOE 


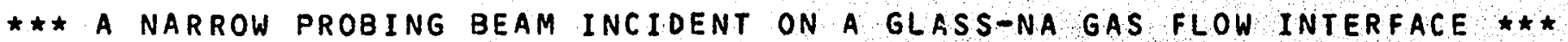
AT AN INCIDENT ANGLE OF 40.0 DEGREE

POLARIZATION IS PERPENDICULAR

$\star \star \star$ G.A.S FLOW COND.TIONS_kt*

< MEAN FLOW VELOCITY $=$ O. (CM/SEC) >

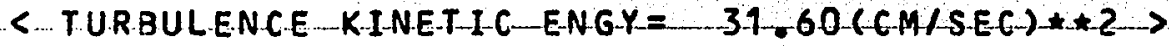

< INTENCITY OF TURBULENCE $=5.62(\mathrm{M} / \mathrm{SEC})>$

$\star<$ EXPERIMENTAL CONDITIIONS $>*$

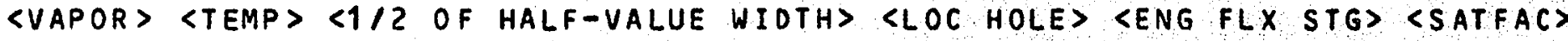
(MMHG)

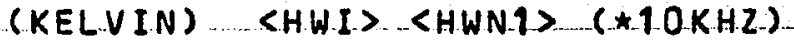
( $\star 1.0 K+2$. (WATTIACM*A-2)
0.033
300 .
$2.0 \quad 200.0$
0.
$0.238 E 00$
0.8

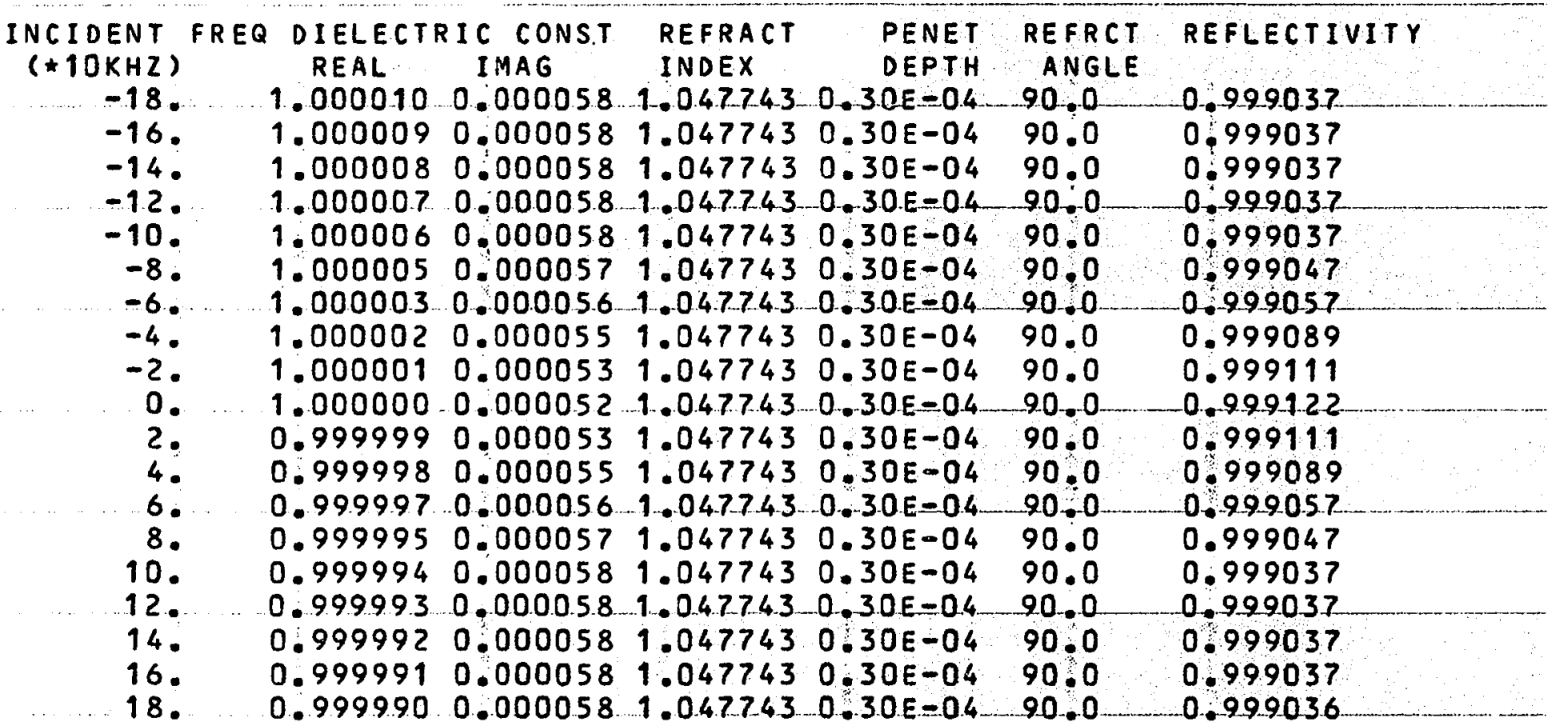


$-18.0 .999037 \quad$ I*

$-16 . \quad 0.9 .99037$ I*

$-14=0.999037$ I*

-12. 0.999037 I*

$-10 . \quad 0.99 .9037 \ldots$

-8. 0.9990471

-6.0 .999057 I

$-4 . \quad 0.9990891$

-2. $\quad 0.999111$ I

0. 0.999122 I

2. 0.999111 I

4. 0.999089 I

6. 0.999057 I

8. 0.99904 .7 I

10. 0.999037 I*

12. 0.9 .99037 I*

14.0 .999037 I*

16. 0.99 .9037 I*

18. $0.999036 I$ I*

* * * THE CENTRAL FREQ OF THE UNSHIFTED NATURAL LINE IS LOCATED AT FREQ=0.

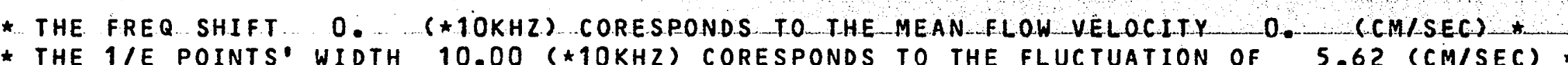


182

* * A NARROW PROBING BEAM INCIDENT ON A GLASS-NA GAS FLOW INTERFACE *** AT AN INCIDENT ANGLE OF 40.0 DEGREE

POLARIZATION IS PERPENDICULAR

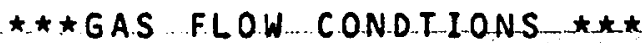

< MEAN FLOW VELOCITY $=1.69$ (CM/SEC) >

<. TURBULENCE PINETIC-ENGY $=31-60(\mathrm{CM} / \mathrm{SEC}) * 2+2>$

< INTENCITY OF TURBULENCE $=5.62(\mathrm{CM} / \mathrm{SEC})$ >

* < EXPERIMENTAL CONDITIONS $>*$

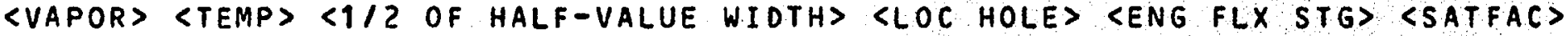
(MM.HG.) (KELVIN) .... <HWI. SHWN.1> (*1.OKHZ) $(\star 1.0 K \cdot H 2)$ (WATT/CM*t2)
0.033
300.
$2.0 \quad 200.0$
3.0
$0.238 E \quad 00$
0.8

\begin{tabular}{|c|c|c|c|c|c|c|}
\hline 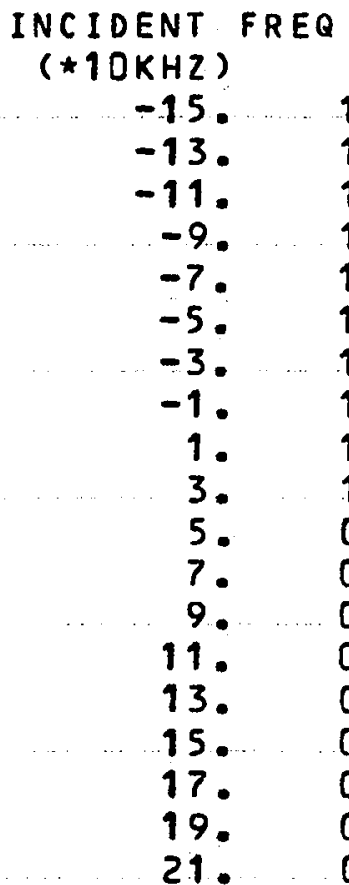 & $\begin{array}{l}\text { DIELECT } \\
\text { REAL } \\
1.000010 \\
1.000009 \\
1.000008 \\
1.000007 \\
1.000006 \\
1.000005 \\
1.000003 \\
1.000002 \\
1.000001 \\
1.000000 \\
0.999999 \\
0.999998 \\
0.999997 \\
0.999995 \\
0.999994 \\
0.999 .993 \\
0.999992 \\
0.999991 \\
0.9999 .90\end{array}$ & $\begin{array}{l}\text { RIC CONST } \\
\text { IMAG } \\
0.000058 \\
0.000058 \\
0.000058 \\
0.000058 \\
0.000058 \\
0.000057 \\
0.0000 .56 \\
0.000055 \\
0.000053 \\
0.000052 \\
0.000053 \\
0.000055 \\
0.000056 \\
0.000057 \\
0.000058 \\
0.000058 \\
0.000058 \\
0.000058 \\
0.000058\end{array}$ & $\begin{array}{l}\text { REFRACT } \\
\text { INDEX } \\
1.0 .477 .43 \\
1.047743 \\
1.047743 \\
1.0477 .43 \\
1.047743 \\
1.047743 \\
1.0477 .43 \\
1.047743 \\
1.047743 \\
1.0477 .43 \\
1.047743 \\
1.047743 \\
1.047743 \\
1.047743 \\
1.047743 \\
1.047743 \\
1.047743 \\
1.047743 \\
1.047743\end{array}$ & $\begin{array}{r}\text { PENET } \\
\text { DEPTH } \\
0.30 E-0.4 \\
0.30 E-04 \\
0.30 E-04 \\
0.30 E-0.4 \\
0.30 E-0.4 \\
0.30 E-04 \\
0.30 E-0.4 \\
0.30 E-04 \\
0.30 E-04 \\
0.30 E-0.4 \\
0.30 E-04 \\
0.30 E-04 \\
0.30 E-0.4 \\
0.30 E-04 \\
0.30 E-04 \\
0.30 E-0.4 \\
0.30 E-0.4 \\
0.30 E-04 \\
0.30 E-0.4\end{array}$ & $\begin{array}{l}\text { REFRCT } \\
\text { ANGLE } \\
90.0 \\
90.0 \\
90.0 \\
90.0 \\
90.0 \\
90.0 \\
90.0 \\
90.0 \\
90.0 \\
90.0 \\
90.0 \\
90.0 \\
90.0 \\
90.0 \\
90.0 \\
90.0 \\
90.0 \\
90.0 \\
90.0\end{array}$ & $\begin{array}{l}\text { REFLECTIVITY } \\
0.999037 \\
0.999037 \\
0.999037 \\
0.999037 \\
0.999037 \\
0.999047 \\
0.999057 \\
0.999089 \\
0.999111 \\
0.999122 \\
0.999111 \\
0.999089 \\
0.999057 \\
0.999047 \\
0.999037 \\
0.999037 \\
0.999037 \\
0.999037 \\
0.999036\end{array}$ \\
\hline
\end{tabular}


-15. 0.999037 I*

$-13.0 .99 .9037 \ldots$. $I *$

-11. 0.999037 I*

-9.0 .999037 I*

-7. $\quad 0.999037$ I.

-5 . 0.999047 I

-3. 0.999057

$-1 . \quad 0.99 .9089$ I

1. 0.9991111

3. 0.99 .9122 I

5. $\quad 0.999111$ I

7. 0.999089 I

9. 0.999057 I

11. 0.999047 I

13. 0.999037 I*

15. 0.999037 I*

17. 0.9290371 1*

19. $0.999037 \mathrm{I}$ *

21. 0.999036 I*

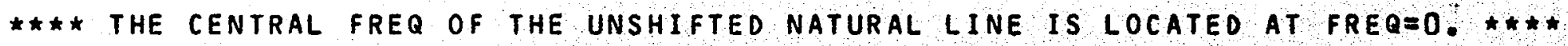

* THE FREQ SHIFT 3.00 (*10KHZ) CORESPONDS TO THE MEAN ELOW VELOCITY 1.69 (CM/SEC) *

* THE $1 /$ E POINTS' WIDTH 10.00 ( $\$ 10 K H Z)$ CORESPONDS TO THE FLUCTUATION OF 5.62 (CM/SEC) * 